\title{
Checklist of British and Irish Hymenoptera - Braconidae
}

\author{
Gavin R. Broad ${ }^{\ddagger}$, Mark R. Shaw§, H. Charles J. Godfrayl \\ ‡ The Natural History Museum, London, United Kingdom \\ $\S$ National Museums of Scotland, Edinburgh, United Kingdom \\ | University of Oxford, Oxford, United Kingdom
}

Corresponding author: Gavin R. Broad (g.broad@nhm.ac.uk)

Academic editor: Pavel Stoev

Received: 17 Feb 2016 | Accepted: 11 Apr 2016 | Published: 21 Apr 2016

Citation: Broad G, Shaw M, Godfray H (2016) Checklist of British and Irish Hymenoptera - Braconidae.

Biodiversity Data Journal 4: e8151. doi: 10.3897/BDJ.4.e8151

\section{Abstract}

\section{Background}

The checklist of British and Irish Braconidae is revised, based in large part on the collections of the National Museums of Scotland, Edinburgh, and the Natural History Museum, London. Distribution records are provided at the country level together with extensive synonymy and bibliography.

\section{New information}

Of the 1,338 species regarded as valid, presumed native and certainly identified, 83 are here recorded for the first time from the British Isles. One new synonym is established (Dyscritus suffolciensis Morley, 1933 = Syntretus splendidus (Marshall, 1887) syn. nov.)

\section{Keywords}

Britain, Ireland, fauna, Ichneumonoidea 


\section{Introduction}

The Braconidae is one of two families of the superfamily Ichneumonoidea, along with the Ichneumonidae. Given the size of each family in Britain (over 1,300 braconids and 2,500 ichneumonids) we are publishing the two checklists separately. This is one part of a series of papers revising the British and Irish list of Hymenoptera, that started with Broad and Livermore (2014a), Broad and Livermore (2014b) and Liston et al. (2014). For the background and rationale behind these British Hymenoptera checklists see Broad (2014). The bases for the taxonomy are Fauna Europaea and Taxapad (Yu et al. 2012) (braconid data for both compiled by Kees van Achterberg) but we have not relied on these sources for data on species occurrence in Britain. Rather, we have relied mostly on the primary literature and collections, especially NMS and BMNH. Indeed, large parts of the checklist rely on original work, identifying specimens in these collections.

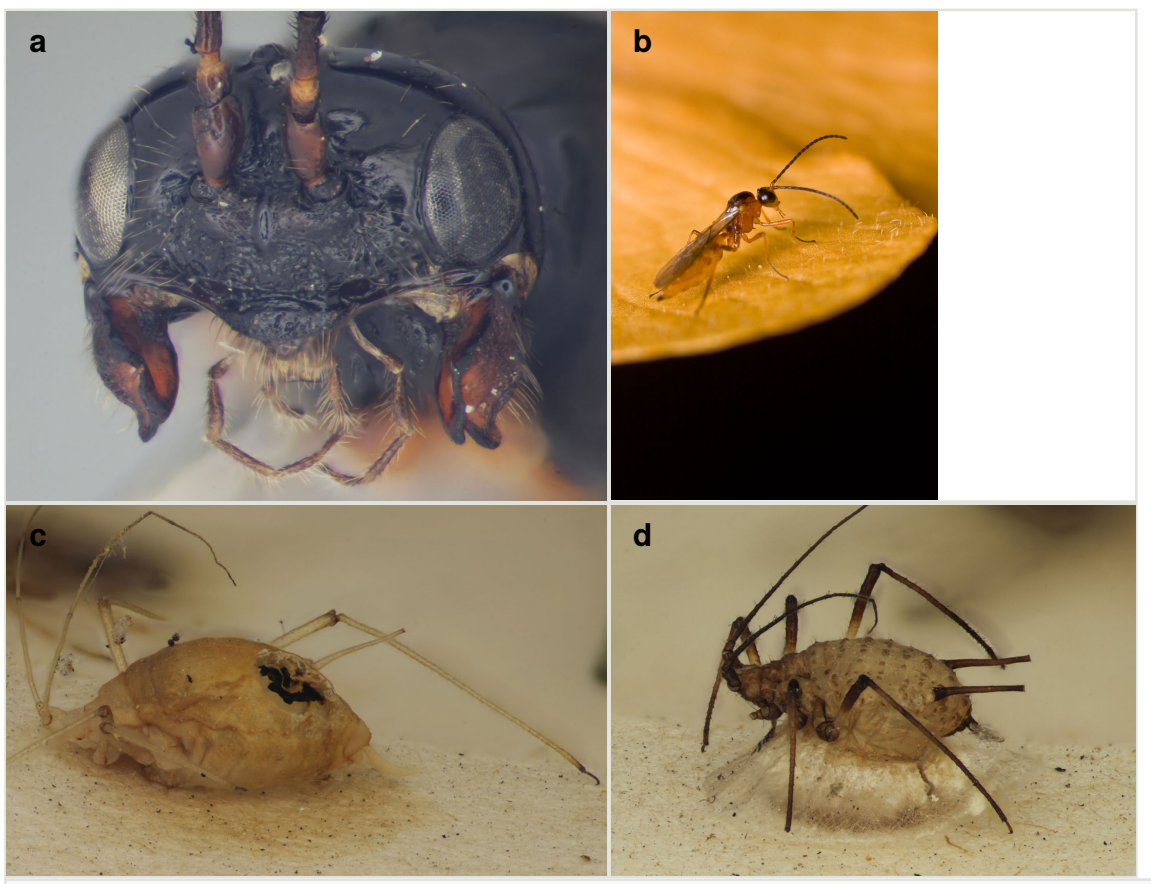

Figure 1.

Representative British Braconidae: Alysiinae and Aphidiinae

a: Face of Alsysia manducator (Panzer) (Alysiinae), with exodont mandibles for emerging from Diptera puparia. BMNH specimen.

b: Pauesia sp. (Aphidiinae) (courtesy of P. Adams)

c: Aphidius ervi (Haliday) (Aphidiinae): mummified aphid host that the braconid pupates within. BMNH specimen.

d: Praon volucre (Haliday) (Aphidiinae): mummified aphid host with the braconid pupa beneath. BMNH specimen. 
Much of the synonymy adopted here is equivalent to the German list (Belokobylskij et al. 2003) but where there are taxonomic differences of opinion, usually van Achterberg's interpretation has been followed, largely for consistency with Fauna Europaea, but also because of van Achterberg's experience with many groups of braconids. The biggest departure from these sources is in the generic classification of Microgastrinae, for which we largely follow Mason (1981).

We reference all additions to and deletions from the British list since Huddleston (1978) and record country-level distribution within the British Isles, but regarding Ireland as one. We made much use of O'Connor et al. (1999) for Irish records. Because the braconid literature is extensive and scattered we also provide many taxonomic references. The braconid section of the 1978 British checklist (Huddleston 1978) repeats many of Shenefelt's (e.g. Shenefelt 1973, Shenefelt 1974) mistakes and these are rectified here.

Figs 1, 2, 3, 4 illustrate a tiny part of the morphological and biological diversity of Braconidae. Shaw and Huddleston (1991) provide an introduction to the varied biology of this family of parasitoid wasps.

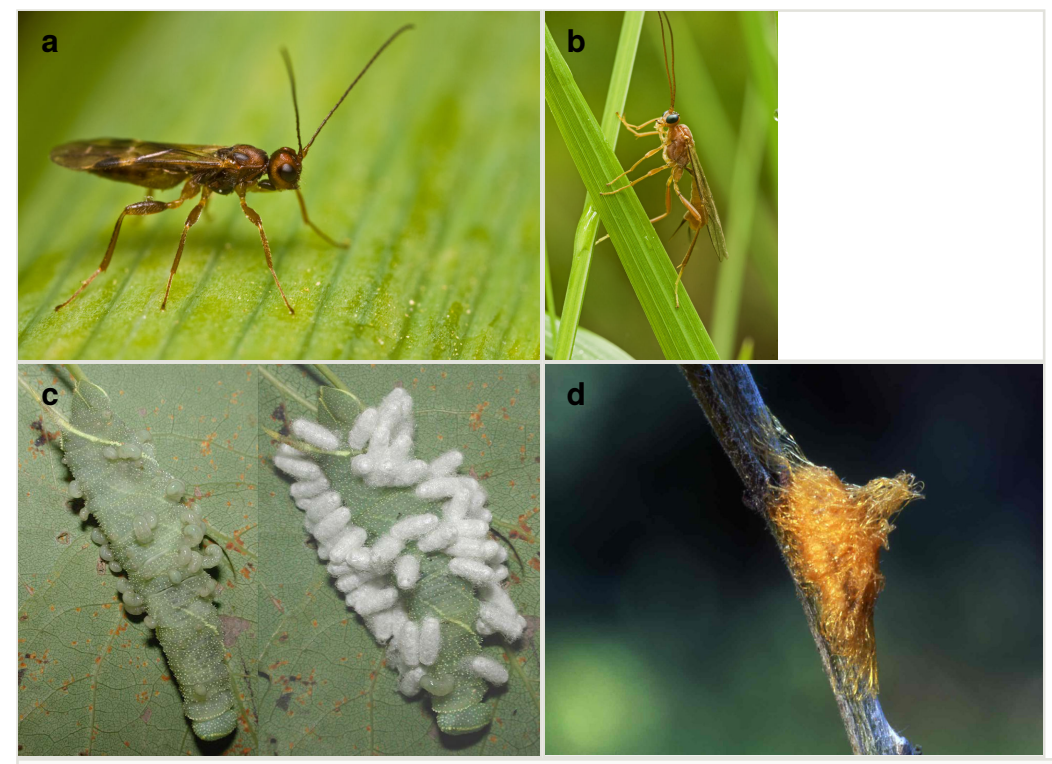

Figure 2.

Representative British Braconidae: Doryctinae, Meteorinae and Microgastrinae

a: Dendrosoter protuberans (Nees) (Doryctinae) male (courtesy of P. Adams)

b: Zele albiditarsus Curtis (Meteorinae) female (courtesy of E. Brosens)

c: Cotesia affinis (Nees) (Microgastrinae) larvae emerging from their Laothoe populi (L.) (Lepidoptera: Sphingidae) host and then spinning cocoons (courtesy of M. Boddington)

d: Cotesia gonopterygis (Marshall) (Microgastrinae) overwintering cocoon mass ex Gonepteryx rhamni (L.) (Lepidoptera: Pieridae) (M.R. Shaw) 


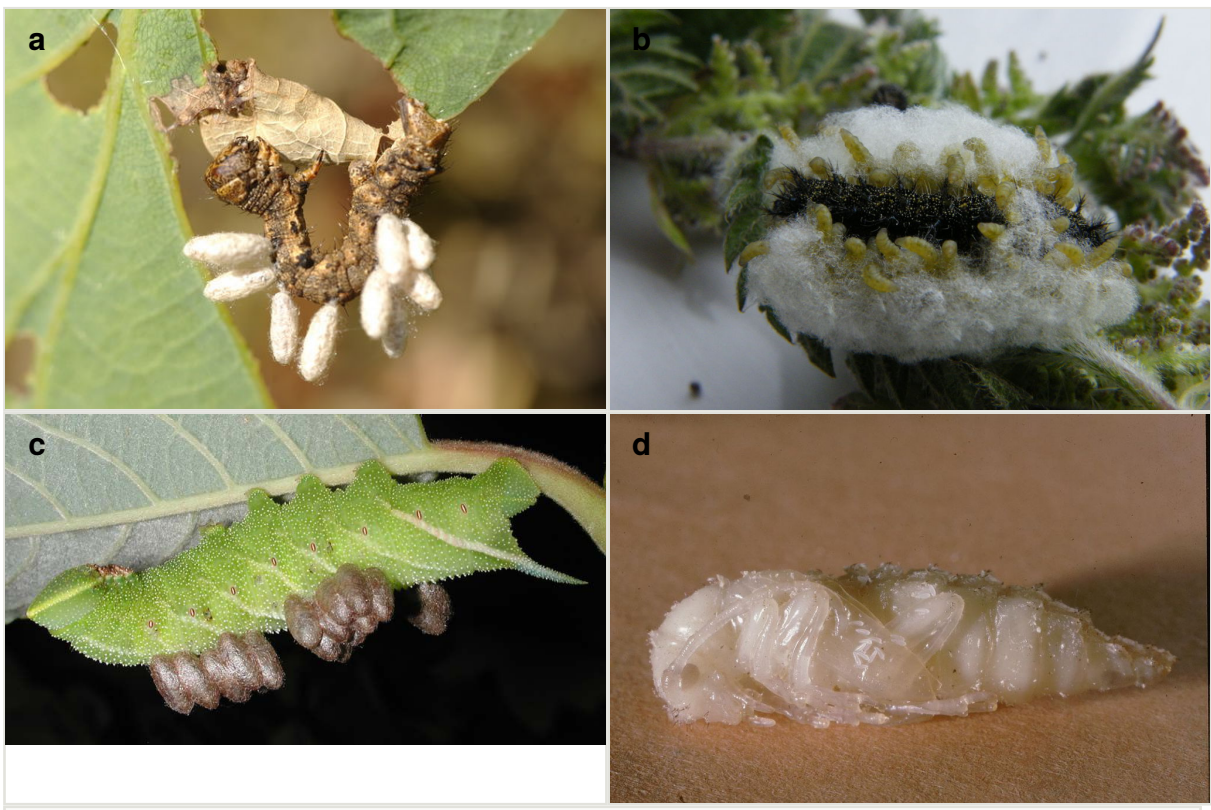

Figure 3.

Representative British Braconidae

a: Cotesia spuria (Wesmael) (Microgastrinae) cocoons ex larval host Apocheima hispidaria (D. \& S.) (Lepidoptera: Geometridae) (courtesy of J. Voogd)

b: Cotesia vanessae (Reinhard) (Microgastrinae) emerging from larval host Aglais urticae (Linnaeus) (Lepidoptera: Nymphalidae) (courtesy of N. Spring)

c: Microplitis ocellatae (Bouché) (Microgastrinae) cocoons ex larval host Smerinthus ocellatus (Linnaeus) (Lepidoptera: Sphingidae) (courtesy of M. Boddington)

d: Histeromerus mystacinus Wesmael (Rhyssalinae) eggs on pupal host Stictoleptura scutellata (L.) (Coleoptera: Cerambycidae) (M.R. Shaw)

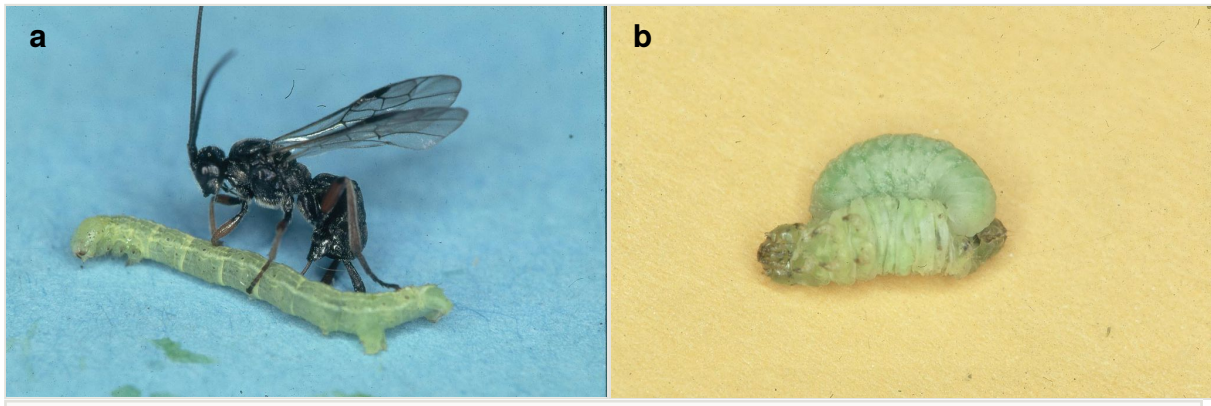

Figure 4.

Representative British Braconidae: Sigalphinae

a: Acampsis alternipes (Nees) ovipositing in larval host, Alsophila aescularia (D. \& S.) (Lepidoptera: Geometridae) (M.R. Shaw)

b: Acampsis alternipes (Nees) larva emerging from its larval host Alsophila aescularia (D. \&

S.) (Lepidoptera: Geometridae) to complete its feeding externally (M.R. Shaw) 


\section{Materials and methods}

For a more detailed description of the background and rationale to the Hymenoptera checklist, see Broad (2014). We have tried to account for every name on the 1978 (Huddleston 1978) checklist and have referenced all additions to and deletions from that list. We provide rather extensive synonymy and citations because if you do not have access to the Taxapad database (Yu et al. 2012) it can be very difficult to trace the fate of names in the voluminous and scattered literature. Conventions and abbreviations are listed below.

[species] taxon deleted from the British and Irish list

BMNH Natural History Museum, London

NMS National Museums of Scotland, Edinburgh

\# known introductions occurring only under artificial conditions

? status (including uncertain synonymy) or identification in the British Isles uncertain

misident. has been misidentified as this name

nom. dub. nomen dubium, a name of doubtful status

nom. ob. nomen oblitum, 'forgotten name', does not have priority over a younger name

nom. nov. nomen novum, a replacement name

nom. nud. nomen nudum, an unavailable name, with no type specimen

preocc. name preoccupied (junior homonym)

stat. rev. status revocatus, revived status (e.g., raised from synonymy)

unavailable name unavailable under provisions of the ICZN code

var. variety, only available as a valid name under certain provisions of the ICZN code

Word document and spreadsheet versions of the checklist are available in the supplementary materials. Future updates to the British and Irish list will be incorporated in an online version of the checklist at Hymenoptera of the British Isles. 


\section{Agathidinae}

\section{Subfamily Agathidinae Haliday, 1833}

Notes: Except for Agathis and 'Bassus' species (i.e. including Lytopylus and Therophilus), distribution and synonymic data from Nixon (1986).

Tribe Agathidini Nees, 1814

Nomenclature:

BASSINI Nees, 1812 invalid

EUMICRODINI Förster, 1863

BASSINI Förster, 1869 preocc.

MICRODINI Ashmead, 1900

MESOCOELINI Viereck, 1918

ANEUROBRACONINI Fahringer, 1936

EARININI Sharkey, 1992

\section{Genus Agathis Latreille, 1804}

Nomenclature:

AENIGMOSTOMUS Ashmead, 1900

METRIOSOMA Szépligeti, 1902

BAEOGNATHA Kokujev, 1903 synonymy by Achterberg (2011)

LISSAGATHIS Cameron, 1911

RHAMPHAGATHIS Tobias, 1962

Notes: Distribution and synonymic data from Nixon (1986) and Simbolotti and Achterberg (1999).

Species of Agathis excluded from the British and Irish list:

[malvacearum Latreille, 1805; syn. panzeri (Jurine, 1807, Ichneumon); metzneriae Muesebeck, 1967] Listed by Huddleston (1978); probably refers to varipes.

\section{Agathis anglica Marshall, 1885}

Nomenclature:

longicauda Kokujev, 1895 preocc.

?marshalli Fahringer, 1937 
albanica Fischer, 1957

syriaca Fischer, 1957

caucasica Tobias, 1963

taiwanensis Chou \& Sharkey, 1989

Distribution: England, Wales

\section{Agathis assimilis Kokujev, 1895}

Nomenclature:

propinqua Kokujev, 1895

jakowlewi Kokujev, 1895

sibirica Telenga, 1933

anchisiades Nixon, 1986

Distribution: Scotland

Notes: added by Nixon (1986)

\section{Agathis breviseta Nees, 1812}

Nomenclature:

achterbergi Nixon, 1986

Distribution: England, Scotland, Ireland

Notes: Simbolotti and Achterberg (1999) synonymised Agathis achterbergi Nixon, 1986 under breviseta but it is listed as a valid species in Taxapad (Yu et al. 2012).

\section{Agathis fuscipennis (Zetterstedt, 1838)}

\section{Nomenclature:}

Microgaster fuscipennis Zetterstedt, 1838

breviseta misident.

rostrata misident.

glabricula Thomson, 1895

schmiedeknechti Kokujev, 1895

annulata Fahringer, 1937

meridionellae Fischer, 1957

albicostellae Fischer, 1966

artemesiana Fischer, 1966 
Distribution: England, Ireland

Agathis griseifrons Thomson, 1895

Nomenclature:

laticarpa Telenga, 1955

Distribution: England, Ireland

Agathis lugubris (Förster, 1863)

Nomenclature:

Cenostomus lugubris Förster, 1863

minuta Niezabitowski, 1910

Distribution: England, Scotland, Wales, Ireland

Notes: added by Nixon (1986)

Agathis montana Shestakov, 1932

Nomenclature:

zaykovi Nixon, 1986

Distribution: England

Notes: added by Simbolotti and Achterberg (1999)

Agathis nigra Nees, 1812

Nomenclature:

testaceipes Fischer, 1957

kasachstanica Tobias, 1963

nixoni Belokobylskij \& Jervis, 1998

Distribution: England

Agathis rufipalpis Nees, 1812

Distribution: Ireland

Agathis tibialis Nees, 1814

Nomenclature:

genualis Marshall, 1898 
Distribution: England

Notes: NMS, det. van Achterberg, added here

\section{Agathis varipes Thomson, 1895}

\section{Nomenclature:}

simulatrix Kokujev, 1895

rufipes Ivanov, 1899

dissimilis Shestakov, 1928

rufilabialis Fahringer, 1937

glabricollis Telenga, 1957

serratulae Tobias, 1963

lederi Fischer, 1968

ariadne Nixon, 1986

Distribution: England

Notes: added by Nixon (1986)

\section{Genus Bassus Fabricius, 1804}

\section{Nomenclature:}

MICRODUS Nees, 1814

DIPLOZON Haliday, 1833

EURYZONA Haliday, 1838 nom. nud.

EUMICRODUS Förster, 1863

HEMIOGASTER Enderlein, 1920 synonymy by Achterberg and Long (2010)

Notes: Following molecular phylogenetic analysis of Agathidinae (Sharkey et al. 2006), the genus Bassuss.l. was recognised as being a polyphyletic assemblage. Species are now being described in or reassigned to Lytopylus, Thermophilus and the non-British Camptothlipsis (Sharkey et al. 2009, Stevens et al. 2010, Stevens et al. 2011, Achterberg and Long 2010, Achterberg 2011) but very few European species have been formally transferred. The species on the British list have therefore been reassigned here on the bases of recent generic keys (e.g. Sharkey et al. 2009) in anticipation of future taxonomy. Whereas Bassuss. s. is now restricted to a small group of species (only one in Britain), Therophilus remains large and probably para- or polyphyletic. Distribution and synonymic data for Bassus, Lytopylus and Therophilus from Nixon (1986) and Simbolotti and Achterberg (1992). 


\section{Bassus calculator (Fabricius, 1798)}

Nomenclature:

Ichneumon calculator Fabricius, 1798

abscissus (Ratzeburg, 1844, Microdus)

Distribution: England

\section{Genus Earinus Wesmael, 1837}

Nomenclature:

Förster, 1863

\section{Earinus elator (Fabricius, 1804)}

Nomenclature:

Banchus elator Fabricius, 1804

nitidulus (Nees, 1814, Microdus)

thoracicus (Nees, 1834, Microdus)

major (Fonscolombe, 1846, Agathis)

pilosus Tobias, 1960

Distribution: England, Scotland, Ireland

\section{Earinus gloriatorius (Panzer, 1809)}

\section{Nomenclature:}

Bassus gloriatorius Panzer, 1809

gloriator (Nees, 1812, Microdus)

ochropes (Curtis, 1829, Microdus) nom. nud.

affinis (Wesmael, 1837, Microdus)

delusor (Wesmael, 1837, Microdus)

tuberculatus (Wesmael, 1837, Microdus)

varicoxis (Wesmael, 1837, Microdus)

niger (Zetterstedt, 1838, Microgaster)

bicingulatus (Thomson, 1895, Agathis)

ochropes Lyle, 1920

ruficoxis Fahringer, 1937

Distribution: England, Scotland, Ireland, Isle of Man 
Earinus transversus Lyle, 1920

Distribution: England

Notes: distribution data from Shaw (2005)

\section{Genus Lytopylus Förster, 1863}

\section{Nomenclature:}

AEROPHILUS Szépligeti, 1902 synonymy by Sharkey et al. (2006)

NEOMICRODUS Szépligeti, 1908

AEROPHILOPSIS Viereck, 1913

AEROPHILINA Enderlein, 1920

IOXIA Enderlein, 1920

HORMAGATHIS Brues, 1926

OBESOMICRODUS Papp, 1971

FACILAGATHIS van Achterberg \& Chen, 2004

Notes: See comments under Bassus; generic synonymy from Sharkey et al. (2009).

\section{Lytopylus rufipes (Nees, 1814)}

Nomenclature:

Microdus rufipes Nees, 1814

germanicus (Enderlein, 1904, Braunsia)

diversus Muesebeck, 1933

amurensis (Shestakov, 1940, Microdus)

Distribution: England

\section{Genus Therophilus Wesmael, 1837}

Nomenclature:

ORGILONEURA Ashmead, 1900

AGATHIELLA Szépligeti, 1902

AEROPHILIODES Strand, 1911

Notes: See comments under Bassus; generic synonymy from Sharkey et al. (2009).

Species excluded from the British and Irish list: 
[brevicaudis (Reinhard, 1867, Microdus)] Listed as a 'species inquirendae' by Nixon (1986) but included as a German species by Simbolotti and Achterberg (1992). Transferred to Bassus by Simbolotti and Achterberg (1999) but not listed as a species of Therophilus by Sharkey and Stoelb (2012).

[nugax (Reinhard, 1867, Microdus); syn. rufiventris (Abdinbekova, 1975, Microdus)] Listed by Huddleston (1978) but no mention of British specimens by Nixon (1986) or Simbolotti and Achterberg (1992).

\section{Therophilus arcuatus (Reinhard, 1867)}

Nomenclature:

Microdus arcuatus Reinhard, 1867

Notes: Taken out of synonymy with conspicuus by Simbolotti and Achterberg (1992).

Therophilus cingulipes (Nees, 1812)

Nomenclature:

Microdus cingulipes Nees, 1812

nantouensis Chou \& Sharkey, 1989

Distribution: England, Ireland

Therophilus clausthalianus (Ratzeburg, 1844)

Nomenclature:

Ichneumon clausthalianus Ratzeburg, 1844

Distribution: England, Ireland

Therophilus conspicuus (Wesmael, 1837)

Nomenclature:

Microdus conspicuus Wesmael, 1837

zonatus (Marshall, 1885, Earinus)

carpocapsae Cushman, 1915

angustatus (Telenga, 1955, Microdus)

variabilis Chou \& Sharkey, 1989

Distribution: England, Ireland 
Therophilus tegularis (Thomson, 1895)

Nomenclature:

Agathis tegularis Thomson, 1895

Distribution: England

Notes: added by Simbolotti and Achterberg (1992)

Therophilus tumidulus (Nees, 1812)

Nomenclature:

Microdus tumidulus Nees, 1812

intermedius (Ivanov, 1899, Eumicrodus)

annae (Enderlein, 1908, Microdus)

aino (Watanabe, 1937, Microdus)

ruficoxis (Fahringer, 1937, Microdus)

rufus (Fahringer, 1938, Microdus)

bicolor (Shestakov, 1940, Microdus) preocc.

victoris (Telenga, 1955, Microdus)

shestakovi (Shenefelt, 1970, Agathis)

anuphrievi (Tobias, 1986, Microdus)

Distribution: England, Scotland, Ireland

\section{Genus Therophilus Wesmael, 1837}

Notes: Species incertae sedis within Agathidinae; Sharkey and Stoelb (2012) defined Therophilus as a monophyletic genus and listed the included species, but several species currently classified in Therophilus are of uncertain generic placement.

\section{? Therophilus dimidiator (Nees, 1834)}

Nomenclature:

Microdus dimidiator Nees, 1834

cingulator (Ratzeburg, 1852, Microdus)

laticinctus (Cresson, 1873, Microdus)

ocellanae (Richardson, 1913, Microdus)

Distribution: England 
Notes: Listed by Huddleston (1978) but no mention of British specimens by Nixon (1986), who states, however, that the traditional interpretation of the species is probably correct. No British or Irish specimens were seen by Simbolotti and Achterberg (1992) but van Achterberg has identified an English specimen in BMNH as Therophilus dimidiator.

\section{? Therophilus epinotiae (van Achterberg, 1992)}

Nomenclature:

Bassus epinotiae van Achterberg, 1992

Distribution: England

Notes: added by Simbolotti and Achterberg (1992)

\section{? Therophilus linguarius (Nees, 1812)}

Nomenclature:

Microdus linguarius Nees, 1812

minor (Enderlein, 1908, Microdus)

kaszabi (Papp, 1967, Vipio)

Distribution: England

\section{? Therophilus mediator (Nees, 1814)}

Nomenclature:

Microdus mediator Nees, 1814

lugubrator (Ratzeburg, 1852, Microdus)

Distribution: England

Notes: Transferred from Agathis by Simbolotti and Achterberg (1999).

\section{? Therophilus pumilus (Ratzeburg, 1844)}

Nomenclature:

Microdus pumilus Ratzeburg, 1844

Distribution: England

Notes: NMS, det. Shaw, added here; listed by Huddleston (1978) but no mention of British specimens by Nixon (1986) or Simbolotti and Achterberg (1992). Transferred from Agathis by Simbolotti and Achterberg (1999) but listed as a species of Agathis in Taxapad (Yu et al. 2012). 
? Therophilus rugulosus (Nees, 1834)

Nomenclature:

Microdus rugulosus Nees, 1834

compeditus (Vollenhoven, 1878, Microdus)

punctatus (Abdinbekova, 1975, Microdus)

Distribution: England, Ireland

\section{Alysiinae}

Subfamily Alysiinae Leach, 1815

Tribe Alysiini Leach, 1815

Nomenclature:

ALLOEINI Ashmead, 1900

Genus Adelurola Strand, 1928

Nomenclature:

ADELURA Förster, 1863 preocc.

NEOCARPA Fischer, 1966

Adelurola florimela (Haliday, 1838)

Nomenclature:

Alysia florimela Haliday, 1838

multiarticulata (Marshall, 1898, Phaenocarpa)

pentapleuroides (Fischer, 1971, Dapsilarthra) unavailable

Distribution: England, Scotland, Ireland, Isle of Man

\section{Genus Alloea Haliday, 1833}

Nomenclature:

DIASPASTA Förster, 1863

LAMADATHA Cameron, 1900 
Alloea contracta Haliday, 1833

Nomenclature:

testaceipes (Cameron, 1900, Lamadatha)

Distribution: England, Scotland, Wales, Ireland

\section{Alloea lonchopterae Fischer, 1966}

Distribution: England, Isle of Man

Notes: Added by Godfray and Bland (2011) English record from specimen in BMNH.

Genus Alysia Latreille, 1804

Subgenus Alysia Latreille, 1804

Nomenclature:

CECHENUS Illiger, 1807

BASSUS Nees, 1812

GONIARCHA Förster, 1863

STROPHAEA Förster, 1863

Notes: Much of the taxonomy and distribution from Wharton (1986).

Species of Alysia (Alysia) excluded from the British and Irish list:

[cingulata Nees, 1834 nom. dub.]

Alysia (Alysia) alticola (Ashmead, 1890)

Nomenclature:

Pentapleura alticola Ashmead, 1890

soror Marshall, 1894

Distribution: England, Scotland, Wales, Isle of Man

Alysia (Alysia) frigida Haliday, 1838

Distribution: England

Notes: added by Godfray and Achterberg (2015) 
Alysia (Alysia) incongrua Nees, 1834

Distribution: England, Scotland, Ireland

Alysia (Alysia) lucia Haliday, 1838

Nomenclature:

diversiceps Fischer, 1967

Distribution: Scotland

Alysia (Alysia) lucicola Haliday, 1838

Distribution: England, Scotland, Ireland

Alysia (Alysia) luciella Stelfox, 1941

Distribution: Ireland

Alysia (Alysia) manducator (Panzer, 1799)

Nomenclature:

Ichneumon manducator Panzer, 1799

haematopa (Gmelin, 1790, Ichneumon) preocc.

stercoraria Latreille, 1805

apicalis Curtis, 1826

similis Curtis, 1826

stercorator Lamarck, 1835

curtungula Thomson, 1895

bucephala Marshall, 1898

manducatrix Schulz, 1906

Distribution: England, Scotland, Wales, Ireland, Isle of Man

Alysia (Alysia) truncator (Nees, 1812)

Nomenclature:

Bassus truncator Nees, 1812

Distribution: England, Scotland, Ireland 


\section{Subgenus Anarcha Förster, 1863}

Notes: Much of the taxonomy and distribution from Wharton (1988b).

Species of Alysia (Anarcha) excluded from the British and Irish list:

[similis (Nees, 1812, Bassus) nom. dub.]

\section{Alysia (Anarcha) atra Haliday, 1838}

Distribution: Ireland

Alysia (Anarcha) fuscipennis Haliday, 1838

Nomenclature:

obscuripes Thomson, 1895

Distribution: England, Scotland, Ireland

Alysia (Anarcha) mandibulator (Nees, 1812)

Nomenclature:

Bassus mandibulator Nees, 1812

loripes Haliday, 1838

mandibulatrix Schulz, 1906

Distribution: England, Ireland

Alysia (Anarcha) rufidens Nees, 1834

Nomenclature:

puncticollis Thomson, 1895

Distribution: England, Ireland

Alysia (Anarcha) sophia Haliday, 1835

Distribution: Ireland

Alysia (Anarcha) subaperta Thomson, 1895

Nomenclature:

similis misident.

Distribution: England 
Notes: added by Wharton (1988b)

Alysia (Anarcha) thapsina Wharton, 1988

Distribution: Ireland

Notes: added by Wharton (1988b)

Alysia (Anarcha) tipulae (Scopoli, 1763)

Nomenclature:

Ichneumon tipulae Scopoli, 1763

abdominator (Nees, 1814, Bassus)

notabilis (Förster, 1863, Anarcha)

Distribution: England, Scotland, Ireland

Alysia (Anarcha) umbrata Stelfox, 1941

Distribution: Ireland

Genus Anisocyrta Förster, 1863

Anisocyrta perdita (Haliday, 1838)

Nomenclature:

Alysia perdita Haliday, 1838

Distribution: Scotland

\section{Genus Aphaereta Förster, 1863}

Nomenclature:

TRICHESIA Provancher, 1880

TRINARIA Provancher, 1886

APHAERETE Dalla Torre, 1898

ATOPANDRIUM Graham, 1952

TRISYNALDIS Fischer, 1958

Notes: Atopandrium was synonymised under Aphaereta by Achterberg (1995) but treated as a valid genus again in Taxapad (Yu et al. 2012). 


\section{Aphaereta debilitata Morley, 1933}

Nomenclature:

loripenne (Graham, 1952, Atopandrium)

confluctum (Fischer, 1958, Trisynaldis)

Distribution: England, Wales

\section{Aphaereta falcigera Graham, 1960}

Distribution: England, Ireland

Aphaereta major (Thomson, 1895)

Nomenclature:

Alysia major Thomson, 1895

major Marshall, 1898 preocc.

Distribution: England, Scotland, Ireland

\section{Aphaereta minuta (Nees, 1811)}

Nomenclature:

Stephanus minutus Nees, 1811

cephalotes (Haliday, 1833, Alysia)

fuscipes (Nees, 1834, Alysia)

confluens (Ratzeburg, 1844, Alysia)

stigmaticalis (Thomson, 1895, Alysia)

inepta Morley, 1933

Distribution: England, Scotland, Ireland

\section{Aphaereta pallipes (Say, 1829)}

Nomenclature:

Alysia pallipes Say, 1829

auripes (Provancher, 1881, Trichesia)

pilicornis (Provancher, 1886, Trinaria)

californica Ashmead, 1889

muscae Ashmead, 1889

oscinidis Ashmead, 1889 
pallidipes (Dalla Torre, 1898, Aphaerete)

delosa Viereck, 1905

subtricarinata Viereck, 1905

pegomyiae Brues, 1907

sarcophagae Gahan, 1914

Distribution: England

Notes: added by Shaw (1983)

Aphaereta tenuicornis Nixon, 1939

Distribution: England, Wales, Ireland

\section{Genus Asobara Förster, 1863}

Nomenclature:

SPANISTA Förster, 1863

\section{Asobara tabida (Nees, 1834)}

Nomenclature:

Alysia tabida Nees, 1834

anomala (Thomson, 1895, Alysia)

crenulata (Fahringer, 1935, Phaenocarpa)

Distribution: England, Ireland

\section{Genus Aspilota Förster, 1863}

Nomenclature:

DIPIESTA Förster, 1863

EUSYNALDIS Zaykov \& Fischer, 1982

SYNALDIS misident.

Notes: Some distribution data from Stelfox and Graham (1951a). Some species treated as belonging to Dinotrema by other authors have been included in Aspilota in Taxapad (Yu et al. 2012) and there is clearly much work to be done in allocating species to the current generic concepts (see Wharton 1985). The late T. Munk was preparing a revision of the European species of Aspilotas. I. (see note under Dinotrema). 
Aspilota acutidentata (Fischer, 1970)

Nomenclature:

Synaldis acutidentata Fischer, 1970

Distribution: England, Scotland

Notes: NMS, BMNH, det. Munk, added here; identified by T. Munk as a species of Dinotrema.

Aspilota anaphoretica Fischer, 1973

Distribution: England, Scotland, Ireland

Notes: NMS, BMNH, det. Munk, added here

Aspilota blasii Fischer, 1973

Distribution: England

Notes: BMNH, det. Munk, added here

Aspilota compressiventris Stelfox \& Graham, 1951

Distribution: England

Aspilota curta Marshall, 1895

Distribution: England

Aspilota daemon Stelfox and Graham, 1948

Distribution: England, Scotland, Ireland

Aspilota delicata Fischer, 1973

Distribution: England, Scotland, Ireland

Notes: BMNH, det. Munk, added here

Aspilota efoveolata Thomson, 1895

Nomenclature:

pneumatica Fischer, 1973

Distribution: England, Scotland, Wales 
Notes: NMS, BMNH, det. Munk, added here

\section{Aspilota flagellaris Fischer, 1973}

Distribution: England, Scotland, Ireland

Notes: BMNH, det. Munk, added here

Aspilota fuscicornis (Haliday, 1838)

Nomenclature:

Alysia fuscicornis Haliday, 1838

?minuta (Nees, 1812, Bassus)

exile (Ruthe, 1859, Orthostigma)

dilatata (Thomson, 1895, Alysia)

Distribution: England, Scotland, Ireland

\section{Aspilota globipes (Fischer, 1962)}

Nomenclature:

Synaldis globipes Fischer, 1962

Distribution: England, Scotland

Notes: NMS, BMNH, det. Munk, added here

\section{Aspilota imparidens Fischer, 1974}

Distribution: England, Scotland, Wales

Notes: NMS, BMNH, det. Munk, added here

\section{Aspilota insolita (Tobias, 1962)}

Nomenclature:

Orthostigma insolita Tobias, 1962

Distribution: England, Ireland

Notes: BMNH, det. Munk, added here

Aspilota intermediana Fischer, 1975

Distribution: England 
Notes: added by Notton (1991)

Aspilota iocosipecta Fischer, 1974

Distribution: England, Scotland, Ireland

Notes: NMS, BMNH, det. Munk, added here

Aspilota macrops Stelfox \& Graham, 1951

Distribution: England, Ireland

Aspilota nidicola Hedqvist, 1972

Distribution: England

Notes: BMNH, det. Munk, added here

Aspilota pillerensis Fischer, 1973

Distribution: England

Notes: BMNH, det. Munk, added here

Aspilota ruficornis (Nees, 1834)

Nomenclature:

Alysia ruficornis Nees, 1834

Distribution: England, Scotland, Wales, Ireland

Aspilota stenogaster Stelfox \& Graham, 1951

Distribution: England

Aspilota tetragona Fischer, 1976

Distribution: England, Scotland, Ireland

Notes: NMS, BMNH, det. Munk, added here

Aspilota vernalis Stelfox \& Graham, 1951

Distribution: England, Scotland, Ireland 


\section{Genus Chasmodon Haliday, 1838}

\section{Chasmodon apterus (Nees, 1812)}

Nomenclature:

Bassus apterus Nees, 1812

Distribution: England, Scotland, Wales, Ireland, Isle of Man

\section{Genus Cratospila Förster, 1863}

Nomenclature:

HEDYLUS Marshall, 1891; synonymy by Papp (2009b)

\section{Cratospila circe (Haliday, 1838)}

Nomenclature:

Alysia circe Haliday, 1838

habilis (Marshall, 1891, Hedylus); synonymy by Papp (2009b)

annellata (Thomson, 1895, Alysia)

Distribution: England, Ireland

\section{Genus Dapsilarthra Förster, 1863}

Notes: Dapsilarthra has been used in a very broad sense to include Adelurola, Mesocrina, Heterolexis and Grammospila, and in a more narrow sense to include the last two genera. Achterberg (2014) is followed here in according each generic rank.

\section{Dapsilarthra apii (Curtis, 1826)}

Nomenclature:

Alysia apii Curtis, 1826

laevipectus (Thomson, 1895, Alysia)

americana (Brues, 1907, Orthostigma)

Distribution: England, Scotland, Ireland

\section{Dapsilarthra sylvia (Haliday, 1839)}

Nomenclature:

Alysia sylvia Haliday, 1839

carpathica van Achterberg, 1983; synonymy by Achterberg (1997) 
Distribution: England, Scotland, Ireland

\section{Genus Dinotrema Förster, 1863}

Notes: Wharton (1980) summarises the arguments against recognition of the genus Synaldis; Achterberg (1988b) likewise notes that the genus is defined only by the absence of fore wing vein 2-SR, which is known to be intraspecifically variable (Wharton 1980). Despite this, various authors attach great taxonomic weight to this venational character and maintain Synaldis as a valid genus (as reflected by the classification in $\mathrm{Yu}$ et al. 2012). Species that would be classified in Synaldis ( acutidentata, concolor, distracta and globipes) are listed here in Aspilota and Dinotrema , according to Fauna Europaea. Some distribution data from Stelfox and Graham (1951b), Stelfox and Graham (1951a) and Achterberg (1988a). The late T. Munk was revising the European species of Aspilotas.l. and gave much helpful advice on the generic placements of the species occurring in Britain and Ireland, some of which is unpublished and will therefore differ from the generic combinations found in, e.g. Belokobylskij et al. (2003) and Taxapad (Yu et al. 2012). Munk also advised that although Aspilota is a well-defined genus, Dinotrema is not defined by any apomorphies and will be split up.

Species excluded from the British and Irish list:

[pusillum (Nees, 1812, Bassus)] T. Munk (pers. comm.) regarded this as an unidentified species; the type has been destroyed.

\section{Subgenus Dinotrema Förster, 1863}

\section{Nomenclature:}

ASPILOTA misident.

SYNALDIS Förster, 1863

COLOBOMA Förster, 1863

SPANOMERIS Förster, 1863

SCOTIONEURUS Provancher, 1886

PTERUSA Fischer, 1958; synonymy by Achterberg and Vikberg (2014)

EUDINOSTIGMA Tobias, 1986; synonymy by Wharton (2002)

\section{Dinotrema (Dinotrema) aluum (Stelfox \& Graham, 1950)}

Nomenclature:

Aspilota alua Stelfox \& Graham, 1950

alva misspelling

Distribution: Ireland 
Dinotrema (Dinotrema) alysiae Munk \& Peris-Felipo, 2013

Distribution: England

Notes: added by Munk et al. (2013)

Dinotrema (Dinotrema) areolatum (Stelfox \& Graham, 1950)

Nomenclature:

Aspilota areolata Stelfox \& Graham, 1950

Distribution: England, Scotland

Dinotrema (Dinotrema) brevicorne (Nees, 1814)

Nomenclature:

Bassus brevicornis Nees, 1814

Distribution: England

Dinotrema (Dinotrema) brevissimicorne (Stelfox \& Graham, 1948)

Nomenclature:

Aspilota brevissimicornis Stelfox \& Graham, 1948

Distribution: Scotland, Ireland

Dinotrema (Dinotrema) compressum (Haliday, 1838)

Nomenclature:

Alysia compressa Haliday, 1838

Distribution: England

Dinotrema (Dinotrema) concinnum (Haliday, 1838)

Nomenclature:

Alysia concinna Haliday, 1838

maximum (Fischer, 1962, Synaldis)

tyrrhena (Masi, 1933, Aspilota)

Distribution: England, Ireland 
Notes: Synaldis maximum Fischer, 1962 has been treated as a junior synonym by König (1972) and subsequent authors but T. Munk (pers. comm.) regarded this as a valid species of Dinotrema that may be found to occur in Britain.

Dinotrema (Dinotrema) concolor (Nees, 1812)

Nomenclature:

Bassus concolor Nees, 1812

distractum (Ruthe, 1859, Orthostigma) unavailable

Distribution: England, Ireland

Dinotrema (Dinotrema) crassicosta (Thomson, 1895)

Nomenclature:

Alysia crassicosta Thomson, 1895

Distribution: Scotland

Notes: NMS, det. Munk, added here

Dinotrema (Dinotrema) cratocera (Thomson, 1895)

Nomenclature:

Alysia cratocera Thomson, 1895

Distribution: Scotland

Notes: BMNH, det. Munk, added here

Dinotrema (Dinotrema) denticulatum (Stelfox \& Graham, 1951)

Nomenclature:

Aspilota denticulata Stelfox \& Graham, 1951

Distribution: Ireland

Dinotrema (Dinotrema) dimidiatum (Thomson, 1895)

Nomenclature:

Alysia dimidiata Thomson, 1895

Distribution: England

Notes: BMNH, det. Munk, added here 
Dinotrema (Dinotrema) distractum (Nees, 1834)

Nomenclature:

Alysia distracta Nees, 1834

Dinotrema (Dinotrema) divisum (Stelfox \& Graham, 1950)

Nomenclature:

Aspilota divisa Stelfox \& Graham, 1950

?aureliae (Fischer, 1973, Aspilota); tentative synonymy by Papp (2004b)

Distribution: England, Scotland, Ireland

Dinotrema (Dinotrema) erythropum Förster, 1863

Nomenclature:

praecipuum (Marshall, 1895, Aspilota)

Distribution: England, Wales, Ireland

Dinotrema (Dinotrema) falsificum (Stelfox \& Graham, 1950)

Nomenclature:

Aspilota falsifica Stelfox \& Graham, 1950

Distribution: Scotland, Ireland

Dinotrema (Dinotrema) glabrum (Stelfox \& Graham, 1951)

Nomenclature:

Aspilota glabra Stelfox \& Graham, 1951

venustum (Tobias, 1962, Aspilota)

Distribution: England, Scotland, Ireland

Dinotrema (Dinotrema) insidiatrix (Marshall, 1895)

Nomenclature:

Aspilota insidiatrix Marshall, 1895

Distribution: England 
Dinotrema (Dinotrema) insignis (Stelfox \& Graham, 1950)

Nomenclature:

Aspilota insignis Stelfox \& Graham, 1950

Distribution: England

Dinotrema (Dinotrema) iuxtanaeviam (Fischer, 1980)

Nomenclature:

Aspilota iuxtanaeviam Fischer, 1978

Distribution: England

Notes: added by Fischer (1980)

Dinotrema (Dinotrema) jaculans (Haliday, 1838)

Nomenclature:

Alysia jaculans Haliday, 1838

Distribution: England, Scotland, Ireland

Dinotrema (Dinotrema) latistigma (Fischer, 1962)

Nomenclature:

Synaldis latistigma Fischer, 1962

Distribution: England

Notes: NMS, det. Munk, added here; treated as a species of Eudinostigma in Fauna Europaea and Taxapad (Yu et al. 2012).

Dinotrema (Dinotrema) lineola (Thomson, 1895)

Nomenclature:

Alysia lineola Thomson, 1895

Distribution: England

Dinotrema (Dinotrema) liosoma (Stelfox \& Graham,1951)

Nomenclature:

Aspilota liosoma Stelfox \& Graham,1951

caudatum (Thomson, 1895, Aspilota) preocc. 
Dinotrema (Dinotrema) mesocaudatum van Achterberg, 1988

Distribution: England

Notes: added by Achterberg (1988b)

Dinotrema (Dinotrema) microcera (Thomson, 1895)

Nomenclature:

Alysia microcera Thomson, 1895

Distribution: England, Scotland, Wales, Ireland

Notes: BMNH, det. Munk, added here

Dinotrema (Dinotrema) necrophilum (Hedqvist, 1972)

Nomenclature:

Aspilota necrophila Hedqvist, 1972

Distribution: England

Notes: Added by Disney and Munk (2005) and transferred from Aspilota.

Dinotrema (Dinotrema) nervosum (Haliday, 1833)

Nomenclature:

Alysia nervosa Haliday, 1833

Distribution: England, Scotland, Ireland

Dinotrema (Dinotrema) pulvinatum (Stelfox \& Graham, 1949)

Nomenclature:

Aspilota pulvinata Stelfox \& Graham, 1949

Distribution: England, Scotland, Wales, Ireland

Notes: Treated as a species of Eudinostigma in Fauna Europaea and Taxapad (Yu et al. 2012).

Dinotrema (Dinotrema) ruficollis (Stelfox \& Graham, 1950)

Nomenclature:

Aspilota ruficollis Stelfox \& Graham, 1950

Distribution: England, Ireland 
Notes: Distribution data from Achterberg (1988b).

Dinotrema (Dinotrema) semicompressum (Stelfox \& Graham, 1949)

Nomenclature:

Aspilota semicompressa Stelfox \& Graham, 1949

parapunctatum (Fischer, 1976, Aspilota); synonymy by Papp (2004b)

Distribution: England, Scotland, Ireland

Dinotrema (Dinotrema) sphaerimembre (Fischer, 1973)

Nomenclature:

Aspilota sphaerimembris Fischer, 1973

Distribution: Scotland

Notes: NMS, det. Munk, added here

Dinotrema (Dinotrema) sternaulicum (Fischer, 1973)

Nomenclature:

Aspilota sternaulica Fischer, 1973

Distribution: England, Scotland

Notes: NMS, det. Munk, added here

Dinotrema (Dinotrema) tauricum (Telenga, 1935)

Nomenclature:

Aspilota taurica Telenga, 1935

Distribution: England

Notes: added by Achterberg (1988b)

Dinotrema (Dinotrema) vesparum (Stelfox, 1943)

Nomenclature:

Aspilota vesparum Stelfox, 1943

Distribution: England, Scotland 
Dinotrema (Dinotrema) vituperatum (Fischer, 1974)

Nomenclature:

Aspilota vituperata Fischer, 1974

Distribution: England, Scotland

Notes: NMS, det. Munk, added here

\section{Subgenus Leptotrema van Achterberg, 1988}

Notes: Described as a separate genus by Achterberg (1988b), who gives distribution data for the one included species; treated as a subgenus of Dinotrema by Wharton (2002).

Dinotrema (Leptotrema) dentifemur (Stelfox, 1943)

Nomenclature:

Aspilota dentifemur Stelfox, 1943

Distribution: England, Scotland, Ireland

Subgenus Prosapha Förster, 1863

Dinotrema (Prosapha) speculum (Haliday, 1838)

Nomenclature:

Alysia speculum Haliday, 1838

venustum (Haliday, 1838, Alysia)

Distribution: England, Ireland

\section{Genus Grammospila Förster, 1863}

Nomenclature:

PARAORTHOSTIGMA Königsmann, 1972

Grammospila isabella (Haliday, 1838)

Nomenclature:

Alysia isabella Haliday, 1838

Distribution: England, Scotland 
Grammospila rufiventris (Nees, 1812)

Nomenclature:

Bassus rufiventris Nees, 1812

flaviventris (Haliday, 1838, Alysia)

ochrogaster (Szépligeti, 1898, Phaenocarpa)

fuscula Griffiths, 1968

Distribution: England, Scotland, Ireland, Isle of Man

Notes: Grammospila fuscula (Griffiths, 1968, Dapsilarthra) removed from synonymy by Achterberg (2014).

\section{Genus Heterolexis Förster, 1863}

Heterolexis balteata (Thomson, 1895)

Nomenclature:

Alysia balteata Thomson, 1895

Distribution: England, Scotland, Wales, Ireland

Heterolexis dictynna (Marshall, 1895)

Nomenclature:

Adelura dictynna Marshall, 1895

Distribution: England, Scotland, Ireland

Genus Idiasta Förster, 1863

Nomenclature:

EUPHAENOCARPA Tobias, 1975

Idiasta dichrocera Königsmann, 1960

Distribution: England

Notes: NMS, det. Shaw, added here

Idiasta maritima (Haliday, 1838)

Nomenclature:

Alysia maritima Haliday, 1838 
Distribution: England, Scotland, Ireland

Idiasta nephele (Haliday, 1838)

Nomenclature:

Alysia nephele Haliday, 1838

Distribution: England, Scotland

Genus Mesocrina Förster, 1863

Nomenclature:

PSEUDOMESOCRINA Königsmann, 1959

Mesocrina indagatrix Förster, 1863

Nomenclature:

venatrix Marshall, 1895

Distribution: England

Genus Orthostigma Ratzeburg, 1844

Nomenclature:

DELOCARPA Förster, 1863

ISCHNOCARPA Förster, 1863

Orthostigma cratospilum (Thompson, 1895)

Nomenclature:

Alysia cratospila Thompson, 1895

Distribution: England

Notes: added by Godfray and Achterberg (2015)

Orthostigma longicorne Königsmann, 1969

Distribution: Ireland

Orthostigma maculipes (Haliday, 1838)

Nomenclature:

Alysia maculipes Haliday, 1838

Distribution: England, Ireland 
Orthostigma pumilum (Nees, 1834)

Nomenclature:

Alysia pumila Nees, 1834

flavipes (Ratzeburg, 1844, Aphidius)

fulvipes Rondani, 1876 nom. nud.

brunnipes Ratzeburg, 1852

bruneipes Dalla Torre, 1898

Distribution: England, Ireland

\section{Genus Panerema Förster, 1863}

Notes: Distribution data from Achterberg (1988b).

Panerema fulvicornis (Haliday, 1838)

Nomenclature:

Alysia fulvicornis Haliday, 1838

Distribution: Wales, Ireland

Panerema inops Förster, 1863

Distribution: England, Wales, Ireland

\section{Genus Pentapleura Förster, 1863}

Nomenclature:

OPISENDEA Förster, 1863

GNATHOSPILA Fischer, 1966

Pentapleura angustula (Haliday, 1838)

Nomenclature:

Alysia angustula Haliday, 1838

laevipleuris (Tobias, 1962, Aspilota)

Distribution: England, Scotland, Ireland 
Pentapleura fuliginosa (Haliday, 1838)

Nomenclature:

Alysia fuliginosa Haliday, 1838

carinata (Thomson, 1895, Alysia)

Distribution: England, Wales, Ireland

Pentapleura pumilio (Nees, 1812)

Nomenclature:

Bassus pumilio Nees, 1812

triticaphis (Fitch, 1861, Toxares)

mesocrinoides Goidanich, 1936

Distribution: England, Scotland, Wales, Ireland

\section{Genus Phaenocarpa Förster, 1863}

Notes: Some taxonomic and distribution data from Achterberg (1997).

\section{Subgenus Homophyla Förster, 1863}

Notes: species of Phaenocarpa (Homophyla) excluded from the British and Irish list:

[pegomyiae Marshall, 1898] Listed as British by Lyle (1933) but we have not seen any British or Irish specimens.

Phaenocarpa (Homophyla) pullata (Haliday, 1838)

Nomenclature:

Alysia pullata Haliday, 1838

Distribution: England, Ireland

\section{Subgenus Phaenocarpa Förster, 1863}

Nomenclature:

IDIOLEXIS Förster, 1863; synonymy by Wharton (2002)

MESOTHESIS Förster, 1863

SATHRA Förster, 1863

ASYNAPHES Provancher, 1886

KAHLIA Ashmead, 1900 
HOLCALYSIA Cameron, 1905

STIRALYSIA Cameron, 1910

RHOPALONEURA Stelfox, 1941

Phaenocarpa (Phaenocarpa) canaliculata Stelfox, 1941

Distribution: England, Ireland

Phaenocarpa (Phaenocarpa) conspurcator (Haliday, 1838)

Nomenclature:

Alysia conspurcator Haliday, 1838

arctica (Thomson, 1895, Alysia)

tatrica Niezabitowski, 1910

remota Papp, 1981

Distribution: England, Scotland, Wales, Ireland

Phaenocarpa (Phaenocarpa) eugenia (Haliday, 1838)

Nomenclature:

Alysia eugenia Haliday, 1838

pectoralis (Zetterstedt, 1838, Alysia)

orbicularis Gurasashvilli, 1983

Distribution: England, Scotland, Ireland

Phaenocarpa (Phaenocarpa) eunice (Haliday, 1838)

Nomenclature:

Alysia eunice Haliday, 1838

nimia Stelfox, 1941

Distribution: England, Wales, Ireland

Phaenocarpa (Phaenocarpa) flavipes (Haliday, 1838)

Nomenclature:

Alysia flavipes Haliday, 1838

Distribution: England, Ireland 
Phaenocarpa (Phaenocarpa) frequentator (Zetterstedt, 1838)

Nomenclature:

Alysia frequentator Zetterstedt, 1838

frequentatrix (Schulz, 1906, Alysia)

Distribution: Wales

Notes: added by Godfray and Achterberg (2015)

Phaenocarpa (Phaenocarpa) galatea (Haliday, 1838)

Nomenclature:

Alysia galatea Haliday, 1838

Distribution: Scotland, Ireland

Phaenocarpa (Phaenocarpa) helophilae van Achterberg, 1998

Distribution: England

Notes: added by Achterberg (1998)

Phaenocarpa (Phaenocarpa) livida (Haliday, 1838)

Nomenclature:

Alysia livida Haliday, 1838

debilis (Förster, 1863, Sathra)

Distribution: England, Ireland

Phaenocarpa (Phaenocarpa) luteipes Stelfox, 1950

Distribution: Ireland

Phaenocarpa (Phaenocarpa) maria (Haliday, 1838)

Nomenclature:

Alysia maria Haliday, 1838

Distribution: England, Ireland

Phaenocarpa (Phaenocarpa) nina (Haliday, 1838)

Nomenclature:

Alysia nina Haliday, 1838 
Distribution: Scotland

Notes: Not listed in Huddleston (1978), although described from Scottish material (Achterberg 1998).

Phaenocarpa (Phaenocarpa) notabilis Stelfox, 1944

Distribution: Scotland, Ireland

Phaenocarpa (Phaenocarpa) picinervis (Haliday, 1838)

Nomenclature:

Alysia picinervis Haliday, 1838

americana Ashmead, 1889

Distribution: England, Scotland, Ireland

Phaenocarpa (Phaenocarpa) pratellae (Curtis, 1826)

Nomenclature:

Alysia pratellae Curtis, 1826

piceator (Zetterstedt, 1838, Alysia)

psalliotae Telenga, 1935; synonymy by Achterberg (2014)

Distribution: England, Scotland

Phaenocarpa (Phaenocarpa) punctigera (Haliday, 1838)

Nomenclature:

Alysia punctigera Haliday, 1838

Distribution: England, Ireland

Notes: Listed as a species of Idiolexis in Fauna Europaea.

Phaenocarpa (Phaenocarpa) ruficeps (Nees, 1812)

Nomenclature:

Bassus ruficeps Nees, 1812

testacea (Nees, 1812, Bassus)

gracilis (Curtis, 1826, Alysia)

pallida (Curtis, 1826, Alysia)

agricolator (Zetterstedt, 1838, Alysia) 


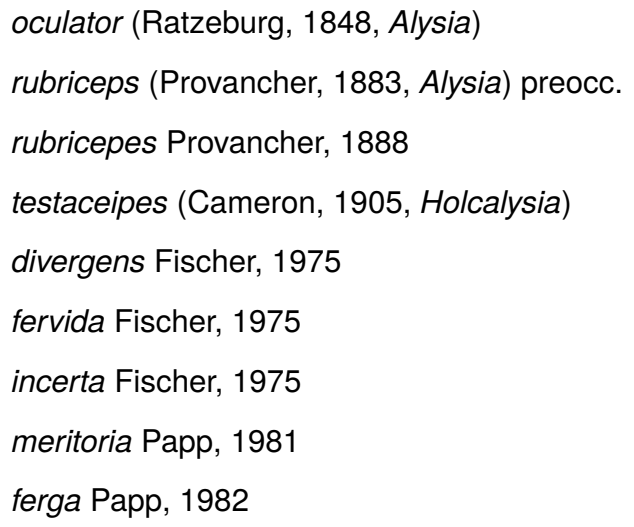

Distribution: England, Scotland, Wales, Ireland, Isle of Man

Phaenocarpa (Phaenocarpa) tacita Stelfox, 1941

Nomenclature:

caucasica Gurasashvili, 1983 preocc.

caucasicola Tobias, 1986

Distribution: Ireland

Phaenocarpa (Phaenocarpa) trisulcata Stelfox, 1950

Distribution: Ireland

\section{Genus Syncrasis Förster, 1863}

Nomenclature:

PHAENOLYTA Förster, 1863

Syncrasis fucicola (Haliday, 1838)

Nomenclature:

Alysia fucicola Haliday, 1838

Distribution: Ireland

Syncrasis halidayi (Förster, 1863)

Nomenclature:

Phaenolyta halidayi Förster, 1863

fuscipes preocc., unavailable 
Distribution: England, Ireland

\section{Genus Tanycarpa Förster, 1863}

Nomenclature:

ACROBELA Förster, 1863; synonymy by Wharton (2002)

EPICLISTA Förster, 1863

HYPOSTROPHA Förster, 1863

Tanycarpa bicolor (Nees, 1812)

Nomenclature:

Bassus bicolor Nees, 1812

ancilla (Haliday, 1838, Alysia)

Distribution: England, Ireland

Tanycarpa gracilicornis (Nees, 1812)

Nomenclature:

Bassus gracilicornis Nees, 1812

Distribution: Ireland

Tanycarpa mitis Stelfox, 1941

Distribution: England, Ireland

Tanycarpa punctata van Achterberg, 1976

Tanycarpa rufinotata (Haliday, 1838)

Nomenclature:

Alysia rufinotata Haliday, 1838

carinata (Förster, 1863, Acrobela)

erythrogaster (Förster, 1863, Epiclista)

foersteri (Shenefelt, 1974, Alysia)

Distribution: England, Ireland 


\section{Genus Trachyusa Ruthe, 1854}

Nomenclature:

COSMIOCARPA Förster, 1863

Notes: Distribution data from Achterberg and O'Connor (1990) and the collections of NMS.

\section{Trachyusa aurora (Haliday, 1838)}

Nomenclature:

Alysia aurora Haliday, 1838

nigriceps Ruthe, 1854

Distribution: England, Ireland

\section{Trachyusa nigrothoracica van Achterberg \& O’Connor, 1990}

Distribution: England, Ireland

Notes: added by Achterberg and O'Connor (1990)

\section{Tribe Dacnusini Förster, 1863}

Notes: The classification of the Dacnusini was revised by Griffiths (Griffiths 1964, Griffiths 1967a, Griffiths 1967b, Griffiths 1967c, Griffiths 1968a, Griffiths 1968b) in a pioneering treatment that was one of the first applications of explicit phylogenetic methods in taxonomy. It was also unusual in being based on extensive reared material. Griffiths' classification is followed here although one of the consequences of his strict adherence to phylogenetic principles is that Chorebus and Dacnusa are very large genera. Also, as Griffiths realised, the definition of Exotela is unsatisfactory as it lacks clear apomorphic characters. This group of insects had previously been revised by Nixon (Nixon 1943, Nixon 1944, Nixon 1945, Nixon 1946, Nixon 1948, Nixon 1949, Nixon 1954), who recognised a greater number of genera, and some authors (Tobias 1986, Tobias 1998, Perepechayenko 2000, Fischer et al. 2004) have resurrected Nixon's genera or used them as subgenera. This seems a retrograde step given Griffiths' clear statements of phylogenetic hypotheses and is not used here. In both Chorebus and Dacnusa, Griffiths defined a series of species groups that with further research should form the basis of better classifications of these genera. Griffiths (1964) gives a key to genera and Wharton (1997) is also very helpful. The Coelinius genus group (Coelinius, Trachionus, Epimicta, Aristelix, Laotris, Sarops and Synelix) is generally agreed to be monophyletic and was not revised at species level by Nixon or Griffiths. Distribution data from Griffiths' and Nixon's revisions (loc. cit.) and NMS. 
Genus Amyras Nixon, 1943

Amyras clandestina (Haliday, 1839)

Nomenclature:

Alysia clandestina Haliday, 1839

quadridentata (Thomson, 1895, Dacnusa)

Distribution: Ireland

Genus Aristelix Nixon, 1943

Aristelix phaenicura (Haliday, 1839)

Nomenclature:

Alysia phaenicura Haliday, 1839

phoenicura misspelling

Distribution: England, Ireland

Genus Chaenusa Haliday, 1839

Nomenclature:

CHOREBIDEA Viereck, 1914

CHOREBIDEA Nixon, 1943 preocc.

CHOREBIDELLA Riegel, 1947

Notes: No recent revision of this genus.

Chaenusa conjungens (Nees, 1811)

Nomenclature:

Bracon conjungens Nees, 1811

conjugens misspelling

Distribution: England, Scotland, Ireland

Chaenusa elongata Stelfox, 1957

Distribution: Ireland 
Chaenusa limoniadum (Marshall, 1896)

Nomenclature:

Chorebus limoniadum Marshall, 1896

Distribution: England

Notes: Transferred from Chorebus by Perepechayenko (2000).

Chaenusa lymphata (Haliday, 1839)

Nomenclature:

Alysia lymphata Haliday, 1839

Distribution: England, Ireland

Notes: Transferred from Chorebus by Achterberg (1997).

Chaenusa naiadum (Haliday, 1839)

Nomenclature:

Alysia naiadum Haliday, 1839

naiadum (Curtis, 1837, Chorebus) nom. nud.

Distribution: England, Scotland, Ireland

Chaenusa nereidum (Haliday, 1839)

Nomenclature:

Alysia nereidum Haliday, 1839

nereidum (Curtis, 1837, Chorebus) nom. nud.

Distribution: England, Ireland

Chaenusa opaca Stelfox, 1957

Distribution: Ireland

\section{Genus Chorebus Haliday, 1833}

Nomenclature:

AMETRIA Förster, 1863

GYROCAMPA Förster, 1863

PHAENOLEXIS Förster, 1863

STIPHROCERA Förster, 1863 
DIPLUSIA Ruthe, 1882

ETRIPTES Nixon, 1943

PARAGYROCAMPA Tobias, 1962

Chorebus abaris (Nixon, 1943)

Nomenclature:

Dacnusa abaris Nixon, 1943

Distribution: England, Scotland

Chorebus abnormiceps (Nixon, 1943)

Nomenclature:

Dacnusa abnormiceps Nixon, 1943

quadriceps (Nixon, 1941, Dacnusa) preocc.

Distribution: England

Chorebus agraules (Nixon, 1945)

Nomenclature:

Dacnusa agraules Nixon, 1945

Distribution: England

Chorebus albipes (Haliday, 1839)

Nomenclature:

Alysia albipes Haliday, 1839

Distribution: England, Scotland, Ireland

Chorebus alecto (Morley, 1924)

Nomenclature:

Rhizarcha alecto Morley, 1924

turissa (Nixon, 1937, Dacnusa)

Distribution: England, Scotland, Ireland

Chorebus alua (Nixon, 1944)

Nomenclature:

Dacnusa alua Nixon, 1944 
Distribution: Ireland

Chorebus amasis (Nixon, 1945)

Nomenclature:

Dacnusa amasis Nixon, 1945

Distribution: England

Chorebus ampliator (Nees, 1834)

Nomenclature:

Alysia ampliator Nees, 1834

nigricornis (Förster, 1863, Stiphrocera)

Distribution: England, Scotland, Ireland

Chorebus anasellus (Stelfox, 1952)

Nomenclature:

Dacnusa anasella Stelfox, 1952

Distribution: Scotland, Ireland

Chorebus angelicae (Nixon, 1945)

Nomenclature:

Dacnusa angelicae Nixon, 1945

Distribution: England

Chorebus anita (Nixon, 1943)

Nomenclature:

Dacnusa anita Nixon, 1943

Distribution: England, Scotland

Chorebus aphantus (Marshall, 1895)

Nomenclature:

Dacnusa aphanta Marshall, 1895

Distribution: England, Scotland, Wales, Ireland 
Chorebus apollyon (Morley, 1924)

Nomenclature:

Dacnusa apollyon Morley, 1924

Distribution: England, Ireland

Chorebus ares (Nixon, 1944)

Nomenclature:

Dacnusa ares Nixon, 1944

Distribution: England

Chorebus armida (Nixon, 1945)

Nomenclature:

Dacnusa armida Nixon, 1945

Distribution: England, Scotland, Wales

Chorebus artemisiellus Griffiths, 1968

Distribution: England

Chorebus asramenes (Nixon, 1943)

Nomenclature:

Dacnusa asramenes Nixon, 1943

Distribution: England, Ireland

Chorebus avesta (Nixon, 1944)

Nomenclature:

Dacnusa avesta Nixon, 1944

Distribution: England, Scotland

Chorebus bathyzonus (Marshall, 1895)

Nomenclature:

Dacnusa bathyzona Marshall, 1895

ornatus (Telenga, 1935, Dacnusa)

Distribution: England, Ireland 
Chorebus bensoni (Nixon, 1943)

Nomenclature:

Dacnusa bensoni Nixon, 1943

Distribution: England, Scotland

Chorebus bres (Nixon, 1944)

Nomenclature:

Dacnusa bres Nixon, 1944

Distribution: England, Ireland

Chorebus brevicornis (Thomson, 1895)

Nomenclature:

Dacnusa brevicornis Thomson, 1895

chrysippe (Nixon, 1944, Dacnusa)

ea (Nixon, 1944, Dacnusa)

Distribution: England, Scotland, Ireland

Chorebus caelebs (Nixon, 1944)

Nomenclature:

Dacnusa caelebs Nixon, 1944

Distribution: England

Chorebus calthae Griffiths, 1967

Distribution: England

Chorebus cambricus Griffiths, 1968

Distribution: Wales

\section{Chorebus cinctus (Haliday, 1839)}

Nomenclature:

Alysia cincta Haliday, 1839

castaneiventris (Thomson, 1895, Dacnusa)

Distribution: England, Ireland 
Chorebus coxator (Thomson, 1895)

Nomenclature:

Dacnusa coxator Thomson, 1895

Distribution: England, Scotland, Wales

Chorebus crassipes (Stelfox, 1954)

Nomenclature:

Dacnusa crassipes Stelfox, 1954

Distribution: Scotland, Ireland

Chorebus credne (Nixon, 1944)

Nomenclature:

Dacnusa credne Nixon, 1944

Distribution: England, Scotland, Ireland

Chorebus crenulatus (Thomson, 1895)

Nomenclature:

Dacnusa crenulata Thomson, 1895

elegantulus (Nixon, 1937, Dacnusa)

Distribution: England, Scotland, Ireland

Chorebus crocale (Nixon, 1945)

Nomenclature:

Dacnusa crocale Nixon, 1945

Distribution: England

Chorebus cubocephalus (Telenga, 1934)

Nomenclature:

Rhizarcha cubocephala Telenga, 1934

cyclops (Nixon, 1937, Dacnusa); synonymy by Tobias (1986)

Distribution: Ireland 
Chorebus cylindricus (Telenga, 1934)

Nomenclature:

Dacnusa cylindrica Telenga, 1934

cybele (Nixon, 1937, Dacnusa); synonymy by Tobias (1986)

Distribution: England, Scotland

Chorebus cytherea (Nixon, 1937)

Nomenclature:

Dacnusa cytherea Nixon, 1937

calliope (Nixon, 1944, Dacnusa)

tesmia (Nixon, 1944, Dacnusa)

Distribution: England, Scotland, Ireland

Chorebus dagda (Nixon, 1943)

Nomenclature:

Dacnusa dagda Nixon, 1943

Distribution: England

Chorebus daimenes (Nixon, 1945)

Nomenclature:

Dacnusa daimenes Nixon, 1945

Distribution: England, Scotland

Chorebus deione (Nixon, 1944)

Nomenclature:

Dacnusa deione Nixon, 1944

Distribution: England, Scotland

Chorebus didas (Nixon, 1944)

Nomenclature:

Dacnusa didas Nixon, 1944

Distribution: England, Scotland 
Chorebus difficilis Griffiths, 1968

Distribution: England

Chorebus diremtus (Nees, 1834)

Nomenclature:

Alysia diremta Nees, 1834

diremptus (Haliday, 1839, Alysia)

Distribution: England, Ireland

Chorebus dirona (Nixon, 1945)

Nomenclature:

Dacnusa dirona Nixon, 1945

Distribution: England, Ireland

Chorebus enephes (Nixon, 1945)

Nomenclature:

Dacnusa enephes Nixon, 1945

Distribution: England

Chorebus eros (Nixon, 1937)

Nomenclature:

Dacnusa eros Nixon, 1937

Distribution: England, Scotland, Ireland

Chorebus esbelta (Nixon, 1937)

Nomenclature:

Dacnusa esbelta Nixon, 1937

Distribution: England, Scotland, Ireland

Chorebus euryale (Nixon, 1944)

Nomenclature:

Dacnusa euryale Nixon, 1944

Distribution: England 
Chorebus fallaciosae Griffiths, 1967

Distribution: England

Chorebus fallax (Nixon, 1937)

Nomenclature:

Dacnusa fallax Nixon, 1937

Distribution: England, Scotland, Ireland

Chorebus flavipes (Goureau, 1851)

Nomenclature:

Dacnusa flavipes Goureau, 1851

raissa (Nixon, 1937, Dacnusa)

Distribution: England, Scotland, Ireland

Chorebus fordi (Nixon, 1954)

Nomenclature:

Dacnusa fordi Nixon, 1954

Distribution: England

Chorebus foveolus (Haliday, 1839)

Nomenclature:

Alysia foveola Haliday, 1839

Distribution: England, Scotland, Wales, Ireland

Chorebus fuscipennis (Nixon, 1937)

Nomenclature:

Dacnusa fuscipennis Nixon, 1937

Distribution: England

Chorebus gedanensis (Ratzeburg, 1852)

Nomenclature:

Alysia gedanensis Ratzeburg, 1852

anguligena (Nixon, 1937, Dacnusa) 
Distribution: England

Chorebus glaber (Nixon, 1944)

Nomenclature:

Dacnusa glabra Nixon, 1944

Distribution: England, Ireland

Chorebus glabriculus (Thomson, 1895)

Nomenclature:

Dacnusa glabricula Thomson, 1895

cortipalpis (Nixon, 1937, Dacnusa)

Distribution: England, Scotland, Ireland

Chorebus hilaris Griffiths, 1967

Distribution: England

Chorebus hirtigena Stelfox, 1957

Distribution: Ireland

Chorebus humeralis Griffiths, 1968

Distribution: Ireland

Chorebus incertus (Goureau, 1851)

Nomenclature:

Dacnusa incerta Goureau, 1851

Distribution: England

Chorebus iphias (Nixon, 1943)

Nomenclature:

Dacnusa iphias Nixon, 1943

Distribution: England 
Chorebus kama (Nixon, 1945)

Nomenclature:

Dacnusa kama Nixon, 1945

Distribution: England

Chorebus lanigerus (Stelfox, 1957)

Nomenclature:

Gyrocampa lanigera Stelfox, 1957

Distribution: Ireland

Chorebus lar (Morley, 1924)

Nomenclature:

Dacnusa lar Morley, 1924

innanus (Nixon, 1943, Dacnusa)

Distribution: England

Chorebus larides (Nixon, 1944)

Nomenclature:

Dacnusa larides Nixon, 1944

Distribution: England, Ireland

\section{Chorebus lateralis (Haliday, 1839)}

Nomenclature:

Alysia lateralis Haliday, 1839

fuscula (Haliday, 1839, Alysia)

albicoxa (Thomson, 1895, Dacnusa)

Distribution: England, Scotland, Wales, Ireland

Chorebus leptogaster (Haliday, 1839)

Nomenclature:

Alysia leptogaster Haliday, 1839

naenia (Morley, 1924, Dacnusa)

dinae (Burghele, 1960, Dacnusa) 
Distribution: England, Ireland

Chorebus longicornis (Nees, 1811)

Nomenclature:

Bracon longicornis Nees, 1811

affinis (Nees, 1812, Bassus)

Distribution: England, Scotland, Ireland

Notes: Synonymy first established in Shenefelt (1974) but overlooked until O'Connor et al. (1999).

Chorebus lugubris (Nixon, 1937)

Nomenclature:

Dacnusa lugubris Nixon, 1937

Distribution: England, Ireland

\section{Chorebus luzulae Griffiths, 1966}

Distribution: Scotland

Notes: added by Godfray and Bland (2011)

Chorebus lychnidis Griffiths, 1967

Distribution: England

Chorebus maculigastra Shenefelt, 1974

Nomenclature:

maculata (Nixon, 1944, Dacnusa) preocc.

Distribution: England, Ireland

Chorebus merellus (Nixon, 1937)

Nomenclature:

Dacnusa merella Nixon, 1937

Distribution: England, Scotland, Ireland 
Chorebus merion (Nixon, 1945)

Nomenclature:

Dacnusa merion Nixon, 1945

Distribution: England

Chorebus miodes (Nixon, 1949)

Nomenclature:

Gyrocampa miodes Nixon, 1949

Distribution: England, Ireland

Chorebus misellus (Marshall, 1895)

Nomenclature:

Dacnusa misella Marshall, 1895

Distribution: England

Chorebus mitrus (Nixon, 1945)

Nomenclature:

Dacnusa mitra Nixon, 1945

Distribution: England, Scotland, Ireland

Chorebus nanus (Nixon, 1943)

Nomenclature:

Dacnusa nana Nixon, 1943

Distribution: England

Chorebus navicularis (Nees, 1812)

Nomenclature:

Bassus navicularis Nees, 1812

Distribution: England

Chorebus nerissus (Nixon, 1937)

Nomenclature:

Dacnusa nerissa Nixon, 1937 
Distribution: England

Chorebus nigriscaposus (Nixon, 1949)

Nomenclature:

Gyrocampa nigriscaposa Nixon, 1949

propodealis (Nixon, 1949, Gyrocampa)

Distribution: Ireland

Chorebus ninella (Nixon, 1945)

Nomenclature:

Dacnusa ninella Nixon, 1945

Distribution: England, Scotland, Ireland

Chorebus nobilis Griffiths, 1968

Distribution: England, Ireland

Chorebus nomia (Nixon, 1937)

Nomenclature:

Dacnusa nomia Nixon, 1937

Distribution: England, Ireland

Chorebus nydia (Nixon, 1937)

Nomenclature:

Dacnusa nydia Nixon, 1937

Distribution: England, Scotland, Ireland

Chorebus orbiculatae Griffiths, 1967

Distribution: England, Ireland

Chorebus ovalis (Marshall, 1896)

Nomenclature:

Dacnusa ovalis Marshall, 1896

Distribution: England, Ireland 
Chorebus parvungula (Thomson, 1895)

Nomenclature:

Dacnusa parvungula Thomson, 1895

acco (Nixon, 1943, Dacnusa)

Distribution: England, Scotland, Ireland

Chorebus perkinsi (Nixon, 1944)

Nomenclature:

Dacnusa perkinsi Nixon, 1944

Distribution: England

Chorebus petiolatus (Nees, 1834)

Nomenclature:

Alysia petiolata Nees, 1834

Distribution: England, Scotland, Ireland

Chorebus phaedra (Nixon, 1937)

Nomenclature:

Dacnusa phaedra Nixon, 1937

Distribution: England, Scotland

Chorebus pimpinellae Griffiths, 1967

Distribution: England

Chorebus pione (Nixon, 1944)

Nomenclature:

Dacnusa pione Nixon, 1944

Distribution: England

Chorebus poemyzae Griffiths, 1968

Distribution: England, Scotland 
Chorebus posticus (Haliday, 1839)

Nomenclature:

Alysia postica Haliday, 1839

gracilis (Nees, 1834, Alysia); synonymy by Achterberg (1997)

egregia (Marshall, 1895, Dacnusa)

dentatus (Tobias, 1962, Dacnusa); synonymy by Tobias (1986)

Distribution: England, Scotland, Ireland

Chorebus pulverosus (Haliday, 1839)

Nomenclature:

Alysia pulverosa Haliday, 1839

marsyas (Nixon, 1937, Dacnusa)

Distribution: England, Ireland

Chorebus punctum (Goureau, 1851)

Nomenclature:

Dacnusa punctum Goureau, 1851

Distribution: England, Scotland, Ireland

Chorebus resa (Nixon, 1937)

Nomenclature:

Dacnusa resa Nixon, 1937

Distribution: England

Chorebus rhanis (Nixon, 1943)

Nomenclature:

Dacnusa rhanis Nixon, 1943

Distribution: Scotland

Chorebus risilis (Nixon, 1949)

Nomenclature:

Gyrocampa risilis Nixon, 1949

Distribution: Ireland 
Chorebus rondanii (Giard, 1904)

Nomenclature:

Dacnusa rondanii Giard, 1904

galbus (Nixon, 1944, Dacnusa)

Distribution: England

Chorebus rotundiventris (Thomson, 1895)

Nomenclature:

Dacnusa rotundiventris Thomson, 1895

Distribution: Ireland

Chorebus rousseaui (Schulz, 1907)

Nomenclature:

Dacnusa rousseaui Schulz, 1907

Notes: The status of this taxon needs further research.

Chorebus ruficollis (Stelfox, 1957)

Nomenclature:

Gyrocampa ruficollis Stelfox, 1957

Distribution: Ireland

Chorebus rufimarginatus (Stelfox, 1954)

Nomenclature:

Dacnusa rufimarginata Stelfox, 1954

Distribution: Ireland

Chorebus scabiosae Griffiths, 1967

Distribution: England

Chorebus scabrifossa Stelfox, 1957

Distribution: Ireland 
Chorebus selene (Nixon, 1937)

Nomenclature:

Dacnusa selene Nixon, 1937

Distribution: England, Scotland

Chorebus senilis (Nees, 1812)

Nomenclature:

Bassus senilis Nees, 1812

tomentosus (Thomson, 1895, Dacnusa)

nemesis (Morley, 1924, Dacnusa)

Distribution: England, Scotland, Wales, Ireland

Chorebus serus (Nixon, 1937)

Nomenclature:

Dacnusa sera Nixon, 1937

Distribution: England

Chorebus siniffa (Nixon, 1937)

Nomenclature:

Dacnusa siniffa Nixon, 1937

Distribution: England, Ireland

Chorebus solstitialis (Stelfox, 1952)

Nomenclature:

Dacnusa solstitialis Stelfox, 1952

Distribution: England

Chorebus spenceri Griffiths, 1964

Distribution: England, Scotland

Chorebus striola Stelfox, 1957

Distribution: Ireland 
Chorebus sylvestris Griffiths, 1967

Distribution: England, Scotland, Ireland

Chorebus talaris (Haliday, 1839)

Nomenclature:

Alysia talaris Haliday, 1839

Distribution: England, Scotland, Ireland

Chorebus tamiris (Nixon, 1943)

Nomenclature:

Dacnusa tamiris Nixon, 1943

Distribution: England

Chorebus tamsi (Nixon, 1944)

Nomenclature:

Dacnusa tamsi Nixon, 1944

Distribution: England

Chorebus tanis (Nixon, 1945)

Nomenclature:

Dacnusa tanis Nixon, 1945

Distribution: England

Chorebus tenellae Griffiths, 1967

Distribution: Scotland, Ireland

Notes: added by Griffiths (1984)

Chorebus thecla (Nixon, 1943)

Nomenclature:

Dacnusa thecla Nixon, 1943

Distribution: England 
Chorebus thisbe (Nixon, 1937)

Nomenclature:

Dacnusa thisbe Nixon, 1937

Distribution: England

Chorebus thusa (Nixon, 1937)

Nomenclature:

Dacnusa thusa Nixon, 1937

Distribution: England, Ireland

Chorebus transversus (Nixon, 1954)

Nomenclature:

Dacnusa transversa Nixon, 1954

Distribution: England, Scotland, Ireland

Chorebus trilobomyzae Griffiths, 1968

Distribution: England, Scotland

Chorebus uliginosus (Haliday, 1839)

Nomenclature:

Alysia uliginosa Haliday, 1839

thienemanni (Ruschka, 1913, Gyrocampa)

Distribution: England, Ireland

Chorebus uma (Nixon, 1944)

Nomenclature:

Dacnusa uma Nixon, 1944

Distribution: England

Chorebus varuna (Nixon, 1945)

Nomenclature:

Dacnusa varuna Nixon, 1945

Distribution: England, Scotland 
Chorebus vernalis Griffiths, 1968

Distribution: England

Chorebus vitripennis Griffiths, 1968

Distribution: Scotland, Wales, Ireland

\section{Genus Coelinidea Viereck, 1913}

Nomenclature:

LEPTON Zetterstedt, 1838 preocc.

ERICOELINIUS Viereck, 1913

FISCHERASTRIOLUS Perepechayenko, 1999

Notes: There is no recent treatment of this genus; generic synonymy follows Achterberg (2014).

\section{Coelinidea elegans (Curtis, 1829)}

Nomenclature:

Chaenon elegans Curtis, 1829

brevicornis (Curtis, 1829, Chaenon)

cingulatus (Curtis, 1829, Chaenon)

rufinotatus (Curtis, 1829, Chaenon)

similis (Curtis, 1829, Chaenon)

Distribution: England, Ireland

\section{Coelinidea fuliginosus (Curtis, 1829)}

Nomenclature:

Chaenon fuliginosus Curtis, 1829

Distribution: England

\section{Coelinidea gracilis (Curtis, 1829)}

Nomenclature:

Chaenon gracilis Curtis, 1829

attenuator (Zetterstedt, 1838, Lepton)

Distribution: England, Scotland, Ireland 
Coelinidea niger (Nees, 1811)

Nomenclature:

Stephanus niger Nees, 1811

affinis (Curtis, 1829, Chaenon)

nigricans (Westwood, 1835, Chaenon)

olivieri (Guérin-Ménéville, 1842, Alysia)

Distribution: England, Ireland

Coelinidea obscurus (Curtis, 1829)

Nomenclature:

Chaenon obscurus Curtis, 1829

Distribution: England

Coelinidea podagricus (Haliday, 1839)

Nomenclature:

Alysia podagrica Haliday, 1839

Distribution: Ireland

Coelinidea ruficollis (Herrich-Schäffer, 1838)

Nomenclature:

Coelinius ruficollis Herrich-Schäffer, 1838

procerus (Haliday, 1839, Alysia)

Distribution: England

Coelinidea viduus (Curtis, 1829)

Nomenclature:

Chaenon viduus Curtis, 1829

ater (Curtis, 1837, Chaenon) nom. nud.

Distribution: England, Ireland

Genus Coelinius Nees, 1818

Nomenclature:

CHAENON Curtis, 1829 
COPISURA Schiødte, 1837

COPIDURA Förster,1862

Notes: Griffiths (1964) proposed that Polemochartus and Coelinidea should be included as subgenera of Coelinius. Wharton and Austin (1991), Wharton (1994) and Kula (2008) supported the concept of an enlarged Coelinius but, pointing to intermediate taxa in the Oriental Region, argued against the retention of subgenera. Achterberg (2014) retains the three genera which is current European usage and is followed here pending a modern review of the group.

\section{Coelinius parvulus (Nees, 1811)}

Nomenclature:

Chaenon parvulus Nees, 1811

?cultriformis (Latreille, 1802, Ichneumon)

circulator (Gravenhorst, 1807, Ichneumon) preocc.

anceps (Curtis, 1829, Chaenon)

rimator (Schiødte, 1837, Copisura)

bicarinatus Herrich-Schäffer, 1838

flexuosus Herrich-Schäffer, 1838

bicolor Maréchal, 1938

Distribution: England, Scotland, Ireland, Isle of Man

\section{Genus Coloneura Förster, 1863}

Nomenclature:

ISOMERISTA Förster, 1863

TRISISA Förster, 1863

MERITES Nixon, 1943

PRIAPSIS Nixon, 1943

\section{Coloneura dice (Nixon, 1943)}

Nomenclature:

Priapsis dice Nixon, 1943

Distribution: England 
Coloneura stylata Förster, 1863

Nomenclature:

exilis (Förster, 1863, Trisisa)

oligomera (Förster, 1863, Isomerista)

taras (Nixon, 1943, Merites)

Distribution: England, Ireland

Genus Dacnusa Haliday, 1833

Nomenclature:

AGONIA Förster, 1863

APHANTA Förster, 1863

BRACHYSTROPHA Förster, 1863

LIPOSCIA Förster, 1863

PACHYSEMA Förster, 1863

RHIZARCHA Förster, 1863

TANYSTROPHA Förster, 1863

RADIOLARIA Provancher, 1886

COLONEURELLA van Achterberg, 1976

Dacnusa abdita Haliday, 1838

Nomenclature:

incidens Thomson, 1895

lepida Marshall, 1896

Distribution: England, Scotland, Wales, Ireland

Dacnusa adducta (Haliday, 1839)

Nomenclature:

Alysia adducta Haliday, 1839

abducta misspelling

Distribution: England, Scotland, Ireland 
Notes: Based on its unusual venation, adducta is often placed in the monotypic genus Agonia; Griffiths' (Griffiths 1964) argument that it is a derived species of Dacnusa is followed here.

\section{Dacnusa alticeps Nixon, 1937}

Distribution: England, Scotland

Dacnusa aquilegiae Marshall, 1896

Distribution: England, Scotland

Dacnusa areolaris (Nees, 1811)

Nomenclature:

Bracon areolaris Nees, 1811

lysias Goureau, 1851

Distribution: England, Scotland, Wales, Ireland

\section{Dacnusa astarte (Nixon, 1948)}

Nomenclature:

Rhizarcha astarte Nixon, 1948

Distribution: England

Dacnusa aterrima Thomson, 1895

Distribution: England

\section{Dacnusa confinis Ruthe, 1859}

Nomenclature:

minuta (Curtis, 1826, Alysia)

Distribution: England, Wales, Ireland

Notes: Alysia minuta was listed as a species of Alysia by Shenefelt (1974) and Huddleston (1978) although a junior homonym of Alysia minuta Nees, 1812; Wharton (1986) showed it to belong to Dacnusa and van Achterberg (in litt.) concluded that it is conspecific with confinis. 
Dacnusa delphinii Griffiths, 1967

Distribution: England

Dacnusa discolor (Förster, 1863)

Nomenclature:

Liposcia discolor Förster, 1863

cercides (Nixon, 1954, Pachysema)

Distribution: England, Scotland, Ireland

Dacnusa dryas (Nixon, 1948)

Nomenclature:

Rhizarcha dryas Nixon, 1948

Distribution: England

Dacnusa ergeteles (Nixon, 1954)

Nomenclature:

Pachysema ergeteles Nixon, 1954

Distribution: Ireland

Dacnusa euphrasiella Griffiths, 1984

Distribution: Ireland

Notes: added by Griffiths (1984)

Dacnusa evadne Nixon, 1937

Distribution: England, Scotland, Ireland

Dacnusa faeroeensis (Roman, 1917)

Nomenclature:

Rhizarcha faeroeensis Roman, 1917

lestes Nixon, 1937

Distribution: England, Scotland, Wales, Ireland 
Dacnusa fasciata Stelfox, 1954

Distribution: Ireland

Dacnusa hospita (Förster, 1863)

Nomenclature:

Aphanta hospita Förster, 1863

Distribution: England, Scotland, Ireland

Dacnusa laeta (Nixon, 1954)

Nomenclature:

Pachysema laeta Nixon, 1954

Distribution: England, Scotland, Ireland

Dacnusa laevipectus Thomson, 1895

Nomenclature:

nox (Morley, 1924, Rhizarcha)

Distribution: England, Scotland, Wales, Ireland

Dacnusa lissos (Nixon, 1954)

Nomenclature:

Pachysema lissos Nixon, 1954

Distribution: England

Dacnusa longiradialis Nixon, 1937

Distribution: England, Scotland, Ireland

Dacnusa lugens (Haliday, 1839)

Nomenclature:

Alysia lugens Haliday, 1839

Distribution: England, Ireland 
Dacnusa macrospila (Haliday, 1839)

Nomenclature:

Alysia macrospila Haliday, 1839

Distribution: England, Scotland, Ireland

Dacnusa maculipes Thomson, 1895

Distribution: England, Scotland, Wales, Ireland

Dacnusa maxima (Fischer, 1961)

Nomenclature:

Pachysema maxima Fischer, 1961

Distribution: Wales

Dacnusa melicerta (Nixon, 1954)

Nomenclature:

Pachysema melicerta Nixon, 1954

fumipes Tobias, 1998

Distribution: England, Scotland, Ireland

Dacnusa merope (Nixon, 1948)

Nomenclature:

Rhizarcha merope Nixon, 1948

Distribution: England

Dacnusa metula (Nixon, 1954)

Nomenclature:

Pachysema metula Nixon, 1954

Distribution: England, Scotland, Ireland

Dacnusa monticola (Förster, 1863)

Nomenclature:

Brachystropha monticola Förster, 1863

mutia (Nixon, 1948, Rhizarcha) 
coracina Stelfox, 1957

Distribution: Ireland

Dacnusa nigrella Griffiths, 1967

Distribution: Scotland

Notes: added by Godfray and Bland (2011)

Dacnusa nigropygmaea Stelfox, 1954

Distribution: Ireland

Dacnusa obesa Stelfox, 1954

Distribution: Scotland, Ireland

Dacnusa ocyroe Nixon, 1937

Distribution: England, Scotland, Wales, Ireland

Dacnusa plantaginis Griffiths, 1967

Nomenclature:

discolor misident.

Distribution: England, Scotland, Wales, Ireland

Dacnusa pubescens (Curtis, 1826)

Nomenclature:

Alysia pubescens Curtis, 1826

exserens (Nees, 1834, Alysia)

Distribution: England, Scotland, Ireland

Dacnusa sibirica Telenga, 1934

Distribution: England, Scotland, Wales, Ireland

Dacnusa comis (Nixon, 1954)

Nomenclature:

Pachysema comis Nixon, 1954 
Dacnusa soma (Nixon, 1948)

Nomenclature:

Rhizarcha soma Nixon, 1948

Distribution: Scotland, Ireland

Dacnusa stramineipes (Haliday, 1839)

Nomenclature:

Alysia stramineipes Haliday, 1839

haemorrhoa (Förster, 1863, Tanystropha)

longicauda Thomson, 1895

Distribution: England, Scotland, Ireland

Notes: Dacnusa longicauda was synonymised by Nixon (1937) although this was overlooked by Huddleston (1978).

Dacnusa tarsalis Thomson, 1895

Nomenclature:

nitetis (Nixon, 1948, Rhizarcha)

Distribution: England, Scotland, Wales, Ireland

Dacnusa temula (Haliday, 1839)

Nomenclature:

Alysia temula Haliday, 1839

Distribution: England, Scotland, Ireland

Dacnusa veronicae Griffiths, 1967

Distribution: England, Scotland

Genus Epimicta Förster, 1863

Epimicta marginalis (Haliday, 1839)

Nomenclature:

Alysia marginalis Haliday, 1839

Distribution: England, Ireland 


\section{Genus Exotela Förster, 1863}

Nomenclature:

MESORA Förster, 1863

TOXELEA Nixon, 1943

ANTRUSA Nixon, 1943

Notes: Griffiths' (Griffiths 1964, Griffiths 1967a, Griffiths 1967b, Griffiths 1967c, Griffiths 1968a, Griffiths 1968b) concept of Exotela is followed here; the most plesiomorphic species (flavicoxa, interstitialis, melanocera and vaenia) are often placed in Antrusa (e.g. Achterberg 2014).

\section{Exotela cyclogaster Förster, 1863}

\section{Nomenclature:}

bellina (Nixon, 1937, Dacnusa)

umbellina (Nixon, 1954, Toxelea)

sonchina Griffiths, 1967

Distribution: England, Scotland, Wales, Ireland

Notes: Griffiths (1967c) divided cyclogaster into three subspecies, nominate cyclogaster, umbellina and sonchina. Tobias (1986) later treated these as separate species although Godfray (1984) argued that umbellina and cyclogaster are host race variants. Pending further research they are treated here as a single taxon.

\section{Exotela dives (Nixon, 1954)}

Nomenclature:

Toxelea dives Nixon, 1954

Distribution: Ireland

\section{Exotela flavicoxa (Thomson, 1895)}

Nomenclature:

Dacnusa flavicoxa Thomson, 1895

Distribution: England, Scotland, Ireland

\section{Exotela gilvipes (Haliday, 1839)}

\section{Nomenclature:}

Alysia gilvipes Haliday, 1839 
albilabris (Thomson, 1895, Dacnusa)

Distribution: England, Scotland, Ireland

Exotela hera (Nixon, 1937)

Nomenclature:

Dacnusa hera Nixon, 1937

Distribution: England, Scotland, Isle of Man

Exotela interstitialis (Thomson, 1895)

Nomenclature:

Dacnusa interstitialis Thomson, 1895

mamertes (Nixon, 1943, Dacnusa)

Notes: Placed in Chorebus by Tobias and Jakimavicius (1973) and in Antrusa by Papp (2007); needs further research.

\section{Exotela lonicerae Griffiths, 1967}

Distribution: England, Scotland

Exotela melanocera (Thomson, 1895)

Nomenclature:

Dacnusa melanocera Thomson, 1895

persimilis (Nixon, 1954, Antrusa)

Distribution: England, Ireland

Exotela phryne (Nixon, 1954)

Nomenclature:

Toxelea phryne Nixon, 1954

Distribution: England, Scotland

Exotela spinifer (Nixon, 1954)

Nomenclature:

Toxelea spinifer Nixon, 1954

Distribution: England, Scotland, Ireland 
Exotela sulcata (Tobias, 1962)

Nomenclature:

Pachysema sulcata Tobias, 1962

Distribution: England, Scotland, Ireland

Exotela vaenia (Nixon, 1954)

Nomenclature:

Antrusa vaenia Nixon, 1954

Distribution: England

Exotela viciae Griffiths, 1984

Distribution: England

Notes: added by Griffiths (1984)

Genus Laotris Nixon, 1943

Laotris striatula (Haliday, 1839)

Nomenclature:

Alysia striatula Haliday, 1839

Distribution: England, Scotland, Ireland

Genus Polemochartus Nees, 1818

Polemochartus liparae (Giraud, 1863)

Nomenclature:

Polemon liparae Giraud, 1863

Distribution: England

Polemochartus melas (Giraud, 1863)

Nomenclature:

Polemon melas Giraud, 1863

Distribution: England

Notes: added by Shaw and Jennings (2008) 
Genus Protodacnusa Griffiths, 1964

Protodacnusa litoralis Griffiths, 1964

Distribution: Ireland

Protodacnusa tristis (Nees, 1834)

Nomenclature:

Alysia tristis Nees, 1834

ampliator (Haliday, 1839, Alysia) preocc.

longistigma (Telenga, 1935, Dacnusa)

Distribution: England, Ireland

Genus Sarops Nixon, 1942

Sarops rea Nixon, 1942

Distribution: England

Genus Synelix Förster, 1863

Nomenclature:

ECTILIS Nixon, 1943

Synelix semirugosa (Haliday, 1839)

Nomenclature:

Alysia semirugosa Haliday, 1839

agnata Förster, 1863

amaurosomae (Telenga, 1935, Dacnusa)

Distribution: England, Scotland, Wales, Ireland

Genus Tates Nixon, 1943

Tates heterocera (Thomson, 1895)

Nomenclature:

Dacnusa heterocera Thomson, 1895

Distribution: England, Scotland 
Genus Trachionus Haliday, 1833

Subgenus Trachionus Haliday, 1833

Nomenclature:

AENONE Curtis, 1837 preocc.

AENONE Haliday, 1838 preocc., nom. nud.

OENONE Haliday, 1839 preocc.

SYMPHYA Förster, 1863

ANARMUS Ruthe, 1882

Notes: Generic synonymy follows Achterberg (1997).

Trachionus (Trachionus) mandibularis (Nees, 1816)

Nomenclature:

Sigalphus mandibularis Nees, 1816

Distribution: England, Wales, Ireland

Subgenus Planiricus Perepechayenko, 2000

Trachionus (Planiricus) hians (Nees, 1816)

Nomenclature:

Sigalphus hians Nees, 1816

Distribution: England, Scotland, Ireland

Trachionus (Planiricus) ringens (Haliday, 1839)

Nomenclature:

Alysia ringens Haliday, 1839

Distribution: England, Scotland, Ireland 


\section{Aphidiinae}

\section{Subfamily Aphidiinae Haliday, 1833}

Notes: In the older literature (and some very recent literature) often treated as a separate family (i.e. Aphidiidae) within Ichneumonoidea. Some distribution data from Mackauer (1961), Starý (1978) and Baker (2013).

Tribe Aphidiini Haliday, 1833

\section{Genus Adialytus Förster, 1863}

\section{Adialytus ambiguus (Haliday, 1834)}

Nomenclature:

$$
\begin{aligned}
& \text { Aphidius ambiguus Haliday, } 1834 \\
& \text { diminuens (Nees, 1834, Aphidius) } \\
& \text { ?exiguus (Haliday, 1834, Aphidius) } \\
& \text { delhiensis (Subba Rao \& Sharma, 1960, Aphidius) } \\
& \text { arvicola (Starý, 1961, Lysiphlebus) } \\
& \text { mackaueri (Starý, 1961, Lysiphlebus) } \\
& \text { crocinus (Mackauer, 1962, Lysiphlebus) }
\end{aligned}
$$

Distribution: Scotland, Wales

Notes: The type of exiguus is lost (Achterberg 1997).

\section{Adialytus salicaphis (Fitch, 1855)}

\section{Nomenclature:}

$$
\begin{aligned}
& \text { Trioxys salicaphis Fitch, } 1855 \\
& \text { populaphis (Fitch, 1855, Trioxys) } \\
& \text { tenuis Förster, } 1863 \\
& \text { salicaphidis (Ashmead, 1889, Lipolexis) } \\
& \text { laticephalus (Telenga, 1953, Aphidius) }
\end{aligned}
$$

Distribution: England, Wales

Notes: added by Baker and Broad (2009) 


\section{Genus Aphidius Nees, 1818}

\section{Nomenclature:}

INCUBUS Schrank, 1802 nom. ob.

THERACMION Holmgren, 1872

EUAPHIDIUS Mackauer, 1961

Notes: doubtfully placed species of Aphidius:

[constrictus (Nees, 1811, Bracon)] Included as a British species by Lyle (1933) on the basis of Szépligeti's (Szépligeti 1904) Palaearctic catalogue; the type is lost and the species has not been interpreted by recent authors.

[dimidiatus Curtis, 1831]

[pallidinotus Haliday, 1834 nom. nud.]

[pseudoplatanus Curtis, 1837; constrictus misident.] There have been no references to this species other than catalogue listings (Smith 1853, Marshall 1872). Although proposed (Curtis 1837) as a name for the species called Aphidius constrictus by Haliday, it has not been interpreted by recent authors.

[viminalis Haliday, 1834 nom. nud.]

\section{Aphidius absinthii Marshall, 1896}

Distribution: England

Notes: Treated as a valid species of Aphidius by Rakhshani et al. (2011) rather than a synonym of asteris.

\section{Aphidius aquilus Mackauer, 1961}

Nomenclature:

sicarius Mackauer, 1961

callipterinellae (Takada, 1966, Lysaphidus)

Distribution: England, Wales

\section{Aphidius asteris Haliday, 1834}

Nomenclature:

melanocephalus (Nees, 1811, Bracon)

lutescens Haliday, 1834

commodus Gahan, 1926 
artemisiae Ivanov, 1927

Distribution: England, Wales

\section{Aphidius avenae Haliday, 1834}

Nomenclature:

picipes (Nees, 1811, Bracon) suppressed

crithmi Marshall, 1896

granarius Marshall, 1896

pascuorum Marshall, 1896

hungaricus (Györfi, 1958, Lysiphlebus)

caraganae Starý, 1963

Distribution: England, Wales, Ireland

\section{Aphidius cingulatus Ruthe, 1859}

Nomenclature:

arcticus (Holmgren, 1872, Theracmion)

gregarius Marshall, 1872

lachni Ashmead, 1889

pterocommae Ashmead, 1889

pterocommae Marshall, 1896 preocc.

luzhetzki Telenga, 1958

Distribution: England, Wales

\section{Aphidius colemani Viereck, 1912}

Nomenclature:

huebrichi Brèthes, 1913

platensis Brèthes, 1913

porteri Brèthes, 1915

aphidiphilus Benoit, 1955

leroyi Benoit, 1955

transcaspicus Telenga, 1958

Notes: \#Introduced into greenhouses for biocontrol (Starý 1975). 
Aphidius eadyi Starý, Gonzalez \& Hall, 1980

Nomenclature:

urticae misident.

Distribution: England

Notes: added by Müller et al. (1999)

Aphidius eglanteriae Haliday, 1834

Aphidius ervi Haliday, 1834

Nomenclature:

infirmus (Nees, 1811, Bracon)

ulmi Marshall, 1896

medicaginis Marshall, 1898

fumipennis Györfi, 1958

nigrescens Mackauer, 1962

mirotarsi Starý, 1963

Distribution: England, Wales, Ireland

Aphidius fumatus Haliday, 1834

Aphidius funebris Mackauer, 1961

Nomenclature:

cirsii Ivanov, 1925 preocc.

bispinosus Telenga, 1958

eriophori Mackauer, 1967

Distribution: England, Wales

Notes: added by Pennacchio (1989)

Aphidius hortensis Marshall, 1896

Nomenclature:

berberidis Smith, 1944

Distribution: England, Wales 
Aphidius matricariae Haliday, 1834

Nomenclature:

arundinis Haliday, 1834

cirsii Haliday, 1834 preocc.

phorodontis Ashmead, 1889

chrysanthemi Marshall, 1896

lychnidis Marshall, 1896

polygoni Marshall, 1896

affinis Quilis, 1931

baudysi Quilis, 1931

discrytus Quilis, 1931

merceti Quilis, 1931

obscuriformis Quilis, 1931

valentinus Quilis, 1931

renominatus Hincks, 1943

nigriteleus Smith, 1944

Distribution: England, Wales, Ireland

Aphidius microlophii Pennacchio \& Tremblay, 1987

Distribution: England, Wales

Notes: Added by Pennacchio (1989); also recorded by Müller et al. (1999).

Aphidius rhopalosiphi de Stefani-Perez, 1902

Nomenclature:

equiseticola Starý, 1963

poacearum Starý, 1963

Distribution: England

\section{Aphidius ribis Haliday, 1834}

Nomenclature:

ribaphidis (Ashmead, 1889, Lysiphlebus)

scabiosae Marshall, 1896

ribis Ashmead, 1898 preocc. 
Distribution: England

\section{Aphidius rosae Haliday, 1833}

\section{Nomenclature:}

aphidum (Linnaeus, 1758, Ichneumon) nom. ob.

aphidator (Thunberg, 1824, Ichneumon) nom. ob.

rosae Curtis, 1831 nom. nud.

protaeus Wesmael, 1835

rosarum Nees, 1834

xanthostoma Bouché, 1834

protaeus Wesmael, 1835

cancellatus Buckton, 1876

Distribution: England, Wales, Ireland

\section{Aphidius rubi Starý, 1962}

Distribution: England

Notes: Added by Müller et al. (1999); Belshaw (appendix in Müller et al. 1999) demonstrates that this species should be treated as separate from urticae.

\section{Aphidius salicis Haliday, 1834}

Nomenclature:

restrictus Nees, 1834

duodecimarticulatus Ratzeburg, 1852

dauci Marshall, 1896

Distribution: England, Wales

\section{Aphidius setiger (Mackauer, 1961)}

Nomenclature:

Euaphidius setiger Mackauer, 1961

aceri Ivanov, 1925

Distribution: Wales

Notes: added by Baker and Broad (2009) 
Aphidius sonchi Marshall, 1896

Distribution: England, Wales

\section{Aphidius tanacetarius Mackauer, 1962}

Nomenclature:

tanaceti Curtis, 1837 nom. nud.

tanaceticola Starý, 1963

Notes: Belokobylskij et al. (2003) treat tanacetarius as the valid name for tanaceti, which is a nomen nudum. This species has not been listed as British since its original description (Curtis 1837) and Smith's (Smith 1853) catalogue. Pungerl (1986) treated tanacetarius as a valid species but did not see any British or Irish material.

\section{Aphidius urticae Haliday, 1834}

\section{Nomenclature:}

euphorbiae Marshall, 1896

longulus Marshall, 1896

lonicerae Marshall, 1896

silenes Marshall, 1896

goidanichi Quilis, 1932

ivanovae Telenga, 1958

rubi Starý, 1962

silvaticus Starý, 1962

aulacorthi Starý, 1963

Distribution: England

\section{Aphidius uzbekistanicus Luzhetski, 1960}

\section{Nomenclature:}

urticae misident.; Powell (1982)

?beltrani Quilis, 1931

?indivisus Quilis, 1931

?macropterus Quilis, 1931

?pailloti Quilis, 1931

impressus Mackauer, 1965

Distribution: England 
Notes: Aphidius beltrani is a possible senior synonym of uzbekistanicus (Starý 1973).

\section{Genus Betuloxys Mackauer, 1960}

\section{Betuloxys compressicornis (Ruthe, 1859)}

\section{Nomenclature:}

Trioxys compressicornis Ruthe, 1859

testaceus (Stelfox, 1948, Trioxys)

Distribution: England, Wales, Ireland

\section{Genus Binodoxys Mackauer, 1960}

\section{Nomenclature:}

MISAPHIDUS Rondani, 1848 nom. ob.

Notes: In Belokobylskij et al. (2003), van Achterberg listed these species under the genus name Misaphidus but, according to van Achterberg (pers. comm.), Misaphidus Rondani, 1848 s.l. (type-species: Misaphidus crudelis Rondani, 1848 (= Aphidius centaureae Haliday, 1833)) is a nomen oblitum according to Article 23.9 of ICZN.

\section{Binodoxys acalephae (Marshall, 1896)}

Nomenclature:

Aphidius acalephae Marshall, 1896

rietscheli (Mackauer, 1959, Trioxys)

urticae (Mackauer, 1959, Trioxys)

Distribution: England

\section{Binodoxys angelicae (Haliday, 1833)}

Nomenclature:

Aphidius angelicae Haliday, 1833

placidus (Gautier, 1922, Trioxys)

boscai (Quilis, 1931, Trioxys)

fumariae (Quilis, 1931, Trioxys)

granatensis (Quilis, 1931, Trioxys)

obscuriformis (Quilis, 1931, Trioxys)

amoplanus (Quilis, 1934, Trioxys) 
mediterraneus (Mackauer, 1960, Trioxys)

wollastonii (Cabrera, 1962, Trioxys) nom. nud.

sikkimensis (Raychaudhuri, Samanta, Pramanik, Tamili \& Sarkar, 1990, Trioxys)

Distribution: Wales

\section{Binodoxys brevicornis (Haliday, 1833)}

Nomenclature:

Aphidius brevicornis Haliday, 1833

minutus (Haliday, 1833, Aphidius)

Distribution: Wales, Ireland

\section{Binodoxys centaureae (Haliday, 1833)}

Nomenclature:

Aphidius centaureae Haliday, 1833

crudelis (Rondani, 1848, Misaphidus)

orientalis (Starý \& Schlinger, 1967, Trioxys)

uroleucon Takada \& Rishi, 1980

Distribution: England, Scotland, Wales, Ireland

Binodoxys heraclei (Haliday, 1833)

Nomenclature:

Aphidius heraclei Haliday, 1833

obsoletus (Wesmael, 1835, Aphidius)

variegator (Szépligeti, 1898, Trioxys)

Distribution: Wales

\section{Binodoxys letifer (Haliday, 1833)}

Nomenclature:

Aphidius letifer Haliday, 1833

Distribution: Ireland 


\section{Genus Diaeretellus Starý, 1960}

Diaeretellus ephippium (Haliday, 1834)

Nomenclature:

Aphidius ephippium Haliday, 1834

Distribution: England, Ireland

Genus Diaeretiella Starý, 1960

Diaeretiella rapae (McIntosh, 1855)

Nomenclature:

Aphidius rapae McIntosh, 1855

vulgaris (Bouché, 1834, Aphidius)

rapae (Curtis, 1860, Aphidius) preocc.

chenopodii (Förster, 1867, Diaeretus) nom. nud.

halticae (Rondani, 1877, Misaphidus)

piceus (Cresson, 1879, Trioxys)

chenopodiaphidis (Ashmead, 1889, Lipolexis)

ferruginipes (Ashmead, 1890, Diaeretus) nom. nud.

brassicae (Marshall, 1896, Aphidius)

Distribution: England, Wales

Genus Diaeretus Förster, 1863

Diaeretus leucopterus (Haliday, 1834)

Nomenclature:

Aphidius leucopterus Haliday, 1834

exspectatus (Gautier \& Bonnamour, 1936, Aphidius)

Distribution: England, Wales

Genus Harkeria Cameron, 1900

Nomenclature:

PARAMONOCTONUS Starý, 1959 
Harkeria rufa Cameron, 1900

Distribution: England

Notes: Distribution from Achterberg (1989), who elevated Harkeria from a subgenus of Monoctonus.

\section{Genus Lipolexis Förster, 1863}

Nomenclature:

GYNOCRYPTUS Quilis, 1931

\section{Lipolexis gracilis Förster, 1863}

Nomenclature:

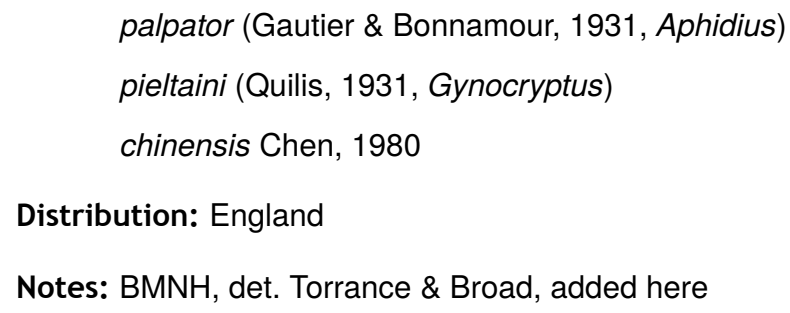

Notes: BMNH, det. Torrance \& Broad, added here

Distribution: England

Genus Lysaphidus Smith, 1944

Lysaphidus schimitscheki Starý, 1960

Distribution: England

Genus Lysaphidus Förster, 1863

Subgenus Lysiphlebus Förster, 1863

Nomenclature:

PLATYCYPHUS Mackauer, 1960

Lysiphlebus (Lysiphlebus) dissolutus (Nees, 1811)

Nomenclature:

Bracon dissolutus Nees, 1811

macrocornis Mackauer, 1960

Distribution: England 
Subgenus Phlebus Starý, 1975

Nomenclature:

APHIDARIA Provancher, 1888 preocc.

\section{Lysiphlebus (Phlebus) confusus Tremblay \& Eady, 1978}

Distribution: England, Wales

Notes: Added here on the basis of specimens in BMNH, identified by R.D. Eady as ambiguus sensu Mackauer and Starý nec Haliday, later described by Tremblay and Eady (1978) as a separate species, confusus, the true ambiguus belonging in Adialytus.

\section{Lysiphlebus (Phlebus) fabarum (Marshall, 1896)}

\section{Nomenclature:}

Aphidius fabarum Marshall, 1896

aphidiperda (Rondani, 1877, Misaphidus)

monilicornis (Thomson, 1895, Aphidius)

cardui (Marshall, 1896, Aphidius)

aurantii (Pierantoni, 1907, Aphidius)

polygoni (Ivanov, 1927, Aphidius) preocc.

gomezi (Quilis, 1930, Aphidius)

janinii (Quilis, 1930, Aphidius)

inermis Quilis, 1931

innovatus Quilis, 1931

moroderi Quilis, 1931

ivanovi Mackauer, 1967

Distribution: England, Wales

Genus Monoctonia Starý, 1962

\section{Monoctonia vesicarii Tremblay, 1991}

Distribution: England

Notes: added by Rakhshani et al. (2015) 
Genus Monoctonus Haliday, 1833

Subgenus Falciconus Mackauer, 1961

Monoctonus (Falciconus) pseudoplatani (Marshall, 1896)

Nomenclature:

Aphidius pseudoplatani Marshall, 1896

Distribution: England, Wales

Subgenus Monoctonus Haliday, 1833

Monoctonus (Monoctonus) caricis (Haliday, 1833)

Nomenclature:

Aphidius caricis Haliday, 1833

Distribution: England, Wales

Monoctonus (Monoctonus) cerasi (Marshall, 1896)

Nomenclature:

Aphidius cerasi Marshall, 1896

Distribution: England

Monoctonus (Monoctonus) crepidis (Haliday, 1834)

Nomenclature:

Aphidius crepidis Haliday, 1834

tuberculatus (Wesmael, 1835, Aphidius)

paludum Marshall, 1896

Distribution: England, Wales

Monoctonus (Monoctonus) ligustri van Achterberg, 1989

Distribution: England, Wales

Notes: added by Achterberg (1989) 
Monoctonus (Monoctonus) nervosus (Haliday, 1833)

Nomenclature:

Aphidius nervosus Haliday, 1833

paulensis (Ashmead, 1902, Aphidius)

secundus Viereck, 1915

biroi Györfi, 1958

breviantennalis Starý, 1959

\section{Genus Paralipsis Förster, 1863}

Nomenclature:

Myrmecobosca Maneval, 1940

\section{Paralipsis enervis (Nees, 1834)}

Nomenclature:

Aphidius enervis Nees, 1834

mandibularis (Maneval, 1940, Myrmecobosca)

linnei (Hincks, 1949, Myrmecobosca)

Distribution: England, Wales

\section{Genus Pauesia Quilis Pérez, 1931}

\section{Subgenus Paraphidius Starý,1958}

Notes: species of Pauesia (Paraphidius) excluded from the British and Irish list:

[silvestris (Starý, 1960, Paraphidius)] added by Enobakhare (2001) but we do not know where Enobokhare's material is deposited and the identification needs to be checked.

Pauesia (Paraphidius) abietis (Marshall, 1896)

Nomenclature:

Aphidius abietis Marshall, 1896

Distribution: England

Pauesia (Paraphidius) cupressobii (Starý, 1960)

Nomenclature:

Paraphidius cupressobii Starý, 1960 
Distribution: Scotland

Notes: BMNH, det. Gärdenfors, added here

Pauesia (Paraphidius) juniperorum (Starý, 1960)

Nomenclature:

Paraphidius juniperorum Starý, 1960

Distribution: England, Scotland

Pauesia (Paraphidius) pini (Haliday, 1834)

Nomenclature:

Aphidius pini Haliday, 1834

planistipes (Nees,1834, Aphidius)

varia (Nees, 1834, Aphidius)

panzerii (Rondani, 1848, Aphidius)

lachnivorus (Ashmead, 1906, Aphidius)

Distribution: England, Scotland, Wales, Ireland

Subgenus Pauesia Quilis Pérez, 1931

Pauesia (Pauesia) infulata (Haliday, 1834)

Nomenclature:

Aphidius infulatus Haliday, 1834

albiflagellaris (Starý, 1960, Paraphidius)

Distribution: Scotland

Pauesia (Pauesia) laricis (Haliday, 1834)

Nomenclature:

Aphidius laricis Haliday, 1834

Distribution: Wales

Pauesia (Pauesia) picta (Haliday, 1834)

Nomenclature:

Aphidius pictus Haliday, 1834 
Distribution: Wales

Pauesia (Pauesia) unilachni (Gahan, 1926)

Nomenclature:

Aphidius unilachni Gahan, 1926

albuferensis Quilis, 1931

praevisus (Gautier \& Bonnamour, 1936, Aphidius)

basilewskyi (Benoit, 1955, Trioxys)

Distribution: England, Wales

Notes: added by Belshaw and Quicke (1997)

Genus Trioxys Haliday, 1833

Subgenus Pectoxys Mackauer, 1960

Trioxys (Pectoxys) macroceratus Mackauer, 1960

Subgenus Trioxys Haliday, 1833

Nomenclature:

APHIDILEO Rondani, 1877

NEVROPENES Provancher, 1886

BIOXYS Starý \& Schlinger, 1967

Trioxys (Trioxys) auctus (Haliday, 1833)

Nomenclature:

Aphidius auctus Haliday, 1833

flaviceps Szépligeti, 1898

Distribution: Ireland

Trioxys (Trioxys) betulae Marshall, 1896

Nomenclature:

solani Ivanov, 1925

hincksi Mackauer, 1960

Distribution: England, Wales 
Trioxys (Trioxys) cirsii (Curtis, 1831)

Nomenclature:

Aphidius cirsii Curtis, 1831

aceris (Haliday, 1833, Aphidius)

Distribution: England, Wales, Ireland

Trioxys (Trioxys) curvicaudus Mackauer, 1967

Distribution: England, Wales

Notes: added by Starý (1978)

Trioxys (Trioxys) falcatus Mackauer, 1959

Distribution: Wales

Notes: added by Baker and Broad (2009)

Trioxys (Trioxys) ibis Mackauer, 1961

Trioxys (Trioxys) pallidus (Haliday, 1833)

Nomenclature:

Aphidius pallidus Haliday, 1833

resolutus (Nees, 1834, Aphidius)

callipteri (Marshall, 1896, Aphidius)

pulcher Gautier \& Bonnamour, 1924

Distribution: England, Wales

Trioxys (Trioxys) tenuicaudus Starý, 1978

Distribution: Wales

Notes: added by Baker and Broad (2013) 
Tribe Ephedrini Mackauer, 1961

Genus Ephedrus Haliday, 1833

Notes: Synonymy and distribution data from Gärdenfors (1986).

Subgenus Breviephedrus Gärdenfors, 1986

Ephedrus (Breviephedrus) brevis Stelfox, 1941

Nomenclature:

picticornis Stelfox, 1941

niger Stelfox, 1941 nom. nud.

Distribution: England, Scotland, Ireland

Subgenus Ephedrus Haliday, 1833

Nomenclature:

ELASSUS Wesmael, 1835

Ephedrus (Ephedrus) cerasicola Starý, 1962

Distribution: England

Notes: added by Van Veen et al. (2008)

Ephedrus (Ephedrus) helleni Mackauer, 1968

Nomenclature:

salicicola Takada, 1968

Distribution: Scotland

Notes: added by Baker and Broad (2013)

Ephedrus (Ephedrus) lacertosus (Haliday, 1833)

Nomenclature:

Aphidius lacertosus Haliday, 1833

muesebecki Smith, 1944

Distribution: England, Scotland, Ireland 
Ephedrus (Ephedrus) laevicollis (Thomson, 1895)

Nomenclature:

Aphidius laevicollis Thomson, 1895

brevicornis (Nees, 1834, Aphidius) preocc.

minor Stelfox, 1941

salicicola Takada, 1968

Distribution: Wales, Ireland

Ephedrus (Ephedrus) niger Gautier, Bonnamour \& Gaumont, 1929

Nomenclature:

?aphidivora (Rondani, 1848, Alysia); synonymy by Papp (1996a)

campestris Starý, 1962

Distribution: England

Notes: added by Van Veen et al. (2008)

Ephedrus (Ephedrus) persicae Froggat, 1904

Nomenclature:

nevadensis Baker, 1909

nitidus Gahan, 1917

vidali Quilis, 1931

interstitialis Watanabe, 1941

pulchellus Stelfox, 1941

impressus Granger, 1949

holmani Starý, 1958

palaestinensis Mackauer, 1959

Distribution: England, Ireland

Ephedrus (Ephedrus) plagiator (Nees, 1811)

Nomenclature:

Bracon plagiator Nees, 1811

parcicornis (Nees, 1834, Aphidius)

japonicus Ashmead, 1906

homostigma Fahringer, 1934 
Distribution: England, Scotland, Wales, Ireland

Subgenus Lysephedrus Starý, 1958

Ephedrus (Lysephedrus) validus (Haliday, 1833)

Nomenclature:

Aphidius validus Haliday, 1833

Distribution: England, Ireland

Genus Toxares Haliday, 1840

Nomenclature:

TRIONYX Haliday, 1833 preocc.

TERONYX Haldeman, 1842

Toxares deltiger (Haliday, 1833)

Nomenclature:

Aphidius deltiger Haliday, 1833

flaveolus (Györfi, 1958, Ephedrus)

Distribution: England, Ireland

Tribe Praini Mackauer, 1961

Genus Areopraon Mackauer, 1959

Nomenclature:

MESOPRAON Starý, 1981; synonymy by Tomanovic et al. (2006)

Areopraon lepelleyi (Waterston, 1926)

Nomenclature:

Praon lepelleyi Waterston, 1926

Distribution: England

Areopraon silvestre (Starý, 1971)

Nomenclature:

Praon silvestre Starý, 1971 
Distribution: Wales

Notes: Added by Baker and Broad (2013); transferred to Areopraon by Tomanovic et al. (2006).

\section{Genus Dyscritulus Hincks, 1943}

\section{Nomenclature:}

DYSCRITUS Marshall, 1896 preocc.

Notes: Dyscritus suffolciensis Morley, 1933 is listed by default as a species of Dyscritulus in Taxapad (Yu et al. 2012) as, although Starý (1959) recognised it as belonging to Euphorinae, its identity had not yet been established. Here it is synonymised under Syntretus splendidus (Marshall) q.v.

\section{Dyscritulus planiceps (Marshall, 1896)}

Nomenclature:

Dyscritus planiceps Marshall, 1896

Distribution: England, Wales

Dyscritulus pygmaeus Mackauer, 1961

\section{Genus Praon Haliday, 1833}

Nomenclature:

ACHORISTUS Ratzeburg, 1852

APHIDARIA Provancher, 1886

PARAPRAON Starý, 1983

Notes: species of Praon excluded from the British and Irish list:

[simulans (Provancher, 1886, Aphidaria); syn. aguti Smith, 1944] A Nearctic species, listed by Kloet and Hincks (1945) in error.

\section{Praon abjectum (Haliday, 1833)}

Nomenclature:

Aphidius abjectum Haliday, 1833

aphidiiforme (Ratzeburg, 1852, Bracon)

peregrinum Ruthe, 1859

Distribution: England, Ireland 
Praon absinthii Bignell, 1894

Notes: Erroneously listed in Taxapad as a junior synonym of flavinode; see, for example, Kavallieratos et al. (2005).

\section{Praon barbatum Mackauer, 1967}

Distribution: England

Notes: BMNH, det. Torrance \& Broad, added here

Praon bicolor Mackauer, 1959

Distribution: Wales

Notes: added by Baker and Broad (2009)

Praon cavariellae Starý, 1971

Distribution: Scotland, Wales

Notes: added by Baker and Broad (2013)

Praon dorsale (Haliday, 1833)

Nomenclature:

Aphidius dorsale Haliday, 1833

discolor (Nees, 1834, Blacus)

collare Förster, 1867 nom. nud.

Distribution: England, Wales, Ireland

Praon exsoletum (Nees, 1811)

Nomenclature:

Bracon exsoletum Nees, 1811

palitans Muesebeck, 1956

\section{Praon flavinode (Haliday, 1833)}

Nomenclature:

Aphidius flavinode Haliday, 1833

emacerator (Nees, 1834, Blacus)

glabrum Starý \& Schlinger, 1967 
Distribution: England, Wales

\section{Praon gallicum Starý, 1971}

Distribution: England

Notes: added by Traugott et al. (2008)

\section{Praon longicorne (Marshall, 1896)}

Nomenclature:

Aphidius longicorne Marshall, 1896

grossum Starý, 1971

Distribution: Wales

\section{Praon necans Mackauer, 1959}

Nomenclature:

nympheae Subba Rao, Sarup \& Sharma, 1963

Distribution: Wales

Notes: added by Baker and Broad (2013)

\section{Praon spinosum Mackauer, 1959}

Distribution: Wales

Notes: added by Baker and Broad (2009)

\section{Praon volucre (Haliday, 1833)}

Nomenclature:

Aphidius volucre Haliday, 1833

angulator (Nees, 1834, Blacus)

aphidivorum (Ratzeburg, 1844, Aphidius)

pruni Ivanov, 1925

breve Fahringer, 1935

mongolicum Watanabe, 1949

myzophagum Mackauer, 1959

Distribution: England, Wales, Ireland 
Praon yomenae Takada, 1968

Distribution: Wales

Notes: added by Baker and Broad (2013)

\section{Brachistinae}

\section{Subfamily Brachistinae Förster, 1863}

\section{Nomenclature:}

CALYPTINAE Marshall, 1872

Notes: Following Belshaw and Quicke (2002), Achterberg (2003a) and Belokobylskij et al. (2003) we treat the Brachistinae as a subfamily separate from Helconinae. Sharanowski et al. (2011) expanded the limits of the Brachistinae by including the tribe Diospilini and the former subfamily Blacinae (as well as the extralimital Brulleiini). The tribes employed here are those that have been used in previous classifications under the former subfamilies, but the tribal classification remains essentially untested.

\section{Tribe Blacini Förster, 1863}

Notes: Generally considered to be a distinct subfamily but Sharanowski et al. (2011) found that the blacines nested within the Brachistinae in their molecular phylogenetic analyses. We think it likely that these results will be upheld with further phylogenetic work and follow Sharanowski et al.'s recommended changes to the subfamily classification. Distribution and taxonomic data from Achterberg (1988a), with additional references given. Note that Tobias (1986) records B. (Neoblacus) koenigi Fischer, 1967 from England but there is no evidence that this species occurs here.

\section{Genus Blacometeorus Tobias, 1976}

\section{Blacometeorus brevicauda (Hellén, 1958)}

Nomenclature:

Diospilus brevicauda Hellén, 1958

Distribution: England

Notes: added by Achterberg (1988a); Shaw (1996b) 
Blacometeorus pusillus (Hellén, 1958)

Nomenclature:

Diospilus pusillus Hellén, 1958

Distribution: England

Notes: added by Shaw (1996b)

Genus Blacus Nees, 1818

Subgenus Blacus Nees, 1818

Nomenclature:

GONIOCORMUS Förster, 1863

MIOCOLUS Förster, 1863

Blacus (Blacus) errans (Nees, 1811)

Nomenclature:

Bracon errans Nees, 1811

vagans Ruthe, 1861

Distribution: England

Blacus (Blacus) exilis (Nees, 1811)

Nomenclature:

Bracon exilis Nees, 1811

lactucaphis (Fitch, 1855, Aphidius)

pallipes (Förster, 1863, Miocolus)

pallidipes (Dalla Torre, 1898, Miocolus)

nanus Ashmead, 1905 nom. nud.

propallipes Shenefelt, 1969

intermedius Janzon, 1975

Distribution: England, Ireland

Blacus (Blacus) filicornis Haeselbarth, 1973

Distribution: England, Ireland 
Blacus (Blacus) forticornis Haeselbarth, 1973

Distribution: England

Blacus (Blacus) hastatus Haliday, 1835

Nomenclature:

terebrator Ruthe, 1861

Distribution: England, Scotland, Ireland

Blacus (Blacus) humilis (Nees, 1811)

Nomenclature:

Bracon humilis Nees, 1811

trivialis Haliday, 1835

wesmaeli Ruthe, 1861

Distribution: England, Wales, Ireland

Blacus (Blacus) instabilis Ruthe, 1861

Nomenclature:

petiolatus Haeselbarth, 1973 nom. nud.

Distribution: England, Scotland, Ireland

Blacus (Blacus) leptostigma Ruthe, 1861

Distribution: Ireland

Blacus (Blacus) longipennis (Gravenhorst, 1809)

Nomenclature:

Ophion longipenne Gravenhorst, 1809

dubius Ruthe, 1861

Distribution: England, Scotland, Ireland

Blacus (Blacus) nigricornis Haeselbarth, 1973

Distribution: England, Scotland, Ireland 
Blacus (Blacus) paganus Haliday, 1835

Nomenclature:

brevicornis Ruthe, 1861

Distribution: Ireland

Blacus (Blacus) stelfoxi Haeselbarth, 1973

Distribution: England

Notes: The holotype was collected in Sandhurst, Kent (Haeselbarth 1973) but not listed for Britain or Ireland by Achterberg (1988a).

Subgenus Ganychorus Haliday, 1835

Blacus (Ganychorus) ambulans Haliday, 1835

Distribution: Ireland

Blacus (Ganychorus) armatulus Ruthe, 1861

Distribution: England

Blacus (Ganychorus) diversicornis Nees, 1834

Nomenclature:

compar Ruthe, 1861

Distribution: England, Ireland

Blacus (Ganychorus) macropterus Haeselbarth, 1973

Distribution: England, Scotland

Notes: Described as a subspecies of ambulans by Haeselbarth (1973), but not included by Huddleston (1978), where it should have been listed as a synonym. Treated as a separate species by Achterberg (1997).

Blacus (Ganychorus) maculipes Wesmael, 1835

Distribution: England, Scotland, Ireland 
Blacus (Ganychorus) nitidus Haeselbarth, 1973

Nomenclature:

petiolatus Tobias, 1976

Distribution: England

\section{Blacus (Ganychorus) pallipes Haliday, 1835}

Nomenclature:

barynoti misident.

tuberculatus Wesmael, 1835

florus Goureau, 1851

Distribution: England, Ireland

Notes: Included, as barynoti (Boudier, 1834) (a junior synonym of Pygostolus sticticus), as a doubtfully placed species of Blacus by Huddleston (1978).

\section{Blacus (Ganychorus) ruficornis (Nees, 1811)}

Nomenclature:

Bracon ruficornis Nees, 1811

bisstigmatus (Say, 1836, Microgaster)

tipulator (Zetterstedt, 1838, Bracon)

cerealis (Curtis, 1860, Dacnusa)

pallidipes (Costa, 1885, Dinocampus)

dentatus Hellén, 1958

Distribution: England, Scotland, Wales, Ireland

\section{Blacus (Ganychorus) strictus Stelfox, 1941}

Nomenclature:

strictus (Curtis, 1837, Ganychorus) nom. nud.

Distribution: Scotland, Ireland

Blacus (Ganychorus) tripudians Haliday, 1835

Distribution: England, Ireland 
Subgenus Hysterobolus Viereck, 1913

Blacus (Hysterobolus) mamillanus Ruthe, 1861

Nomenclature:

aptenodytes Marshall, 1889

Distribution: England

Tribe Brachistini Förster, 1863

Genus Eubazus Nees, 1814

Subgenus Aliolus Say, 1836

Eubazus (Aliolus) lepidus (Haliday, 1835)

Nomenclature:

Helcon lepidus Haliday, 1835

hofferi (Šnoflák, 1953, Triaspis); synonymy by Achterberg (2003a)

kusarensis (Abdinbekova, 1969, Allodorus); synonymy by Achterberg (2003a)

Distribution: England

Subgenus Allodorus Förster, 1863

Eubazus (Allodorus) convexope van Achterberg, 2000

Distribution: Scotland

Notes: added by Achterberg (2000)

Eubazus (Allodorus) semirugosus (Nees, 1816)

Nomenclature:

Sigalphus semirugosus Nees, 1816

rufipes (Herrich-Schäffer, 1838, Eubadizon)

tuberculator (Zetterstedt, 1838, Bracon)

curculionum (Hartig, 1847, Sigalphus)

atricornis (Ratzeburg, 1848, Brachistes)

mucronatus (Thomson, 1892, Calyptus) 


$$
\begin{aligned}
& \text { truncatus (Thomson, 1892, Calyptus) } \\
& \text { glabratus (Fahringer, 1941, Calyptus) } \\
& \text { arete (Fahringer, 1944, Calyptus) }
\end{aligned}
$$

Distribution: England, Scotland

Eubazus (Allodorus) tricoloripes van Achterberg, 2000

Distribution: England

Notes: added by Achterberg (2000)

Subgenus Brachistes Wesmael, 1835

Eubazus (Brachistes) fasciatus (Nees, 1816)

Nomenclature:

Sigalphus fasciatus Nees, 1816

fuscipalpis (Wesmael, 1835, Brachistes)

Distribution: England, Ireland

Eubazus (Brachistes) minutus (Ratzeburg, 1848)

Nomenclature:

Brachistes minutus Ratzeburg, 1848

Distribution: England

Eubazus (Brachistes) ruficoxis (Wesmael, 1835)

Nomenclature:

Brachistes ruficoxis Wesmael, 1835

politus (Ratzeburg, 1852, Brachistes); synonymy by van Achterberg in Belokobylskij et al. (2003)

byctisci (Watanabe, 1933, Calyptus)

ruficornis misspelling

Distribution: England

Eubazus (Brachistes) segmentatus (Marshall, 1889)

Nomenclature:

Calyptus segmentatus Marshall, 1889 
Distribution: England

Eubazus (Brachistes) semicastaneus (Marshall, 1893)

Nomenclature:

Calyptus semicastaneus Marshall, 1893

Distribution: England

Eubazus (Brachistes) tibialis (Haliday, 1835)

Nomenclature:

Helcon tibialis Haliday, 1835

uncigenus (Wesmael, 1835, Brachistes)

Distribution: England, Scotland, Ireland

Subgenus Calyptus Haliday, 1835

Eubazus (Calyptus) macrocephalus Nees, 1812

Nomenclature:

synchitae (Hedqvist, 1956, Eubadizon)

ratzeburgi (Fischer, 1962, Eubadizon); synonymy by van Achterberg in Belokobylskij et al. (2003)

xiphydriae Tobias, 1986; synonymy by van Achterberg in Belokobylskij et al. (2003)

Distribution: England

Eubazus (Calyptus) sigalphoides (Marshall, 1889)

Nomenclature:

Calyptus sigalphoides Marshall, 1889

Distribution: England

Subgenus Eubazus Nees, 1814

Nomenclature:

Eubadizon Nees, 1834

Eubadizus Nees, 1834 
Eubazus (Eubazus) flavipes (Haliday, 1835)

Nomenclature:

Helcon flavipes Haliday, 1835

laevis (Herrich-Schäffer, 1838, Eubadizon)

Distribution: England, Ireland

Eubazus (Eubazus) longicauda (Curtis, 1832)

Nomenclature:

Zele longicauda Curtis, 1832

Distribution: England

Notes: Listed as a species of Zele (=Homolobus) in Huddleston (1978). Will be synonymised under another species of Eubazus (van Achterberg, pers. comm.).

\section{Eubazus (Eubazus) pallipes Nees, 1814}

Nomenclature:

coxalis (Nees, 1834, Eubadizon)

semistriatus (Haliday, 1835, Helcon) unavailable

americanus (Cresson, 1872, Eubadizon)

pallipede misspelling

pallidipes misspelling

pollipes misspelling

Distribution: England

\section{Genus Foersteria Szépligeti, 1896}

\section{Foersteria (Foersteria) laeviuscula Szépligeti, 1896}

Distribution: England, Scotland

Notes: NMS, det. van Achterberg, added here

Foersteria (Foersteria) puber (Haliday, 1835)

Nomenclature:

Helcon puber Haliday, 1835

opaca (Reinhard, 1867, Calyptus) 
flavipes Szépligeti, 1896

talitzkii Tobias, 1961

Distribution: England, Ireland

\section{Genus Schizoprymnus Förster, 1863}

Notes: species of Schizoprymnus excluded from the British and Irish list:

[nigripes (Thomson, 1892, Sigalphus)] Seems to have been recorded in error by Kloet and Hincks (1945), perpetuated by Huddleston (1978).

\section{Schizoprymnus ambiguus (Nees, 1816)}

Nomenclature:

Sigalphus ambiguus Nees, 1816

Distribution: England, Ireland

Schizoprymnus collaris (Thomson, 1874)

Nomenclature:

Sigalphus collaris Thomson, 1874

Distribution: England

Notes: Added by Notton et al. (2014); listed as a species of Triaspis by Yu et al. (2012) but transferred back to Schizoprymnus by Notton et al. (2014).

\section{Schizoprymnus obscurus (Nees, 1816)}

Nomenclature:

Sigalphus obscurus Nees, 1816

Distribution: England

\section{Genus Triaspis Haliday, 1838}

Nomenclature:

MUIRIELLA Fullaway, 1919

Notes: species of Triaspis excluded from the British and Irish list:

[striola (Thomson, 1874, Sigalphus)] Listed as a doubtfully British species in Huddleston (1978); the only record is of a tentative identification by Nixon (Hussey 1952). 


\section{Triaspis aciculata (Ratzeburg, 1848)}

Nomenclature:

Sigalphus aciculatus Ratzeburg, 1848

Distribution: England

Notes: BMNH, NMS, det. van Achterberg, added here; although synonymised with obscurella by van Achterberg (in Belokobylskij et al. 2003) this is listed as a separate species in Fauna Europaea. There are English specimens identified by van Achterberg as aciculata in NMS and BMNH.

Triaspis caledonica (Marshall, 1888)

Nomenclature:

Sigalphus caledonicus Marshall, 1888

Distribution: Scotland

Triaspis caudata (Nees, 1816)

Nomenclature:

Sigalphus caudatus Nees, 1816

gracilis (Herrich-Schäffer, 1840, Sigalphus)

australis (Szépligeti, 1901, Sigalphus)

arctica Hellén, 1958

Distribution: England, Ireland

\section{Triaspis flavipalpis (Wesmael, 1835)}

Nomenclature:

Sigalphus flavipalpis Wesmael, 1835

Distribution: England

Triaspis floricola (Wesmael, 1835)

Nomenclature:

Sigalphus floricola Wesmael, 1835

minima Snoflák, 1953

Distribution: England, Ireland 
Triaspis luteipes (Thomson, 1874)

Nomenclature:

Sigalphus luteipes Thomson, 1874

Distribution: England

Triaspis obscurella (Nees, 1816)

Nomenclature:

Sigalphus obscurellus Nees, 1816

simulator (Szépligeti, 1901, Sigalphus)

Distribution: England

Triaspis pallipes (Nees, 1816)

Nomenclature:

Sigalphus pallipes Nees, 1816

fulvipes (Haliday, 1835, Helcon)

fagi (Ratzeburg, 1852, Brachistes)

similis (Szépligeti, 1901, Sigalphus) preocc.

pallidipes misspelling

Distribution: England, Ireland, Isle of Man

Notes: some distribution data from Shaw and Askew (1976)

Triaspis podlussanyi Papp, 1998

Distribution: England

Notes: added by Shaw \& Mendel (in prep.)

Triaspis striatula (Nees, 1816)

Nomenclature:

Sigalphus striatulus Nees, 1816

Distribution: England, Ireland

Triaspis thoracica (Curtis, 1860)

Nomenclature:

Sigalphus thoracicus Curtis, 1860 
gibberosa (Szépligeti, 1901, Sigalphus); synonymy by Papp (2004b)

rugosa (Szépligeti, 1901, Sigalphus); synonymy by Papp (2004b)

Distribution: England

Tribe Diospilini Förster, 1863

Genus Aspicolpus Wesmael, 1838

Nomenclature:

ASPIDOCOLPUS Agassiz, 1846

\section{Aspicolpus ? clipealis (Tobias, 1967)}

Nomenclature:

Aspidocolpus clipealis Tobias, 1967

Distribution: England

Notes: NMS, det. van Achterberg, added here; tentative identification; included here as a certain generic record of an Aspicolpus species in Britain.

\section{Genus Aspigonus Wesmael, 1835}

Nomenclature:

ASPIDOGONUS Agassiz, 1846

\section{Aspigonus flavicornis (Nees, 1834)}

Nomenclature:

Bracon flavicornis Nees, 1834

diversicornis Wesmael, 1835; synonymy by van Achterberg in Belokobylskij et al. (2003)

Distribution: England, Wales

\section{Genus Diospilus Haliday, 1833}

Nomenclature:

BAEACIS Förster, 1878; synonymy by Achterberg (2014)

ALLOCHROMUS Marshall, 190 
Diospilus abietis (Ratzeburg, 1844)

Nomenclature:

Aspigonus abietis Ratzeburg, 1844

Diospilus capito (Nees, 1834)

Nomenclature:

Bracon capito Nees, 1834

filator (Nees, 1834, Bracon) preocc.

fuscipes (Wesmael, 1835, Taphaeus)

Distribution: England, Ireland

Diospilus dispar (Nees, 1811)

Nomenclature:

Bracon dispar Nees, 1811

ephippium (Nees, 1834, Bracon)

Distribution: England

Diospilus inflexus Reinhard, 1862

Nomenclature:

ovatus Marshall, 1889

Distribution: England

Diospilus intermedius (Förster, 1878)

Nomenclature:

Baeacis intermedia Förster, 1878

Distribution: England

Notes: BMNH, det. van Achterberg, added here

Diospilus morosus Reinhard, 1862

Distribution: England 
Diospilus nigricornis (Wesmael, 1835)

Nomenclature:

Taphaeus nigricornis Wesmael, 1835

affinis (Wesmael, 1835, Taphaeus)

rufipes Reinhard, 1862

Distribution: England

Notes: Listed as Taphaeus affinis and T. nigricornis in Huddleston (1978).

Diospilus oleraceus Haliday, 1833

Nomenclature:

conformis (Wesmael, 1835, Taphaeus)

ruficornis Szépligeti, 1896

Distribution: England, Ireland

Diospilus productus Marshall, 1894

Distribution: England

\section{Genus Taphaeus Wesmael, 1835}

Nomenclature:

Anostenus Förster, 1863

Anastenus Dalla Torre, 1898

Notes: The systematic position of Taphaeus is uncertain. Treated as a genus of Blacinae in Fauna Europaea.

Taphaeus hiator (Thunberg, 1824)

Nomenclature:

Ichneumon hiator Thunberg, 1824

irregularis Wesmael, 1835

speculator (Haliday, 1835, Helcon)

polydrusi (Gahan, 1916, Diospilus)

Distribution: England, Scotland, Ireland 
Genus Vadumasonium Kammerer, 2006

Nomenclature:

VADUM Mason, 1987 preocc.

Vadumasonium vardyorum van Achterberg \& Broad, 2013

Distribution: England

Notes: added by Achterberg and Broad (2013)

Tribe Dyscoletini van Achterberg, 1984

Notes: This is generally considered to be a tribe of Blacinae; we list the Dyscoletini as a tribe of Brachistinae because we follow Sharanowski et al. (2011) in including the Blacinae within Brachistinae.

\section{Genus Dyscoletes Haliday, 1840}

Nomenclature:

DYSCOLUS Haliday, 1836 preocc.

DISCOLUS misspelling

MICROCENTRUS Szépligeti, 1904 preocc.

ELACHISTOCENTRUM Schulz, 1911

Dyscoletes lancifer (Haliday, 1836)

Nomenclature:

Dyscolus lancifer Haliday, 1836

similis (Szépligeti, 1896, Discoletes)

Distribution: England, Scotland

\section{Braconinae}

Subfamily Braconinae Nees, 1812

Notes: Tribal classification follows Belshaw et al. (2001) and Quicke (pers. comm.). For genera other than Bracon and Coeloides, Shaw (1994) and Shaw and Quicke (1999) give distribution data. 
Tribe Aphrastobraconini Ashmead, 1900

\section{Genus Atanycolus Förster, 1862}

Notes: species excluded from the British and Irish list by Shaw and Quicke (1999):

[denigrator (Linnaeus, 1758, Ichneumon); syn. incertus (Sulzer, 1776, Ichneumon); heteropus (Thomson, 1892, Bracon) albiscutis Telenga, 1936]

\section{Genus Cyanopterus Haliday, 1835}

Notes: species excluded from the British and Irish list by Shaw and Quicke (1999):

[flavator (Fabricius, 1793, Ichneumon); syn. flavulator (Ratzeburg, 1844, Bracon); longipalpis (Thomson, 1892, Bracon); barcinonensis (Marshall, 1897, Coeloides)]

Genus Pseudovipio Szépligeti, 1896

Pseudovipio guttiventris (Thomson, 1892)

Nomenclature:

Bracon guttiventris Thomson, 1892

variegatus (Boheman, 1853, Agathis) preocc.

biroi Szépligeti, 1896

Distribution: England

Notes: Listed as Glyptomorpha variegata in Huddleston (1978).

\section{Genus Vipio Latreille, 1804}

Notes: species excluded from the British and Irish list by Shaw and Quicke (1999):

[terrefactor (Villers, 1789, Ichneumon); syn. improvisus Kokujev, 1898; interpellator Kokujev, 1898; neesii Kokujev, 1898] The only record was due to a misidentification of Pseudovipio guttiventris (Shaw and Quicke 1999).

Tribe Braconini Nees, 1812

Genus Baryproctus Ashmead, 1900

Nomenclature:

Barycryptus Hoffmeyer, 1932 


\section{Baryproctus barypus (Marshall, 1885)}

Nomenclature:

Bracon barypus Marshall, 1885

hungaricus Szépligeti, 1901

caucasicus Telenga, 1936

apti Györfi, 1953

Distribution: England

Notes: See Shaw (1994) and Jennings (2008) for recent records.

\section{Genus Bracon Fabricius, 1804}

Notes: Comprehensive taxonomic revisions of Bracon are lacking for all but a few species groups. Published works differ substantially in their treatments of valid species, synonyms and subgeneric placements (compare, for example, the differing treatments of Tobias (1986), Papp (1974), Papp (1996a), and Shenefelt (1978)) so the taxonomy in Taxapad (Yu et al. 2012) has, for the most part, been used as a default classification.

\section{Subgenus Bracon Fabricius, 1804}

\section{Nomenclature:}

BRACO Wesmael, 1838

BRACHON Agassiz, 1846

TROPIDOBRACON Ashmead, 1900

MICROBRACON Ashmead, 1890

AMICOPLIDEA Ashmead, 1900

MACRODYCTIUM Ashmead, 1900

LIOBRACON Nason, 1905 preocc.

LORENZOA de Stefani-Perez, 1909

SELIODUS Brèthes, 1909

KULCZYNSKIA Niezabitowski, 1910

BRAZON Schulz, 1911

STRIOBRACON Fahringer, 1927

CHIVINIA Shestakov, 1932

EUTROPOBRACON Ayyar, 1928 
Bracon (Bracon) alutaceus Szépligeti, 1901

Nomenclature:

pygmaeus Niezabitowski, 1910; synonymy by Papp (2008a)

polonicus Fahringer, 1927; synonymy by Papp (2008a)

pallidalatus Tobias, 1957

Distribution: England, Scotland

Notes: NMS, det. Papp, added here

Bracon (Bracon) flavipes Nees, 1834

Distribution: England

\section{Bracon (Bracon) fulvipes Nees, 1834}

Nomenclature:

carinatus Szépligeti, 1901

marshalli Vayssière, 1902 preocc.

glabratus Fahringer, 1927

apionis Strand, 1928

sylvanus Greese, 1928

kiritshenkoi Telenga, 1936

Distribution: England, Scotland, Wales, Ireland, Isle of Man

\section{Bracon (Bracon) intercessor Nees, 1834}

\section{Nomenclature:}

laetus (Wesmael, 1838, Braco); synonymy by Papp (2012), as a 'var' of intercessor

lativentris Thomson, 1892 preocc.

fulvus Szépligeti, 1896

universitatis Dalla Torre, 1898

adjectus Szépligeti, 1901

bisinuatus Szépligeti, 1901

dubiosus Szépligeti, 1901

duplicatus Szépligeti, 1901

fallaciosus Szépligeti, 1901 
elegans Szépligeti, 1901

mixtus Szépligeti, 1901

mundus Szépligeti, 1901

nigropictus Szépligeti, 1901

nitidiusculus Szépligeti, 1901

rufiscapus Szépligeti, 1901

subtilis Szépligeti, 1901

suspectus Szépligeti, 1901

vigilax Kokujev, 1912

maidli Fahringer, 1925

asiaticus Fahringer, 1927 preocc.

concolor Fahringer, 1927 preocc.

major Fahringer, 1927

concorellus Strand, 1928

megasomides Strand, 1928

rhynchiti Greese, 1928

kachetinus Telenga, 1933

kansensis Fahringer, 1934 preocc.

maslovskii Telenga, 1936

segregatus Telenga, 1936

Distribution: England, Scotland

\section{Bracon (Bracon) leptus Marshall, 1897}

\section{Nomenclature:}

centaureae Szépligeti, 1901

rufipedator Szépligeti, 1901

rufipalpis Szépligeti, 1901]

Distribution: England

Notes: Listed as British in Fauna Europaea, probably because it was described by Marshall, but the first published British record was by Jennings (2012). 
Bracon (Bracon) longicollis (Wesmael, 1838)

Nomenclature:

Braco longicollis Wesmael, 1838

fraudator Marshall, 1885

brevicauda Thomson, 1892 preocc.

crassicauda Thomson, 1892

pseudowesmaeli Strand, 1928

wesmaeli Fahringer, 1927 preocc.

Distribution: England, Scotland, Wales, Isle of Man

Notes: spme distribution data from Papp (1999c)

Bracon (Bracon) luteator Spinola, 1808

Nomenclature:

nigripedator Nees, 1834

filicauda Costa, 1888

hypopygialis Szépligeti, 1901

intermedius Szépligeti, 1901

pilosulus Szépligeti, 1901

Distribution: England

Notes: added by Fulmek (1968) (omitted by Huddleston 1978)

Bracon (Bracon) nigratus (Wesmael, 1838)

Nomenclature:

Braco nigratus Wesmael, 1838

Distribution: England, Ireland

Bracon (Bracon) pectoralis (Wesmael, 1838)

Nomenclature:

Braco pectoralis Wesmael, 1838

ochrosus Szépligeti, 1896

sulphurator Szépligeti, 1896

unicolor Szépligeti, 1896 
fumigatus Szépligeti, 1901; synonymy by Papp (2008a)

Distribution: England

\section{Bracon (Bracon) rugulosus Szépligeti, 1901}

Nomenclature:

depressiusculus Szépligeti, 1901

neglectus Szépligeti, 1904

spurnensis Hincks, 1951

Distribution: England, Scotland, Wales

Notes: According to Taxapad (Yu et al. 2012), rugulosus Szépligeti is preoccupied by Bracon rugulosus Nees, 1811 (now classified as a species of Aleiodes, in Rogadinae).

Omitted by Huddleston (1978).

Bracon (Bracon) scutellaris (Wesmael, 1838)

Nomenclature:

Braco scutellaris Wesmael, 1838

Distribution: England, Scotland, Ireland

Bracon (Bracon) speerschneideri Schmiedeknecht, 1897

Distribution: England

Notes: added by Papp (1999a)

Bracon (Bracon) subrugosus Szépligeti, 1901

Nomenclature:

sulcatulus Szépligeti, 1896

subglaber Szépligeti, 1901

quinquemaculatus Szépligeti, 1901

trypetanus Fahringer, 1927; synonymy by Papp (2008a)

tauricus Telenga, 1936

Distribution: England

Notes: added by Papp (2008a) 


\section{Bracon (Bracon) trucidator Marshall, 1888}

\section{Nomenclature:}

minutator misident.

bilineatus Thomson, 1892

hilaris Marshall, 1897

pannonicus Szépligeti, 1901

marshalli Telenga, 1936 preocc.

Distribution: England

Notes: BMNH, NMS, added here; previously confused under minutator (van Achterberg, pers. comm.). Both species are present in the BMNH and NMS collections, det. Papp and van Achterberg.

\section{Bracon (Bracon) variegator Spinola, 1808}

\section{Nomenclature:}

melanosoma Szépligeti, 1901

micros Szépligeti, 1901

nanulus Szépligeti, 1901

lineatellae (Fischer, 1968, Habrobracon); synonymy by Papp (2008b)

Distribution: England, Scotland, Ireland

\section{Subgenus Glabrobracon Fahringer, 1927}

Notes: Some taxonomic and distribution data from Papp (1999a), Papp (1999c), Papp (2000).

Species of Bracon (Glabrobracon) excluded from the British and Irish list:

[coniferarum Fahringer, 1928] Should not have been listed by Huddleston (1978) as it was recorded as British only on the basis of Carr's Staffordshire lists (see Perkins 1953, Fitton et al. 1978, Shaw 2003a).

\section{Bracon (Glabrobracon) abbreviator Nees, 1834}

\section{Nomenclature:}

abscissor Nees, 1834; synonymy by Papp (2008a)

oestmaeli (Wesmael, 1838, Braco); synonymy by Papp (2008a)

regularis (Wesmael, 1838, Braco); synonymy by Papp (2008a) 
eutrephes Marshall, 1897

rufigaster Szépligeti, 1901; synonymy by Papp (2008a)

rufiventris Telenga, 1936 preocc.

minimula Strand, 1928

minimus Fahringer, 1927 preocc.

Distribution: England

Bracon (Glabrobracon) admotus Papp, 2000

Distribution: England

Notes: BMNH, det. Papp, added here

Bracon (Glabrobracon) albion Papp, 1999

Distribution: England, Scotland

Notes: added by Papp (1999c)

Bracon (Glabrobracon) arcuatus Thomson, 1892

Distribution: England, Scotland

Notes: Added by Papp (2000); listed by Kloet and Hincks (1945) (but not by Huddleston 1978), although it is not clear on what basis.

Bracon (Glabrobracon) atrator Nees, 1834

Nomenclature:

longicauda Thomson, 1892

Distribution: England, Scotland, Ireland

Bracon (Glabrobracon) caudatus Ratzeburg, 1848

Distribution: England

Bracon (Glabrobracon) claripennis Thomson, 1892

Distribution: England

Notes: added by Papp (2000) 
Bracon (Glabrobracon) colpophorus (Wesmael, 1838)

Nomenclature:

Braco colpophorus Wesmael, 1838

mokrzeckii Niezabitowski, 1927; synonymy by Papp (1997)

Distribution: England

Bracon (Glabrobracon) conjugellae Bengtsson, 1924

Nomenclature:

bengtssoni Fahringer, 1928 preocc.

minor Fahringer, 1928 preocc.

nanana Strand, 1928

Distribution: England, Scotland

Notes: NMS, det. Papp, added here

Bracon (Glabrobracon) curticaudis Szépligeti, 1901

Distribution: Scotland

Notes: Added by Papp (2008a) and removed from synonymy with terebella.

Bracon (Glabrobracon) delibator Haliday, 1833

Nomenclature:

anthracinus Nees, 1834; synonymy by Achterberg (1997)

breviseta Fahringer, 1935

Distribution: England, Scotland, Ireland

Bracon (Glabrobracon) fuscicoxis (Wesmael, 1838)

Nomenclature:

Braco fuscicoxis Wesmael, 1838

levicarinatus Niezabitowski, 1910

Distribution: England 


\section{Bracon (Glabrobracon) glaphyrus Marshall, 1897}

Distribution: England

Notes: Faure (1924) mentions English material but glaphyrus was omitted by Huddleston (1978).

\section{Bracon (Glabrobracon) guttator Panzer, 1804}

Distribution: England

Notes: Omitted by Huddleston (1978); recorded as British by Fulmek (1968), although we are not sure how reliably; English material in NMS.

Bracon (Glabrobracon) immutator Nees, 1834

Nomenclature:

efoveolatus Thomson, 1892

hemirugosus Szépligeti, 1901

flavicoxanus Strand, 1928

marshalli Fahringer, 1927 preocc.

romani Fahringer, 1927 preocc.

uplandiae Strand, 1928

austriacus Fahringer, 1936

nigripalpis Telenga, 1936

Distribution: England, Scotland

Bracon (Glabrobracon) instabilis Marshall, 1897

Distribution: England

Bracon (Glabrobracon) kopelkei Papp, 2000

Distribution: England

Notes: BMNH, det. Papp, added here

Bracon (Glabrobracon) longulus Thomson, 1892

Distribution: England, Scotland

Notes: NMS, det. Papp, added here 
Bracon (Glabrobracon) marshalli Szépligeti, 1901

Nomenclature:

obscurator misident.

Distribution: England, Scotland

Notes: Added by Papp (2000); listed by Kloet and Hincks (1945) (but not by Huddleston 1978), although it is not clear on what basis.

Bracon (Glabrobracon) minutator (Fabricius, 1798)

Nomenclature:

Ichneumon minutator Fabricius, 1798

thalassinus Schmiedeknecht, 1897; synonymy by Papp (1999a)

tener Szépligeti, 1904

abscissoris Strand, 1928

flavipalpula Strand, 1928

flavipalpis Fahringer, 1928 preocc.

rufiventris Fahringer, 1928 preocc.

notatus Telenga, 1936

unicolor Telenga, 1936 preocc.

Distribution: England, Ireland

Notes: Braconminutator auct. is trucidator Marshall (van Achterberg, pers. comm.).

Bracon (Glabrobracon) momphae Papp, 1999

Distribution: England, Scotland

Notes: added by Papp (1999c)

Bracon (Glabrobracon) nigricollis (Wesmael, 1838)

Nomenclature:

Braco nigricollis Wesmael, 1838

brunneomaculatus Schütze \& Roman, 1931

Distribution: England

Notes: NMS, det. Papp, added here 
Bracon (Glabrobracon) obscurator Nees, 1811

Nomenclature:

kotulai Niezabitowski, 1910

nigripes Fahringer, 1928 preocc.

rytrensis Fahringer, 1928 preocc.

rytronis Strand, 1928

zaleszczykiensis Strand, 1928

Distribution: England, Scotland, Wales

Bracon (Glabrobracon) otiosus Marshall, 1885

Nomenclature:

macrurus Thomson, 1892; synonymy by Papp (1999c)

explorator Szépligeti, 1904 preocc.; synonymy by Papp (1999c)

pumilionis Roman, 1928; synonymy by Papp (1999c)

Distribution: England

Notes: Treated as a synonym of bipartitus (=variator) by Belokobylskij et al. (2003).

Bracon (Glabrobracon) pallicarpus Thomson, 1892

Distribution: England

Notes: added by Papp (2000)

Bracon (Glabrobracon) parvicornis Thomson, 1892

Nomenclature:

carbonarius Szépligeti, 1901 preocc.

aterrimus Telenga, 1936 preocc.

Distribution: England

Notes: NMS, det. Papp, added here

Bracon (Glabrobracon) parvulus Wesmael, 1838

Nomenclature:

fumipennis Thomson, 1892

fuscipennis Thomson, 1892 preocc. 
thomsoni Marshall, 1897

Distribution: England, Scotland, Wales

Notes: NMS, det. Papp, added here

Bracon (Glabrobracon) pineti Thomson, 1892

Distribution: England

Notes: NMS, det. Papp, added here

Bracon (Glabrobracon) praecox (Wesmael, 1838)

Nomenclature:

Braco praecox Wesmael, 1838

biorrhizae Fahringer, 1928

elongatus Dutu-Lacatusu, 1956 preocc.

Distribution: Scotland

Notes: NMS, det. Papp, added here

Bracon (Glabrobracon) ratzeburgii Dalla Torre, 1898

Nomenclature:

longicaudis Ratzeburg, 1852 preocc.

Distribution: England

Bracon (Glabrobracon) terebella (Wesmael, 1838)

Nomenclature:

Braco terebella Wesmael, 1838

breviterebris Fahringer, 1928

miaricola Strand, 1928

wesmaeli Fahringer, 1928 preocc.

unicolor Telenga, 1936 preocc.

Distribution: England 


\section{Bracon (Glabrobracon) variator Nees, 1811}

\section{Nomenclature:}

bipartitus (Wesmael, 1838, Braco)

maculiger (Wesmael, 1838, Braco)

collinus Szépligeti, 1901

breviventris Szépligeti, 1901

rytrensis Niezabitowski, 1910

sueciensis Fahringer, 1927

dimidiatus Fahringer, 1928 preocc.

nigerrimus Fahringer, 1928

rytrensis Fahringer, 1928 preocc.

rytrocola Strand, 1928

chinensis Fahringer, 1929 preocc.

caucasicus Telenga, 1936

collaris Telenga, 1936 preocc.

meridionalis Telenga, 1936

ornatulus Telenga, 1936

rytrensis Telenga, 1936 preocc.

turcmenus Telenga, 1936

asiaticus Telenga, 1949 preocc.

Distribution: England, Scotland, Wales, Ireland

Notes: Braco bipartitus is listed as a subspecies of variator in Taxapad (Yu et al. 2012).

Listed as a British species by Huddleston (1978) but this actually refers to otiosus.

\section{Bracon (Glabrobracon) xanthogaster Nees, 1834}

Nomenclature:

breviseta Hedwig, 1961 preocc.

Distribution: England

Notes: NMS, det. Papp, added here 
Subgenus Habrobracon Ashmead, 1895

Bracon (Habrobracon) concolorans Marshall, 1900

Nomenclature:

concolor Thomson, 1892 preocc.

nigricans (Szépligeti, 1901, Habrobracon); synonymy by Papp (2008b)

mongolicus (Telenga, 1936, Habrobracon)

Distribution: England, Scotland, Ireland

Notes: Added by Papp (2008b) and removed from synonymy with stabilis.

\section{Bracon (Habrobracon) crassicornis Thomson, 1894}

Nomenclature:

flavosignatus (Tobias, 1957, Habrobracon); synonymy by Papp (2008b)

Distribution: England, Scotland

Notes: added by Papp (2008b)

\section{Bracon (Habrobracon) hebetor Say, 1836}

\section{Nomenclature:}

dorsator Say, 1836

brevicornis (Wesmael, 1838, Braco)

juglandis Ashmead, 1889

brunneus (Szépligeti, 1901, Habrobracon)

vernalis (Szépligeti, 1901, Habrobracon)

beneficientior (Viereck, 1911, Habrobracon)

plotnicovi (Bogoljubov, 1914, Habrobracon)

breviantennatus de Stefani, 1919

serinopae (Cherian, 1929, Microbracon)

tortricidarum (Goidanich, 1934, Habrobracon)

pectinophorae (Watanabe, 1935, Habrobracon)

asiaticus (Telenga, 1936, Habrobracon)

flavus (Telenga, 1936, Habrobracon)

turkestanicus (Telenga, 1936, Habrobracon)

lozinskii (Bogacev, 1939, Habrobracon) 
Distribution: England

Notes: Mainly an indoors species in Britain, attacking pests of stored products; brevicornis been variously treated as a valid species or as a synonym of hebetor, being listed under the latter in Taxapad (Yu et al. 2012) and by Papp (2008b). Both brevicornis and hebetor have been recorded as British.

\section{Bracon (Habrobracon) stabilis (Wesmael, 1838)}

Nomenclature:

Braco stabilis Wesmael, 1838

opacus Stelfox, 1953 preocc.

Distribution: England, Ireland

\section{Subgenus Lucobracon Fahringer, 1927}

Notes: species of Bracon (Lucobracon) excluded from the British and Irish list:

[strobilorum Ratzeburg, 1848] England and Ireland are listed under the distribution in Shenefelt (1978), and Britain in Fauna Europaea, but we have been unable to trace any British or Irish literature records or specimens. In Belokobylskij et al. (2003) and in Taxapad (Yu et al. 2012) this is listed as a species of Coeloides but Papp (1992) had established that this species had been mis-interpreted and is in fact a species of Bracon (Lucobracon). Swiss specimens identified as strobilorum by Papp in NMS and BMNH belong in Bracon.

Bracon (Lucobracon) brachycerus Thomson, 1892

Nomenclature:

kudsiricus Papp, 1965

Distribution: England

Notes: NMS, det. Papp, added here

Bracon (Lucobracon) crassungula Thomson, 1892

Distribution: England

Notes: NMS, BMNH, det. Papp, added here

Bracon (Lucobracon) erraticus (Wesmael, 1838)

Nomenclature:

Braco erraticus Wesmael, 1838 


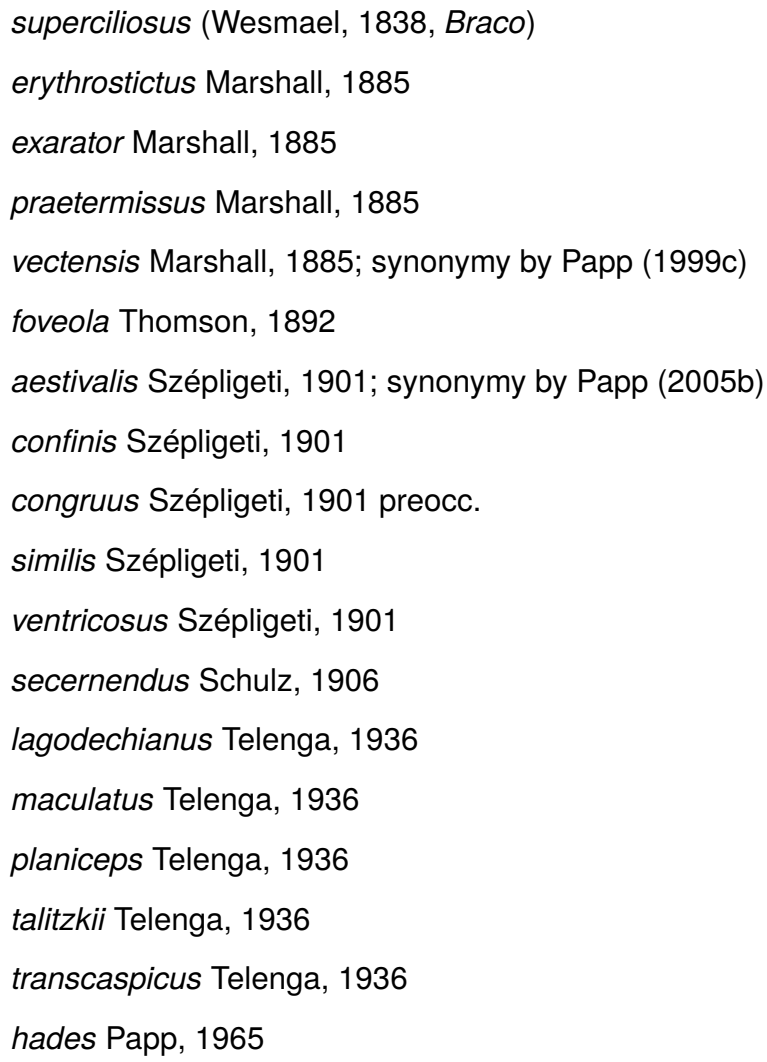

Distribution: England, Scotland, Ireland, Isle of Man

Notes: Although pratermissus was synonymised with Bracon erraticus by Papp (1999c), this has not been followed by Belokobylskij et al. (2003).

\section{Bracon (Lucobracon) flagellaris Thomson, 1892}

\section{Nomenclature:}

facialis Thomson, 1892 preocc.

thomsonii Dalla Torre, 1898 preocc.

dallatorrei Szépligeti, 1901

Distribution: England

Notes: BMNH, det. Papp, added here

\section{Bracon (Lucobracon) grandiceps Thomson, 1892}

\section{Nomenclature:}

gallicus Thomson, 1892 
Distribution: Scotland

Notes: NMS, BMNH, det. Papp, added here

Bracon (Lucobracon) guttiger (Wesmael, 1838)

Nomenclature:

Braco guttiger Wesmael, 1838

fasciatus Fahringer, 1927

Distribution: England, Scotland

Bracon (Lucobracon) hylobii Ratzeburg, 1848

Nomenclature:

bruchorum Fahringer, 1934

Distribution: England, Scotland

Bracon (Lucobracon) larvicida (Wesmael, 1838)

Nomenclature:

Braco larvicida Wesmael, 1838

crassiusculus Szépligeti, 1901

romani Fahringer, 1927 preocc.

szepligetii Fahringer, 1927 preocc.

fahringeriensis Strand, 1928

pseudoromani Strand, 1928

Distribution: England

Bracon (Lucobracon) nigriventris Wesmael, 1838

Nomenclature:

Braco nigriventris Wesmael, 1838

subornatus Szépligeti, 1901; synonymy by Papp (2008a)

biroi Fahringer, 1927

minor Fahringer, 1927

albanicus Telenga, 1936

laticeps Telenga, 1936

lencoranus Telenga, 1936 
persimilis Telenga, 1936

turolus Papp, 1984

Distribution: England

Notes: NMS, det. Papp, added here

Bracon (Lucobracon) sphaerocephalus Szépligeti, 1901

Nomenclature:

globiceps Szépligeti, 1901; synonymy by Papp (2005b)

Distribution: England, Scotland

Notes: NMS, det. Papp, added here

Bracon (Lucobracon) thuringiacus Schmiedeknecht, 1897

Nomenclature:

schmiedeknechti Fahringer, 1927 preocc.

blankenburgiae Strand, 1928

Distribution: England

Notes: added by Papp (1999a)

Bracon (Lucobracon) tornator Marshall, 1885

Nomenclature:

aequalis Thomson, 1892

Distribution: England, Scotland, Wales

Notes: some distribution data from Papp (1997)

Bracon (Lucobracon) triangularis Nees, 1834

Distribution: England, Ireland

\section{Subgenus Orthobracon Fahringer, 1927}

Notes: species of Bracon (Orthobracon) excluded from the British and Irish list:

[tenuicornis (Wesmael, 1838, Braco)] Probably not a valid British or Irish species now that $B$. (Lucobracon) erythrostictus has been taken out of synonymy (Papp 1999c). 
Bracon (Orthobracon) discoideus (Wesmael, 1838)

Nomenclature:

Braco discoideus Wesmael, 1838

opionus Fahringer, 1928

sculpturatus Fahringer, 1928

sculpturifera Strand, 1928

Distribution: England, Scotland, Ireland

Bracon (Orthobracon) epitriptus Marshall, 1885

Nomenclature:

pallidipes Szépligeti, 1896; synonymy by Papp (2008a)

melanogaster Szépligeti, 1901

Distribution: England, Scotland, Wales, Ireland

Notes: some distribution data from Papp (1999c)

\section{Bracon (Orthobracon) exhilarator Nees, 1834}

Nomenclature:

satanas (Wesmael, 1838, Braco)

tibialis Zetterstedt, 1838

striolatus Thomson, 1892

marshalli Fahringer, 1927 preocc,

polonicus Fahringer, 1927 preocc.

polonicella Strand, 1928

varicoloris Strand, 1928

Distribution: England, Scotland, Wales, Isle of Man

Bracon (Orthobracon) filicornis Thomson, 1892

Distribution: Scotland

Notes: NMS, det. Papp, added here

Bracon (Orthobracon) laevigatissimus Dalla Torre, 1898

Nomenclature: 
laevigatus Ratzeburg, 1852 preocc.

Distribution: England

Notes: Omitted by Huddleston (1978); listed as British by various authors dating back to Marshall (1885).

\section{Bracon (Orthobracon) mediator Nees, 1834}

Distribution: England, Scotland, Ireland

\section{Bracon (Orthobracon) ochropus Nees, 1834}

\section{Nomenclature:}

marshalli Fahringer, 1927 preocc.

neesi Fahringer, 1927

ochropodis Strand, 1928

Distribution: England, Scotland

Notes: NMS, det. Papp, added here

Bracon (Orthobracon) orbus Papp, 1981

Distribution: England

Notes: NMS, det. Papp, added here

\section{Bracon (Orthobracon) picticornis Wesmael, 1838}

\section{Nomenclature:}

gallarum Ratzeburg, 1852

versicolor Szépligeti, 1901

ratzeburgensis Strand, 1928

laevigatus Fahringer, 1927 preocc.

Distribution: England, Ireland

\section{Bracon (Orthobracon) procerus Papp, 1965}

Distribution: England

Notes: NMS, det. Papp, added here 
Bracon (Orthobracon) pulcher Bengtsson, 1924

Nomenclature:

bengtssoni Fahringer, 1927

Distribution: England

Notes: NMS, det. Papp, added here

Bracon (Orthobracon) roberti (Wesmael, 1838)

Nomenclature:

Braco roberti Wesmael, 1838

Distribution: England, Ireland

Bracon (Orthobracon) romani Fahringer, 1927

Distribution: England, Scotland, Wales, Ireland

Notes: NMS, det. Papp, added here

Bracon (Orthobracon) subcylindricus (Wesmael, 1838)

Nomenclature:

Braco subcylindricus Wesmael, 1838

niger (Vojnovskaja-Krieger, 1929, Baryproctus)

Distribution: England

\section{Bracon (Orthobracon) subsinuatus Szépligeti, 1901}

Distribution: England, Scotland, Ireland

Notes: Added by Papp (2008a). Removed from synonymy with epitriptus by Papp (2008a), who treats both species as belonging to the subgenus Glabrobracon, contrary to their placement in Taxapad (Yu et al. 2012).

Bracon (Orthobracon) titubans (Wesmael, 1838)

Nomenclature:

Braco titubans Wesmael, 1838

tarsator Thomson, 1892

terebrator Szépligeti, 1901

Distribution: England 
Notes: NMS, BMNH, det. Papp, added here

Bracon (Orthobracon) virgatus Marshall, 1897

Nomenclature:

lineifer van Achterberg, 1988; synonymy by Papp (1999c)

Distribution: England, Scotland

Notes: some distribution data from Shaw and Bailey (1991)

Subgenus Osculobracon Papp, 2008

\section{Bracon (Osculobracon) osculator Nees, 1811}

Nomenclature:

bisignatus (Wesmael, 1838, Braco)

degenerator Marshall, 1885

minutus Szépligeti, 1901

temporalis Telenga, 1936

venustus Telenga, 1936

Distribution: England, Scotland, Wales, Ireland

\section{Subgenus Pigeria van Achterberg, 1985}

Notes: Described as a separate genus but Quicke and Sharkey (1989) suggested that

Pigeria could be treated as a subgenus ofBracon, which was followed by Papp (1998).

\section{Bracon (Pigeria) piger (Wesmael, 1838)}

\section{Nomenclature:}

Braco piger Wesmael, 1838

rotundatus Szépligeti, 1901; synonymy by Papp (2008a)

rotundulus Szépligeti, 1904

Distribution: England, Ireland

Notes: Bracon semiluteus Walker, 1874 is not a synonym of piger (contra Morley (1913) and subsequent authors) but is a junior synonym of another species of Bracon (Papp, in prep.). 


\section{Bracon (Pigeria) wolschrijni (van Achterberg, 1985)}

Nomenclature:

Pigeria wolschrijni van Achterberg, 1985

Distribution: Wales

Notes: NMS, det. van Achterberg, added here

\section{Subgenus Rostrobracon Tobias, 1957}

Notes: species of Bracon (Rostrobracon) excluded from the British and Irish list:

[urinator (Fabricius, 1798, Ichneumon); syn. cuspidator (Rossi, 1792, Ichneumon); comptus Marshall, 1897] Marshall (1900) mentions an English specimen but this seems unlikely in view of its present southern European range.

Tribe Coeloidini Tobias, 1957

\section{Genus Coeloides Wesmael, 1838}

Nomenclature:

SYNTOMOMELUS Kokujev, 1902

HABROBRACONIDEA Viereck, 1912

COELOIDINA Viereck, 1921

CEROBRACON Viereck, 1926

Notes: Distribution data from Shaw (2000b).

\section{Coeloides abdominalis (Zetterstedt, 1838)}

Nomenclature:

Bracon abdominalis Zetterstedt, 1838

Distribution: England

\section{Coeloides filiformis Ratzeburg, 1852}

Nomenclature:

melanurus Ivanov, 1896

Distribution: England

Notes: added by Shaw (2000b) 
Coeloides melanostigma Strand, 1918

Nomenclature:

sordidator misident.

stigmaticus Hellén, 1928

Distribution: England

Notes: Added by Shaw (2000b); according to Haeselbarth (in Belokobylskij et al. 2003), the name sordidator (Ratzeburg, 1844, Bracon) probably does not belong in Coeloides, so the species usually referred to as sordidator takes the name melanostigma.

Coeloides melanotus Wesmael, 1838

Nomenclature:

flavus Ivanov, 1896

maculatus Ivanov, 1896

Distribution: England, Wales

Coeloides scolyticida Wesmael, 1838

Nomenclature:

initiatellus (Ratzeburg, 1848, Bracon)

Distribution: England

\section{Cardiochilinae}

\section{Subfamily Cardiochilinae Ashmead, 1900}

Notes: no British or Irish species

\section{Genus Cardiochiles Nees, 1819}

Nomenclature:

Ditherus Cameron, 1902

Notes: species of Cardiochiles excluded from the British and Irish list:

[saltator (Fabricius, 1781, Ichneumon); syn. brachialis Rondani, 1877; katkowi Kokujev, 1895; fumipennis Szépligeti, 1901; sibiricus Telenga, 1955] not British, recorded in error (Shaw and Huddleston 1991) 


\section{Cenocoeliinae}

Subfamily Cenocoeliinae Szépligeti, 1901

Notes: Distribution data from Shaw (1999).

\section{Genus Cenocoelius Haliday, 1840}

Nomenclature:

LACCOPHRYS Förster, 1863

PROMACHUS Cresson, 1887 preocc.

CAENOCOELIUS Marshall, 1894

POSTPROMACHUS Maes, 1999

\section{Cenocoelius aartseni (van Achterberg, 1994)}

Nomenclature:

Promachus aartseni van Achterberg, 1994

Distribution: England

Notes: added by Shaw (1999)

\section{Cenocoelius analis (Nees, 1834)}

Nomenclature:

Bracon analis Nees, 1834

flavifrons Haliday, 1840

cephalotes (Ratzeburg, 1848, Opius) preocc.

magdalini (Förster, 1863, Laccophrys)

hungaricus Kiss, 1927

Distribution: England, Ireland

Genus Lestricus Reinhard, 1865

Lestricus secalis (Linnaeus, 1758)

Nomenclature:

Ichneumon secalis Linnaeus, 1758

agricolator (Linnaeus, 1767, Ichneumon) 
rubriceps (Ratzeburg, 1844, Alysia)

femorator (Tobias, 1973, Cenocoelius)

Distribution: Scotland

Notes: NMS, det. Shaw, added here; removed from the British list by Shaw (1999) but recently found in Scotland.

\section{Charmontinae}

Subfamily Charmontinae van Achterberg, 1979

\section{Genus Charmon Haliday, 1833}

Nomenclature:

PROVANCHERIA Ashmead, 1900

CYCLOCORMUS Cameron, 1911

EUBADIZON misident.

\section{Charmon cruentatus Haliday, 1833}

Nomenclature:

$$
\begin{aligned}
& \text { pectoralis (Nees, 1834, Eubadizon) } \\
& \text { pleuralis (Cresson, 1872, Eubadizon) } \\
& \text { luteus (Cameron, 1911, Cyclocormus) } \\
& \text { ?brevicauda (Hellén, 1958, Eubadizon) }
\end{aligned}
$$

Distribution: England, Scotland, Wales, Ireland, Isle of Man

Notes: Although Achterberg (1979) treated brevicauda as a 'form' of cruentatus, the possibility of a separate species requires investigation.

\section{Charmon extensor (Linnaeus, 1758)}

\section{Nomenclature:}

Ichneumon extensor Linnaeus, 1758

gracilis (Provancher, 1880, Eubadizon)

hungaricus (Kiss, 1927, Calyptus)

striatus (Shestakov, 1940, Eubadizon)

Distribution: England, Scotland, Ireland 


\section{Cheloninae}

\section{Subfamily Cheloninae Förster, 1863}

Nomenclature:

ADELIINAE Viereck, 1918

ACAELIINAE Viereck, 1918

ACOELIINAE Viereck, 1918

Notes: The genus Adelius and related extralimital genera have usually been treated as comprising a separate subfamily, Adeliinae. Recent phylogenetic studies (Belshaw et al. 2000, Belshaw and Quicke 2002) have placed the adeliines within the Cheloninae, as sister taxon to Phanerotoma.

\section{Tribe Chelonini Förster, 1863}

\section{Genus Ascogaster Wesmael, 1835}

Nomenclature:

CASCOGASTER Baker, 1926

LEPTODREPANA Shaw, 1983; synonymy by Achterberg (1990)

Notes: Distribution and synonymic data taken from Huddleston (1984).

Species of Ascogster excluded from the British and Irish list:

[bicarinata (Herrich-Schäffer, 1838, Chelonus); syn. mlokossewitschi Kokujev, 1895; syn. rufiventris Telenga, 1941; preocc.] Included by Huddleston (1978) but no British specimens were seen by Huddleston (1984).

[similis (Nees, 1816, Chelonus)] The type is lost and the species is unplaceable Huddleston (1984).

\section{Ascogaster abdominator (Dahlbom, 1833)}

Nomenclature:

Chelonus abdominator Dahlbom, 1833

instabilis Wesmael, 1835

fulviventris Curtis, 1837

femoralis (Herrich-Schäffer, 1838, Chelonus)

rufiventris (Herrich-Schäffer, 1838, Chelonus)

pallida Ruthe, 1855 
Distribution: England, Scotland, Ireland

\section{Ascogaster albitarsus Reinhard, 1867}

Nomenclature:

similis (Herrich-Schäffer, 1838, Chelonus)

leptopus Thomson, 1874

Distribution: Scotland, Ireland

Notes: added by Huddleston (1984)

\section{Ascogaster annularis (Nees, 1816)}

Nomenclature:

Sigalphus annularis Nees, 1816

Distribution: England

\section{Ascogaster armata Wesmael, 1835}

Nomenclature:

pulchella (Curtis, 1829, Chelonus) nom. nud.

esenbeckii Curtis, 1837

luteicornis (Herrich-Schäffer, 1838, Chelonus)

Distribution: England, Wales

\section{Ascogaster bidentula Wesmael, 1835}

Nomenclature:

scabriuscula (Zetterstedt, 1838, Sigalphus)

multiarticulata (Ratzeburg, 1852, Chelonus)

gibbiscuta Thomson, 1874

fuscipennis Thomson, 1892

atamiensis Ashmead, 1906

Distribution: England, Scotland, Wales, Ireland

Notes: added by Huddleston (1984) 
Ascogaster brevicornis Wesmael, 1835

Nomenclature:

monilicornis (Herrich-Schäffer, 1838, Chelonus)

Distribution: England, Ireland

Notes: added by Huddleston (1984)

Ascogaster canifrons Wesmael, 1835

Nomenclature:

graniger Thomson, 1892

zernyana Fahringer, 1925

Distribution: England, Ireland

Ascogaster consobrina Curtis, 1837

Distribution: England, Scotland, Ireland

Ascogaster dentifer Tobias, 1976

Distribution: England

Notes: added by Huddleston (1984)

Ascogaster dispar Fahringer, 1934

Nomenclature:

spinifer Tobias, 1964

kozlovi Tobias, 1972

Distribution: England

Notes: added by Huddleston (1984)

Ascogaster gonocephala Wesmael, 1835

Distribution: England

Notes: added by Huddleston (1984) 
Ascogaster grahami Huddleston, 1984

Distribution: England

Notes: added by Huddleston (1984)

Ascogaster klugii (Nees, 1816)

Nomenclature:

Sigalphus klugii Nees, 1816

ruficeps Wesmael, 1835

neesii Reinhard, 1867

Distribution: England

Notes: added by Huddleston (1984)

\section{Ascogaster quadridentata Wesmael, 1835}

Nomenclature:

pallidicornis Curtis, 1837

impressa (Herrich-Schäffer, 1838, Chelonus)

quadridens (Herrich-Schäffer, 1838, Chelonus)

cynipum Thomson, 1892

nigricornis Thomson, 1892

egregia Kokujev, 1895

nigrator (Szépligeti, 1896, Chelonus)

carpocapsae (Viereck, 1909, Chelonus)

epinotiae Watanabe, 1937

Distribution: England

\section{Ascogaster rufidens Wesmael, 1835}

Nomenclature:

rufipes (Herrich-Schäffer, 1838, Chelonus) preocc.

laevigator (Ratzeburg, 1852, Chelonus)

Distribution: England, Ireland 
Ascogaster rufipes (Latreille, 1809)

Nomenclature:

Sigalphus rufipes Latreille, 1809

elegans (Nees, 1816, Sigalphus)

fasciata (Dahlbom, 1833, Chelonus)

pallipes (Herrich-Schäffer, 1838, Chelonus)

rubripes (Lucas, 1849, Chelonus)

rugosula (Goureau, 1861, Chelonus)

ratzeburgii Marshall, 1885

arisanica Sonan, 1932

nigribasis Fahringer, 1934

soror Telenga, 1941

Distribution: England, Ireland

\section{Ascogaster varipes Wesmael, 1835}

Nomenclature:

atriceps (Ratzeburg, 1844, Chelonus)

tersa Reinhard, 1867

cavifrons Thomson, 1874

sternalis Thomson, 1874

catula (Marshall, 1885, Chelonus); synonymy by Papp (1996c)

jaroslawensis Kokujev, 1895

Distribution: England, Scotland, Ireland

Genus Chelonus Jurine, 1801

Subgenus Chelonus Jurine, 1801

Nomenclature:

Anomala von Block, 1799 nom. ob.

DAVISANIA La Munyon, 1877

ARICHELONUS Viereck, 1913

MEGACHELONUS Baker, 1926 
Chelonus (Chelonus) acuminatus Herrich-Schäffer, 1838

Distribution: England

Notes: NMS, det. Huddleston, added here

Chelonus (Chelonus) annulatus (Nees, 1816)

Nomenclature:

Sigalphus annulatus Nees, 1816

maculatus Szépligeti, 1896

Distribution: England, Scotland, Ireland

Chelonus (Chelonus) asiaticus Telenga, 1941

Distribution: England, Ireland

Notes: BMNH, det. Lozan, added here. The name is a junior homonym of Chelonus asiaticus Fahringer, 1932.

Chelonus (Chelonus) canescens Wesmael, 1835

Distribution: England

Chelonus (Chelonus) carbonator Marshall, 1885

Nomenclature:

asiaticus Fahringer, 1932

Distribution: England

Chelonus (Chelonus) corvulus Marshall, 1885

Nomenclature:

suturatus Szépligeti, 1898

Distribution: England, Scotland

Chelonus (Chelonus) cylindrus (Klug, 1816)

Nomenclature:

Sigalphus cylindrus Klug, 1816

variabilis Herrich-Schäffer, 1838

macrocerus Thomson, 1874 
speculator Marshall, 1885

ebeninus Fahringer, 1934

Distribution: England

Notes: Chelonus speculator was listed as a synonym of oculator by Huddleston (1978).

Chelonus (Chelonus) decorus Marshall, 1885

Nomenclature:

clavipes Curtis, 1837 nom. nud.

clavipes Fahringer, 1934

szepligetii Fahringer, 1934

Distribution: England

Chelonus (Chelonus) inanitus (Linnaeus, 1767)

Nomenclature:

Cynips inanita Linnaeus, 1767

binarius (Fourcroy, 1785, Ichneumon)

atomos (Rossi, 1790, Ichneumon)

Distribution: England, Ireland

Chelonus (Chelonus) obscuratus Herrich-Schäffer, 1838

Nomenclature:

intermedius Thomson, 1874

Distribution: England

Notes: NMS, det. Huddleston, added here

Chelonus (Chelonus) oculator (Fabricius, 1785)

Nomenclature:

Ichneumon oculator Fabricius, 1785

integer (von Block, 1799, Anomala)

mutabilis (Nees, 1816, Sigalphus)

oculatus (Nees, 1816, Sigalphus)

Distribution: England, Scotland 
Chelonus (Chelonus) pusio Marshall, 1885

Distribution: England

Chelonus (Chelonus) scabrator (Fabricius, 1793)

Nomenclature:

Ichneumon scabrator Fabricius, 1793

scaber (Nees, 1816, Sigalphus)

buccatus Thomson, 1874

Distribution: England

Chelonus (Chelonus) submuticus Wesmael, 1835

Nomenclature:

luteipes Thomson, 1874; synonymy by Papp (1995b)

Distribution: England

Chelonus (Chelonus) wesmaelii Curtis, 1837

Nomenclature:

zimini Tobias, 1972

Distribution: England

Subgenus Microchelonus Szépligeti, 1908

Nomenclature:

CHELONELLA Szépligeti, 1908

NEOCHELONELLA Hincks, 1943

Notes: Treated as a valid genus by Papp (1996c) and Belokobylskij et al. (2003), sometimes treated as a straight synonym of Chelonus (Achterberg and Polaszek 1996), here treated as a subgenus of Chelonus, following Taxapad (Yu et al. 2012).

Chelonus (Microchelonus) atripes Thomson, 1874

Nomenclature:

cunctator (Papp, 1971, Microchelonus)

kamtshaticus (Tobias, 1986, Microchelonus)

Distribution: England 
Notes: NMS, BMNH, det. Huddleston and Lozan, added here

Chelonus (Microchelonus) basalis Curtis, 1837

Distribution: England, Scotland

Chelonus (Microchelonus) binus (Tobias, 1995)

Nomenclature:

Microchelonus binus Tobias, 1995

Distribution: England

Notes: BMNH, det. Lozan, added here

Chelonus (Microchelonus) contractus (Nees, 1816)

Nomenclature:

Sigalphus contractus Nees, 1816

compressiscapus Szépligeti, 1898; synonymy by Papp (1996c)

Distribution: England, Scotland, Wales, Ireland

Notes: some distribution data from Tobias and Shaw (2005)

Chelonus (Microchelonus) depressus Thomson, 1874

Distribution: England, Scotland, Wales

Notes: NMS, BMNH, det. Huddleston and Lozan, added here

Chelonus (Microchelonus) exilis Marshall, 1885

Nomenclature:

excavatus Tobias, 1972; synonymy by Papp (1995b)

Distribution: England

Chelonus (Microchelonus) fenestratus (Nees, 1816)

Nomenclature:

Sigalphus fenestratus Nees, 1816

dispar Marshall, 1885

bimaculatus Ivanov, 1899 preocc. 
Distribution: England

Notes: Synonymised under contractus by Papp (1995b) but treated as a separate species by Tobias and Lozan (2003) and listed as such in Taxapad (Yu et al. 2012).

\section{Chelonus (Microchelonus) fumipennis (Tobias, 1986)}

Nomenclature:

Microchelonus fumipennis Tobias, 1986

Notes: BMNH, det. Lozan, added here; specimen lacking locality data, Billups coll.

\section{Chelonus (Microchelonus) latrunculus Marshall, 1885}

Nomenclature:

?parcicornis Herrich-Schäffer, 1838

thomsonii Dalla Torre, 1898

polonicus Fahringer, 1934

rectus (Papp, 1971, Microchelonus)

Distribution: England, Scotland

Notes: Although parcicornis would have priority, Papp (1995b) states that the type is lost and its identity unverifiable.

\section{Chelonus (Microchelonus) lugubris Wesmael, 1835}

Distribution: England, Ireland

Notes: NMS, BMNH, det. Huddleston and Lozan, added here

\section{Chelonus (Microchelonus) microphtalmus Wesmael, 1838}

Nomenclature:

dilatus Papp, 1971

Distribution: England

Notes: NMS, det. Huddleston, added here

Chelonus (Microchelonus) miscellae (Tobias \& Shaw, 2005)

Nomenclature:

Microchelonus miscellae Tobias \& Shaw, 2005

Distribution: England 
Notes: added by Tobias and Shaw (2005)

Chelonus (Microchelonus) retusus (Nees, 1816)

Nomenclature:

Sigalphus retusus Nees, 1816

emarginatus Herrich-Schäffer, 1838

subemarginatus Herrich-Schäffer, 1838

caudatus Thomson, 1874

pamiricus Vojnovskaja-Krieger, 1931

Distribution: England

Notes: BMNH, det. Lozan, added here

Chelonus (Microchelonus) risorius Reinhard, 1867

Nomenclature:

fissus Szépligeti, 1900 preocc.

fissuralis (Tobias, 1964, Neochelonella)

magnifissus (Tobias, 1986, Microchelonus)

Distribution: England

Chelonus (Microchelonus) sulcatus Jurine, 1807

Distribution: England

Subgenus Parachelonus Tobias, 1995

Chelonus (Parachelonus) gravenhorstii (Nees, 1816)

Nomenclature:

Sigalphus gravenhorstii Nees, 1816

maculator Dahlbom, 1833

eurytheca Wesmael, 1838

adjaricus Tobias, 1976

tricolor Tobias, 1976

Distribution: England 
Chelonus (Parachelonus) pellucens (Nees, 1816)

Nomenclature:

Sigalphus pellucens Nees, 1816

nitens Reinhard, 1867

alboannulatus Szépligeti, 1896

pulchricornis Szépligeti, 1898

varimaculatus Tobias, 1986

austriacus Fahringer, 1934

Distribution: England

Notes: added by Papp (2004c)

Subgenus Stylochelonus Hellén, 1958

Chelonus (Stylochelonus) pedator Dahlbom, 1833

Nomenclature:

secutor (Marshall, 1885, Chelonus)

Distribution: England, Scotland

Chelonus (Stylochelonus) pusillus (Szépligeti, 1908)

Nomenclature:

Stylochelonus pusillus Szépligeti, 1908

furtivus (Tobias, 1986, Microchelonus)

tuberculiventris (Tobias, 1986, Microchelonus)

Distribution: England

Notes: BMNH, det. Lozan, added here

Tribe Phanerotomini Baker, 1926

Genus Adelius Haliday, 1833

Nomenclature:

ACAELIUS Haliday, 1834

ACOELIUS Haliday, 1835

PLEIOMERUS Wesmael, 1837 
ANOMOPTERUS Rohwer, 1914

MYRIOLA Shestakov, 1932

\section{Adelius erythronotus (Förster, 1851)}

Nomenclature:

Acoelius erythronotus Förster, 1851

pyrrhia (Beirne, 1945, Acoelius)

flavus (Tobias, 1966, Acoelius)

Distribution: England, Ireland

Notes: Adelius pyrrhia was listed as a valid species by O'Connor et al. (1999) but as a synonym of erythronotus by Belokobylskij et al. (2003).

\section{Adelius germanus (Haliday, 1834)}

Nomenclature:

Acaelius germanus Haliday, 1834

Distribution: England, Ireland

\section{Adelius subfasciatus Haliday, 1833}

Nomenclature:

minutissimus (Zetterstedt, 1840, Bracon)

parvulus (Förster, 1851, Acoelius)

Distribution: England, Wales, Ireland

Notes: some distribution data from Shaw and Askew (1976)

\section{Adelius viator (Förster, 1851)}

Nomenclature:

Acoelius viator Förster, 1851

Distribution: England 
Genus Phanerotoma Wesmael, 1838

Subgenus Phanerotoma Wesmael, 1838

Nomenclature:

PHANEROGASTER Wesmael, 1838 unavailable

SULYDUS Du Buysson, 1897

ICHNEUTIPTERUS Vachal, 1907; synonymy by Achterberg (1990)

NEOPHANEROTOMA Szépligeti, 1908

NEOACAMPSIS Szépligeti, 1914; synonymy by Achterberg (1990)

PHANEROTOMINA Shestakov, 1930

Notes: species of Phanerotoma (Phanerotoma) excluded from the British and Irish list:

[planifrons (Nees, 1816, Sigalphus); syn. blanda Fahringer, 1934; bicolor Snoflák, 1958 preocc.; asini Llopis Minguez, 1968; snoflaki Shenefelt, 1973] Prior to van Achterberg's (Achterberg 1990) revision, dentata had requently been misidentified as planifrons. All of the British specimens identified as planifrons in the BMNH are in fact dentata.

Phanerotoma (Phanerotoma) acuminata Szépligeti, 1908

Distribution: England

Notes: added by Achterberg (1990)

Phanerotoma (Phanerotoma) dentata (Panzer, 1805)

Nomenclature:

Chelonus dentatus Panzer, 1805

dentator (Nees, 1816, Sigalphus)

rendilea Fahringer, 1934

minor Šnoflák, 1951; synonymy by Achterberg (1990)

Distribution: England

Notes: Huddleston (1978) listed Sigalphus rufescens Latreille, 1809 as a junior synonym of dentata but this is now considered to be a separate species, not occurring in Britain or Ireland (Achterberg 1990), with historical records being misidentifications. 
Phanerotoma (Phanerotoma) leucobasis Kriechbaumer, 1894

Nomenclature:

ocularis Kohl, 1906

ornatulopsis de Saeger, 1942

desertorum Hedwig, 1957; synonymy by Achterberg (1990)

flavitestacea Fischer, 1939; synonymy by Achterberg (1990)

caboverdensis Hedqvist, 1965

Distribution: England

Notes: Added by van Achterberg (1990). Phanerotoma rjabovi Vojnovskaja-Krieger, 1929 and media Shestakov, 1930, synonymised under leucobasis by Achterberg (1990), are now considered to be junior synonyms of fracta Kokujev, 1903 (Belokobylskij 2000b).

\section{Subgenus Bracotritoma Csiki, 1909}

Nomenclature:

TRITOMA Szépligeti, 1908 preocc.; synonymy by Achterberg (1990)

SZEPLIGETIA Schulz, 1911; synonymy by Achterberg (1990)

TRITOMIOS Strand, 1921; synonymy by Achterberg (1990)

UNICA Šnoflák, 1951

\section{Phanerotoma (Bracotritoma) bilinea Lyle, 1924}

Nomenclature:

gregori Šnoflák, 1951

Distribution: England

Phanerotoma (Bracotritoma) tritoma (Marshall, 1898)

Nomenclature:

Chelonus tritomus Marshall, 1898

antennalis Šnoflák, 1951; synonymy by Achterberg (1990)

Distribution: England, Wales, Ireland 


\section{Doryctinae}

\section{Subfamily Doryctinae Förster, 1863}

Notes: Tribal classification follows Belokobylskij (1992), but note that recent work suggests that none of the major doryctine tribes are actually monophyletic (Belokobylskij et al. 2004). Generic synonyms for the most part follow Belokobylskij et al. (2004).

\section{Tribe Doryctini Förster, 1863}

Genus Dendrosoter Wesmael, 1838

Nomenclature:

EURYBOLUS Ratzeburg, 1848

CAENOPACHYS Förster, 1863

Notes: Although Mancini et al. (2003) argued that Caenopachys should be treated as a separate genus, Gebiola et al. (2015) presented molecular phylogenetic evidence that that 'Caenopachys' species (including hartigii in Britain) are nested within Dendrosoter.

\section{Dendrosoter hartigii (Ratzeburg, 1848)}

Nomenclature:

Bracon hartigii Ratzeburg, 1848

flaviventris Förster, 1878

caenopachoides Ruschka, 1925; synonymy by Gebiola et al. (2015)

hartigi misspelling

Distribution: England

\section{Dendrosoter middendorffi (Ratzeburg, 1848)}

Nomenclature:

Bracon middendorffi Ratzeburg, 1848

schimitscheki Fahringer, 1941

Distribution: England

Notes: NMS, det. Shaw, added in Fauna Europaea 
Dendrosoter protuberans (Nees, 1834)

Nomenclature:

Bracon protuberans Nees, 1834

insignis Förster, 1878

Distribution: England

Genus Doryctes Haliday, 1836

Nomenclature:

ISCHIOGONUS Wesmael, 1838

Neodoryctes Szépligeti, 1914

UDAMOLCUS Enderlein, 1920

PRISTODORYCTES Kieffer, 1921

Paradoryctes Granger, 1949

PLYCTES Fischer, 1970

Doryctes heydenii Reinhard, 1865

Distribution: England

Doryctes leucogaster (Nees, 1834)

Nomenclature:

Bracon leucogaster Nees, 1834

Notes: \#Probably occurred only in imported timber. Its British status appears to depend solely on specimens reared in July 1908 from Austrian oak imported to a timber yard near Millwall docks (Elliot and Morley 1911).

Doryctes obliteratus (Nees, 1834)

Nomenclature:

Bracon obliteratus Nees, 1834

mutillator misident.

tabidus (Haliday, 1836, Rogas)

brachyurus Marshall, 1888

strigatus Kokujev, 1900

petrovskii Kokujev, 1902

Distribution: England 
Notes: Regarded as a synonym of striatellus by Belokobylskij et al. (2003).

Doryctes pomarius Reinhard, 1865

Nomenclature:

schimitscheki Fahringer, 1931

Distribution: England

Doryctes rossicus Telenga, 1941

Distribution: England

Notes: NMS, det. Shaw, added in Fauna Europaea

Doryctes striatellus (Nees, 1834)

Nomenclature:

Bracon striatellus Nees, 1834

maculipes Curtis, 1837 nom. nud.

disparator (Ratzeburg, 1844, Bracon)

rex Marshall, 1897

striatelloides Strand, 1918

yogoi Watanabe, 1954

ambigua Kokujev, 1900

notatus Kokujev, 1900

Distribution: England

Doryctes undulatus (Ratzeburg, 1852)

Nomenclature:

Bracon undulatus Ratzeburg, 1852

Distribution: England

\section{Genus Gildoria Hedqvist, 1974}

Notes: Removed from synonymy with Dendrosotinus Telenga, 1941 by Achterberg (2003b). 


\section{Gildoria similis (Bouček, 1955)}

Nomenclature:

Dendrosotinus similis Bouček, 1955

Distribution: England

Notes: added by Shaw (1998)

\section{Genus Ontsira Cameron, 1900}

\section{Nomenclature:}

DORYCTODES Hellén, 1927

Notes: Zaldívar-Riverón et al. (2008a) did not recover Ontsira as monophyletic and consequently some authors (e.g. Achterberg 2014) prefer to treat these species as belonging to Doryctodes.

\section{Ontsira antica (Wollaston, 1858)}

Nomenclature:

Clinocentrus anticus Wollaston, 1858

gallica (Reinhard, 1865, Doryctes)

truncorum (Goureau, 1866, Bracon)

incerta (Ashmead, 1888, Doryctes)

caudalis (Hellén, 1957, Oncophanes)

Distribution: Scotland

\section{Ontsira ignea (Ratzeburg, 1852)}

Nomenclature:

Bracon igneus Ratzeburg, 1852

Distribution: England

Notes: NMS, det. Shaw and van Achterberg, added here

\section{Ontsira imperator (Haliday, 1836)}

\section{Nomenclature:}

Rogas imperator Haliday, 1836

zonata (Wesmael, 1838, Ischiogonus)

praecisa (Ratzeburg, 1852, Bracon) 
cingulata (Provancher, 1880, Syngaster)

dubia (Kokujev, 1900, Doryctes)

iranica (Telenga, 1941, Doryctodes)

niger (Hedwig, 1957, Coeloides)

Distribution: England, Ireland

\section{Genus Rhaconotus Ruthe, 1854}

Nomenclature:

HEDYSOMUS Förster, 1863

HORMIOPTERUS Giraud, 1869

RHADINOGASTER Szépligeti, 1908

EURYPHRYMNUS Cameron, 1910

RHACONOTINUS Hedqvist, 1965

\section{Rhaconotus aciculatus Ruthe, 1854}

Nomenclature:

cerdai Docavo Alberti, 1960

major Tobias, 1964

Distribution: England

Notes: added by Shaw (1998)

\section{Genus Wachsmannia Szépligeti, 1900}

Notes: Belokobylskij (1992) synonymised Wachsmannia under Ontsira but van Achterberg (1995) disagreed and synonymised Wachsmannia under Hypodoryctes Kokujev, 1900. Van Achterberg (pers. comm. and in Fauna Europaea) now regards Wachsmannia as a separate genus again.

\section{Wachsmannia spathiiformis (Ratzeburg, 1848)}

Nomenclature:

Bracon spathiiformis Ratzeburg, 1848

maculipennis Szépligeti, 1900; synonymy by Achterberg (1995)

obliteratus misident.

Distribution: England 
Tribe Ecphylini Hellén, 1957

Genus Ecphylus Förster, 1863

Nomenclature:

TERENUSA Marshall, 1885

PARAECPHYLUS Ashmead, 1900

SACTOPUS Ashmead, 1900

Notes: Other species are treated as synonyms of silesiacus by Belokobylskij et al. (2003) but van Achterberg (in Belokobylskij et al. 2003) states that there is biological evidence for several species.

\section{Ecphylus eccoptogastri (Ratzeburg, 1848)}

Nomenclature:

Bracon eccoptogastri Ratzeburg, 1848

Ecphylus hylesini (Ratzeburg, 1852)

Nomenclature:

Bracon hylesini Ratzeburg, 1852

Distribution: England

Notes: NMS, det. Shaw and van Achterberg, added in Fauna Europaea

\section{Ecphylus pinicola Hedqvist, 1967}

Distribution: England, Scotland

Notes: NMS, det. Shaw and van Achterberg, added in Fauna Europaea

\section{Ecphylus silesiacus (Ratzeburg, 1848)}

Nomenclature:

Bracon silesiacus Ratzeburg, 1848

minutissimus (Ratzeburg, 1848, Bracon) preocc.

Distribution: England

Notes: NMS, det. Shaw and van Achterberg, added in Fauna Europaea; Shaw (1998) recorded silesiacus as British but was using the name in the broader sense (i.e. = eccoptogastri, =hylesini, =pinicola). Listed in Huddleston (1978) as eccoptogastri. 
Tribe Hecabolini Förster, 1863

Genus Hecabolus Curtis, 1834

Nomenclature:

ANISOPELMA Wesmael, 1838

Hecabolus sulcatus Curtis, 1834

Nomenclature:

belgicus (Wesmael, 1838, Anisopelma)

Distribution: England, Wales

\section{Genus Monolexis Förster, 1863}

Notes: Species of Monolexis excluded from the British and Irish list:

[fuscicornis Förster, 1863; syn. lycti (Cresson, 1880, Anisopelma); minimus (Cresson, 1880, Anisopelma); utilis (Cresson, 1880, Anisopelma); doderoi (Mantero, 1910, Hecabolus); lavagnei Picard, 1913; atis Nixon, 1943] Seems to have been included on the British list only on the basis of specimens emerging from Oak (Quercus) timber imported from the USA (e.g. Laing 1928).

\section{Genus Heterospilus Haliday, 1836}

Nomenclature:

SYNODUS misident.

TELEBOLUS Marshall, 1888

KAREBA Cameron, 1905

ANOCATOSTIGMA Enderlein, 1920

HARPAGOLACCUS Enderlein, 1920

LITUANIA Jakimavičius, 1968

Notes: species of Heterospilus excluded from the British and Irish list:

[caesus (Nees, 1834, Bracon) misident.] Excluded by Shaw and Huddleston (1991), see also Shaw (1997).

Heterospilus ater Fischer, 1960

Distribution: England

Notes: added by Shaw (1998) 
Heterospilus fuscexilis Shaw, 1997

Distribution: England

Notes: added by Shaw (1997)

Tribe Spathiini Förster, 1863

\section{Genus Spathius Nees, 1818}

Nomenclature:

STENOPHASMUS Smith, 1859

EUSPATHIUS Förster, 1863

PSEUDOSPATHIUS Szépligeti, 1902

RHACOSPATHIUS Cameron, 1905

\section{Spathius brevicaudis Ratzeburg, 1844}

Distribution: England, Wales

Notes: NMS, det. Shaw, added in Fauna Europaea

\section{Spathius exarator (Linnaeus, 1758)}

Nomenclature:

Ichneumon exarator Linnaeus, 1758

formicatus (Linnaeus, 1767, Ichneumon)

mutillarius (Fabricius, 1775, Ichneumon)

mystacatus (Schrank, 1781, Ichneumon)

affinis (Fabricius, 1793, Ichneumon)

immaturus (Gravenhorst, 1807, Ichneumon)

clavatus (Panzer, 1809, Cryptus)

affinator (Thunberg, 1824, Ichneumon)

attenuator (Thunberg, 1824, Ichneumon)

formicator (Thunberg, 1824, Ichneumon) preocc.

mutillator (Thunberg, 1824, Ichneumon)

exannulatus Ratzeburg, 1848

ferrugatus Goureau, 1866

strandi Fahringer, 1930 
breviterebrantus Dutu-Lacatusu, 1956

Distribution: England, Scotland, Wales, Ireland

\section{Spathius pedestris Wesmael, 1838}

Nomenclature:

apterus Wollaston, 1858

maderi Fahringer, 1930

hirtus Hedqvist, 1976

Distribution: England

Notes: Recorded as new to Britain by Perkins and Nixon (1939). Huddleston (1978) listed this as a species in need of confirmation, although, being wingless, it is straightforward to identify. There are additional British specimens in BMNH, World Museum Liverpool, Manchester Museum and Leeds City Museum.

\section{Spathius phymatodis Fischer, 1966}

Distribution: England

Notes: NMS, det. Shaw, added in Fauna Europaea

\section{Spathius rubidus (Rossius, 1794)}

Nomenclature:

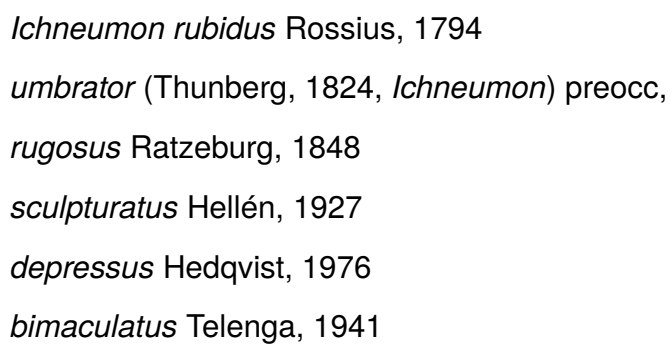

Distribution: England, Scotland, Ireland

\section{Spathius umbratus (Fabricius, 1798)}

\section{Nomenclature:}

Ichneumon umbratus Fabricius, 1798

erythrocephalus Wesmael, 1838

curvicaudis Ratzeburg, 1844 
Distribution: England

Notes: Spathius curvicaudis was recorded as new to Britain by Shaw (1988a) but synonymised under erythrocephalus by Belokobylskij and Samartsev (2014) and Achterberg (2014), who also synonymyised erythrocephalus under umbratus.

\section{Euphorinae}

Subfamily Euphorinae Förster, 1863

Notes: The tribal and generic classification follows Stigenberg et al. (2015).

Tribe Centistini Čapek, 1970

Genus Allurus Förster, 1863

Allurus lituratus (Haliday, 1835)

Nomenclature:

Leiophron lituratus Haliday, 1835

Distribution: Ireland

Notes: Synonymised under muricatus by Achterberg (1985), later removed from synonymy by Achterberg (1997), with a key to the species.

\section{Allurus muricatus (Haliday, 1833)}

Nomenclature:

Ancylus muricatus Haliday, 1833

armatus (Wesmael, 1835, Leiophron)

niger (Lyle, 1926, Leiophron)

Distribution: Scotland, Ireland

Genus Centistes Haliday, 1835

Subgenus Ancylocentrus Förster, 1863

Notes: species of Centistes (Ancylocentrus) excluded from the British and Irish list: 
[subsulcatus (Thomson, 1895, Leiophron)] Not included in Huddleston (1978) but listed as British in Taxapad (Yu et al. 2012) as it was recorded by Kerrich (1932), as a tentative identification (by Roman), which has not been corroborated.

Centistes (Ancylocentrus) ater (Nees, 1834)

Nomenclature:

Leiophron ater Nees, 1834

excrucians (Haliday, 1835, Leiophron)

lativalvis (Jakimavičius, 1972, Allurus)

Distribution: England, Ireland

Centistes (Ancylocentrus) collaris (Thomson, 1895)

Nomenclature:

Leiophron collaris Thomson, 1895

Distribution: England, Ireland

Notes: BMNH, det. Mason, Broad, added here

Centistes (Ancylocentrus) edentatus (Haliday, 1835)

Nomenclature:

Leiophron edentatus Haliday, 1835

Distribution: England, Ireland

Centistes (Ancylocentrus) nasutus (Wesmael, 1838)

Nomenclature:

Eubadizon nasutus Wesmael, 1838

saxo (Reinhard, 1862, Leiophron)

Distribution: England

Notes: some distribution data from Luff (1976a)

\section{Subgenus Centistes Haliday, 1835}

Nomenclature:

ANCYLUS Haliday, 1833

ANCYLLUS Haldeman, 1842 
EUPHORIDEA Ashmead, 1900

LIOSIGALPHUS Ashmead, 1900

Centistes (Centistes) cuspidatus (Haliday, 1833)

Nomenclature:

Ancylus cuspidatus Haliday, 1833

Iucidator (Nees, 1834, Bracon)

Distribution: England, Ireland

Centistes (Centistes) fuscipes (Nees, 1834)

Nomenclature:

Bracon fuscipes Nees, 1834

fuscipes (Wesmael, 1835, Leiophron)

Distribution: England

Subgenus Syrrhizus Förster, 1863

Centistes (Syrrhizus) delusorius (Förster, 1863)

Nomenclature:

Syrrhizus delusorius Förster, 1863

Distribution: England, Scotland

Tribe Cosmophorini Muesebeck \& Walkley, 1951

Genus Cosmophorus Ratzeburg, 1848

Nomenclature:

COSMOPHORINUS Viereck, 1925

Cosmophorus cembrae Ruschka, 1925

Distribution: England

Notes: added by Shaw (1989)

Genus Ropalophorus Curtis, 1837

Nomenclature: 
RHOPALOPHORUS Blanchard, 1840 emendation, preocc.

CORYNOPHORE Blanchard, 1845

EUSTALOCERUS Förster, 1863

\section{Ropalophorus clavicornis (Wesmael, 1835)}

\section{Nomenclature:}

Microctonus clavicornis Wesmael, 1835

wisconsinensis Shenefelt, 1960; synonymy by Yang et al. (2003)

Distribution: England

\section{Tribe Dinocampini Shaw, 1985}

\section{Genus Dinocampus Förster, 1863}

Dinocampus coccinellae (Schrank, 1802)

Nomenclature:

Ichneumon coccinellae Schrank, 1802

terminatus (Nees, 1812, Bracon)

sculptus (Cresson, 1872, Euphorus)

americanus (Riley, 1888, Centistes)

Distribution: England, Scotland, Wales, Ireland

Tribe Euphorini Förster, 1863

\section{Genus Leiophron Nees, 1818}

Notes: Distribution and much taxonomic data from Richards (1967), New (1970) and Loan (1974).

\section{Subgenus Euphorus Nees, 1834}

Notes: Treated as a subgenus of Leiophron by Belokobylskij (2000b).

Leiophron (Euphorus) duploclaviventris Shenefelt, 1969

Nomenclature:

claviventris (Ruthe, 1856, Microctonus) preocc.

ruthei Loan, 1974 
Distribution: England

Leiophron (Euphorus) pallidistigma (Curtis, 1833)

Nomenclature:

Leiophron pallidistigma Curtis, 1833

pallicornis (Nees, 1834, Euphorus)

claviventris (Wesmael, 1835, Microctonus)

intacta (Haliday, 1835, Leiophron)

parvula (Ruthe, 1856, Microctonus)

Distribution: England, Ireland

Leiophron (Euphorus) similis (Curtis, 1833)

Nomenclature:

Leiophron similis Curtis, 1833

basalis (Curtis, 1833, Leiophron)

Distribution: England, Wales, Ireland

Notes: Listed twice, under Microctonus and Leiophron, by Huddleston (1978).

Subgenus Leiophron Nees, 1818

Nomenclature:

Liophron Förster, 1863

Leiophron (Leiophron) apicalis Haliday, 1833

Nomenclature:

apicalis Curtis, 1833 preocc.

ornata (Marshall, 1887, Euphorus)

Distribution: England, Ireland, Isle of Man

Notes: The type of Euphorus ornatus is lost but Loan (1974) suggested, on the basis of possible type material (collected in England), that ornata may be a synonym of apicalis, which has been formally proposed by Papp (2003).

Leiophron (Leiophron) fascipennis (Ruthe, 1856)

Nomenclature:

Microctonus fascipennis Ruthe, 1856 
fasciipennis misspelling

aciculata Belokobylskij, 1993

Distribution: England

Leiophron (Leiophron) fulvipes Curtis, 1833

Distribution: England

Leiophron (Leiophron) heterocordyli Richards, 1967

Distribution: England

\section{Genus Peristenus Förster, 1863}

Notes: species of Peristenus excluded from the British and Irish list:

[brevicornis (Herrich-Schäffer, 1838, Perilitus)] Listed by Loan (1974) as incertae sedis within Peristenus or Leiophron, by Huddleston (1978) as a doubtfully placed species of Leiophron and by van Achterberg (in Belokobylskij et al. 2003) as a species of Peristenus. We cannot trace any references to this species occurring in Britain or Ireland.

[duplobrevicornis (Shenefelt, 1969, Leiophron); syn. brevicornis (Ruthe, 1856, Microctonus) preocc.] According to Loan (1974), the type of brevicornis (Ruthe) is lost and the species cannot be identified with certainty. Recorded as British by Marshall (1872). Belokobylskij et al. (2003) list it as a species of Peristenus (= Leiophron according to Belokobylskij).

[mitis (Haliday, 1833, Leiophron)] According to van Achterberg (in Belokobylskij et al. 2003) the type is lost and was probably a deformed specimen anyway.

\section{Peristenus accinctus (Haliday, 1835)}

Nomenclature:

Leiophron accinctus Haliday, 1835

laeviventris (Ruthe, 1856, Microctonus)

intermedius (Ruthe, 1856, Microctonus)

Peristenus antennalis (Hincks, 1943) preocc.

Nomenclature:

Leiophron antennalis Hincks, 1943

picipes (Haliday, 1835, Leiophron) preocc. 
Distribution: Ireland

Notes: Added by Achterberg (1997). Hincks's was a replacement name for Haliday's use of the name picipes for a species that is distinct from picipes (Curtis). Preoccupied by Leiophron antennalis Watanabe, 1937 (now classified in Centistes (Ancylocentrus)).

Peristenus facialis (Thomson, 1892)

Nomenclature:

Euphorus facialis Thomson, 1892

fascialis misspelling microcerus (Thomson, 1892, Euphorus)

Distribution: England, Wales

\section{Peristenus grandiceps (Thomson, 1892)}

Nomenclature:

Euphorus grandiceps Thomson, 1892

Distribution: England, Ireland

Notes: Not listed by Huddleston (1978), although it was taken out of synonymy with orchesiae by Loan (1974).

Peristenus malatus Loan, 1976

Distribution: Scotland

Notes: distribution data from Loan (1976)

Peristenus nitidus (Curtis, 1833)

Nomenclature:

Leiophron nitidus Curtis, 1833

Distribution: England

\section{Peristenus orchesiae (Curtis, 1833)}

Nomenclature:

Leiophron orchesiae Curtis, 1833

rufibarbis (Curtis, 1837, Leiophron)

Distribution: England 
Peristenus orthotyli (Richards, 1967)

Nomenclature:

Leiophron orthotyli Richards, 1967

Distribution: England

Peristenus pallipes (Curtis, 1833)

Nomenclature:

Leiophron pallipes Curtis, 1833

barbiger (Wesmael, 1835, Microctonus)

Distribution: England, Scotland, Ireland, Isle of Man

Notes: Van Achterberg has identified a Scottish specimen as barbiger, currently listed as a junior synonym of pallipes.

Peristenus pallipes (Herrich-Schäffer, 1838) preocc.

Nomenclature:

Perilitus pallipes Herrich-Schäffer, 1838

mellipes (Cresson, 1872, Euphorus)

punctatus (Provancher, 1883, Microctonus)

tuberculifer (Marshall, 1887, Euphorus)

nocturnus (Viereck, 1905, Brachistes)

Peristenus picipes (Curtis, 1833)

Nomenclature:

Leiophron picipes Curtis, 1833

coactus (Marshall, 1887, Euphorus)

Distribution: England, Ireland

Tribe Helorimorphini Schmiedeknecht, 1907

Genus Chrysopophthorus Goidanich, 1948

Chrysopophthorus hungaricus (Kiss, 1927)

Nomenclature:

Helorimorpha hungaricus Kiss, 1927 
chrysopimaginis Goidanich, 1948

elegans Tobias, 1961

Distribution: England

Notes: added by Shaw (1996a)

Genus Wesmaelia Förster, 1863

Wesmaelia petiolata (Wollaston, 1858)

Nomenclature:

Euphorus petiolatus Wollaston, 1858

pendula Förster, 1863

cremasta Marshall, 1872

americana Myers, 1917

asiatica Shestakov, 1932

Distribution: England

Tribe Myiocephalini Chen \& van Achterberg, 1997

Nomenclature:

LOXOCEPHALINI Shaw, 1985 invalid

Genus Myiocephalus Marshall, 1897

Nomenclature:

LOXOCEPHALUS Förster, 1863 preocc.

SPILOMMA Morley, 1909

Myiocephalus boops (Wesmael, 1835)

Nomenclature:

Microctonus boops Wesmael, 1835

longipes (Förster, 1863, Loxocephalus)

laticeps (Provancher, 1886, Gamosecus)

falconivibrans (Morley, 1909, Spilomma)

hedini (Fahringer, 1930, Aphidius)

Distribution: England, Ireland 
Tribe Neoneurini Bengtsson, 1918

Nomenclature:

ELASMOSOMINI Viereck, 1918

Notes: Treated as a separate subfamily in many works (e.g. Shaw and Huddleston 1991, Achterberg 1993b) but recent phylogenetic studies have shown the neoneurines to be nested within the Euphorinae (e.g. Belshaw and Quicke 2002, Dowton et al. 2002).

Genus Elasmosoma Ruthe, 1858

Nomenclature:

PARAMIRAX Ashmead, 1895 unavailable

Elasmosoma berolinense Ruthe, 1858

Distribution: England

Notes: added by Shaw (2009)

\section{Genus Neoneurus Haliday, 1838}

Nomenclature:

ECCLITES Förster, 1863

SIXIA Vollenhoven, 1867; synonymy by Achterberg (1997)

Neoneurus auctus (Thomson, 1895)

Nomenclature:

Elasmosoma aucta Thomson, 1895

halidaii Marshall, 1897

bistigmaticus (Morley, 1909, Euphorus)

Distribution: England, Scotland

Tribe Perilitini Förster, 1863

Nomenclature:

MICROCTONINI Shaw, 1985 


\section{Genus Microctonus Wesmael, 1835}

Nomenclature:

GAMOSECUS Provancher, 1880

Notes: Haeselbarth (2008) described many new species in Perilitus (Microctonus) but as Microctonus was raised to generic rank by Stigenberg et al. (2015), these are effectively new combinations in Microctonus. Synonymy follows Haeselbarth (2008).

\section{Microctonus aciculatus (Haeselbarth, 2008)}

\section{Nomenclature:}

Perilitus aciculatus Haeselbarth, 2008

Distribution: Ireland

Notes: added by Haeselbarth (2008)

\section{Microctonus aethiops Nees, 1834}

Nomenclature:

spurius Ruthe, 1856

?brevispina (Thomson, 1892, Euphorus)

aethiopoides Loan, 1975

Distribution: England, Ireland

\section{Microctonus alticae (Haeselbarth, 2008)}

Nomenclature:

Perilitus alticae Haeselbarth, 2008

Distribution: England

Notes: added by Haeselbarth (2008)

\section{Microctonus aphthonae (Haeselbarth, 2008)}

Nomenclature:

Perilitus aphthonae Haeselbarth, 2008

Distribution: England, Ireland

Notes: added by Haeselbarth (2008) 
Microctonus apiophaga Loan, 1974

Microctonus areolatus (Thomson, 1892)

Nomenclature:

Perilitus areolatus Thomson, 1892

Distribution: England

Microctonus belokobylskiji (Haeselbarth, 2008)

Nomenclature:

Perilitus belokobylskiji Haeselbarth, 2008

Distribution: Ireland

Notes: added by Haeselbarth (2008)

Microctonus brassicae (Haeselbarth, 2008)

Nomenclature:

Perilitus brassicae Haeselbarth, 2008

Distribution: England

Notes: added by Haeselbarth (2008)

Microctonus brevicollis (Haliday, 1835)

Nomenclature:

Perilitus brevicollis Haliday, 1835

Distribution: England, Ireland

Microctonus cerealium (Haliday, 1835)

Nomenclature:

Perilitus cerealium Haliday, 1835

secalis (Haliday, 1833, Perilitus) unavailable

Distribution: England, Ireland

Microctonus colesi Drea, 1968

Distribution: Scotland, Ireland 
Notes: added by Haeselbarth (2008)

Microctonus consuetor (Nees, 1834)

Nomenclature:

Perilitus consuetor Nees, 1834

Distribution: Scotland

Notes: added by Haeselbarth (2008)

Microctonus debilis (Wollaston, 1858)

Nomenclature:

Perilitus debilis Wollaston, 1858

?gracilipes (Thomson, 1892, Perilitus)

Distribution: Ireland

Notes: added by Haeselbarth (2008)

Microctonus fagi (Haeselbarth, 2008)

Nomenclature:

Perilitus fagi Haeselbarth, 2008

Distribution: England, Ireland

Notes: Added by Haeselbarth (2008); specimens tentatively associated with the type material.

Microctonus fittkaui (Haeselbarth, 2008)

Nomenclature:

Perilitus fittkaui Haeselbarth, 2008

Distribution: England

Notes: added by Haeselbarth (2008)

Microctonus flaviventris (Thomson, 1892)

Nomenclature:

Perilitus flaviventris Thomson, 1892

areolatus (Thomson, 1892, Perilitus) 
Distribution: Ireland

Notes: added by Haeselbarth (2008)

Microctonus haszprunari (Haeselbarth, 2008)

Nomenclature:

Perilitus haszprunari Haeselbarth, 2008

Distribution: Ireland

Notes: added by Haeselbarth (2008)

Microctonus lipari Čapek \& Starý, 1995

Distribution: England

Notes: added by Haeselbarth (2008)

Microctonus melanopus Ruthe, 1856

Distribution: England, Scotland, Ireland

Notes: added by Haeselbarth (2008)

Microctonus parcicornis Ruthe, 1856

Distribution: England

Microctonus perforatus (Haeselbarth, 2008)

Nomenclature:

Perilitus perforatus Haeselbarth, 2008

Distribution: England

Notes: added by Haeselbarth (2008)

Microctonus podargae (Haeselbarth, 2008)

Nomenclature:

Perilitus podargae Haeselbarth, 2008

Distribution: England

Notes: added by Haeselbarth (2008) 
Microctonus retusus Ruthe, 1856

Nomenclature:

lancearius Ruthe, 1856

caudatus (Thomson, 1892, Perilitus)

Distribution: England, Ireland

Notes: Added by Luff (1976b); overlooked by Huddleston (1978) and O'Connor et al. (1999).

Microctonus silvularis (Haeselbarth, 2008)

Nomenclature:

Perilitus silvularis Haeselbarth, 2008

Distribution: Scotland, Ireland

Notes: added by Haeselbarth (2008)

Microctonus stenocari (Haeselbarth, 2008)

Nomenclature:

Perilitus stenocari Haeselbarth, 2008

Distribution: Scotland

Notes: added by Haeselbarth (2008)

Microctonus strophosomi (Haeselbarth, 2008)

Nomenclature:

Perilitus strophosomi Haeselbarth, 2008

Distribution: Scotland, Ireland

Notes: added by Haeselbarth (2008)

Microctonus thyellae (Haeselbarth, 2008)

Nomenclature:

Perilitus thyellae Haeselbarth, 2008

Distribution: England, Scotland

Notes: added by Haeselbarth (2008) 


\section{Genus Perilitus Nees, 1818}

Notes: species of Perilitus excluded from the British and Irish list:

[falciger (Ruthe, 1856, Microctonus)] Richards (1960) states that it is not known with certainty from Britain.

\section{Perilitus areolaris Gerdin \& Hedqvist, 1985}

Distribution: England

Notes: added by Haeselbarth (1999)

Perilitus dubius (Wesmael, 1838)

Nomenclature:

Microctonus dubius Wesmael, 1838

rutilus Herrich-Schäffer, 1838 preocc.

Distribution: England

\section{Perilitus foveolatus Reinhard, 1862}

Nomenclature:

sicheli Giard, 1895; synonymy by Haeselbarth (1999)

Distribution: England, Wales

Perilitus marci Haeselbarth, 1999

Distribution: England

Notes: added by Haeselbarth (1999)

\section{Perilitus rutilus (Nees, 1811)}

Nomenclature:

Bracon rutilus Nees, 1811

luteus Herrich-Schäffer, 1838

ruralis Herrich-Schäffer, 1838

strenuus Marshall, 1887; synonymy by Haeselbarth (1999)

pyri (Viereck, 1917, Dinocampus)

tuberculus Zaykov, 1981; synonymy by Haeselbarth (1999) 
Distribution: England, Ireland

Genus Rilipertus Haeselbarth, 1996

Rilipertus intricatus (Ruthe, 1859)

Nomenclature:

Microctonus intricatus Ruthe, 1859

borealis (Thomson, 1892, Perilitus); synonymy by Haeselbarth (1996)

Distribution: Scotland, Ireland

Genus Spathicopis van Achterberg, 1977

flavocephala van Achterberg, 1977

Distribution: England

Notes: NMS, det. van Achterberg, added here

Tribe Pygostolini Belokobylskij, 2000

Genus Pygostolus Haliday, 1833

Notes: Distribution data from Achterberg (1992a) and BMNH.

Pygostolus falcatus (Nees, 1834)

Nomenclature:

Leiophron falcatus Nees, 1834 testaceus misident.

Distribution: England, Ireland

Pygostolus multiarticulatus (Ratzeburg, 1852)

Nomenclature:

Blacus multiarticulatus Ratzeburg, 1852

falcatus (Wesmael, 1838, Blacus) preocc.

Distribution: England 
Pygostolus otiorhynchi (Boudier, 1834)

Nomenclature:

Bracon otiorhynchi Boudier, 1834

Distribution: England, Scotland

Notes: Achterberg (1992a) separated otiorhynchi from falcatus but Belokobylskij et al. (2003), without comment, treated the two names as synonymous again. This is not followed here as otiorhynchi and falcatus seem to be distinct species.

\section{Pygostolus sticticus (Fabricius, 1798)}

Nomenclature:

Ichneumon sticticus Fabricius, 1798

testaceus (Fallén, 1813, Bassus) preocc.

sticticator (Thunberg, 1824, Ichneumon)

barynoti (Boudier, 1834, Bracon)

gigas (Wesmael, 1835, Blacus)

Distribution: England, Scotland, Ireland, Isle of Man

\section{Tribe Syntretini Shaw, 1985}

\section{Genus Syntretus Förster, 1863}

Nomenclature:

FALCOSYNTRETUS Tobias, 1965

PARASYNTRETUS Belokobylskij, 1993

Notes: Distribution and synonymic data from Achterberg and Haeselbarth (2003).

Species of Syntretus excluded from the British and Irish list:

[parvicornis (Ruthe, 1862, Microctonus)] Not listed as British or Irish by Achterberg and Haeselbarth (2003).

Syntretus breviradialis van Achterberg \& Haeselbarth, 2003

Distribution: England

Notes: added by Broad (2009) 
Syntretus conterminus (Nees, 1834)

Nomenclature:

Perilitus conterminus Nees, 1834

Distribution: England, Scotland, Ireland

Syntretus elegans (Ruthe, 1856)

Nomenclature:

Microctonus elegans Ruthe, 1856

transsylvanicus (Kiss, 1927, Perilitus)

Distribution: Ireland

Notes: added by Achterberg and Haeselbarth (2003)

Syntretus flevo van Achterberg \& Haeselbarth, 2003

Distribution: England, Ireland

Notes: added by Achterberg and Haeselbarth (2003)

Syntretus fuscicoxis van Achterberg \& Haeselbarth, 2003

Distribution: England, Ireland

Notes: added by Achterberg and Haeselbarth (2003)

Syntretus fuscivalvis van Achterberg \& Haeselbarth, 2003

Distribution: England, Scotland, Ireland

Notes: added by Achterberg and Haeselbarth (2003)

Syntretus idalius (Haliday, 1833)

Nomenclature:

Perilitus idalius Haliday, 1833

vernalis (Wesmael, 1835, Microctonus)

cultus (Marshall, 1887, Microctonus)

Distribution: England, Scotland, Ireland 
Syntretus ocularis van Achterberg \& Haeselbarth, 2003

Distribution: England, Wales, Scotland

Notes: added by Achterberg and Haeselbarth (2003)

Syntretus politus (Ruthe, 1856)

Nomenclature:

Microctonus politus Ruthe, 1856

cynthius (Curtis, 1837, Microctonus) nom. nud.

cynthius Lyle, 1927

Distribution: England, Ireland

Syntretus pusio (Marshall, 1898)

Nomenclature:

Microctonus pusio Marshall, 1898

Distribution: England, Ireland

Syntretus splendidus (Marshall, 1887)

Nomenclature:

Microctonus splendidus Marshall, 1887

testaceus (Capron, 1887, Microctonus)

suffolciensis (Morley, 1933, Dyscritus) new synonymy

niger Tobias, 1976

Distribution: England, Scotland, Ireland

Notes: The female holotype of Dyscritus suffolciensis Morley in BMNH, described as an aphidiine, has remained uninterpreted, although Starý (1959) recognised that it is a euphorine. It is a normal specimen of Syntretus splendidus.

Syntretus taegeri van Achterberg \& Haeselbarth, 2003

Distribution: England

Notes: added by Achterberg and Haeselbarth (2003) 
Syntretus xanthocephalus (Marshall, 1887)

Nomenclature:

Microctonus xanthocephalus Marshall, 1887

tempestivus (Curtis, 1837, Microctonus) nom. nud.

lyctaea Cole, 1959

lyctae misspelling

Distribution: England, Scotland, Ireland

Syntretus zuijleni van Achterberg \& Haeselbarth, 2003

Distribution: England, Scotland, Ireland

Notes: added by Achterberg and Haeselbarth (2003)

Tribe Townesilitini Shaw, 1985

Genus Streblocera Westwood, 1833

Notes: Cosmophoridia Hedqvist, 1955 and Eutanycerus, usually considered synonymous with Streblocera, were regarded as valid subgenera by Chen and Achterberg (1997).

Subgenus Streblocera Westwood, 1833

Nomenclature:

LECYTHODELLA Enderlein, 1912

Streblocera (Streblocera) fulviceps Westwood, 1833

Distribution: England

Streblocera (Streblocera) longiscapha Westwood, 1882

Distribution: England

Subgenus Eutanycerus Förster, 1863

Nomenclature:

VILLOCERA Chen \& van Achterberg, 1997; synonymy by Belokobylskij (2000a) 
Streblocera (Eutanycerus) macroscapa (Ruthe, 1856)

Nomenclature:

Microctonus macroscapus Ruthe, 1856

halidayana (Förster, 1863, Eutanycerus)

Distribution: England

Genus Townesilitus Haeselbarth \& Loan, 1983

Townesilitus aemulus (Ruthe, 1856)

Nomenclature:

Microctonus aemulus Ruthe, 1856

punctifrontis (Watanabe, 1955, Microctonus)

Distribution: England, Wales, Ireland

Notes: added by Haeselbarth (1988)

Townesilitus bicolor (Wesmael, 1835)

Nomenclature:

Microctonus bicolor Wesmael, 1835

breviradialis (Tobias, 1976, Microctonus)

Distribution: England, Wales, Ireland

Townesilitus deceptor (Wesmael, 1835)

Nomenclature:

Microctonus deceptor Wesmael, 1835

Distribution: England, Scotland, Ireland

Notes: added by Haeselbarth (1988)

Townesilitus fulviceps (Ruthe, 1856)

Nomenclature:

Microctonus fulviceps Ruthe, 1856

Distribution: England

Notes: added by Haeselbarth (1988) 


\section{Exothecinae}

Subfamily Exothecinae Förster, 1863

Nomenclature:

PHANOMERINAE Fahringer, 1928

\section{Genus Colastes Haliday, 1833}

Notes: Belokobylskij (1998) treats Shawiana and Xenarcha as subgenera of Colastes.

\section{Subgenus Colastes Haliday, 1833}

Nomenclature:

EXOTHECUS Wesmael, 1838

PHANOMERIS Förster, 1863

PHAENOMERIS Dalla Torre, 1898

Colastes (Colastes) affinis (Wesmael, 1838)

Nomenclature:

Exothecus affinis Wesmael, 1838

Distribution: England, Scotland, Wales

Notes: NMS, det. Shaw \& van Achterberg, added here; treated as a species of Colastes (Xenarcha) by Belokobylskij (1998).

\section{Colastes (Colastes) braconius Haliday, 1833}

\section{Nomenclature:}

debilis (Wesmael, 1838, Exothecus)

gracilis Papp, 1975

Distribution: England, Scotland, Wales, Ireland

Notes: some distribution data from Shaw and Askew (1976)

\section{Colastes (Colastes) fragilis (Haliday, 1836)}

Nomenclature:

Rogas fragilis Haliday, 1836

semeyticus Jakimavičius, 1969 
Distribution: England, Scotland

Colastes (Colastes) incertus (Wesmael, 1838)

Nomenclature:

Exothecus incertus Wesmael, 1838

Distribution: England, Scotland

Colastes (Colastes) magdalenae Sterzynski, 1983

Distribution: England

Notes: NMS, det. Shaw \& van Achterberg, added on Fauna Europaea

Colastes (Colastes) pubicornis (Thomson, 1892)

Nomenclature:

Exothecus pubicornis Thomson, 1892

Distribution: England, Scotland

Notes: added by Godfray and McGavin (1985)

Colastes (Colastes) vividus Papp, 1975

Distribution: England

Notes: NMS, det. Shaw, added here

Subgenus Fungivenator van Achterberg \& Shaw, 2008

Colastes (Fungivenator) sandei van Achterberg \& Shaw, 2008

Distribution: England

Notes: added by van Achterberg and Shaw (2008)

Genus Shawiana van Achterberg, 1983

Nomenclature:

PHANOMERIS misident. 


\section{Shawiana catenator (Haliday, 1836)}

Nomenclature:

Rogas catenator Haliday, 1836

Distribution: England, Scotland, Wales

Notes: some distribution data from Shaw and Askew (1976)

\section{Shawiana laevis (Thomson, 1892)}

Nomenclature:

Exothecus laevis Thomson, 1892

rugulosus (Hellén, 1959, Colastes)

Distribution: England

Notes: NMS, det. Shaw, added on Fauna Europaea

\section{Genus Xenarcha Förster, 1863}

Nomenclature:

ZAMEGASPILUS Ashmead, 1900

Xenarcha abnormis (Wesmael, 1838)

Nomenclature:

Exothecus abnormis Wesmael, 1838

glabricollis (Thomson, 1892, Exothecus)

Distribution: England, Scotland, Wales

Notes: NMS, det. Shaw, added on Fauna Europaea

\section{Xenarcha lustrator (Haliday, 1836)}

Nomenclature:

Rogas lustrator Haliday, 1836

dimidiatus (Nees, 1834, Bracon) preocc.

Iustratrix Schulz, 1906

thomsoni (Szépligeti, 1906, Phanomeris)

Distribution: England, Wales, Ireland 


\section{Gnamptodontinae}

Subfamily Gnamptodontinae Fischer, 1970

Nomenclature:

GNAPTODONTINAE misspelling

GNAPTOGASTRINAE Tobias, 1976

\section{Genus Gnamptodon Haliday, 1836}

Nomenclature:

GNAPTODON Haliday, 1837 suppressed

DIRAPHUS Wesmael, 1838

MESOTAGES Förster, 1863

\section{Gnamptodon decoris (Förster, 1863)}

Nomenclature:

Mesotages decoris Förster, 1863

klemensiewiczii Niezabitowski, 1910

bachmaieri Fischer, 1957

Distribution: England, Wales

Notes: NMS, det. Shaw, added on Fauna Europaea

\section{Gnamptodon pumilio (Nees, 1834)}

Nomenclature:

Bracon pumilio Nees, 1834

pygmaeus (Wesmael, 1838, Diraphus)

Distribution: England, Scotland, Ireland

Notes: some distribution data from Shaw and Askew (1976) 


\section{Helconinae}

Subfamily Helconinae Förster, 1863

Notes: The Diospilini were removed to the Brachistinae by Sharanowski et al. (2011).

\section{Tribe Helconini Förster, 1863}

Notes: Much distribution and taxonomic data from Achterberg (1987).

\section{Genus Helcon Nees, 1814}

Nomenclature:

GYMNOSCELUS Förster, 1863

EDYIA Cameron, 1905

COELOSTEPHANUS Kieffer, 1911

Helcon claviventris Wesmael, 1835

Distribution: England

Notes: added by Achterberg (1987)

Helcon tardator Nees, 1812

Nomenclature:

?adulterator (Villers, 1789, Ichneumon)

Distribution: England

Genus Helconidea Viereck, 1914

Helconidea dentator (Fabricius, 1804)

Nomenclature:

Pimpla dentator Fabricius, 1804

aequator (Nees, 1812, Helcon)

tentator (Thunberg, 1824, Ichneumon) preocc.

rugator (Ratzeburg, 1848, Helcon)

armator (Marshall, 1898, Helcon)

dentatrix (Schulz, 1906, Pimpla) 
Distribution: England

Notes: Helconidea armator (Marshall, 1898, Helcon) removed from synonymy by Achterberg (2014).

Helconidea ruspator (Linnaeus, 1758)

Nomenclature:

Ichneumon ruspator Linnaeus, 1758

dentator (Nees, 1812, Helcon) preocc.

Distribution: England

Genus Wroughtonia Cameron, 1899

Nomenclature:

DUPORTIA Kieffer, 1921

Wroughtonia spinator (Lepeletier, 1825)

Nomenclature:

Helcon spinator Lepeletier, 1825

annulicornis (Nees, 1834, Helcon)

Distribution: England

\section{Homolobinae}

Subfamily Homolobinae van Achterberg, 1979

Genus Homolobus Förster, 1863

Nomenclature:

ZELE misident.

Notes: Shaw (2010) summarises the taxonomy and biology of British and Irish species, including some distribution data. 
Subgenus Apatia Enderlein, 1920

Homolobus (Apatia) truncator (Say, 1828)

Nomenclature:

Bracon truncator Say, 1828

calcarator (Wesmael, 1835, Phylax)

melleus (Cresson, 1872, Phylax)

crassicalcaratus (Viereck, 1905, Zele)

calcaratrix (Schulz, 1906, Zele)

fuscitarsis (Bengtsson, 1918, Phylacter)

simillimus (Enderlein, 1920, Apatia)

unicolor (Enderlein, 1920, Zele)

chlorophthalmus (Nixon, 1938, Zele)

Distribution: England

Notes: Nixon's description of Zele chlorophthalmus validated the use of the name for

this taxon; chlorophthalmus of authors is a misidentification (Bracon chlorophthalmus

Spinola, 1808 is actually a species of the true Zele).

\section{Subgenus Chartolobus van Achterberg, 1979}

Homolobus (Chartolobus) infumator (Lyle, 1914)

Nomenclature:

Zele infumator Lyle, 1914

wesmaeli (Bengtsson, 1918, Phylacter)

japonicus (Watanabe, 1932, Zele)

Distribution: England, Scotland, Wales, Ireland

Subgenus Homolobus Förster, 1863

Homolobus (Homolobus) discolor (Wesmael, 1835)

Nomenclature:

Phylax discolor Wesmael, 1835

pectoralis (Herrich-Schäffer, 1838, Rogas); synonymy by Achterberg (1992b)

Distribution: England, Wales, Ireland 
Subgenus Oulophus van Achterberg, 1979

Homolobus (Oulophus) flagitator (Curtis, 1837)

Nomenclature:

Zele flagitator Curtis, 1837

geminator (Lyle, 1914, Zele)

Distribution: England, Scotland, Wales, Ireland

Subgenus Phylacter Reinhard, 1863

Homolobus (Phylacter) annulicornis (Nees, 1834)

Nomenclature:

Rogas annulicornis Nees, 1834

testaceator misident.

simplex (Herrich-Schäffer, 1838, Rogas); synonymy by Achterberg (1992b)

Distribution: England, Scotland, Ireland

\section{Hormiinae}

Subfamily Hormiinae Förster, 1863

Genus Hormius Nees, 1818

Nomenclature:

CHLIDONIA Herrich-Schäffer, 1838

HORMIELLUS Enderlein, 1912

MEDIELLA Hedqvist, 1963

ANHORMIUS Belokobylskij, 1989

Hormius maderae Graham, 1986

Distribution: England

Notes: NMS, det. Shaw \& van Achterberg, added on Fauna Europaea 
Hormius moniliatus (Nees, 1811)

Nomenclature:

Bracon moniliatus Nees, 1811

brevipennis Hellén, 1957

dusmeti (Docavo Alberti, 1960, Hormiopterus)

insularis Hedqvist, 1965

coniceps Hellén, 1957

Distribution: England, Ireland, Isle of Man

\section{Hormius piciventris Wesmael, 1838}

Distribution: England, Scotland, Wales, Ireland, Isle of Man

Notes: NMS, det. Shaw \& van Achterberg, added on Fauna Europaea. Considered here to be a valid species; van Achterberg (in Belokobylskij et al. 2003) considered that piciventris may be a distinct species but it is listed as a synonym of moniliatus in Taxapad (Yu et al. 2012).

\section{Ichneutinae}

Subfamily Ichneutinae Förster, 1863

Tribe Ichneutini Förster, 1863

Genus Ichneutes Nees, 1814

Ichneutes brevis Wesmael, 1835

Distribution: England, Ireland

Ichneutes reunitor Nees, 1816

Nomenclature:

costatus (Zetterstedt, 1838, Microgaster)

laeviventris Hellén, 1958

leptostigma Hellén, 1958

Distribution: England, Ireland 
Tribe Proteropini van Achterberg, 1976

Genus Proterops Wesmael, 1835

Nomenclature:

ICHNEUTIDEA Ashmead, 1900

PROTEROPOIDES Viereck, 1909

Proterops nigripennis Wesmael, 1835

Distribution: England, Scotland, Ireland

\section{Macrocentrinae}

Subfamily Macrocentrinae Förster, 1863

Genus Austrozele Roman, 1910

Nomenclature:

PANISCOZELE Enderlein, 1920

PALINZELE Brues, 1922

LAEVIS Sharma, 1982

Austrozele longipalpis van Achterberg, 1993

Distribution: England

Notes: added by Achterberg (1993a)

Genus Macrocentrus Curtis, 1833

Nomenclature:

AMICROPLUS Förster, 1863

AMICROPLITES Dalla Torre, 1898

FHOGRA Cameron, 1901

LEPTOZELE Cameron, 1910

METAPLEURODON Enderlein, 1920

PSEUDOPHYLACTER Fahringer, 1929

Notes: Distribution data from Eady and Clark (1964) and Achterberg and Haeselbarth (1983). 


\section{Macrocentrus bicolor Curtis, 1833}

Nomenclature:

limbator (Ratzeburg, 1848, Rogas)

gracilipes Telenga, 1935

Distribution: England, Scotland, Wales, Ireland, Isle of Man

Notes: Achterberg and Haeselbarth (1983) and Achterberg (1993a) treat gracilipes as

a synonym of bicolor but Belokobylskij et al. (2003) list it as a synonym of thoracicus.

\section{Macrocentrus blandus Eady and Clark, 1964}

Distribution: England, Scotland, Ireland

\section{Macrocentrus cingulum Brischke, 1882}

Nomenclature:

grandii Goidanich, 1937

gifuensis misident.

Distribution: England

Macrocentrus collaris (Spinola, 1808)

Nomenclature:

Bracon collaris Spinola, 1808

ebeninus (Nees, 1834, Bracon)

dubius (Wesmael, 1835, Eubadizon)

picipes (Haliday, 1835, Helcon)

dispar (Kollar, 1852, Bracon) preocc.

kollari (Rondani, 1877, Bracon); synonymy by Papp (1996b)

affinis Hedwig, 1961

affiniqades Shenefelt, 1969

Distribution: England, Ireland

Macrocentrus equalis Lyle, 1914

Distribution: England 
Macrocentrus infirmus (Nees, 1834)

Nomenclature:

Rogas infirmus Nees, 1834

Distribution: England, Scotland, Ireland, Isle of Man

\section{Macrocentrus linearis (Nees, 1812)}

Nomenclature:

Bracon linearis Nees, 1812

abdominalis (Fabricius, 1793, Ichneumon) preocc.

abdominator (Thunberg, 1824, Ichneumon)

fissura (Thunberg, 1824, Ichneumon)

pallidator (Zetterstedt, 1838, Bracon) preocc.

tenuis (Ratzeburg, 1848, Rogas)

iridescens French, 1880

gifuensis Ashmead, 1906

amicroploides Viereck, 1912

pallidatorius (Fahringer, 1928, Bracon)

Distribution: England, Scotland, Ireland, Isle of Man

\section{Macrocentrus marginator (Nees, 1811)}

Nomenclature:

Bracon marginator Nees, 1811

rugator (Ratzeburg, 1848, Rogas)

Distribution: England, Scotland, Ireland

Notes: The North American Macrocentrus aegeriae Rohwer, 1915 was removed from synonymy by Achterberg and Haeselbarth (1983).

\section{Macrocentrus nidulator (Nees, 1834)}

Nomenclature:

Rogas nidulator Nees, 1834

longicaudis (Herrich-Schäffer, 1838, Rogas)

procerus Costa, 1884

curticaudis Telenga, 1950 
Distribution: England, Scotland, Wales, Ireland

Macrocentrus nitidus (Wesmael, 1835)

Nomenclature:

Rogas nitidus Wesmael, 1835

Distribution: England, Scotland

Macrocentrus pallipes (Nees, 1811)

Nomenclature:

Bracon pallipes Nees, 1811

pallidipes Dalla Torre, 1898

Distribution: England

Macrocentrus resinellae (Linnaeus, 1758)

Nomenclature:

Ichneumon resinellae Linnaeus, 1758

resinator (Thunberg, 1824, Ichneumon) unavailable

flavipes (Ratzeburg, 1844, Rogas)

interstitialis (Ratzeburg, 1844, Rogas)

obscurator (Ratzeburg, 1848, Rogas)

intricator (Ratzeburg, 1852, Helcon)

punctifrons Thomson, 1895

sublaevis Thomson, 1895

Distribution: England, Scotland

Macrocentrus sylvestrellae van Achterberg, 2001

Distribution: England

Notes: NMS, det. Shaw, added here

Macrocentrus thoracicus (Nees, 1811)

Nomenclature:

Bracon thoracicus Nees, 1811

longicornis (Wesmael, 1835, Rogas) 
Distribution: England, Wales

\section{Macrocentrus townesi van Achterberg \& Haeselbarth, 1983}

Distribution: England, Scotland, Ireland

Notes: added by Achterberg and Haeselbarth (1983)

\section{Meteorinae}

\section{Subfamily Meteorinae Cresson, 1887}

Nomenclature:

ZELINAE Ashmead, 1900

ZEMIOTINAE van Achterberg, 1976

Notes: Stigenberg et al. (2015) included the meteorines as a basal tribe of Euphorinae, rather than as a separate subfamily, as was the preference of many authors, for example, Shaw and Huddleston (1991). In light of their very different developmental biology (parasitoids of larval Lepidoptera and Coleoptera as opposed to parasitising adult insects) and sister-group position to the euphorines, we retain the subfamily rank for Meteorinae.

\section{Genus Meteorus Haliday, 1835}

\section{Nomenclature:}

SAPROTICHUS Holmgren, 1868

PACHYTHECUS Cameron, 1912 preocc.

Notes: Distribution and synonymic data from Huddleston (1980), Stigenberg and Ronquist (2011) and Stigenberg \& Shaw (2013), except where noted. Taxonomy follows Stigenberg and Ronquist (2011).

Species of Meteorus excluded from the British and Irish list:

[flaviceps (Ratzeburg, 1844, Perilitus) nom. dub.]

\section{Meteorus abdominator (Nees, 1811)}

Nomenclature:

Perilitus abdominator Nees, 1811

brunnipes (Ruthe, 1862, Perilitus)

bruneipes Dalla Torre, 1898 
brevipesalis Shenefelt, 1969

?delator (Haliday, 1835, Perilitus); synonymy by Achterberg (1997)

Distribution: England, Scotland, Wales, Ireland

Meteorus abscissus Thomson, 1895

Distribution: England, Scotland, Ireland

Meteorus affinis (Wesmael, 1835)

Nomenclature:

Perilitus affinis Wesmael, 1835

gracilis Ruthe, 1862 preocc.

punctiventris Ruthe, 1862

ruthei Schmiedeknecht, 1897

voloscensis Fischer, 1959

Distribution: England, Scotland, Wales, Ireland

Meteorus alborossicus Lobodenko, 2000

Distribution: England

Notes: added by Stigenberg and Ronquist (2011)

\section{Meteorus brevicauda Thomson, 1895}

Nomenclature:

thuringiacus Schmiedeknecht, 1897

mongolicus Fahringer, 1935

Distribution: England

Notes: Listed by Huddleston (1978) but Huddleston (1980) could not find any British or

Irish material. Shaw (1988b) subsequently recorded it as a British species.

\section{Meteorus cespitator (Thunberg, 1824)}

Nomenclature:

Ichneumon cespitator Thunberg, 1824

atrator (Curtis, 1832, Zele)

similator (Nees, 1834, Perilitus) 
microcerus (Wesmael, 1835, Perilitus)

humeralis (Zetterstedt, 1838, Bracon)

rufipes (Zetterstedt, 1838, Bracon)

ambiguus Ruthe, 1862

Distribution: England, Scotland, Wales, Ireland

\section{Meteorus cinctellus (Spinola, 1808)}

\section{Nomenclature:}

Bracon cinctellus Spinola, 1808

fuscipes (Wesmael, 1835, Perilitus)

Distribution: England, Scotland, Wales

Notes: This has recently been identified as Meteorus necator (e.g. Belokobylskij et al. 2003) but, according to Stigenberg and Ronquist (2011), Ichneumon necator Fabricius, 1777 , is actually a species of Microgastrinae.

\section{Meteorus cis (Bouché, 1834)}

\section{Nomenclature:}

Bracon cis Bouché, 1834

profligator (Haliday, 1835, Perilitus); synonymy by van Achterberg in Belokobylskij et al. (2003)

Distribution: England, Scotland, Wales, Ireland

\section{Meteorus colon (Haliday, 1835)}

Nomenclature:

Perilitus colon Haliday, 1835

fragilis (Wesmael, 1835, Perilitus)

fasciatus (Ratzeburg, 1844, Perilitus)

alternatus Ruthe, 1862

continuus Ruthe, 1862

luridus Ruthe, 1862

pallidus Ruthe, 1862

trivittatus Ruthe, 1862

Distribution: England, Scotland, Wales, Ireland 
Meteorus consimilis (Nees, 1834)

Nomenclature:

Perilitus consimilis Nees, 1834

brevipes (Wesmael, 1835, Perilitus)

albicornis Ruthe, 1862

flagellatus Alexeev, 1971

Distribution: England, Ireland

Meteorus eadyi Huddleston, 1980

Distribution: England, Wales

Notes: added by Huddleston (1980)

Meteorus eklundi Stigenberg, 2011

Distribution: England

Notes: added by Stigenberg and Ronquist (2011)

Meteorus filator (Haliday, 1835)

Nomenclature:

Perilitus filator Haliday, 1835

laticeps (Wesmael, 1835, Perilitus)

hodisensis Fischer, 1970

Distribution: England, Scotland, Ireland

Meteorus heliophilus Fischer, 1970

Distribution: England

Notes: added by Huddleston (1980)

Meteorus hirsutipes Huddleston, 1980

Distribution: England, Ireland

Notes: added by Huddleston (1980) 
Meteorus ictericus (Nees, 1811)

\section{Nomenclature:}

Bracon ictericus Nees, 1811

minutor (Thunberg, 1824, Ichneumon)

lucidator (Trentepohl, 1829, Bracon)

ephippium (Curtis, 1832, Zele)

xanthomelas (Wesmael, 1835, Perilitus)

rubriceps (Ratzeburg, 1844, Perilitus)

confinis Ruthe, 1862

consors Ruthe, 1862

fallax Ruthe, 1862

liquis Ruthe, 1862

pleuralis Ruthe, 1862

crassicrus Thomson, 1895

lophyriphagus Fahringer, 1934

dumbletoni Muesebeck, 1939

adoxophyesi Minamikawa, 1954

makinoharanus Minamikawa, 1954

Distribution: England, Scotland, Wales, Ireland, Isle of Man

Meteorus jaculator (Haliday, 1835)

Nomenclature:

Perilitus jaculator Haliday, 1835

obscurellus Ruthe, 1862

tenuicornis Thomson, 1895

turcicus Fahringer, 1944

Distribution: England, Scotland, Wales, Ireland

\section{Meteorus limbatus Maeto, 1989}

Distribution: England, Scotland

Notes: added by Stigenberg and Ronquist (2011) 
Meteorus lionotus Thomson, 1895

Nomenclature:

ruficoloratus Fischer, 1957

Distribution: England, Scotland

Notes: added by Huddleston (1980)

Meteorus longipilosus Stigenberg, 2011

Distribution: England, Wales

Notes: added by Stigenberg and Shaw (2013)

Meteorus melanostictus Capron, 1887

Nomenclature:

niger Lyle, 1913

monachae Tobias, 1986; synonymy by Belokobylskij (2000b)

Distribution: England

\section{Meteorus micropterus (Haliday, 1835)}

Nomenclature:

Perilitus micropterus Haliday, 1835

Distribution: England, Scotland, Wales, Ireland

Meteorus obfuscatus (Nees, 1811)

Nomenclature:

Bracon obfuscatus Nees, 1811

thoracicus (Curtis, 1832, Zele)

formosus (Wesmael, 1835, Perilitus)

orchesiae (Boie, 1841, Alysia)

fodori Papp, 1973

Distribution: England

Meteorus obsoletus (Wesmael, 1835)

Nomenclature:

Perilitus obsoletus Wesmael, 1835 
viridanae Johansson, 1964

Distribution: England, Scotland, Ireland

Notes: added by Huddleston (1980)

\section{Meteorus oculatus Ruthe, 1862}

Distribution: Scotland

Notes: added by Stigenberg and Shaw (2013)

\section{Meteorus pendulus (Müller, 1776)}

\section{Nomenclature:}

Ichneumon pendulus Müller, 1776

pendulator (Latreille, 1799, Ichneumon)

gyrator (Thunberg, 1824, Ichneumon); synonymy by van Achterberg in Belokobylskij et al. (2003)

ochraceator (Curtis, 1832, Zele) nom. nud.

scutellator (Nees, 1834, Perilitus)

petiolator (Zetterstedt, 1838, Bracon)

parvulus Thomson, 1895

Distribution: England, Scotland, Wales, Ireland

\section{Meteorus pulchricornis (Wesmael, 1835)}

Nomenclature:

Perilitus pulchricornis Wesmael, 1835

striatus Thomson, 1895

thomsoni Marshall, 1899

japonicus Ashmead, 1906

nipponensis Viereck, 1912

baicalensis Telenga, 1950

graeffei Fischer, 1957

macedonicus Fischer, 1957

tuberculifer Fischer, 1957

Distribution: England, Scotland, Wales, Ireland 
Notes: added by Huddleston (1980)

\section{Meteorus rubens (Nees, 1811)}

\section{Nomenclature:}

Bracon rubens Nees, 1811

leviventris (Wesmael, 1835, Perilitus)

islandicus Ruthe, 1859

medianus Ruthe, 1862

vulgaris (Cresson, 1872, Perilitus)

dejanus (Rondani, 1877, Perilitus); synonymy by Papp (1996b)

scutatus Costa, 1884

coquilleti Ashmead, 1889

heteroneurus Thomson, 1895

mellinervis Viereck, 1903

mamestrae Viereck, 1913

szechuanensis Fahringer, 1935

mesopotamicus Fischer, 1957

Distribution: England, Scotland, Wales, Ireland, Isle of Man

\section{Meteorus ruficeps Nees, 1834}

Nomenclature:

pallipes (Wesmael, 1835, Perilitus)

nigritarsis Ruthe, 1862

pallidipes Marshall, 1887

Distribution: England, Scotland, Ireland, Isle of Man

\section{Meteorus rufus (DeGeer, 1773)}

\section{Nomenclature:}

Ichneumon rufus DeGeer, 1773

rufus (Retzius, 1783, Ichneumon) preocc.

unicolor (Wesmael, 1835, Perilitus); synonymy by van Achterberg in Belokobylskij et al. (2003)

chinensis (Holmgren, 1868, Saprotichus) 
Distribution: England, Wales

\section{Meteorus sibyllae Stigenberg, 2011}

Distribution: England, Scotland

Notes: added by Stigenberg and Shaw (2013)

\section{Meteorus sulcatus Szépligeti, 1896}

Nomenclature:

insignis Muesebeck, 1939

molorchi Fischer, 1966

Distribution: England

\section{Meteorus tabidus (Wesmael, 1835)}

\section{Nomenclature:}

Perilitus tabidus Wesmael, 1835

dubius Ruthe, 1862

facialis Ruthe, 1862

pentheri Fischer, 1970

Distribution: England, Scotland, Ireland

\section{Meteorus tenellus Marshall, 1887}

Nomenclature:

boreus Tobias, 1986

Distribution: Scotland

Notes: Added by Stigenberg and Shaw (2013). Separated from cinctellus by Stigenberg and Ronquist (2011); Marshall (1887) gave no locality data so the first documented British occurrence was published by Stigenberg and Shaw (2013).

Meteorus versicolor (Wesmael, 1835)

Nomenclature:

Perilitus versicolor Wesmael, 1835

bimaculatus (Wesmael, 1835, Perilitus)

unicolor (Hartig, 1838, Perilitus) preocc. 
brevicornis (Ratzeburg, 1844, Perilitus) preocc.

rugator (Ratzeburg, 1852, Perilitus)

decoloratus Ruthe, 1862

ikonomovi Fischer, 1959

hartigi Shenefelt, 1969

Distribution: England, Scotland, Wales, Ireland

\section{Meteorus vexator (Haliday, 1835)}

Nomenclature:

Perilitus vexator Haliday, 1835

Distribution: England, Scotland, Ireland

\section{Genus Zele Curtis, 1832}

\section{Nomenclature:}

PROTELUS Förster, 1863

SEMIOTES Förster, 1863

Notes: Synonymic and some distribution data from Achterberg (1979), Achterberg (1984b) and Stigenberg and Shaw (2013). Zele longicauda Curtis, 1832 is apparently a synonym of a species of Eubazus (van Achterberg, pers. comm.).

species of Zele excluded from the British and Irish list:

[annulicrus (Thomson, 1895, Meteorus)] Included in Huddleston's (Huddleston 1978) checklist but we can trace no specimens or literature citations.

\section{Zele albiditarsus Curtis, 1832}

\section{Nomenclature:}

testaceator Curtis, 1832

albitarsis (Nees, 1834, Perilitus)

dispar (Wesmael, 1835, Perilitus)

calcitrator (Curtis, 1837, Meteorus)

wesmaeli (Boie, 1850, Perilitus)

testaceatrix Schulz, 1906

Distribution: England, Scotland, Wales, Ireland 


\section{Zele caligatus (Haliday, 1835)}

Nomenclature:

Meteorus caligatus Haliday, 1835

neesii (Ruthe, 1862, Meteorus)

alaskensis (Ashmead, 1902, Dyscoletes)

sibiricus (Fahringer, 1930, Meteorus)

Distribution: England, Scotland, Wales, Ireland

\section{Zele chlorophthalmus (Spinola, 1808)}

Nomenclature:

Bracon chlorophthalmus Spinola, 1808

chrysophthalmus (Nees, 1811, Bracon)

pallidus (Nees, 1811, Bracon); synonymy by Stigenberg and Ronquist (2011)

nudator (Thunberg, 1824, Ichneumon)

splendens (Costa, 1884, Meteorus)

nigricollis (Thomson, 1895, Meteorus)

Distribution: England, Scotland, Ireland

\section{Zele deceptor (Wesmael, 1835)}

\section{Nomenclature:}

Perilitus deceptor Wesmael, 1835

pallitarsis (Cresson, 1872, Perilitus)

rufulus (Thomson, 1895, Meteorus)

palliditarsis (Dalla Torre, 1898, Meteorus)

maximus (Muesebeck, 1923, Meteorus)

reticulatus (Muesebeck, 1923, Meteorus)

romani (Fahringer, 1930, Meteorus)

separandus (Fischer, 1957, Meteorus)

metallicus (Jakimavičius, 1972, Apanteles)

Distribution: England, Scotland, Wales, Ireland 


\section{Microgastrinae}

\section{Subfamily Microgastrinae Förster, 1863}

Notes: The generic classification of Microgastrinae broadly follows Papp (1988), based on Mason's (Mason 1981) phylogenetic treatment of microgastrines that split up the large genus 'Apanteles', which in turn built upon Nixon's (Nixon 1965) assignment of ' Apanteles' into species groups. We do not follow van Achterberg's (Achterberg 2003c) recent generic revision (reflected in Fauna Europaea), which reassigned many genera to Apanteles and Protapanteles. We consider this reclassification to be premature, being based on very little explicit character evidence and, with its concentration on western Palaearctic species, not really addressing the affinities of most of the world's microgastrine species. However, there are undoubted merits to van Achterberg's treatment as not all of the genera currently employed are well-defined. The following genera are recognised as valid by van Achterberg (2003c): Apanteles (=Choeras, Dolichogenidea, Iconella, Illidops, Pholetesor), Cotesia, Deuterixys, Diolcogaster, Hygroplitis, Microgaster, Microplitis, Paroplitis and Protapanteles (= Distatrix, Glyptapanteles, Rasivalva, Sathon). Some distribution data taken from Nixon (1965), Nixon (1968), Nixon (1970), Nixon (1972), Nixon (1973), Nixon (1974), Nixon (1976), remainder from Shaw (2012) and NMS.

\section{Tribe Apantelini Viereck, 1918}

\section{Genus Apanteles Förster, 1863}

\section{Nomenclature:}

UROGASTER Ashmead, 1898

XESTAPANTELES Cameron, 1910

ALLAPANTELES Brèthes, 1915

AREOLATUS Rao \& Chalikwar, 1976 unavailable

Notes: The generic placement of several species treated by Nixon (1973) in his metacarpalis group, here largely apportioned between Apanteles and Dolichogenidea following Papp (1988), is questionable (cf. Achterberg 2003c).

species of Apanteles excluded from the British and Irish list:

[anomalon (Curtis, 1830, Microgaster)] This name appeared in Huddleston (1978) but is not listed by Papp (1988) or Achterberg (2003c) and remains uninterpreted.

[nigripes (Ratzeburg, 1844, Microgaster)] This name appeared in Huddleston (1978) but is not listed by Papp (1988) or Achterberg (2003a). 
[picipes (Bouché, 1834, Microgaster)] Papp (1987) intended to deal with this name, with a footnote in that paper saying that it would be dealt with under $A$. xanthostigma, but there is no mention there of picipes. It is presumed that the name picipes is a synonym or a nomen dubium; it is not listed as a valid species by Achterberg (2003c).

Apanteles atreus Nixon, 1973

Distribution: England

Apanteles brunnistigma Abdinbekova, 1969

Nomenclature:

sotades Nixon, 1976

Distribution: England, Scotland, Wales, Isle of Man

\section{Apanteles carpatus (Say, 1836)}

Nomenclature:

Microgaster carpata Say, 1836

solitarius (Ashmead,1900)

hawaiiensis (Ashmead, 1901)

fuscicornis (Cameron, 1910)

piceoventris Muesebeck, 1921

igae Watanabe, 1932

sarcitorius Telenga, 1955

ultericus Telenga, 1955

Distribution: England

Apanteles chrysis Nixon, 1973

Distribution: England

\section{Apanteles contaminatus (Haliday, 1834)}

Nomenclature:

Microgaster contaminatus Haliday, 1834

Distribution: Scotland, Ireland 
Apanteles corvinus Reinhard, 1880

Nomenclature:

lucidus Szépligeti, 1896

rasteratus Fahringer, 1936

aptus Papp, 1977

Distribution: England, Scotland

Apanteles galleriae Wilkinson, 1932

Distribution: England

Notes: added by Shaw (2012)

Apanteles lacteus (Nees, 1834)

Nomenclature:

Microgaster lacteus Nees, 1834

Distribution: England

Notes: We follow Mason (1981) in placing lacteus in Apanteles rather than Papp's (Papp 1988) placement in Dolichogenidea.

Apanteles lenea Nixon, 1976

Distribution: England, Scotland, Ireland

Apanteles metacarpalis (Thomson, 1895)

Nomenclature:

Microgaster metacarpalis Thomson, 1895

Distribution: England, Ireland

Apanteles miramis Nixon, 1976

Distribution: England

Apanteles obscurus (Nees, 1834)

Nomenclature:

Microgaster obscura Nees, 1834

arenarius (Haliday, 1834) 
Distribution: England, Ireland

Apanteles sodalis (Haliday, 1834)

Nomenclature:

Microgaster sodalis Haliday, 1834

carbonarius (Ratzeburg, 1848) preocc.

ater (Ratzeburg, 1852); synonymy by Achterberg (1997)

lugens (Ratzeburg, 1852)

lindbergi Hedqvist, 1965

Distribution: England

Apanteles xanthostigma (Haliday, 1834)

Nomenclature:

Microgaster xanthostigma Haliday, 1834

ochrostigma (Wesmael, 1837)

xanthocarpus Szépligeti, 1901

Distribution: England, Scotland, Wales, Isle of Man

Genus Choeras Mason, 1981

Choeras arene (Nixon, 1973)

Nomenclature:

Apanteles arene Nixon, 1973

Distribution: England, Scotland, Ireland

\section{Choeras dorsalis (Spinola, 1808)}

Nomenclature:

Microgaster dorsalis Spinola, 1808

cruciatus (Ratzeburg, 1844)

suffolciensis (Morley, 1902)

Distribution: England, Wales 
Choeras parasitellae (Bouché, 1834)

Nomenclature:

Microgaster parasitellae Bouché, 1834

adjuncta misident.

flavilabris (Ratzeburg, 1844)

rufilabris (Ratzeburg, 1844)

lictorius (Reinhard, 1880)

polypori (Gautier \& Bonnamour, 1930)

Distribution: England, Scotland

Notes: Listed under Apanteles and Microgaster in Huddleston (1978).

Choeras ruficornis (Nees, 1834)

Nomenclature:

Microgaster ruficornis Nees, 1834

hedymeles (Nixon, 1973)

Distribution: England

Choeras tedellae (Nixon, 1961)

Nomenclature:

Apanteles tedellae Nixon, 1961

epinotiae (Fischer, 1962)

epinoticida (Fischer, 1966)

Distribution: England

Choeras tiro (Reinhard, 1880)

Nomenclature:

Microgaster tiro Reinhard, 1880

Distribution: England

Choeras validus (Thomson, 1895)

Nomenclature:

Apanteles validus Thomson, 1895

Distribution: England 


\section{Genus Dolichogenidea Viereck, 1911}

Notes: species of Dolichogenidea excluded from the British and Irish list:

[anarsiae (Faure \& Alabouvette, 1924, Apanteles)] Listed as a British species by Huddleston (1978) in error, there are no British records.

[cerialis (Nixon, 1976, Apanteles); syn. areolaris (Balevski \& Tobias, 1980, Apanteles) preocc.] Listed as a British species by Huddleston (1978) in error, only known from southern and Eastern Europe and Israel.

[ensiformis (Ratzeburg, 1844, Microgaster)] Listed as a British species by Huddleston (1978) in error (see note under Napamus vipio).

[evonymellae (Bouché, 1834, Microgaster); syn. iarbas (Nixon, 1972, Apanteles)] Listed as a British species by Huddleston (1978) in error, there are no British records.

[impura (Nees, 1834, Microgaster)] Notwithstanding Papp (1978), we regard this name as uncertainly interpreted, but in any case we have not seen British material that conforms to Papp's interpretation.

\section{Dolichogenidea agilla (Nixon, 1972)}

Nomenclature:

Apanteles agilla Nixon, 1972

piratica (Papp, 1977)

Distribution: England

Notes: added by Shaw (2012)

Dolichogenidea annularis (Haliday, 1834)

Nomenclature:

Microgaster annularis Haliday, 1834

Distribution: England

Dolichogenidea appellator (Telenga, 1949)

Nomenclature:

Apanteles appellator Telenga, 1949

litae (Nixon, 1972)

Distribution: England 
Notes: Added by Shaw (2012). Papp (1988) notes, but does not follow, the view that appellator may be the valid name (cf. Kotenko and Tobias 1986). Shaw (2012) has found that reared 'appellator' and 'litae' appear to be conspecific. The situation is complicated by Nixon's (Nixon 1972) treatment of some series (from a different host in Cyprus, and from Egypt) as 'litae var operculellae', and it is this that Papp (1988)lists as a junior synonym of appellator.

Dolichogenidea artissima (Papp, 1971)

Nomenclature:

Apanteles artissimus Papp, 1971

abila (Nixon, 1972)

Distribution: England, Scotland, Wales

Dolichogenidea ate (Nixon, 1973)

Nomenclature:

Apanteles ate Nixon, 1973

Distribution: England

Dolichogenidea bres (Nixon, 1973)

Nomenclature:

Apanteles bres Nixon, 1973

Distribution: England

Dolichogenidea breviventris (Ratzeburg, 1848)

Nomenclature:

Microgaster breviventris Ratzeburg, 1848

mesoxantha (Ruschka, 1917)

nilae (Telenga, 1961)

Distribution: England, Scotland, Wales, Ireland

Dolichogenidea britannica (Wilkinson, 1941)

Nomenclature:

Apanteles britannicus Wilkinson, 1941

Distribution: England 
Dolichogenidea candidata (Haliday, 1834)

Nomenclature:

Microgaster candidatus Haliday, 1834

longicauda (Wesmael, 1837); synonymy by Achterberg (1997)

terebrator (Ratzeburg, 1852)

Distribution: England, Scotland, Wales, Isle of Man

Dolichogenidea coleophorae (Wilkinson, 1938)

Nomenclature:

Apanteles coleophorae Wilkinson, 1938

Distribution: England

Dolichogenidea coniferae (Haliday, 1834)

Nomenclature:

Microgaster coniferae Haliday, 1834

Distribution: England, Isle of Man

Notes: Revised status (Shaw 2012): inadvertently listed as a synonym of candidata by

Achterberg (1997) due to a drafting error (van Achterberg, pers. comm.).

Dolichogenidea credne (Nixon, 1973)

Nomenclature:

Apanteles credne Nixon, 1973

Distribution: England

Dolichogenidea cytherea (Nixon, 1972)

Nomenclature:

Apanteles cytherea Nixon, 1972

Distribution: England

Dolichogenidea decora (Haliday, 1834)

Nomenclature:

Microgaster decorus Haliday, 1834

lineata (Reinhard, 1880) 
sibirica Fahringer, 1938

Distribution: Ireland

Dolichogenidea dilecta (Haliday, 1834)

Nomenclature:

Microgaster dilectus Haliday, 1834

femoralis (Bouché, 1834)

Distribution: England, Isle of Man

Dolichogenidea drusilla (Nixon, 1972)

Nomenclature:

Apanteles drusilla Nixon, 1972

Distribution: England

Dolichogenidea emarginata (Nees, 1834)

Nomenclature:

Microgaster emarginatus Nees, 1834

scapularis (Bouché,1834)

Distribution: England, Scotland, Wales

Dolichogenidea exilis (Haliday, 1834)

Nomenclature:

Microgaster exilis Haliday, 1834

Distribution: England

Notes: Shaw (2012) gives a diagnosis; not treated by Nixon or Papp.

Dolichogenidea faucula (Nixon, 1972)

Nomenclature:

Apanteles faucula Nixon, 1972

Distribution: England 
Dolichogenidea gagates (Nees, 1834)

Nomenclature:

Microgaster gagates Nees, 1834

Distribution: England

Dolichogenidea glabra (Papp, 1978)

Nomenclature:

Apanteles glaber Papp, 1978

Distribution: England, Scotland

Notes: added by Shaw (2012)

Dolichogenidea gracilariae (Wilkinson, 1940)

Nomenclature:

Apanteles gracilariae Wilkinson, 1940

Distribution: England, Isle of Man

Dolichogenidea halidayi (Marshall, 1872)

Nomenclature:

Apanteles halidayi Marshall, 1872

albipennis (Haliday, 1834) preocc.

halidaii misspelling

Distribution: England, Scotland, Isle of Man

Dolichogenidea hilaris (Haliday, 1834)

Nomenclature:

Microgaster hilaris Haliday, 1834

Distribution: Ireland

Dolichogenidea imperator (Wilkinson, 1939)

Nomenclature:

Apanteles imperator Wilkinson, 1939

Distribution: England, Scotland, Wales, Isle of Man 
Dolichogenidea infima (Haliday, 1834)

Nomenclature:

Microgaster infimus Haliday, 1834

Distribution: England

Dolichogenidea lacteicolor (Viereck, 1911)

Nomenclature:

Apanteles lacteicolor Viereck, 1911

conspersae (Fiske, 1911)

Distribution: England

Dolichogenidea lacteipennis (Curtis, 1830)

Nomenclature:

Microgaster lacteipennis Curtis, 1830

lissonota (Tobias, 1964)

Dolichogenidea laevigata (Ratzeburg, 1848)

Nomenclature:

Microgaster laevigatus Ratzeburg, 1848

hoplites (Ratzeburg, 1848)

calcarata (Ivanov, 1899)

Distribution: England, Scotland, Wales

Dolichogenidea laevigatoides (Nixon, 1972)

Nomenclature:

Apanteles laevigatoides Nixon, 1972

Distribution: England

Dolichogenidea laevissima (Ratzeburg, 1848)

Nomenclature:

Microgaster laevissimus Ratzeburg, 1848

tersa (Papp, 1973)

Distribution: England 
Dolichogenidea lemariei (Nixon, 1961)

Nomenclature:

Apanteles lemariei Nixon, 1961

Distribution: England

Dolichogenidea lineipes (Wesmael, 1837)

Nomenclature:

Microgaster lineipes Wesmael, 1837

Distribution: England, Scotland, Isle of Man

Dolichogenidea longicalcar (Thomson, 1895)

Nomenclature:

Apanteles longicalcar Thomson, 1895

Distribution: England

Dolichogenidea longipalpis (Reinhard, 1880)

Nomenclature:

Apanteles longipalpis Reinhard, 1880

tadzhica (Telenga, 1949)

Distribution: England, Scotland

Notes: Papp (1981) reports that the type series of tadzhica belongs to two species ( lacteus and longipalpis) but did not select a lectotype; Belokobylskij et al. (2003) treated the name as a synonym of longipalpis.

Dolichogenidea marica (Nixon, 1972)

Nomenclature:

Apanteles marica Nixon, 1972

Distribution: England

Dolichogenidea myron (Nixon, 1973)

Nomenclature:

Apanteles myron Nixon, 1973

Distribution: England, Scotland 
Notes: Transferred from Apanteles in anticipation of publication by Jose FernandezTriana.

Dolichogenidea ononidis (Marshall, 1889)

Nomenclature:

Apanteles ononidis Marshall, 1889

Distribution: England

Notes: Shaw (2012) gives a diagnosis; not treated by Nixon or Papp.

Dolichogenidea petrovae (Walley, 1937)

Nomenclature:

Apanteles petrovae Walley, 1937

dioryctriae (Wilkinson, 1938)

magna (Telenga, 1955)

murinanae (Čapek \& Zwölfer, 1957)

Distribution: England

Notes: added by Shaw (2012)

Dolichogenidea phaloniae (Wilkinson, 1940)

Nomenclature:

Apanteles phaloniae Wilkinson, 1940

Distribution: England, Scotland, Wales, Ireland

Dolichogenidea phaola (Nixon, 1972)

Nomenclature:

Apanteles phaola Nixon, 1972

Distribution: England

Dolichogenidea praetor (Marshall, 1885)

Nomenclature:

Apanteles praetor Marshall, 1885

Distribution: England 
Dolichogenidea princeps (Wilkinson, 1941)

Nomenclature:

Apanteles princeps Wilkinson, 1941

Distribution: England, Scotland, Wales

Dolichogenidea punctiger (Wesmael, 1837)

Nomenclature:

Microgaster punctiger Wesmael, 1837

itea (Nixon, 1972)

Distribution: England, Scotland

Dolichogenidea sicaria (Marshall, 1885)

Nomenclature:

Apanteles sicarius Marshall, 1885

chrysosticta (Marshall, 1899)

crudelis (Papp, 1971)

Distribution: England, Scotland

Dolichogenidea sisenna (Nixon, 1972)

Nomenclature:

Apanteles sisenna Nixon, 1972

Distribution: England

Notes: Recorded as British by Nixon (1972) but omitted by Huddleston (1978).

Dolichogenidea soikai (Nixon, 1972)

Nomenclature:

Apanteles soikai Nixon, 1972

Distribution: England

Notes: added by Shaw (2012)

Dolichogenidea trachala (Nixon, 1965)

Nomenclature:

Apanteles trachalus Nixon, 1965 
sevocata (Papp, 1975)

Distribution: England, Scotland, Wales, Ireland

Notes: \#A parasitoid of synanthropic Lepidoptera species and probably introduced to Britain and Ireland (Nixon 1976).

\section{Dolichogenidea ultor (Reinhard, 1880)}

Nomenclature:

Apanteles ultor Reinhard, 1880

lactipennis (Ratzeburg, 1852) preocc.

Distribution: England

\section{Dolichogenidea victor (Wilkinson, 1941)}

Nomenclature:

Apanteles victor Wilkinson, 1941

Distribution: England

Genus Illidops Mason, 1981

Illidops butalidis (Marshall, 1888)

Nomenclature:

Apanteles butalidis Marshall, 1888

Distribution: England, Scotland

Illidops naso (Marshall, 1885)

Nomenclature:

Apanteles naso Marshall, 1885

contortus (Tobias, 1964)

crantor (Nixon, 1965)

evander (Nixon, 1965)

coresia (Nixon, 1973)

Distribution: England 
Illidops suevus (Reinhard, 1880)

Nomenclature:

Apanteles suevus Reinhard, 1880

minutus (Szépligeti, 1896)

polonicus (Fahringer, 1936)

brevisternis (Tobias, 1964)

suspicax (Tobias, 1964)

dion (Nixon, 1965)

sesostris (Nixon, 1976)

Distribution: England

\section{Genus Napamus Papp, 1993}

Notes: species of Napamus excluded from the British and Irish list:

[vipio (Reinhard, 1880, Apanteles)] Mistakenly listed as a British species by Kloet and Hincks (1945) and Huddleston (1978). Shenefelt (1973) listed it as British on the misunderstanding that Morley and Rait-Smith (1933) had produced a catalogue of British Lepidoptera-parasitoid asscociations, whereas their listings also included nonBritish rearings from hosts that occur in Britain. Morley and Rait-Smith (1933) cite Marshall (1896) as the source of host records for vipio, who does not mention Britain. Apanteles vipio was transferred from Illidops by Papp (1993).

Genus Pholetesor Mason, 1981

Pholetesor arisba (Nixon, 1973)

Nomenclature:

Apanteles arisba Nixon, 1973

Distribution: England, Scotland

\section{Pholetesor bicolor (Nees, 1834)}

Nomenclature:

Microgaster bicolor Nees, 1834

ardeaepenellae (Bouché, 1834)

pedias (Nixon, 1973)

umbellatarum (Haliday, 1834)

schillei (Niezabitowski, 1910) 
longicauda (Fahringer, 1938)

Distribution: England

Notes: Some distribution data from Shaw and Askew (1976). Van Achterberg's (Achterberg 1997) synonymy of bicolor under circumscriptus is not followed here (Shaw 2012); it is probable that his reared series represents two species, bicolor and circumscriptus.

\section{Pholetesor circumscriptus (Nees, 1834)}

Nomenclature:

Microgaster circumscriptus Nees, 1834

exiguus (Haliday, 1834); synonymy by Achterberg (1997)

blancardellae (Bouché, 1834)

lividipes (Wesmael, 1837)

flavolimbatus (Ratzeburg, 1848)

lautellus (Marshall, 1895)

Distribution: England, Scotland, Wales, Ireland

Pholetesor elpis (Nixon, 1973)

Nomenclature:

Apanteles elpis Nixon, 1973

girkanus (Tobias, 1976)

Distribution: England

Pholetesor errans (Nixon, 1973)

Nomenclature:

Apanteles errans Nixon, 1973

arenicola (Papp, 1973)

Distribution: England

\section{Pholetesor laetus (Marshall, 1885)}

Nomenclature:

Apanteles laetus Marshall, 1885

exiguus misident.

salalicus misident. 
metallicus (Jakimavičius, 1972)

Distribution: England, Scotland

Notes: Shaw (2012) tentatively identified exiguus sensu Nixon (1973) as an extreme of morphological variation within laetus.

\section{Pholetesor maritimus (Wilkinson, 1941)}

Nomenclature:

Apanteles maritimus Wilkinson, 1941

Distribution: England, Scotland, Wales

\section{Pholetesor moldavicus (Tobias, 1975)}

Nomenclature:

Apanteles moldavicus Tobias, 1975

Distribution: England

Notes: Added by Shaw (2012). Although this generic placement (Papp 1988) was followed by Shaw (2012) is seems very likely to be inappropriate.

\section{Pholetesor nanus (Reinhard, 1880)}

Nomenclature:

Apanteles nanus Reinhard, 1880

szoecsi (Papp, 1973)

Distribution: England, Scotland, Wales, Isle of Man

\section{Pholetesor phaetusa (Nixon, 1973)}

Nomenclature:

Apanteles phaetusa Nixon, 1973

Distribution: England, Scotland

\section{Pholetesor viminetorum (Wesmael, 1837)}

Nomenclature:

Microgaster viminetorum Wesmael, 1837

fuliginosus (Wesmael, 1837)

Distribution: England, Scotland, Wales, Ireland 
Tribe Cotesiini Mason, 1981

\section{Genus Cotesia Cameron, 1891}

\section{Nomenclature:}

CRYPTAPANTELES Viereck, 1910

STENOPLEURA Viereck, 1911

Notes: Some taxonomic and distribution data for species parasitizing Lycaenidae taken from Shaw (2007).

species of Cotesia excluded from the British and Irish list:

[acuminata (Reinhard, 1880, Apanteles); cultrator (Marshall, 1885, Apanteles)] Not a British or Irish species. Marshall's description was based on unprovenanced material reared from a host that, on inspection by MRS, is almost certainly not British. The current synonymy is also in doubt.

[ordinaria (Ratzeburg, 1844, Microgaster); syn. dendrolimi (Matsumura, 1926, Apanteles); dendrolimusi (Matsumura, 1926, Apanteles)] Although listed as a British species by various authors we can find no evidence that it has occurred here.

[saltator (Thunberg, 1824, Ichneumon) preocc.] Appeared in Huddleston (1978) as Roman (1912) incorrectly synonymised tenebrosa under saltator.

[scabricula (Reinhard, 1880, Apanteles); syn. eguchii (Watanabe, 1935, Apanteles)] No evidence that this is a British or Irish species.

\section{Cotesia abjecta (Marshall, 1885)}

\section{Nomenclature:}

Apanteles abjectus Marshall, 1885

complanata (Lyle, 1916, Apanteles)

Distribution: England, Scotland

\section{Cotesia affinis (Nees, 1834)}

\section{Nomenclature:}

Microgaster affinis Nees, 1834

euphorbiae (Bouché, 1834, Microgaster)

vinulae (Bouché, 1834, Microgaster)

harpyiae (Niezabitowski, 1910, Apanteles)

okamotoi (Watanabe, 1932, Apanteles) 
planus (Watanabe, 1932, Apanteles)

Distribution: England

Cotesia analis (Nees, 1834)

Nomenclature:

Microgaster analis Nees, 1834

praetextata (Haliday, 1834, Microgaster)

mediana (Ratzeburg, 1852, Microgaster)

leucaniae (Wikinson, 1937, Apanteles)

Distribution: England

\section{Cotesia astrarches (Marshall, 1889)}

\section{Nomenclature:}

Apanteles astrarches Marshall, 1889

?arctica (Thomson, 1895, Apanteles)

genalis (Tobias, 1964, Apanteles)

Distribution: England, Scotland, Wales

Notes: Nixon's (Nixon 1974) arctica encompassed two species that occur in Britain, astrarches and tenebrosa. It is not clear which, if either, the name arctica is associated with. The name astrarches was placed, erroneosly, in synonymy with arctica by Nixon (1974), which was followed by Huddleston (1978). Shaw (2007) clarified the status of astrarches.

\section{Cotesia bignellii (Marshall, 1885)}

Nomenclature:

Apanteles bignellii Marshall, 1885

Distribution: England, Scotland, Wales, Ireland

\section{Cotesia brevicornis (Wesmael, 1837)}

\section{Nomenclature:}

Microgaster brevicornis Wesmael, 1837

cleoceridis (Marshall, 1889, Apanteles)

Distribution: England, Scotland 
Cotesia cajae (Bouché, 1834)

Nomenclature:

Microgaster cajae Bouché, 1834

difficilis (Nees, 1834, Microgaster)

Distribution: England

Cotesia callimone (Nixon, 1974)

Nomenclature:

Apanteles callimone Nixon, 1974

scelerata (Tobias, 1986, Apanteles)

Distribution: Scotland, Wales, Ireland

Cotesia chares (Nixon, 1965)

Nomenclature:

Apanteles chares Nixon, 1965

Distribution: England

Cotesia cleora (Nixon, 1974)

Nomenclature:

Apanteles cleora Nixon, 1974

Distribution: England

Cotesia coryphe (Nixon, 1974)

Nomenclature:

Apanteles coryphe Nixon, 1974

Distribution: England

Notes: Papp (1987) synonymised coryphe under rubripes but this seems unwarranted, given the distinctly different hosts and other aspects of biology.

Cotesia cuprea (Lyle, 1925)

Nomenclature:

Apanteles cupreus Lyle, 1925

Distribution: England, Scotland 
Cotesia errator (Nixon, 1974)

Nomenclature:

Apanteles errator Nixon, 1974

Distribution: England, Wales

Cotesia eulipis (Nixon, 1974)

Nomenclature:

Apanteles eulipis Nixon, 1974

eulipsis misspelling

Distribution: England, Scotland

? Cotesia euryale (Nixon, 1974)

Nomenclature:

Apanteles euryale Nixon, 1974

Distribution: England

Notes: It is not clear from Nixon (1974) that this species occurs in Britain, despite its being 'bred in captivity at Slough'.

Cotesia ferruginea (Marshall, 1885)

Nomenclature:

Apanteles ferrugineus Marshall, 1885

Distribution: England

Cotesia gades (Nixon, 1974)

Nomenclature:

Apanteles gades Nixon, 1974

Distribution: England

Notes: added by Allen (1978)

Cotesia gastropachae (Bouché, 1834)

Nomenclature:

Microgaster gastropachae Bouché, 1834

Distribution: England, Scotland, Isle of Man 


\section{Cotesia geryonis (Marshall, 1885)}

Nomenclature:

Apanteles geryonis Marshall, 1885

Distribution: England

\section{Cotesia glomerata (Linnaeus, 1758)}

\section{Nomenclature:}

$$
\begin{aligned}
& \text { Ichneumon glomeratus Linnaeus, } 1758 \\
& \text { glomerator (Thunberg, 1824, Ichneumon) } \\
& \text { nigriventris (Nees, 1834, Microgaster) } \\
& \text { recondita (Nees, 1834, Microgaster) } \\
& \text { stellatarum (Bouché, 1834, Microgaster) } \\
& \text { crataegi (Ratzeburg, 1844, Microgaster) } \\
& \text { oleracea (Taylor, 1860, Microgaster) } \\
& \text { pieridis (Packard, 1881, Microgaster) preocc. } \\
& \text { pieridivora (Riley, 1882, Microgaster) } \\
& \text { aporiae (Ivanov, 1899, Apanteles) } \\
& \text { nawaii (Ashmead, 1906, Glyptapanteles) } \\
& \text { aporiae (Matsumura, 1908, Apanteles) preocc. } \\
& \text { heterotergis (Fahringer, 1936, Apanteles) }
\end{aligned}
$$

Distribution: England, Scotland, Isle of Man

\section{Cotesia gonopterygis (Marshall, 1885)}

Nomenclature:

Apanteles gonopterygis Marshall, 1885

Distribution: England

\section{Cotesia hyphantriae (Riley, 1887)}

Nomenclature:

Apanteles hyphantriae Riley, 1887

Distribution: England, Wales 
Cotesia inducta (Papp, 1973)

Nomenclature:

Apanteles inductus Papp, 1973

tenuivalvis (Tobias, 1986, Apanteles)

Distribution: England, Ireland

Notes: added by Revels (2006); Shaw (2007)

Cotesia isolde (Nixon, 1974)

Nomenclature:

Apanteles isolde Nixon, 1974

Distribution: England, Scotland

Cotesia jucunda (Marshall, 1885)

Nomenclature:

Apanteles jucundus Marshall, 1885

nigrinervis (Thomson, 1895, Microgaster)

Distribution: England, Scotland

Cotesia juniperatae (Bouché, 1834)

Nomenclature:

Microgaster juniperatae Bouché, 1834

Distribution: England, Scotland

Cotesia kurdjumovi (Telenga, 1955)

Nomenclature:

Apanteles kurdjumovi Telenga, 1955

laverna (Nixon, 1974, Apanteles)

Distribution: England, Scotland

Cotesia limbata (Marshall, 1885)

Nomenclature:

Apanteles limbatus Marshall, 1885

kawadai (Watanabe, 1934, Apanteles) 
Distribution: England, Scotland

Cotesia lineola (Curtis, 1830)

Nomenclature:

Microgaster lineola Curtis, 1830

gabrielis (Gautier \& Riel, 1919, Apanteles)

Distribution: England

Cotesia melanoscela (Ratzeburg, 1844)

Nomenclature:

Microgaster melanoscelus Ratzeburg, 1844

solitaria (Ratzeburg, 1844, Microgaster)

creata (Balevski, 1980, Apanteles)

Distribution: England

Cotesia melitaearum (Wilkinson, 1937)

Nomenclature:

Apanteles melitaearum Wilkinson, 1937

melittaearum misspelling

ukrainica (Tobias, 1986, Apanteles)

Distribution: England, Scotland, Wales

Cotesia notha (Marshall, 1885)

Nomenclature:

Apanteles nothus Marshall, 1885

Distribution: England, Scotland

Cotesia numen (Nixon, 1974)

Nomenclature:

Apanteles numen Nixon, 1974

Distribution: England, Scotland 


\section{Cotesia ofella (Nixon, 1974)}

Nomenclature:

Apanteles ofella Nixon, 1974

?perspicua (Nees, 1834, Microgaster)

Distribution: England

Notes: Microgaster perspicua is listed as a senior synonym of cajae in Taxapad (Yu et al. 2012), following Marshall (1885), who listed cajae as the senior synonym. Papp (2005a) lists perspicua as a tentative synonym (which would have priority) of ofella.

\section{Cotesia onaspis (Nixon, 1974)}

Nomenclature:

Apanteles onaspis Nixon, 1974

avetyanae (Tobias, 1976, Apanteles)

Distribution: England

\section{Cotesia orestes (Nixon, 1974)}

Nomenclature:

Apanteles orestes Nixon, 1974

Distribution: England

\section{Cotesia pilicornis (Thomson, 1890)}

Nomenclature:

Microgaster pilicornis Thomson, 1890

piliflagellaris (Tobias, 1986, Apanteles)

Distribution: England, Scotland, Wales, Ireland

Notes: In NMS numerous series reared solitarily from various species of Pterophoridae are probably conspecific but show a great variation in extent of pilosity of the antenna.

\section{Cotesia praepotens (Haliday, 1834)}

\section{Nomenclature:}

Microgaster praepotens Haliday, 1834

placida (Haliday, 1834, Microgaster)

memnon (Nixon, 1974, Apanteles) 
acutivalvis (Balevski, 1980, Apanteles)

beshtaui (Tobias, 1986, Apanteles)

Distribution: England, Ireland

Notes: Nixon's (Nixon 1974) praepotens is apparently correctly called sericea (Belokobylskij et al. 2003).

\section{Cotesia risilis (Nixon, 1974)}

Nomenclature:

Apanteles risilis Nixon, 1974

Distribution: England

\section{Cotesia rubecula (Marshall, 1885)}

Nomenclature:

Apanteles rubecula Marshall, 1885

Distribution: England

\section{Cotesia rubripes (Haliday, 1834)}

Nomenclature:

Microgaster rubripes Haliday, 1834

Distribution: England, Scotland

\section{Cotesia ruficrus (Haliday, 1834)}

Nomenclature:

Microgaster ruficrus Haliday, 1834

antipoda (Ashmead, 1900, Apanteles)

manilae (Ashmead, 1904, Apanteles)

sydneyensis (Cameron, 1911, Apanteles)

narangae (Viereck, 1913, Apanteles)

sesamiae (Risbec, 1956, Apanteles) nom. nud.

Distribution: England, Wales, Isle of Man 
Cotesia salebrosa (Marshall, 1885)

Nomenclature:

Apanteles salebrosus Marshall, 1885

callunae Nixon, 1974

Distribution: England, Scotland

Cotesia saltatoria (Balevski, 1980)

Nomenclature:

Apanteles saltatorius Balevski, 1980

Distribution: England, Scotland

Notes: added by Shaw (2007)

Cotesia sericea (Nees, 1834)

Nomenclature:

Microgaster sericeus Nees, 1834

praepotens misident.

brachycera (Thomson, 1895, Apanteles)

Distribution: England, Scotland

Notes: Nixon's (Nixon 1974) praepotens is apparently correctly called sericea (Belokobylskij et al. 2003).

Cotesia sibyllarum (Wilkinson, 1936)

Nomenclature:

Apanteles sibyllarum Wilkinson, 1936

Distribution: England

Cotesia spuria (Wesmael, 1837)

Nomenclature:

Microgaster spurius Wesmael, 1837

insidens (Ratzeburg, 1844, Microgaster)

Distribution: England, Scotland, Wales, Isle of Man 
Cotesia subordinaria (Tobias, 1976)

Nomenclature:

Apanteles subordinarius Tobias, 1976

Distribution: England

Notes: added by Shaw (2012)

Cotesia telengai (Tobias, 1972)

Nomenclature:

Apanteles telengai Tobias, 1972

amabilis (Nixon, 1974, Apanteles)

Distribution: England

\section{Cotesia tenebrosa (Wesmael, 1837)}

Nomenclature:

Microgaster tenebrosus Wesmael, 1837

?arctica (Thomson, 1895, Apanteles)

Distribution: England, Scotland

Notes: Added by Shaw (2007); Nixon's (Nixon 1974) arctica encompassed two species that occur in Britain, astrarches and tenebrosa. It is not clear which, if either, the name arctica is associated with. The name astrarches was placed, erroneosly, in synonymy with arctica by Nixon (1974), which was followed by Huddleston (1978). Shaw (2007) clarified the status of tenebrosa.

\section{Cotesia tetrica (Reinhard, 1880)}

Nomenclature:

Apanteles tetricus Reinhard, 1880

opacula (Thomson, 1895, Microgaster)

Distribution: England, Scotland

\section{Cotesia tibialis (Curtis, 1830)}

\section{Nomenclature:}

Microgaster tibialis Curtis, 1830

atrator (Curtis, 1830, Microgaster)

gracilis (Curtis, 1830, Microgaster) 


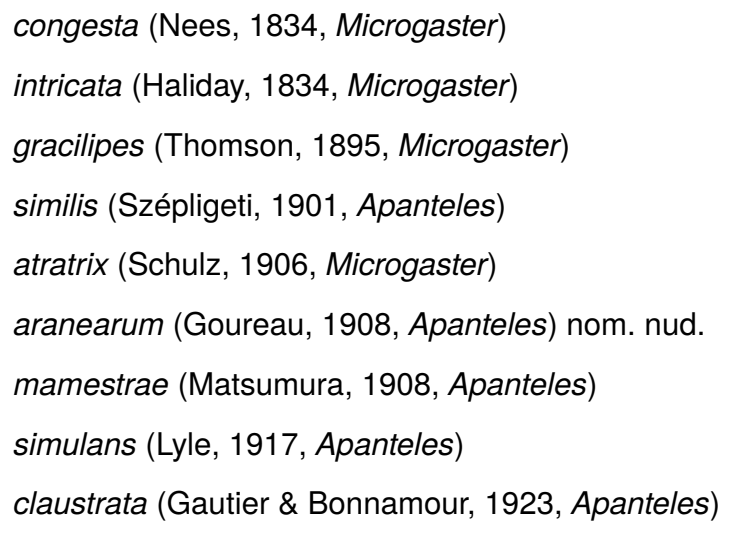

Distribution: England, Scotland, Wales

\section{Cotesia vanessae (Reinhard, 1880)}

Nomenclature:

Apanteles vanessae Reinhard, 1880

Distribution: England

\section{Cotesia vestalis (Haliday, 1834)}

Nomenclature:

Microgaster vestalis Haliday, 1834

plutellae (Kurdjumov, 1912, Apanteles); synonymy by Shaw (2003b)

Distribution: England, Wales

\section{Cotesia villana (Reinhard, 1880)}

Nomenclature:

Apanteles villanus Reinhard, 1880

fasciatae (Gautier \& du Dresnay, 1926, Apanteles)

rubroides (Papp, 1971, Apanteles); synonymy by Papp (2009a)

Distribution: England

\section{Cotesia zygaenarum (Marshall, 1885)}

Nomenclature:

Apanteles zygaenarum Marshall, 1885

Distribution: England, Scotland, Wales, Ireland, Isle of Man 
Genus Deuterixys Mason, 1981

Deuterixys carbonaria (Wesmael, 1837)

Nomenclature:

Microgaster carbonarius Wesmael, 1837

anomala (Lyle, 1925, Apanteles)

Distribution: England, Scotland

Deuterixys plugarui (Tobias, 1975)

Nomenclature:

Apanteles plugarui Tobias, 1975

Distribution: England

Notes: added by Shaw (2012)

Deuterixys rimulosa (Niezabitowski, 1910)

Nomenclature:

Apanteles rimulosus Niezabitowski, 1910

comes (Wilkinson, 1940, Apanteles)

Distribution: England

Genus Diolcogaster Ashmead, 1901

Nomenclature:

PROTOMICROPLITIS misident.

ZADIOLOCOGASTER Viereck, 1913

Notes: See Mason (1981) and Fernández-Triana (2015), who restricted Protomicroplitis to a few Nearctic and Neotropical species.

Diolcogaster abdominalis (Nees, 1834)

Nomenclature:

Microgaster abdominalis Nees, 1834

Distribution: England 
Diolcogaster alvearia (Fabricius, 1798)

Nomenclature:

Ichneumon aleuarius Fabricius, 1798

aphidum (Panzer, 1804, Ichneumon)

alveator (Thunberg, 1824, Ichneumon)

areolata (Szépligeti, 1896, Ichneumon)

Distribution: England, Scotland

Diolcogaster connexa (Nees, 1834)

Nomenclature:

Microgaster connexus Nees, 1834

consularis (Haliday, 1834, Microgaster)

diluta (Ratzeburg, 1852, Microgaster)

Distribution: England, Isle of Man

Diolcogaster flavipes (Haliday, 1834)

Nomenclature:

Microgaster flavipes Haliday, 1834

Diolcogaster hinzi (Nixon, 1965)

Nomenclature:

Protomicroplitis hinzi Nixon, 1965

Distribution: England, Scotland

Notes: added by Shaw (2012)

Diolcogaster minuta (Reinhard, 1880)

Nomenclature:

Microgaster minutus Reinhard, 1880

Distribution: England, Scotland

Diolcogaster scotica (Marshall, 1885)

Nomenclature:

Microgaster scoticus Marshall, 1885 
Distribution: England, Scotland

Diolcogaster spreta (Marshall, 1885)

Nomenclature:

Microgaster spretus Marshall, 1885

Distribution: England, Scotland, Wales

Genus Distatrix Mason, 1981

Distatrix formosa (Wesmael, 1837)

Nomenclature:

Microgaster formosus Wesmael, 1837

marshallii (Bignell, 1901, Apanteles)

Distribution: England, Wales, Isle of Man

Genus Glyptapanteles Ashmead, 1905

Notes: species of Glyptapanteles excluded from the British and Irish list:

[thompsoni (Lyle, 1917, Apanteles)] Listed as a British species by Huddleston (1978) in error; there is no evidence that it has occurred here.

Glyptapanteles acasta (Nixon, 1973)

Nomenclature:

Apanteles acasta Nixon, 1973

Distribution: England

Glyptapanteles aliphera (Nixon, 1973)

Nomenclature:

Apanteles aliphera Nixon, 1973

aliphaera misspelling

sublateralis (Tobias, 1976, Apanteles)

Distribution: England, Scotland 


\section{Glyptapanteles callidus (Haliday, 1834)}

Nomenclature:

Microgaster callidus Haliday, 1834

urolus (Papp, 1983, Apanteles)

Distribution: England, Scotland, Wales

Notes: Achterberg (1997) reinterpreted this name and treated majalis as the valid name for the species called callidus by Nixon (1973) and Papp (1983).

\section{Glyptapanteles compressiventris (Muesebeck, 1921)}

\section{Nomenclature:}

Apanteles compressiventris Muesebeck, 1921

liparidis misident.

Distribution: Scotland, Wales

Notes: Wilkinson (1945) dealt with the identity of the real liparidis, which does not occur in Britain or Ireland.

\section{Glyptapanteles fausta (Nixon, 1973)}

\section{Nomenclature:}

Apanteles fausta Nixon, 1973

Distribution: England, Scotland

Notes: Generic placement in doubt; some authors place both fausta and lateralis in Sathon. Papp (1983) synonymised fausta under Apanteles eugeni Papp, 1972 but later (Papp 1988) rescinded his action. Unfortunately Papp (1972) had chosen a non-reared specimen as holotype but a paratype of eugeni reared from Anthophila fabriciana (Linnaeus) is in BMNH and appears to be conspecific with fausta (described from this host). It seems probable that Papp's (Papp 1983) synonymy was justified but, as this requires further investigation, the name fausta is retained for now.

\section{Glyptapanteles fraternus (Reinhard, 1881)}

Nomenclature:

Apanteles fraternus Reinhard, 1881

Distribution: England 
Glyptapanteles fulvipes (Haliday, 1834)

Nomenclature:

Microgaster fulvipes Haliday, 1834

Distribution: England, Scotland, Wales, Ireland

\section{Glyptapanteles inclusus (Ratzeburg, 1844)}

Nomenclature:

Microgaster inclusus Ratzeburg, 1844

curvulus (Thomson, 1895, Microgaster)

rectinervis (Telenga, 1955, Apanteles)

Distribution: England

\section{Glyptapanteles lateralis (Haliday, 1834)}

Nomenclature:

Microgaster lateralis Haliday, 1834

Distribution: England, Scotland, Wales

Notes: Generic placement in doubt; some authors place both fausta and lateralis in Sathon.

\section{Glyptapanteles luciana (Nixon, 1973)}

\section{Nomenclature:}

Apanteles luciana Nixon, 1973

Distribution: Scotland

Notes: Described by Nixon (1973) partly from Scottish material but omitted by Huddleston (1978).

Glyptapanteles majalis (Wesmael, 1837)

Nomenclature:

Microgaster majalis Wesmael, 1837

callidus misident.

Distribution: England, Scotland

Notes: Achterberg (1997) treated majalis as the valid name for the species called callidus by Nixon (1973) and Papp (1983). 
Glyptapanteles menander (Nixon, 1973)

Nomenclature:

Apanteles menander Nixon, 1973

Distribution: Scotland

Glyptapanteles mygdonia (Nixon, 1973)

Nomenclature:

Apanteles mygdonia Nixon, 1973

Distribution: England, Scotland, Ireland

Glyptapanteles octonarius (Ratzeburg, 1852)

Nomenclature:

Microgaster octonarius Ratzeburg, 1852

stauropodis (Bridgman, 1889, Apanteles)

lucifugus (Lyle, 1917, Apanteles)

Distribution: England

Glyptapanteles pallipes (Reinhard, 1880)

Nomenclature:

Apanteles pallipes Reinhard, 1880

pallidipes (Marshall, 1885, Apanteles)

longicornis (Provancher, 1886, Microgaster)

radiatus (Ashmead, 1898, Apanteles)

reinhardi (Wilkinson, 1936, Apanteles)

Distribution: England, Scotland, Wales

Glyptapanteles pinicola (Lyle, 1917)

Nomenclature:

Apanteles pinicola Lyle, 1917

Distribution: England, Scotland 
Glyptapanteles porthetriae (Muesebeck, 1928)

Nomenclature:

Apanteles porthetriae Muesebeck, 1928

Distribution: England

Notes: added by Shaw and Skelton (2008)

Glyptapanteles salepus (Papp, 1983)

Nomenclature:

Apanteles salepus Papp, 1983

Distribution: England

Notes: added by Shaw (2012)

Glyptapanteles vitripennis (Curtis, 1830)

Nomenclature:

Microgaster vitripennis Curtis, 1830

fulcriger (Wesmael, 1837, Microgaster)

impavidus (Gautier \& Dresnyay, 1927, Apanteles)

Distribution: England, Scotland, Isle of Man

Genus Protapanteles Ashmead, 1898

Protapanteles anchisiades (Nixon, 1973)

Nomenclature:

Apanteles anchisiades Nixon, 1973

Distribution: England, Scotland, Wales, Isle of Man

Protapanteles endemus (Nixon, 1965)

Nomenclature:

Apanteles endemus Nixon, 1965

Distribution: England, Scotland 
Protapanteles enephes (Nixon, 1965)

Nomenclature:

Apanteles enephes Nixon, 1965

Distribution: England

Protapanteles hirtariae (Kotenko \& Tobias, 1986)

Nomenclature:

Apanteles hirtariae Kotenko \& Tobias, 1986

Distribution: Scotland

Notes: added by Shaw (2012)

Protapanteles immunis (Haliday, 1834)

Nomenclature:

Microgaster immunis Haliday, 1834

Distribution: England, Scotland, Wales, Ireland

Protapanteles incertus (Ruthe, 1859)

Nomenclature:

Microgaster incertus Ruthe, 1859

caberae (Marshall, 1885, Apanteles)

jugosus (Lyle, 1916, Apanteles)

mihalyii (Papp, 1973, Apanteles)

Distribution: England, Scotland, Isle of Man

Protapanteles parallelus (Lyle, 1917)

Nomenclature:

Apanteles parallelus Lyle, 1917

lylei (Shenefelt, 1972, Apanteles)

Distribution: England

Protapanteles popularis (Haliday, 1834)

Nomenclature:

Microgaster popularis Haliday, 1834 
Distribution: England, Scotland

Protapanteles triangulator (Wesmael, 1837)

Nomenclature:

Microgaster triangulator Wesmael, 1837

Distribution: England

Notes: Included in Protapanteles by Papp (1988) but Mason (1981) treated it as a species of Glyptapanteles. Nixon (1973) was unable to suggest close relatives.

Genus Rasivalva Mason, 1981

Rasivalva calceata (Haliday, 1834)

Nomenclature:

Microgaster calceatus Haliday, 1834

pubescens (Ratzeburg, 1844, Microgaster)

Distribution: England, Scotland

Rasivalva circumvecta (Lyle, 1918)

Nomenclature:

Diolcogaster circumvectus Lyle, 1918

Distribution: England, Scotland

Rasivalva marginata (Nees, 1834)

Nomenclature:

Microgaster marginatus Nees, 1834

Distribution: England, Scotland

Tribe Microgastrini Förster, 1863

Genus Hygroplitis Thomson, 1895

Hygroplitis pseudorussata Shaw, 1992

Distribution: England

Notes: added by Shaw (1992b) 


\section{Hygroplitis rugulosa (Nees, 1834)}

Nomenclature:

Microgaster rugulosus Nees, 1834

infumata (Haliday, 1834, Microgaster)

opaca (Ruthe, 1858, Microgaster)

Distribution: England, Wales, Ireland

Hygroplitis russata (Haliday, 1834)

Nomenclature:

Microgaster russatus Haliday, 1834

dimidiata (Wesmael, 1837, Microgaster)

basalis (Stephens, 1846, Microgaster)

aomoriensis (Matsumura, 1910, Microgaster)

Distribution: England, Scotland, Wales

Notes: Specimen from Islay in Hunterian Museum, Glasgow, det. MRS.

\section{Genus Iconella Mason, 1981}

Notes: species of Iconella excluded from the British and Irish list:

[lacteoides (Nixon, 1965, Apanteles); syn. memorabilis (Alexeev, 1971, Apanteles)]

[merula (Reinhard, 1880, Apanteles); syn. ?etiellae (Viereck, 1911, Apanteles)] Both of these species were mistakenly listed by Huddleston (1978); there is no evidence that they are British or Irish.

\section{Iconella aeola (Nixon, 1965)}

Nomenclature:

Apanteles aeolus Nixon, 1965

Distribution: England

Notes: added by Shaw (2012) 


\section{Genus Microgaster Latreille, 1804}

Nomenclature:

LIGANIRA Walker, 1860

LISSOGASTER Bengtsson, 1926

Notes: The current usage of the name Microgaster was restored by Opinion 1510 (ICZN 1988), after temporarily being applied to the genus here called Microplitis (with Microgaster as currently understood being referred to Lissogaster). This was an unfortunate name change as it coincided with Papp's (Papp 1976, Papp 1984) revisions of the genera, as well as several other important papers. Unless noted otherwise, distribution data taken from NMS, Nixon (1968) and Shaw (2012). Papp's (Papp 1976) subsequent revision of the genus added many species to Nixon's (Nixon 1968) revision, and Achterberg (1997) established the precedence of several Haliday names.

species of Microgaster excluded from the British and Irish list:

[auriculata (Fabricius, 1804, Ichneumon)] Listed as doubtfully British by Huddleston (1978) on the basis of Papp's (Papp 1976) listing of '?England'. No evidence that this is really a British or Irish species.

[deceptor Nixon, 1968] Listed as a British species by Huddleston (1978) in error; no evidence that this is a British or Irish species.

[fischeri Papp, 1960] British specimens, misidentified as fischeri by Nixon (1968), represented an undescribed species which was described by Shaw (2012) as raschkiellae.

[nobilis Reinhard, 1880; syn. compressifemur Fahringer, 1937] Listed as doubtfully British by Huddleston (1978) on the basis of Papp's (Papp 1976) listing of '?England'. No evidence that this is really a British or Irish species.

[postica Nees, 1834; syn. marginella Wesmael, 1837; ?ruficoxis Ruthe, 1858] Recorded as British by Marshall (1885), but probably in error as his diagnosis appears not to have been a Microgaster species in the modern sense. Papp's (Papp 1976) listing of England probably simply reflects Marshall's record, as does the listing in Huddleston (1978), and there is no evidence that this is a British or Irish species.

\section{Microgaster acilia Nixon, 1968}

Distribution: England

Notes: Raised from synonymy under meridiana (Papp 1999b) by Shaw (2012). 
Microgaster alebion Nixon, 1968

Distribution: England, Scotland

Notes: distribution data from Shaw (2004)

Microgaster areolaris Thomson, 1895

Distribution: England, Scotland

Microgaster arctostaphylica Shaw, 2012

Distribution: Scotland

Notes: added by Shaw (2012)

Microgaster consors Nixon, 1968

Distribution: England

Microgaster crassicornis Ruthe, 1860

Distribution: England

Microgaster ductilis Nixon, 1968

Distribution: England

Notes: added by Shaw (2012)

Microgaster fulvicrus Thomson, 1895

Nomenclature:

striatoscutellaris Kiss, 1927

Distribution: England, Scotland, Ireland, Isle of Man

Microgaster globata (Linnaeus, 1758)

Nomenclature:

Ichneumon globatus Linnaeus, 1758

laeviscuta Thomson, 1895

gossypina (Retzius, 1783, Ichneumon)

globator (Thunberg, 1824, Ichneumon)

anthomyiarum Bouché, 1834 
amentorum Ratzeburg, 1844

incurvata Papp, 1976; synonymy by Papp (2002)

Distribution: England, Scotland

Notes: Some distribution data from Papp (1976). It seems that laeviscuta has been included as a junior synonym of hospes in Fauna Europaea, with globata not referred to. Shaw (2012) notes that globata as perceived by Papp (1976) is almost certainly an aggregate.

\section{Microgaster hospes Marshall, 1885}

Nomenclature:

comptanae Viereck, 1911

Distribution: England, Scotland, Wales

\section{Microgaster luctuosa Haliday, 1834}

Nomenclature:

curvicrus Thomson, 1895; synonymy by Achterberg (1997)

Distribution: England, Scotland, Wales

\section{Microgaster meridiana Haliday, 1834}

\section{Nomenclature:}

spinolae Haliday, 1834 preocc.; synonymy by Achterberg (1997)

alexis Haliday, 1834 nom. nud.

grandis Thomson, 1895; synonymy by Achterberg (1997)

contubernalis Marshall, 1898

Distribution: England, Scotland, Wales, Ireland, Isle of Man

\section{Microgaster messoria Haliday, 1834}

Nomenclature:

tibialis Nees, 1834 preocc.; synonymy by Achterberg (1997)

vulgaris Ruthe, 1860

pluto Morley, 1936

Distribution: England, Scotland, Wales, Ireland 
Notes: Some Ruthe names traditionally regarded as synonyms of tibialis probably do not belong here Shaw (2012).

\section{Microgaster nigricans Nees, 1834}

Notes: A species of doubtful status. Curtis (1837) listed nigricans as a British species and Papp (1976), who stated that it was known only from the male, therefore listed it as English. Nixon (1968), however, did not deal with the name.

\section{Microgaster nixalebion Shaw, 2004}

Distribution: England, Wales

Notes: added by Shaw (2004)

\section{Microgaster novicia Marshall, 1885}

Nomenclature:

swammerdamiae Muesebeck, 1922

Distribution: England, Scotland

Microgaster opheltes Nixon, 1968

Distribution: Ireland

Microgaster pantographae Muesebeck, 1922

Distribution: England

Microgaster parvistriga Thomson, 1895

Distribution: England, Scotland

\section{Microgaster polita Marshall, 1885}

Nomenclature:

carinata Bengtsson, 1926 preocc.

bengtssoni Fahringer, 1937

Distribution: England, Scotland, Ireland 
Microgaster procera Ruthe, 1860

Nomenclature:

intermedia Ivanov, 1899

Distribution: Ireland

Microgaster raschkiellae Shaw, 2012

Nomenclature:

fischeri misident.

Distribution: England, Scotland, Wales

Notes: added by Shaw (2012)

Microgaster stictica Ruthe, 1858

Nomenclature:

confusa Papp, 1971

Distribution: England, Scotland, Wales, Isle of Man

Microgaster subcompleta Nees, 1834

Nomenclature:

annulipes Curtis, 1830

carinata Packard, 1881

Distribution: England, Scotland, Wales, Ireland, Isle of Man

Genus Paroplitis Mason, 1981

Paroplitis wesmaeli (Ruthe, 1860)

Nomenclature:

Microgaster wesmaeli Ruthe, 1860

picipes (Wesmael, 1837, Microgaster) preocc.

Distribution: England 
Genus Sathon Mason, 1981

Sathon falcatus (Nees, 1834)

Nomenclature:

Microgaster falcatus Nees, 1834

equestris (Haliday, 1834, Microgaster)

gladiator (Szépligeti, 1901, Apanteles)

Distribution: England, Scotland, Wales, Ireland

\section{Tribe Microplitini Mason, 1981}

\section{Genus Microplitis Förster, 1863}

Nomenclature:

DAPSILOTOMA Cameron, 1906

Notes: Unless noted otherwise, distribution data taken from Nixon (1970). Papp (1984) extensively revised the synonymy (as Microgaster species: unfortunately at that time Microplitis was briefly being regarded as a synonym of Microgaster, but this was subsequently rescinded by Opinion 1510 (ICZN 1988), whose conclusions are largely followed here.

species of Microplitis excluded from the British and Irish list:

[eremitus Reinhard, 1880] No evidence that this species has occurred in Britain or Ireland; probably listed in Shenefelt (1973) and Huddleston (1978) because Nixon (1970) reported its distribution as north-west European.

\section{Microplitis aduncus (Ruthe, 1860)}

Nomenclature:

Microgaster aduncus Ruthe, 1860

brachycerus (Thomson, 1895, Microgaster)

Distribution: Scotland

\section{Microplitis ? decens Tobias, 1964}

Distribution: Scotland

Notes: Added by Papp (1984) but it is unclear on what basis this species was listed as British (Shaw 2012). 


\section{Microplitis deprimator (Fabricius, 1798)}

\section{Nomenclature:}

Ichneumon deprimator Fabricius, 1798

ingratus (Haliday, 1834, Microgaster); synonymy by Achterberg (1997)

sordipes (Nees, 1834, Microgaster)

tau (Ratzeburg, 1852, Microgaster)

deprimatrix (Schulz, 1906, Microgaster)

Distribution: England, Scotland

Notes: Micropoltis sordipes is listed as a separate species in Taxapad (Yu et al. 2012) but we follow van Achterberg, in Fauna Europaea. However, the status of sordipes remains uncertain.

\section{Microplitis flavipalpis (Brullé, 1832)}

\section{Nomenclature:}

Microgaster flavipalpis Brullé, 1832

ruricola Lyle, 1918

Distribution: England

\section{Microplitis fordi Nixon, 1970}

Distribution: England, Scotland

Notes: Papp (1984) suggests that semicircularis (Ratzeburg, 1844, Microgaster) (type destroyed) may be a senior synonym.

\section{Microplitis fulvicornis (Wesmael, 1837)}

\section{Nomenclature:}

Microgaster fulvicornis Wesmael, 1837

calcarata misident.

pallidicornis Marshall, 1898

Distribution: England, Wales

\section{Microplitis impressus (Wesmael, 1837)}

Nomenclature:

Microgaster impressus Wesmael, 1837 
sispes Nixon, 1970

Distribution: England

Notes: added by Shaw (2012)

\section{Microplitis lugubris (Ruthe, 1860)}

Nomenclature:

Microgaster lugubris Ruthe, 1860

borealis Marshall, 1885

coracinus (Thomson, 1895, Microgaster)

rutheana Fahringer, 1937

Distribution: Scotland

\section{Microplitis malimbus (Papp, 1984)}

\section{Nomenclature:}

Microgaster malimba Papp, 1984

trochanterata misident

Distribution: England

Notes: Added by Shaw (2012). Nixon's (Nixon 1970) interpretation of trochanterata (not tuberculifer, of which trochanterata is a junior synonym) is actually referable to malimbus (Shaw 2012).

\section{Microplitis mandibularis Thomson, 1895}

Distribution: England, Scotland

\section{Microplitis mediator (Haliday, 1834)}

Nomenclature:

Microgaster mediator Haliday, 1834

medianus (Ruthe, 1860, Microgaster)

halidayi Fahringer, 1937

pseudomedianus Fahringer, 1937

Distribution: England, Scotland, Wales, Ireland, Isle of Man 
Microplitis moestus (Ratzeburg, 1852)

Nomenclature:

Microgaster moestus Ratzeburg, 1852

Microplitis naenia Nixon, 1970

Distribution: England

Microplitis ocellatae (Bouché, 1834)

Nomenclature:

Microgaster ocellatae Bouché, 1834

canaliculatus (Wesmael, 1837, Microgaster)

Distribution: England

Microplitis scrophulariae Szépligeti, 1898

Distribution: England

Notes: added by Shaw (2012)

Microplitis sofron Nixon, 1970

Nomenclature:

?stigmaticus (Ratzeburg, 1844, Microgaster)

Distribution: England, Scotland, Ireland

Notes: Papp (1984) suggests that stigmaticus may be a senior synonym.

Microplitis spectabilis (Haliday, 1834)

Nomenclature:

Microgaster spectabilis Haliday, 1834

fossulatus (Bouché, 1834, Microgaster)

?parvulus (Ruthe, 1860, Microgaster)

seuratii Marshall, 1898

testaceipes (Cameron, 1906, Dapsilotoma)

Distribution: England, Wales, Ireland 


\section{Microplitis spinolae (Nees, 1834)}

Nomenclature:

Microgaster spinolae Nees, 1834

sapporoensis Ashmead, 1906

radiorimatus Telenga, 1955

?quadridentatus (Provancher, 1886, Microgaster)

Distribution: England

Microplitis strenuus Reinhard, 1880

Nomenclature:

gracilis (Ruthe, 1860, Microgaster) preocc.

Distribution: England

Microplitis tristis (Nees, 1834)

Nomenclature:

Microgaster tristis Nees, 1834

dolens Marshall, 1885

Distribution: England, Scotland, Wales

Microplitis tuberculatus (Bouché, 1834)

Nomenclature:

Microgaster tuberculatus Bouché, 1834

fumipennis (Ratzeburg, 1852, Microgaster)

Distribution: England, Scotland, Ireland

\section{Microplitis tuberculifer (Wesmael, 1837)}

Nomenclature:

Microgaster tuberculifer Wesmael, 1837

calcaratus (Thomson, 1895, Microgaster)

trochanteratus (Thomson, 1895, Microgaster)

manevali Gautier \& Bonnamour, 1939

Distribution: England, Scotland 
Microplitis viduus (Ruthe, 1860)

Nomenclature:

Microgaster viduus Ruthe, 1860

Distribution: England

Microplitis xanthopus (Ruthe, 1860)

Nomenclature:

Microgaster xanthopus Ruthe, 1860

tenuipes (Thomson, 1895, Microgaster)

Distribution: Scotland, Wales, Ireland

\section{Microtypinae}

\section{Subfamily Microtypinae Szépligeti, 1908}

Notes: Belokobylskij et al. (2003) include Microtypus, without comment, in the Orgilinae, where it had traditionally been placed prior to recent phylogenetic work. In the molecular phylogeny of Belshaw and Quicke (2002) the genus Microtypus is the sister group to Homolobinae, as also found by Sharanowski et al. (2011) (albeit with this clade the sister group to Orgilinae). This relationship was originally suggested by Achterberg (1984b).

\section{Genus Microtypus Ratzeburg, 1848}

Nomenclature:

SIMILEARINUS Glowacki \& Karpiński, 1967

Notes: Nomenclature follows Capek and Achterberg (1992).

Microtypus wesmaelii Ratzeburg, 1848

Nomenclature:

dioryctriae Rohwer, 1920

Distribution: England

Notes: added by Shaw (1992a) 


\section{Miracinae}

Subfamily Miracinae Viereck, 1918

\section{Genus Mirax Haliday, 1833}

Nomenclature:

CENTISTIDEA Rohwer, 1914

Notes: There are also several unrecognised species in Britain.

\section{Mirax rufilabris Haliday, 1833}

\section{Nomenclature:}

spartii Haliday, 1835

dryochares Marshall, 1898

nanivorae Fischer, 1957

Distribution: England, Scotland

Notes: some distribution data from Shaw and Askew (1976) and NMS

\section{Opiinae}

\section{Subfamily Opiinae Blanchard, 1845}

Notes: The generic and tribal classification of opiines has been largely chaotic (Wharton 1988b). In Fauna Europaea, van Achterberg has enacted many taxonomic changes resulting from his work on Western Palaearctic Opiinae (in prep.), which are followed here. Some changes to the generic classification have been published by Wharton (1988b), van Achterberg (Achterberg 2004a, Achterberg 2004b, and in Belokobylskij et al. 2003) and by Li et al. (2013). Wharton (1988b) argued against recognition of tribes within Opiinae, except perhaps Ademonini (for the genus Ademon), with all the other genera in Opiini; van Achterberg (in prep.) does not recognise Ademonini. Some distribution data from Fischer (1958), Fischer (1967), Fischer (1997).

\section{Genus Ademon Haliday, 1833}

\section{Nomenclature:}

GIARDINAIA de Stefani-Perez, 1902

ANALOSTANIA Viereck, 1916 
Ademon decrescens (Nees, 1811)

Nomenclature:

Bracon decrescens Nees, 1811

mutuator (Nees, 1811, Bracon)

Distribution: England, Scotland, Wales, Ireland

Genus Apodesmia Förster, 1863

Nomenclature:

ALLOTYPUS Förster, 1863

LEMNAPHILOPIUS Fischer, 1972

AGNOPIUS Fischer, 1982

CRYPTOGNATHOPIUS Fischer, 1984

Notes: generic synonymy follows Li et al. (2013)

Apodesmia aemula (Haliday, 1836)

Nomenclature:

Opius aemulus Haliday, 1836

melba (Papp, 1978, Opius)

Distribution: England, Ireland

Apodesmia curvata (Fischer, 1957)

Nomenclature:

Opius curvatus Fischer, 1957

Distribution: England, Scotland

Notes: added by Godfray (1986)

Apodesmia irregularis (Wesmael, 1835)

Nomenclature:

Opius irregularis Wesmael, 1835

bipustulata (Fischer, 1958, Opius)

Distribution: England, Ireland 
Apodesmia ocellata (Wesmael, 1835)

Nomenclature:

Opius ocellatus Wesmael, 1835

areolaris (Thomson, 1895, Opius)

hungarica (Szépligeti, 1896, Opius)

bruta (Papp, 1978, Opius)

Distribution: England, Scotland

Apodesmia posticatae (Fischer, 1957)

Nomenclature:

Opius posticatae Fischer, 1957

seebensteinensis (Fischer, 1959, Opius)

hilaris (Fischer, 1963, Opius)

hostium (Fischer, 1964, Opius)

Distribution: England, Scotland

Apodesmia rufipes (Wesmael, 1835)

Nomenclature:

Opius rufipes Wesmael, 1835

taeniata Förster, 1863

taeniata (Fischer, 1957, Opius) preocc.

Distribution: England, Ireland

Apodesmia saeva (Haliday, 1837)

Nomenclature:

Opius saevus Haliday, 1837

Distribution: England, Scotland

Apodesmia saevula (Fischer, 1958)

Nomenclature:

Opius saevulus Fischer, 1958

Distribution: England 
Apodesmia similis (Szépligeti, 1898)

Nomenclature:

Opius similis Szépligeti, 1898

xylostei (Marshall, 1898, Opius)

similiformis (Fischer, 1957, Opius)

basirufa (Fischer, 1958, Opius)

nodata (Fischer, 1958, Opius)

periclymenii (Fischer, 1964, Opius)

altimontana (Fischer, 1969, Opius)

differens (Fischer, 1958, Opius)

parvipunctum (Fischer, 1958, Opius)

selkirkensis (Fischer, 2006, Opius); synonymy by van Achterberg (in prep.)

Distribution: England, Scotland, Ireland

Genus Atormus van Achterberg, 1998

\section{Atormus victus (Haliday, 1837)}

Nomenclature:

Opius victus Haliday, 1837

tarni (Papp, 1982, Opius)

Distribution: England, Scotland, Ireland

Notes: Listed as a synonym of Opius singularis in Huddleston (1978).

\section{Genus Bathystomus Förster, 1863}

Nomenclature:

COMPRESSARIA Königsmann, 1959

Notes: Although Wharton (1993) noted that the type (and only included) species of Compressaria is the same species as the type of Bathystomus, he did not formally synonymise these names and the synonymy has not been picked up on by, e.g. Belokobylskij et al. (2003) and Taxapad (Yu et al. 2012). Wharton (1988b) demonstrated that Bathystomus is a valid genus but Belokobylskij et al. (2003) treated it as a synonym of Diachasma. Van Achterberg (2014) formally synonymised Compressaria and pugnatrix. Huddleston (1978) included Compressaria pugnatrix as a species of Rogadinae. 
Bathystomus xanthopus Förster, 1863

Nomenclature:

pugnatrix (Marshall, 1895, Mesocrina)

compressiventris (Fischer, 1964, Opius); synonymy by van Achterberg (in prep.)

Distribution: England

\section{Genus Biophthora Förster, 1863}

Notes: Treated as a valid genus following Wharton (2006).

\section{Biophthora bajula (Haliday, 1837)}

Nomenclature:

Opius bajulus Haliday, 1837

beieri (Fischer, 1968, Sternaulopius); synonymy by Wharton (2006)

Distribution: England, Ireland

\section{Genus Biosteres Förster, 1863}

Nomenclature:

RHABDOSPILUS Förster, 1863

RHINOPLUS Förster, 1863

STENOSPILUS Förster, 1863

ZETETES Förster, 1863 preocc.

OPIELLUS Ashmead, 1900

CELIESTIELLA Cameron, 1903

\section{Biosteres analis (Wesmael, 1835)}

Nomenclature:

Opius analis Wesmael, 1835

colorativentris (Fischer, 1957, Opius)

Distribution: England, Scotland

Biosteres arenarius (Stelfox, 1959)

Nomenclature:

Opius arenarius Stelfox, 1959 
Distribution: Ireland

Biosteres bicolor Wesmael, 1835

Nomenclature:

vagator (Förster, 1863, Stenospilus)

Distribution: Ireland

Biosteres carbonarius (Nees, 1834)

Nomenclature:

Bracon carbonarius Nees, 1834

impressus (Wesmael, 1835, Opius)

procerus (Wesmael, 1835, Opius)

onzi (Fischer, 1959, Opius); synonymy by Achterberg (2014)

Distribution: England, Scotland, Wales, Ireland

Notes: Synonymised under carbonarius by Achterberg (1975), impressus was removed from synonymy by Fischer (1997), a move which is not accepted by van Achterberg (in prep.). Supporting van Achterberg's synonymy, Godfray (unpublished) has repeatedly reared specimens resembling both 'species' from collections of hosts made at the same place and time.

Biosteres haemorrhoeus (Haliday, 1837)

Nomenclature:

Opius haemorrhoeus Haliday, 1837

castaneiventris (Thomson, 1895, Opius)

palaearcticus Szépligeti, 1901

Distribution: England, Ireland

Biosteres magnicornis (Wesmael, 1835)

Nomenclature:

Opius magnicornis Wesmael, 1835

Distribution: England, Ireland 
Biosteres micans (Stelfox, 1957)

Nomenclature:

Opius micans Stelfox, 1957

nitidus (Stelfox, 1949, Opius) preocc.

Distribution: Scotland, Ireland

Biosteres placidus (Haliday, 1837)

Nomenclature:

Opius placidus Haliday, 1837

melanocerus (Wesmael, 1838, Opius)

tarsator (Thomson, 1895, Opius)

indotatus Viereck,1905

Distribution: England, Ireland

Biosteres rusticus (Haliday, 1837)

Nomenclature:

Opius rusticus Haliday, 1837

Distribution: England, Scotland, Ireland

Biosteres scabriculus (Wesmael, 1835)

Nomenclature:

Opius scabriculus Wesmael, 1835

Distribution: Ireland

Biosteres spinaciae (Thomson, 1895)

Nomenclature:

Opius spinaciae Thomson, 1895

pegomyiae (Gahan, 1917, Opius)

hyoscyamiellus (Viereck, 1925, Opius)

Distribution: England

Notes: added by Godfray (1988) 
Biosteres sylvaticus (Haliday, 1837)

Nomenclature:

Opius sylvaticus Haliday, 1837

clypealis (Thomson, 1895, Opius)

nitidus Szépligeti, 1896

Distribution: England, Scotland, Ireland

Biosteres wesmaelii (Haliday, 1837)

Nomenclature:

Opius wesmaelii Haliday, 1837

carbonarius (Wesmael, 1835, Opius) preocc.

ultor (Förster, 1863, Zetetes)

ultor (Fischer, 1957, Opius) preocc.

jonaitisi (Jakimavi?ius, 1977, Opius)

Distribution: England, Scotland

Genus Bitomoides van Achterberg, 2004

Bitomoides rugosus (Wesmael, 1838)

Nomenclature:

Opius rugosus Wesmael, 1838

rugiventris (Thomson, 1895, Opius); synonymy by Achterberg (2014)

Distribution: England

Notes: Listed as British by Kloet and Hincks (1945) but omitted by Huddleston (1978).

Material in NMS has been identified by van Achterberg.

Genus Chilotrichia Förster, 1863

Nomenclature:

TRICHOPIUS Thomson, 1895

Chilotrichia blanda (Haliday, 1837)

Nomenclature:

Opius blandus Haliday, 1837 
Distribution: England, Ireland

\section{Genus Desmiostoma Förster, 1863}

Notes: Regarded by Wharton (Wharton 1983, Wharton 1988b) as a junior synonym of Opius.

Desmiostoma parvulum (Wesmael, 1835)

Nomenclature:

Opius parvulus Wesmael, 1835

nudiscutum (Fischer, 1964, Opius)

ziratus (Papp, 1982, Opius)

temporale (Fischer, 1958, Opius)

Distribution: England, Scotland

\section{Genus Diachasma Förster, 1863}

Nomenclature:

Atoreuteus Förster, 1863

Lytacra Förster, 1863

Alysopius Tobias, 1976

Diachasma caffer (Wesmael, 1835)

Nomenclature:

Opius caffer Wesmael, 1835

stygium (Förster, 1863, Lytacra)

Diachasma cephalotes (Wesmael, 1835)

Nomenclature:

Opius cephalotes Wesmael, 1835

Distribution: England

Diachasma fulgidum (Haliday, 1837)

Nomenclature:

Opius fulgidus Haliday, 1837

Distribution: England, Ireland 
Genus Eurytenes Förster, 1863

Subgenus Eurytenes Förster, 1863

Eurytenes (Eurytenes) abnormis (Wesmael, 1835)

Nomenclature:

Opius abnormis Wesmael, 1835

Distribution: England, Ireland

Notes: According to Walker and Wharton (2011), Opius paradoxus Ratzeburg, 1848, sometimes treated as a valid name (synonymous with abnormis) should be regarded as invalid as it was first proposed as a synonym of abnormis; Walker and Wharton (2011) also provide some locality data.

Eurytenes (Eurytenes) britannicola Fischer, 2006

Distribution: England

Notes: Added by Fischer (2006): mis-placed in Eurytenes (van Achterberg, pers. comm.).

\section{Subgenus Stigmatopoea Fischer, 1986}

Notes: The generic name Stigmatopoea has been treated as a synonym of Xynobius by Achterberg (2004a) but the type species, macrocerus, has been regarded as a species of Eurytenes, a genus which was ignored by Achterberg (2004a). Both Xynobius and Stigmatopoea are treated as subgenera of Eurytenes, following Wharton (2006), although Li et al. (2013) again synonymised Stigmatopoea with Xynobius.

Eurytenes (Stigmatopoea) macrocerus (Thomson, 1895)

Nomenclature:

Opius macrocerus Thomson, 1895

hians (Stelfox, 1949, Opius)

Distribution: England, Scotland, Ireland

\section{Subgenus Xynobius Förster, 1863}

Nomenclature:

ACLISIS Förster, 1863

HOLCONOTUS Förster, 1863 
AULONOTUS Ashmead, 1900

ERISTERNAULAX Viereck, 1914

XYNOBIOTENES Fischer, 1998

Notes: Generic synonymy from Achterberg (2004a) and Li et al. (2013) but treated as a subgenus of Eurytenes by Wharton (2006).

Eurytenes (Xynobius) aciculatus (Thomson, 1895)

Nomenclature:

Opius aciculatus Thomson, 1895

tenuicornis (Thomson, 1895, Opius); synonymy by van Achterberg (in prep.)

Distribution: Ireland

Eurytenes (Xynobius) aemuloides (Fischer, 1958)

Nomenclature:

Opius aemuloides Fischer, 1958

Distribution: England

Eurytenes (Xynobius) caelatus (Haliday, 1837)

Nomenclature:

Opius caelatus Haliday, 1837

isomera (Förster, 1863, Aclisis)

pallipes Förster, 1863

pallidipes Dalla Torre, 1898

Distribution: England, Scotland, Ireland

Eurytenes (Xynobius) comatus (Wesmael, 1835)

Nomenclature:

Opius comatus Wesmael, 1835

sulcifer (Papp, 1967, Dapsilarthra)

Distribution: England, Ireland 
Eurytenes (Xynobius) geniculatus (Thomson, 1895)

Nomenclature:

Opius geniculatus Thomson, 1895

albicoxis (Marshall, 1898, Opius)

Distribution: England, Scotland

Eurytenes (Xynobius) holconotus (Fischer, 1958)

Nomenclature:

Opius holconotus Fischer, 1958

Distribution: England

Eurytenes (Xynobius) maculipes Wesmael, 1835

Nomenclature:

addendus Fischer, 1959

turcmenicus Fischer, 1959

Distribution: England, Ireland

Eurytenes (Xynobius) polyzonius (Wesmael, 1835)

Nomenclature:

Opius polyzonius Wesmael, 1835

Distribution: England, Scotland

Eurytenes (Xynobius) silenis (Fischer, 1967)

Nomenclature:

Diachasma silenis Fischer, 1967

Distribution: Wales

Notes: Described from Welsh material (Fischer 1967) but omitted by Huddleston (1978).

Eurytenes (Xynobius) thomsoni (Fischer, 1971)

Nomenclature:

Opius thomsoni Fischer, 1971

annulicornis (Thomson, 1895, Opius) preocc. 
Distribution: England

Eurytenes (Xynobius) sp. A van Achterberg, in prep.

Distribution: England

Notes: reared by Godfray, in NMS

Genus Neopius Gahan, 1917

Notes: Raised from synonymy with Phaedrotoma by Li et al. (2013).

Neopius rudis (Wesmael, 1835)

Nomenclature:

Opius rudis Wesmael, 1835

carinaticeps Gahan, 1917

Distribution: England, Ireland

Genus Opiognathus Fischer, 1972

Notes: Raised from synonymy with Phaedrotoma by Li et al. (2013).

Opiognathus pactus (Haliday, 1837)

Nomenclature:

Opius pactus Haliday, 1837

Distribution: England, Ireland

\section{Genus Opiostomus Fischer, 1972}

Nomenclature:

SNOFLAKOPIUS Fischer, 1972

JUCUNDOPIUS Fischer, 1984

OETZALOTENES Fischer, 1998

OPIOTENES Fischer, 1998

Notes: Raised from synonymy with Opius by Li et al. (2013), who report the generic synonymy. 
Opiostomus aureliae (Fischer, 1957)

Nomenclature:

Opius aureliae Fischer, 1957

Distribution: England, Scotland

Notes: added by Godfray and Achterberg (2015)

Opiostomus campanariae (Fischer, 1959)

Nomenclature:

Opius campanariae Fischer, 1959

Distribution: Scotland

Notes: added by Godfray and Achterberg (2015)

Opiostomus griffithsi (Fischer, 1962)

Nomenclature:

Opius griffithsi Fischer, 1962

Distribution: England

Opiostomus leptostigma (Wesmael, 1835)

Nomenclature:

Opius leptostigma Wesmael, 1835

percontator (Fischer, 1964, Opius)

Distribution: England, Scotland

Notes: Recorded as a British species by various authors, latterly by Fischer (1967), and reared recently by Godfray (unpublished), but omitted by Huddleston (1978).

\section{Genus Opius Wesmael, 1835}

Nomenclature:

CRYPTONASTES Förster, 1863

HYPOCYNODUS Förster, 1863

HYPOLABIS Förster, 1863

MISOPHTHORA Förster, 1863

DESMATOPHORUS Thomson, 1895 

ALLOPHLEBUS Fischer, 1972
NOSOPAEOPIUS Fischer, 1972
OPIOTHORAX Fischer, 1972
PENDOPIUS Fischer, 1972
STOMOSEMA Fischer, 1972
ODONTOPOEA Fischer, 1987

Notes: Restricted by Achterberg and Salvo (1997) to a group of species with distinctive mandibles.

\section{Opius agromyzicola Fischer, 1967}

Distribution: England

\section{Opius ambiguus Wesmael, 1835}

Nomenclature:

celsus Haliday, 1837; synonymy by Achterberg (2014)

longipes Fischer, 1957

phytomyzae Fischer, 1957

Distribution: England, Scotland, Ireland

\section{Opius brevipalpis Thomson, 1895}

Nomenclature:

mutus Fischer, 1964

gyoerfii Fischer, 1958; synonymy by Achterberg (2014)

Distribution: England

\section{Opius caudifer Fischer, 1958}

\section{Nomenclature:}

longicornis misident.

Distribution: England

Notes: Listed as a synonym of longicornis Thomson, 1895, in Taxapad (Yu et al. 2012), regarded as a separate, valid species by van Achterberg (in prep.). Recorded as British by Fischer (1967) as longicornis but omitted by Huddleston (1978). 
Opius cingulatus Wesmael, 1835

Nomenclature:

dentifer Thomson, 1895

stramineipes Thomson, 1895

Opius compar Marshall, 1894

Nomenclature:

pulchrithorax Fischer, 1958

Opius crassipes Wesmael, 1835

Distribution: England

Opius flammeus Fischer, 1959

Distribution: England

Opius funebris Wesmael, 1835

Nomenclature:

latipes misident.

Distribution: England, Ireland

Notes: Listed as a synonym of pygmaeator in Taxapad (Yu et al. 2012), regarded as a separate, valid species (=latipes sensu Fischer) by van Achterberg (in prep.).

Opius fuscipennis Wesmael, 1835

Distribution: England

Notes: Fischer (1960) mistakenly listed this species as fuscipennis (Szépligeti, 1914, Rhinoplus) (van Achterberg, pers. comm.), a separate species now placed in the genus Pseudorhinoplus Fischer, 1972.

Opius gracilis Fischer, 1957

Nomenclature:

csikii Fischer, 1957

minor Fischer, 1957

nigrithorax Fischer, 1958 preocc.

Distribution: England 
Opius instabilis Wesmael, 1835

Opius levis Wesmael, 1835

Nomenclature:

apiculator (Nees, 1834, Bracon)

filicornis Thomson, 1895

varipes Szépligeti, 1898

Distribution: England, Wales, Ireland

Notes: Opius filicornis was recorded as new to Britain by Godfray (1986) but synonymised with levis by van Achterberg (in Belokobylskij et al. 2003).

Opius lucidus Szépligeti, 1896

Distribution: England, Scotland

\section{Opius lugens Haliday, 1837}

Nomenclature:

abscissus Thomson, 1895

obscurus Szépligeti, 1901

adveniens Fischer, 1960

Distribution: England, Ireland

Opius nigricoloratus Fischer, 1958

Nomenclature:

dureseaui Fischer, 1975; synonymy by Achterberg (2014)

Distribution: Scotland, Wales

Notes: added by Godfray and Achterberg (2015)

Opius ochrogaster Wesmael, 1835

Nomenclature:

nigriceps Szépligeti, 1898

neopusillus Fischer, 1957

Distribution: England, Scotland, Ireland 
Opius orbiculator (Nees, 1811)

Nomenclature:

Bracon orbiculator Nees, 1811

breviscapus Thomson, 1895

Distribution: Ireland

\section{Opius pallipes Wesmael, 1835}

Nomenclature:

exilis Haliday, 1837; synonymy by Achterberg (1997)

pallidipes (Marshall, 1872, Hypolabis)

liopleuris Thomson, 1895

piceus Thomson, 1895

adaequator (Fischer, 1964, Hypolabis) nom. nud.

lividipes (Fischer, 1964, Hypolabis) nom. nud.

subsulcatus (Fischer, 1964, Hypolabis) nom. nud.

extusus Papp, 1981

cisromensis Papp, 1982

Distribution: England, Scotland, Wales, Ireland

Opius pendulus Haliday, 1837

Nomenclature:

latipes Fischer, 1958

Distribution: England, Ireland

Opius phytobiae Fischer, 1959

Distribution: England

Notes: added by Godfray (1986)

Opius propodealis Fischer, 1958

Distribution: England 
Opius pygmaeator (Nees, 1811)

Nomenclature:

Bracon pygmaeator Nees, 1811

ruminans Fischer, 1957

dilatatus Fischer, 1960

meracus Fischer, 1960

Distribution: England, Ireland

Opius pygmaeus Fischer, 1962

Distribution: England

Opius singularis Wesmael, 1835

Nomenclature:

clarus Haliday, 1836; synonymy by Fischer (1997)

spretus Haliday, 1836; synonymy by Fischer (1997)

vindex Haliday, 1837; synonymy by van Achterberg (in prep.)

arenosus Szépligeti, 1898

Distribution: England, Ireland

Notes: Both clarus and spretus were also synonymised by Achterberg (1997) but Fischer's publication pre-dated this.

\section{Opius soenderupianus Fischer, 1967}

Distribution: England

Notes: added by Godfray and Achterberg (2015)

Opius tenellae Fischer, 1969

Distribution: Scotland, Ireland

\section{Genus Phaedrotoma Förster, 1863}

Nomenclature:

EUTRICHOPSIS Förster, 1863

NOSOPOEA Förster, 1863

TOLBIA Cameron, 1907 
BRACHYCENTRUS Szépligeti, 1907

COELOREUTEUS Roman, 1910

HEXAULAX Cameron, 1910

BAEOCENTRUM Schulz, 1911

NEODIOSPILUS Szépligeti, 1911

NEOPIUS Fischer, 1965

EUOPIUS Fischer, 1967

GASTROSEMA Fischer, 1972

GERIUS Fischer, 1972

GRIMNIRUS Fischer, 1972

HOENIRUS Fischer, 1972

MEROTRACHYS Fischer, 1972

MIMIRUS Fischer, 1972

PHLEBOSEMA Fischer, 1972

NEOEPHEDRUS Samanta, Tamili, Saha \& Raychaudhuri, 1983

ADONTOPIUS Fischer, 1984

KAINOPAEOPIUS Fischer, 1987

MILLENIOPIUS Fischer, 1996

NEOTROPOPIUS Fischer, 1999

Notes: Phaedrotoma was raised from synonymy with Opius by Achterberg and Salvo (1997) to accommodate many species previously placed in Opius. Generic synonymy follows Li et al. (2013).

species of Phaedrotoma excluded from the British and Irish list:

[viennensis (Fischer, 1959, Opius)] Listed by Huddleston (1978) but we cannot trace any published records or specimens.

\section{Phaedrotoma aethiops (Haliday, 1837)}

\section{Nomenclature:}

Opius aethiops Haliday, 1837

Distribution: England, Ireland 
Phaedrotoma caesa (Haliday, 1837)

Nomenclature:

Opius caesus Haliday, 1837

punctiventris (Thomson, 1895, Opius)

subtilis (Szépligeti, 1898, Opius)

hydrelliae (Rimsky-Korsakov, 1925, Opius)

hydrelliae (Muesebeck, 1933, Opius) preocc.

aquatica (Muesebeck, 1967, Opius)

hydrelliana (Fischer, 1971, Opius)

Distribution: England, Ireland

Phaedrotoma curvata (Fischer, 1957)

Nomenclature:

Opius curvatus Fischer, 1957

Distribution: Scotland

Notes: added by Godfray (1986)

Phaedrotoma decorata (Stelfox, 1949)

Nomenclature:

Opius decoratus Stelfox, 1949

Distribution: Ireland

Phaedrotoma depeculator (Förster, 1863)

Nomenclature:

Opius depeculator Förster, 1863

semiaciculata (Stelfox, 1949, Opius)

Distribution: England, Ireland

Phaedrotoma diversa (Szépligeti, 1898)

Nomenclature:

Opius diversus Szépligeti, 1898

Distribution: England 
Notes: Recorded as British by Fischer (1960) but omitted by Huddleston (1978).

Phaedrotoma exigua (Wesmael, 1835)

Nomenclature:

Opius exiguus Wesmael, 1835

Distribution: England, Ireland

Phaedrotoma fallax (Szépligeti, 1896)

Nomenclature:

Opius fallax Szépligeti, 1896

Distribution: England

Notes: Listed as a synonym of Opius instabilis in Taxapad (Yu et al. 2012) but regarded as a valid species, in Phaedrotoma, by van Achterberg (in prep.).

Phaedrotoma fasciata (Thomson, 1895)

Nomenclature:

Opius fasciatus Thomson, 1895

comparanda (Fischer, 1958, Opius)

Distribution: England

Phaedrotoma heringi (Fischer, 1962)

Nomenclature:

Opius heringi Fischer, 1962

Distribution: Ireland

Phaedrotoma instabiloides (Fischer, 1959)

Nomenclature:

Opius instabiloides Fischer, 1959

Distribution: Ireland

Phaedrotoma minusculae (Fischer, 1967)

Nomenclature:

Opius minusculae Fischer, 1967 
Distribution: England

Phaedrotoma monticola (Szépligeti, 1898)

Nomenclature:

Opius monticola Szépligeti, 1898

Distribution: England

Notes: added by Fischer (1997)

Phaedrotoma munda (Förster, 1863)

Nomenclature:

Eutrichopsis munda Förster, 1863

munda (Fischer, 1957, Opius) preocc.

Distribution: England

Phaedrotoma nitidulator (Nees, 1834)

Nomenclature:

Opius nitidulator Nees, 1834

vittata (Ruschka, 1915, Opius) preocc.

Phaedrotoma novojariae (Fischer, 2006)

Nomenclature:

Opius novojariae Fischer, 2006

stigmatocauda (Fischer, 2006, Eurytenes); synonymy by van Achterberg (in prep.)

Distribution: England

Notes: added by Fischer (2006)

Phaedrotoma paraphytomyzae (Fischer, 2005)

Nomenclature:

Opius paraphytomyzae Fischer, 2005

Distribution: England

Notes: added by Fischer (2005) 
Phaedrotoma pulchriceps (Szépligeti, 1898)

Nomenclature:

Opius pulchriceps Szépligeti, 1898

ilicis (Nixon, 1939, Opius)

pulcherrimus (Fischer, 1958, Opius)

pulchriventris (Fischer, 1958, Opius); synonymy by Achterberg (2014)

vexator (Fischer, 1964, Opius)

affectus (Papp, 1981, Opius)

Distribution: England, Ireland

\section{Phaedrotoma recondes van Achterberg, 2004}

Nomenclature:

reconditor misident.

Notes: Added by van Achterberg in Fauna Europaea; described by van Achterberg because the material named Opius reconditor (now classified in Rhogadopsis) by authors (e.g. Fischer 1972, Fischer and Koponen 1999) is not conspecific with the type. Both species apparently occur in Britain.

Phaedrotoma reptantis (Fischer, 1957)

Nomenclature:

Opius reptantis Fischer, 1957

Distribution: England, Scotland

Notes: Listed as a synonym of Opius ambiguus in Taxapad (Yu et al. 2012) but regarded as a valid species, in Phaedrotoma, by van Achterberg (in prep.).

\section{Phaedrotoma rex (Fischer, 1958)}

Nomenclature:

Opius rex Fischer, 1958

Distribution: England

\section{Phaedrotoma rudiformis (Fischer, 1958)}

\section{Nomenclature:}

Opius rudiformis Fischer, 1958

uligiloci (Fischer, 2006, Opius); synonymy by Achterberg (2014) 
Distribution: England

Phaedrotoma staryi Fischer, 1958

Nomenclature:

sitagrus Papp, 1982

Distribution: England, Scotland

Phaedrotoma tacita (Haliday, 1837)

Nomenclature:

Opius tacitus Haliday, 1837

Distribution: England

Notes: omitted by Huddleston (1978)

Phaedrotoma variegata (Szepligeti, 1896)

Nomenclature:

Opius variegatus Szepligeti, 1896

Distribution: England, Scotland, Ireland

\section{Genus Rhogadopsis Brèthes, 1913}

Nomenclature:

LISSOSEMA Fischer, 1972

Notes: Raised from synonymy with Phaedrotoma by Li et al. (2013).

Rhogadopsis reconditor (Wesmael, 1835)

Nomenclature:

Opius reconditor Wesmael, 1835

docilis (Haliday, 1837, Opius)

parvungula Thomson, 1895

Distribution: England, Ireland

Notes: Opius docilis was synonymised with reconditor by Achterberg (1997) but listed as a separate species in Taxapad (Yu et al. 2012); re-synonymised by Achterberg (2014). 


\section{Genus Utetes Förster, 1863}

Nomenclature:

THEROBOLUS Förster, 1863

FREKIUS Fischer, 1971; synonymy by Wharton (2006)

Notes: species of Utetes excluded from the British and Irish list:

[christenseni (Papp, 1982, Opius)] Listed in Fauna Europaea as occurring in Britain, but presumably mistakenly as no literature or specimen records can be located. Known only from Georgia, Greece and Ukraine (data from Yu et al. 2012).

\section{Utetes caudatus (Wesmael, 1835)}

Nomenclature:

Opius caudatus Wesmael, 1835

exsertus (Thomson, 1895, Opius)

Distribution: England

Notes: Listed as English by Fischer and Koponen (1999), presumably following Fischer (1958), but not listed in Huddleston (1978).

\section{Utetes coracinus (Thomson, 1895)}

\section{Nomenclature:}

Opius coracinus Thomson, 1895

Distribution: England

Notes: BMNH, det. Fischer, added in Fauna Europaea; there may be a literature citation for its occurrence in Britain (van Achterberg, pers. comm.) but we have been unable to trace it.

\section{Utetes fulvicollis (Thomson, 1895)}

\section{Nomenclature:}

Opius fulvicollis Thomson, 1895

cupidus (Gahan, 1919, Opius)

Distribution: England

Notes: added by Godfray (1986) 
Utetes rotundiventris (Thomson, 1895)

Nomenclature:

Opius rotundiventris Thomson, 1895

Distribution: England

Utetes ruficeps (Wesmael, 1835)

Nomenclature:

Opius ruficeps Wesmael, 1835

Distribution: England

Utetes testaceus (Wesmael, 1838)

Nomenclature:

Opius testaceus Wesmael, 1838

Distribution: England

Utetes truncatus (Wesmael, 1838)

Nomenclature:

Opius truncatus Wesmael, 1838

Distribution: England

Utetes zelotes (Marshall, 1891)

Nomenclature:

Opius zelotes Marshall, 1891

insertus (Fischer, 1971, Opius)

incertus misspelling

Distribution: England, Scotland 


\section{Orgilinae}

Subfamily Orgilinae Ashmead, 1900

Genus Orgilus Haliday, 1833

Nomenclature:

ISCHIUS Wesmael, 1837

MACROPALPUS Ratzeburg, 1844

ORESIMUS Ashmead, 1900

ORGILOMORPHA Ashmead, 1900

ISCHIOLUS Hellén, 1958

Notes: Taxonomic and much distribution data from Taeger (1989).

Orgilus achterbergi Taeger, 1989

Distribution: England

Notes: added by Taeger (1989)

Orgilus dovnari Tobias, 1986

Nomenclature:

ukrainicus Tobias, 1986

Distribution: England

Notes: NMS, det. Taeger, added here

Orgilus interjectus Taeger, 1989

Distribution: England

Notes: added by Taeger (1989)

Orgilus ischnus Marshall, 1898

Nomenclature:

subtilirugosus Papp, 1971

Distribution: England, Wales 


\section{Orgilus leptocephalus Hartig, 1838}

\section{Nomenclature:}

obscurator misident.

rugulosus Fahringer, 1937

hyperboreus Hellén, 1958

Distribution: Ireland

Notes: Although Taeger (1989) clarified the identity of obscurator auctt. as leptocephalus, this species was recorded as obscurator by O'Connor et al. (1999).

\section{Orgilus minor Taeger, 1989}

Distribution: England

Notes: NMS, det. Taeger, added here

Orgilus parvipennis Thomson, 1895

Nomenclature:

micropterus Morley, 1907

macroptera (Rudow, 1917, Aptesis)

decoratus Hellén, 1946

curtipennis Fischer, 1958

discolor Hellén, 1958

Distribution: England, Scotland

\section{Orgilus pimpinellae Niezabitowski, 1910}

Nomenclature:

laevigator misident.

Distribution: England, Scotland, Ireland

Notes: Added by Taeger (1989); apparently recorded as Irish under the name Microdus laevigator Nees, 1812 (O'Connor et al. 1999), which is listed as a species inquirendae by Taeger (1989).

\section{Orgilus punctulator (Nees, 1811)}

\section{Nomenclature:}


rufiventris Fahringer, 1937

Distribution: England

Notes: added by Taeger (1989)

Orgilus rugosus (Nees, 1834)

Nomenclature:

Microgaster rugosus Nees, 1834

Distribution: England

Notes: NMS, det. Taeger, added here

Orgilus tobiasi Taeger, 1989

Distribution: Wales, Ireland

Notes: added by Taeger (1989)

\section{Pambolinae}

Subfamily Pambolinae Marshall, 1885

Tribe Chremylini Hellén, 1957

Genus Chremylus Haliday, 1833

Nomenclature:

PENECERUS Wesmael, 1838

PARAMESOCRINA Nagamori, 1925

\section{Chremylus elaphus Haliday, 1833}

Nomenclature:

rubiginosus (Nees, 1834, Hormius)

transversus (Say, 1836, Bracon)

nigriceps Ashmead, 1893

terminalis Ashmead, 1893

japonicus Ashmead, 1896

tineavorus (Nagamori, 1925, Paramesocrina) 
Distribution: England, Ireland

Tribe Pambolini Marshall, 1885

Genus Dimeris Ruthe, 1854

Nomenclature:

PARAPTESIS Magretti, 1884

Dimeris mira Ruthe, 1854

Nomenclature:

melanocephala (Marshall, 1870, Pambolus)

flavipes (Magretti, 1884, Paraptesis)

aptera Marshall, 1885

inermis Fitch, 1885

Distribution: England

Genus Pambolus Haliday, 1836

Subgenus Phaenodus Förster, 1863

Pambolus (Phaenodus) pallipes (Förster, 1863)

Nomenclature:

Phaenodus pallipes Förster, 1863

flavipes (Förster, 1863, Araphis)

pallidipes (Marshall, 1897, Phaenodus)

chalveri (Docavo, 1960, Phaenodus)

Distribution: England, Scotland

Notes: added by Belokobylskij (1986)

\section{Subgenus Pambolus Haliday, 1836}

Nomenclature:

ARAPHIS Ruthe, 1854

ARRHAPHIS misspelling

ARHAPHIS misspelling 
FOLCHINIA Kieffer, 1906

PARAMBOLUS Dahl, 1912

Notes: species of Pambolus (Pambolus) excluded from the British and Irish list:

[biglumis (Haliday, 1836, Rogas); syn. rosenhaueri (Ratzeburg, 1852, Pezomachus); dubius (Fitch, 1885, Araphis); imminens (Fitch, 1885, Araphis)] Included as British in Fa una Europaea and Taxapad (Yu et al. 2012) (but not by Huddleston 1978); this seems to be on the basis of Fitch's descriptions of the synonymous names dubius and imminens in Marshall's (Marshall 1885) monograph of British Braconidae. However, the types of Fitch's species are from the Ruthe collection of German material and we have seen no British or Irish specimens of biglumis.

\section{Rhysipolinae}

Subfamily Rhysipolinae Belokobylskij, 1984

Genus Pseudavga Tobias, 1964

Pseudavga flavicoxa Tobias, 1964

Nomenclature:

rustus (Papp, 1991, Rhysipolis)

Distribution: England

Notes: added by Shaw and Sims (2015)

\section{Genus Rhysipolis Förster, 1863}

Notes: Most synonymy has been omitted; published taxonomy has been confused and there are several more species present than have been recorded in the literature, with the application of names not yet settled.

\section{Rhysipolis decorator (Haliday, 1836)}

Nomenclature:

Rogas decorator Haliday, 1836

ruficeps (Wesmael, 1838, Exothecus)

ruficornis (Szépligeti, 1896, Xenarcha)

Distribution: England, Scotland, Isle of Man 
Notes: some distribution data from Shaw and Askew (1976)

\section{Rhysipolis hariolator (Haliday, 1836)}

Nomenclature:

Rogas hariolator Haliday, 1836

barbatus (Wesmael, 1838, Exothecus)

Distribution: England, Scotland

Notes: some distribution data from Shaw and Askew (1976)

Rhysipolis meditator (Haliday, 1836)

Nomenclature:

Rogas meditator Haliday, 1836

Distribution: England, Scotland

\section{Rhysipolis variabilis (Szépligeti, 1896)}

Nomenclature:

Xenarcha variabilis Szépligeti, 1896

Distribution: England

Notes: NMS, det. Shaw \& van Achterberg, added here; treated as a synonym of meditator by Belokobylskij and Tobias (1986) and Belokobylskij et al. (2003) but here treated as a valid species.

\section{Rhysipolis varicoxa (Thomson, 1892)}

Nomenclature:

Exothecus varicoxa Thomson, 1892

Distribution: England

Notes: NMS, det. Shaw \& van Achterberg, added here; treated as a synonym of meditator by Belokobylskij and Tobias (1986) and Belokobylskij et al. (2003) but here treated as a valid species. 


\section{Rhyssalinae}

Subfamily Rhyssalinae Förster, 1863

Nomenclature:

HISTEROMERINAE Fahringer, 1930

Tribe Acrisidini Hellén, 1957

Genus Acrisis Förster, 1863

Nomenclature:

EUCHASMUS Marshall, 1888

EPISIGALPHUS Ashmead, 1900

Acrisis exiguus (Marshall, 1888)

Nomenclature:

Euchasmus exiguus Marshall, 1888

Distribution: England

Genus Proacrisis Tobias, 1983

Notes: Treated as a synonym of Acrisis in Fauna Europaea, as a separate genus by Belokobylskij et al. (2003).

Proacrisis acutus Tobias, 1983

Distribution: England

Notes: NMS, det. Shaw \& van Achterberg, added here

Proacrisis rarus Tobias, 1983

Distribution: England

Notes: NMS, det. Shaw \& van Achterberg, added on Fauna Europaea 


\title{
Tribe Histeromerini Fahringer, 1930
}

Notes: Zaldívar-Riverón et al. (2006) and Sharanowski et al. (2011) both found, on the basis of molecular phylogenetic results, that the morphologically and biologically aberrant genus Histeromerus belongs in Rhyssalinae; however, there is as yet no indication as to how Histeromerus can be accommodated within the existing tribal classification of Rhyssalinae, so we simply use Histeromerini for now.

\section{Genus Histeromerus Wesmael, 1838}

Nomenclature:

MITHOTYNIA Hedqvist, 1976

\section{Histeromerus mystacinus Wesmael, 1838}

Nomenclature:

apterus (Hedqvist, 1976, Mithotynia)

Distribution: England, Wales, Ireland

Notes: distribution data from Shaw (1995) and the Horniman Museum

\section{Tribe Rhyssalini Förster, 1863}

\section{Genus Dolopsidea Hincks, 1944}

Nomenclature:

DOLOPS Marshall, 1889 preocc.

EXONTSIRA Belokobylskij, 1982

\section{Dolopsidea indagator (Haliday, 1836)}

\section{Nomenclature:}

\author{
Rogas indagator Haliday, 1836 \\ tuberculata (Wesmael, 1838, Exothecus) \\ aculeator (Marshall, 1889, Dolops) \\ hastifer (Marshall, 1889, Dolops) \\ caucasica (Tobias, 1976, Doryctodes) \\ rhodopea (Zaykov, 1980, Rhyssalus)
}

Distribution: England, Scotland, Ireland

Notes: some distribution data from Shaw (1993) 


\section{Genus Oncophanes Förster, 1863}

Nomenclature:

EPIRHYSSALUS Ashmead, 1900

\section{Oncophanes minutus (Wesmael, 1838)}

\section{Nomenclature:}

Exothecus minutus Wesmael, 1838

lanceolator (Nees, 1834, Bracon) preocc.

laevigatus (Ratzeburg, 1852, Bracon)

Distribution: England, Wales, Scotland

Notes: Traditionally treated as a species separate from minutus (e.g. Huddleston 1978;

Fauna Europaea), laevigatus was synonymised by Belokobylskij (1998); we follow this, although it seems likely that there are two species involved.

Genus Pseudobathystomus Belokobylskij, 1986

\section{Pseudobathystomus funestus (Haliday, 1836)}

Nomenclature:

Rogas funestus Haliday, 1836

schmiedeknechti (Fahringer, 1930, Bathystomus)

Distribution: England, Scotland

Notes: Listed as a species of Rhysipolis in Huddleston (1978).

\section{Pseudobathystomus tobiasi (Zaykov, 1980)}

Nomenclature:

Oncophanes tobiasi Zaykov, 1980

Distribution: England, Scotland

Notes: NMS, det. Shaw \& van Achterberg, added on Fauna Europaea

\section{Pseudobathystomus vernalis Belokobylskij, 1994}

Distribution: England, Scotland

Notes: NMS, det. Shaw \& van Achterberg, added on Fauna Europaea 
Genus Rhyssalus Haliday, 1833

Nomenclature:

EURHOPTROCENTRUS Tobias, 1977

\section{Rhyssalus clavator Haliday, 1833}

Distribution: England, Scotland, Ireland, Isle of Man

Rhyssalus longicaudis (Tobias \& Belokobylskij, 1991)

Nomenclature:

Eurhoptrocentrus longicaudis Tobias \& Belokobylskij, 1991

Distribution: Wales

Notes: NMS, det. Shaw \& van Achterberg, added on Fauna Europaea

\section{Rogadinae}

\section{Subfamily Rogadinae Förster, 1863}

Notes: Distribution data mostly from NMS and BMNH.

Tribe Aleiodini Muesebeck, 1928

Notes: Resurrected by Zaldívar-Riverón et al. (2008b).

\section{Genus Aleiodes Wesmael, 1838}

Nomenclature:

ROGAS misident.

RHOGAS misident.

PETALODES Wesmael, 1838

NEORHOGAS Szépligeti, 1906

CHELONORHOGAS Enderlein, 1912

Notes: Aleiodes species are further subdivided in Taxapad (Yu et al. 2012) into the subgenera Aleiodes, Chelonorhogas, Neorhogas and Heterogamus (for those occurring in Britain); the latter is considered here to be a distinct genus (see note under Heterogamus). 
species of Aleiodes excluded from the British and Irish list:

[arcticus (Thomson, 1892, Rogas)] MRS has seen the specimens that this record is based upon (in Ipswich Museum) and they are not arcticus.

\section{Aleiodes albitibia (Herrich-Schäffer, 1838)}

Nomenclature:

Rogas albitibia Herrich-Schäffer, 1838

heterogaster Wesmael, 1838

Distribution: England, Scotland, Wales, Ireland

\section{Aleiodes alternator (Nees, 1834)}

\section{Nomenclature:}

Rogas alternator Nees, 1834

geniculator misident.

balteatus (Curtis, 1834, Rogas)

Distribution: England, Scotland, Wales, Ireland

\section{Aleiodes apicalis (Brullé, 1832)}

Nomenclature:

Bracon apicalis Brullé, 1832

ductor misident., in part

reticulator (Nees, 1834, Rogas)

Distribution: England

Notes: to be added by van Achterberg \& Shaw (in prep.); just one British specimen of this Mediterranean species, considered to be an erratic. Synonymy anticipates van Achterberg \& Shaw (in prep.).

\section{Aleiodes apiculatus (Fahringer, 1932)}

Nomenclature:

Rhogas apiculatus Fahringer, 1932

Distribution: England

Notes: to be added by van Achterberg \& Shaw (in prep.) 
Aleiodes assimilis (Nees, 1812)

Nomenclature:

Bracon assimilis Nees, 1812

bicolor misident., in part

zygaenae (Nees, 1834, Rogas)

Distribution: England, Scotland, Ireland

Notes: added by Schwarz and Shaw (2000)

\section{Aleiodes aterrimus (Ratzeburg, 1852)}

Nomenclature:

Bracon aterrimus Ratzeburg, 1852

grandis Giraud, 1857

Distribution: England

Aleiodes bicolor (Spinola, 1808)

Nomenclature:

Bracon bicolor Spinola, 1808

Distribution: England, Scotland, Wales, Ireland

\section{Aleiodes cantherius (Lyle, 1919)}

Nomenclature:

Rhogas cantherius Lyle, 1919

Distribution: England

\section{Aleiodes circumscriptus (Nees, 1834)}

Nomenclature:

Rogas circumscriptus Nees, 1834

Distribution: England, Scotland, Wales, Ireland

Notes: Sensu neotype (to be published by van Achterberg \& Shaw, in prep.). 
Aleiodes compressor (Herrich-Schäffer, 1838)

Nomenclature:

Rogas compressor Herrich-Schäffer, 1838

unicolor (Wesmael, 1838, Petalodes) preocc.

Distribution: England, Scotland, Wales

Aleiodes coxalis (Spinola, 1808)

Nomenclature:

Rogas coxalis Spinola, 1808

ater (Curtis, 1834, Rogas)

tristis Wesmael, 1838

Distribution: England, Scotland, Isle of Man

Notes: Anticipates synonymy to be published by van Achterberg \& Shaw (in prep.).

Aleiodes crassipes (Thomson, 1892)

Nomenclature:

Rogas crassipes Thomson, 1892

Distribution: Wales

Notes: to be added by van Achterberg \& Shaw (in prep.)

Aleiodes cruentus (Nees, 1834)

Nomenclature:

Rogas cruentus Nees, 1834

Distribution: England

Aleiodes dissector (Nees, 1834)

Nomenclature:

Rogas dissector Nees, 1834

aestivalis (Vollenhoven, 1858, Phylax)

Distribution: England, Scotland 
Aleiodes fortipes (Reinhard, 1863)

Nomenclature:

Rogas fortipes Reinhard, 1863

freyi (Hellén, 1927, Rhogas)

Distribution: England

Notes: to be added by van Achterberg \& Shaw (in prep.)

Aleiodes gastritor (Thunberg, 1824)

Nomenclature:

Ichneumon gastritor Thunberg, 1824

circumscriptus misident., in part

testaceus misident., in part

Notes: Distribution data are not given as this name covers an aggregate of at least four species in Britain (van Achterberg \& Shaw, in prep.). Further, it is not clear to which the name gastritor should be applied. Bracon testaceus Spinola, 1808 is actually Rogas luteus, and 'Aleiodes testaceus (Spinola)' is not a valid taxon, although it has been applied to several small, orangeish Aleiodes, epecially in the gastritor aggregate.

\section{Aleiodes grassator (Thunberg, 1824)}

Nomenclature:

Ichneumon grassator Thunberg, 1824

carbonarius Giraud, 1857

flavipalpis (Thomson, 1892, Rogas)

Distribution: England, Scotland

Notes: Anticipates synonymy to be published by van Achterberg \& Shaw (in prep.).

\section{Aleiodes heterostigma (Stelfox, 1953)}

Nomenclature:

Rogas heterostigma Stelfox, 1953

Distribution: Wales, Ireland

\section{Aleiodes hirtus (Thomson, 1892)}

Nomenclature:

Rogas hirtus Thomson, 1892 
Distribution: England, Scotland

Notes: To be added by van Achterberg \& Shaw (in prep.); treated as a synonym of pallidicornis by Belokobylskij et al. (2003), following Papp (1985).

\section{Aleiodes modestus (Reinhard, 1863)}

Nomenclature:

Rogas modestus Reinhard, 1863

piceus (Fahringer, 1932, Rhogas)

Distribution: England, Scotland, Wales

\section{Aleiodes nigriceps Wesmael, 1838}

Distribution: England, Scotland, Wales, Ireland

Notes: to be added by van Achterberg \& Shaw (in prep.); listed as a subspecies of circumscriptus in Taxapad (Yu et al., 2012), following Papp (1999c) and other authors.

\section{Aleiodes nigricornis Wesmael, 1838}

Distribution: England, Scotland, Wales, Ireland

\section{Aleiodes nobilis (Haliday, 1834)}

Nomenclature:

Rogas nobilis Haliday, 1834

ductor misident., in part

medianus (Thomson, 1892, Rogas)

Distribution: England, Scotland, Ireland

Notes: Brought out of synonymy with ductor by Achterberg (1997).

Aleiodes pallidator (Thunberg, 1824)

Nomenclature:

Ichneumon pallidator Thunberg, 1824

ochraceus (Curtis, 1834, Rogas)

unicolor Wesmael, 1838

?pellucens (Telenga, 1941, Rhogas)

Distribution: England 
Notes: some distribution data from Shaw (1977), Shaw (1981)

Aleiodes pallidicornis (Herrich-Schäffer, 1838)

Nomenclature:

Rogas pallidicornis Herrich-Schäffer, 1838

ductor misident., in part

Distribution: Scotland

Notes: to be added by van Achterberg \& Shaw (in prep.); specimen in BMNH (seen by MRS).

Aleiodes pictus (Herrich-Schäffer, 1838)

Nomenclature:

Rogas pictus Herrich-Schäffer, 1838

borealis misident., in part

circumscriptus misident., in part

nigriceps misident., in part

Distribution: England, Scotland, Wales, Ireland

Notes: to be added by van Achterberg \& Shaw (in prep.)

Aleiodes praetor (Reinhard, 1863)

Nomenclature:

Rogas praetor Reinhard, 1863

luteus (Szépligeti, 1906, Neorhogas)

Distribution: England

Aleiodes pulchripes Wesmael, 1838

Distribution: England, Ireland, Isle of Man

Notes: some distribution data from Shaw (1979)

Aleiodes punctipes (Thomson, 1892)

Nomenclature:

Rogas punctipes Thomson, 1892

Distribution: Scotland, Wales, Ireland 


\section{Aleiodes ruficornis (Herrich-Schäffer, 1838)}

Nomenclature:

Rogas ruficornis Herrich-Schäffer, 1838

dimidiatus misident.

gasterator misident.

Distribution: England, Ireland

Aleiodes rugulosus (Nees, 1811)

Nomenclature:

Bracon rugulosus Nees, 1811

pictus (Kokujev, 1898, Rhogas) preocc.

Distribution: England, Scotland, Wales, Ireland

Aleiodes seriatus (Herrich-Schäffer, 1838)

Nomenclature:

Rogas seriatus Herrich-Schäffer, 1838

vittiger Wesmael, 1838

kuslitzkyi (Tobias, 1976, Rogas)

Distribution: England, Ireland

Aleiodes signatus (Nees, 1812)

Nomenclature:

Bracon signatus Nees, 1812

geniculator (Nees, 1834, Rogas)

annulipes (Herrich-Schäffer, 1838, Rogas)

esseni Hellén, 1927

Distribution: England, Wales

Aleiodes similis (Curtis, 1834)

Nomenclature:

Rogas similis Curtis, 1834

circumscriptus misident., in part 
testaceus misident., in part

spathuliformis (Curtis, 1834, Rogas)

subucola (Curtis, 1834, Rogas)

armatus Wesmael, 1838

Distribution: England, Scotland, Ireland

Notes: The name armatus has been misapplied to a range of undescribed, mediumsized, orange species, at least three of which occur in Britain and Ireland and will be described by van Achterberg \& Shaw (in prep.).

\section{Aleiodes testaceus (Telenga, 1941)}

\section{Nomenclature:}

Heterogamus testaceus Telenga, 1941

Distribution: England, Wales

Notes: to be added by van Achterberg \& Shaw (in prep.)

\section{Aleiodes ungularis (Thomson, 1892)}

Nomenclature:

Rogas ungularis Thomson, 1892

Distribution: England, Wales, Ireland

Notes: To be added by van Achterberg \& Shaw (in prep.); English and Welsh records from specimens seen by MRS in, respectively, the Hope Department, Oxford, and Doncaster Museum.

\section{Aleiodes unipunctator (Thunberg, 1824)}

Nomenclature:

Ichneumon unipunctator Thunberg, 1824

ductor (Thunberg, 1824, Ichneumon)

irregularis Wesmael, 1838

Distribution: England, Scotland, Wales, Ireland

Notes: Anticipates synonymy to be published by van Achterberg \& Shaw (in prep.). 


\section{Aleiodes sp. $L$ van Achterberg \& Shaw, in prep.}

Nomenclature:

borealis misident.

circumscriptus misident., in part

nigriceps misident., in part

Distribution: England, Scotland, Wales, Ireland, Isle of Man

Notes: To be added by van Achterberg \& Shaw (in prep.); this species, whose name is not yet published (van Achterberg \& Shaw, in prep.), is very common in the British Isles and M.R. Shaw det. labels (as borealis) have been left in several British collections (up to 2007). The true $A$. borealis (Thomson, 1892, Rogas) has not been found in the British Isles.

\section{Genus Heterogamus Wesmael, 1838}

Notes: Usually treated as a subgenus of Aleiodes (as in Taxapad: Yu et al. 2012) but Zaldivar-Riverón et al. (Zaldívar-Riverón et al. 2004, Zaldívar-Riverón et al. 2008b) have shown that, on the basis of venom apparatus characters and molecular sequence data, respectively, Heterogamus species form a clade distinct from Aleiodes species. Murray (1939) and Shaw (2000a) published some distribution records.

\section{Heterogamus dispar (Haliday, 1833)}

Nomenclature:

Rogas dispar Haliday, 1833

dispar (Curtis, 1834, Rogas) preocc.

crypticornis (Wesmael, 1838, Aleiodes)

Distribution: England, Scotland, Wales, Ireland

\section{Heterogamus excavatus Telenga, 1941}

\section{Nomenclature:}

farmakena Maláč, 1941

Distribution: England

Notes: Added by Shaw (2000a); Irish records (O'Connor et al. 1999) are erroneous (Shaw 2000a). 
Tribe Clinocentrini van Achterberg, 1991

\title{
Genus Clinocentrus Haliday, 1833
}

Nomenclature:

\author{
CAMPTOCENTRUS Kriechbaumer, 1894 \\ MICRORHOGAS Cameron, 1910 \\ NEORHYSSALUS Baker, 1917
}

Notes: Nomenclature and distribution data from Belokobylskij (1995) and NMS.

species of Clinocentrus excluded from the British and Irish list:

[tenuicornis (Thomson, 1891, Exothecus)] A species of uncertain status (Belokobylskij, pers. comm. to MRS), listed as a synonym of excubitor in Taxapad (Yu et al. 2012), following Szépligeti (1906).

\section{Clinocentrus brevicalcar (Thomson, 1891)}

Nomenclature:

Exothecus brevicalcar Thomson, 1891

Distribution: England, Scotland

\section{Clinocentrus cunctator (Haliday, 1836)}

Nomenclature:

Rogas cunctator Haliday, 1836

analis (Wesmael, 1838, Exothecus)

gracilipes (Thomson, 1892, Exothecus)

Distribution: England, Scotland, Wales, Ireland

Clinocentrus excubitor (Haliday, 1836)

Nomenclature:

Rogas excubitor Haliday, 1836

marginellus (Wesmael, 1838, Exothecus)

Distribution: England, Scotland, Wales, Ireland 
Clinocentrus exsertor (Nees, 1811)

Nomenclature:

Bracon exsertor Nees, 1811

orbitator (Nees, 1834, Bracon)

striolatus (Thomson, 1891, Exothecus)

tarsalis Ashmead, 1894

Distribution: England, Scotland

Clinocentrus hungaricus Szépligeti, 1906

Distribution: England, Scotland

Notes: NMS, BMNH, det. Shaw, added here

Clinocentrus umbratilis Haliday, 1833

Nomenclature:

petiolaris (Thomson, 1891, Exothecus)

polonicus Fahringer, 1931

Distribution: England, Scotland, Ireland

\section{Clinocentrus vestigator (Haliday, 1836)}

Nomenclature:

Rogas vestigator Haliday, 1836

stigmaticus Marshall, 1897

jaroshevskyi Telenga, 1941

obsoletus (Hellén, 1957, Oncophanes)

Distribution: England, Scotland, Ireland, Isle of Man

Tribe Rogadini Förster, 1863

Nomenclature:

PELECYSTOMINI Viereck, 1918

\section{Genus Rogas Nees, 1818}

Nomenclature:

PELECYSTOMA Wesmael, 1838 
RHOGAS Agassiz, 1846

Rogas luteus Nees, 1834

Nomenclature:

testaceus (Fabricius, 1798, Ichneumon) preocc.

testaceator (Thunberg, 1824, Ichneumon)

\section{Genus Triraphis Ruthe, 1855}

Notes: species of Triraphis excluded from the British and Irish list:

[tricolor (Wesmael, 1838, Pelecystoma); syn. solitarius (Watanabe, 1970, Pelecystoma); synonymy by Papp (1995a)] Listed by Huddleston (1978), probably on the basis of the host-parasitoid catalogue of Morley and Rait-Smith (1933), but this publication included non-British rearings from Lepidoptera that occur in Britain and there is no evidence that tricolor, a parasitoid of Apoda limacodes (Hufnagel) (Lepidoptera: Limacodidae), has ever been found in Britain or Ireland.

\section{Sigalphinae}

Subfamily Sigalphinae Haliday, 1833

Tribe Acampsini van Achterberg \& Austin, 1992

Genus Acampsis Wesmael, 1835

Acampsis alternipes (Nees, 1816)

Nomenclature:

Sigalphus alternipes Nees, 1816

Distribution: England

Tribe Sigalphini Haliday, 1833

Genus Sigalphus Latreille, 1802

Nomenclature:

SPHAEROPYX Illiger, 1807

RHITIGASTER Wesmael, 1835

RHYTIDOGASTER Agassiz, 1846 
Notes: species of Sigalphus excluded from the British and Irish list:

[irrorator (Fabricius, 1775, Ichneumon); syn. niger (Retzius, 1783, Ichneumon); syn. globulifer (Geoffroy, 1785, Ichneumon); irroratrix (Schulz, 1906, Sphaeropyx)] Shaw and Huddleston (1991) were unable to trace any British specimens.

\section{Acknowledgements}

Kees van Achterberg kindly answered many braconid queries and provided much advice on the classification of Opiinae. Natalie Dale-Skey did a lot of valuable technical work on getting the data from text files into import spreadsheets. We are very grateful to the photographers who have let us use their lovely images.

\section{Author contributions}

The sections on subfamilies Microgastrinae and Rogadinae are mainly the work of MRS, the section on Dacnusini (Alysiinae) is mainly the work of HCJG, who also contributed to the Alysiini and Opiinae sections. The rest of the work was a joint effort between GRB and MRS, with the editing done by GRB.

\section{References}

- $\quad$ Achterberg Cv (1975) About the identity of Biosteres (Biosteres) impressus (Wesmael) (Braconidae, Opiinae). Entomologische Berichten 35: 175-176.

- Achterberg Cv (1979) A revision of the subfamily Zelinaeauct. (Hymenoptera, Braconidae). Tijdschrift voor Entomologie 122: 241-479.

- $\quad$ Achterberg Cv (1984a) Addition to the revision of the genus Zele Curtis (Hymenoptera: Braconidae). Entomologische Berichten 44: 110-112.

- $\quad$ Achterberg Cv (1984b) Essay on the phylogeny of Braconidae (Hymenoptera: Ichneumonoidea). Entomologisk Tidskrift 105: 41-58.

- Achterberg Cv (1985) The genera and subgenera of Centistini, with description of two new taxa from the Nearctic Region (Hymenoptera: Braconidae: Euphorinae). Zoologische Mededelingen 59: 348-362.

- Achterberg Cv (1987) Revision of the European Helconini (Hymenoptera: Braconidae: Helconinae). Zoologische Mededelingen 61 (18): 263-285.

- Achterberg Cv (1988a) Revision of the subfamily Blacinae Foerster (Hymenoptera, Braconidae). Zoologische Verhandelingen 249: 1-324.

- Achterberg Cv (1988b) The genera of the Aspilota-group and some descriptions of fungicolous Alysiini from the Netherlands (Hymenoptera: Braconidae: Alysiinae). Zoologische Verhandelingen 247: 1-88.

- $\quad$ Achterberg Cv (1989) Revision of the subtribe Monoctonina Mackauer sensu stricto (Hymenoptera: Braconidae: Aphidiinae). Zoologische Mededelingen 63 (1): 1-22. 
- $\quad$ Achterberg Cv (1990) Revision of the Western Palaearctic Phanerotomini (Hymenoptera: Braconidae). Zoologische Verhandeligen 255: 1-106.

- $\quad$ Achterberg Cv (1992a) Revisionary notes on the subfamily Homolobinae (Hymenoptera: Braconidae). Zoologische Mededelingen 66 (25): 359-368.

- Achterberg Cv (1992b) Revision of the European species of the genus Pygostolus Haliday (Hymenoptera: Braconidae: Euphorinae), with a key to the Holarctic species. Zoologische Mededelingen, Leiden 66 (24): 349-358.

- $\quad$ Achterberg Cv (1993a) Illustrated key to the subfamilies of the Braconidae (Hymenoptera: Ichneumonoidea). Zoologische Verhandeligen 283: 1-189.

- Achterberg Cv (1993b) Revision of the subfamily Macrocentrinae Foerster (Hymenoptera: Braconidae) from the Palaearctic region. Zoologische Verhandelingen 286: 1-110.

- Achterberg Cv (1995) New combinations of names for Palaearctic Braconidae (Hymenoptera). Zoologische Mededelingen, Leiden 69 (12): 131-138.

- Achterberg Cv (1997) Revision of the Haliday collection of Braconidae (Hymenoptera). Zoologische Verhandeligen 314: 1-115.

- $\quad$ Achterberg Cv (1998) Phaenocarpa helophilae spec. nov. (Hymenoptera: Braconidae), a gregarious parasitoid of Helophilus larvae (Diptera: Syrphidae), with notes on related species. Zoologische Mededelingen 72: 15-27.

- $\quad$ Achterberg Cv (2000) The European species of the Eubazus aliochinoi-group (Hymenoptera: Braconidae: Helconinae: Brachistini). Zoologische Mededelingen, Leiden 74 (20): 339-357.

- $\quad$ Achterberg Cv (2003a) The European species of the subgenus Aliolus Say of the genus Eubazus Nees and of the genus Dicyrtaspis van Achterberg (Hymenoptera: Braconidae: Brachistinae). Zoologische Mededelingen 77 (17): 301-320.

- Achterberg Cv (2003b) Western Palaearctic genera of the subfamily Microgastrinae: a re-appraisal of the generic and tribal division (Hymenoptera: Braconidae). In: Melika G, Thuróczy C (Eds) Parasitic wasps: Evolution, Systematics, Biodiversity and Biological Control [2002]. Agroinform, Budapest, Hungary, 16 pp.

- $\quad$ Achterberg Cv (2003c) The West Palaearctic species of the genera Gildoria Hedqvist and Platyspathius Viereck, with keys to the species (Hymenoptera: Braconidae: Doryctinae). Zoologische Mededelingen 77 (15): 267-290.

- $\quad$ Achterberg Cv (2004a) New Indo-Australian subgenera and species of the genera Xynobius Foerster and Ademoneuron Fischer (Hymenoptera: Braconidae: Opiinae). Zoologische Mededelingen 78 (20): 313-329.

- $\quad$ Achterberg Cv (2004b) Bitomoides gen. nov. (Hymenoptera: Braconidae: Opiinae) from Europe. Zoologische Mededelingen 78 (21): 331-335.

- $\quad$ Achterberg Cv (2011) Order Hymenoptera, family Braconidae. The subfamily Agathidinae from the United Arab Emirates, with a review of the fauna of the Arabian Peninsula. Arthropod fauna of the UAE 4: 286-352.

- $\quad$ Achterberg Cv (2014) Notes on the checklist of Braconidae (Hymenoptera) from Switzerland. Mitteilungen der Schweizerischen Entomologischen Gesellschaft 87: 191-213.

- $\quad$ Achterberg Cv, Broad GR (2013) Revision of the genus Vadumasonium Kammerer (Hymenoptera, Braconidae, Brachistinae). Journal of Hymenoptera Research 33: 91-98. DOI: $10.3897 / j h r .33 .5399$ 
- $\quad$ Achterberg Cv, Haeselbarth E (1983) Revisionary notes on the European species of Macrocentrus Curtis sensu stricto (Hymenoptera: Braconidae). Entomofauna 4: 37-59.

- $\quad$ Achterberg Cv, Haeselbarth E (2003) Revision of the genus Syntretus Foerster (Hymenoptera: Braconidae: Euphorinae) from Europe. Zoologische Mededelingen 77 (2): 9-78.

- Achterberg Cv, Long KD (2010) Revision of the Agathidinae (Hymenoptera, Braconidae) of Vietnam, with the description of forty-two new species and three new genera. ZooKeys 54: 1-184. DOI: 10.3897/zookeys.54.475

- Achterberg Cv, O'Connor JP (1990) Revision of the Palaearctic genus Trachyusa Ruthe (Hymenoptera: Braconidae). Zoologische Mededelingen 64 (8): 107-112.

- $\quad$ Achterberg Cv, Polaszek A (1996) The parasites of cereal stem borers (Lepidoptera: Cossidae, Crambidae, Noctuidae, Pyralidae) in Africa, belonging to the family Braconidae (Hymenoptera: Ichneumonoidae). Zoologische Verhandeligen 304: 1-123.

- Achterberg Cv, Salvo A (1997) Reared Opiinae (Hymenoptera: Braconidae) from Argentina. Zoologische Mededelingen 71: 189-214.

- $\quad$ Achterberg Cv, Shaw MR (2008) A new subgenus of the genus Colastes Haliday (Hymenoptera: Braconidae: Exothecinae) for species reared from bracket fungi, with description of two new species from Europe. Journal of Natural History 42: 1849-1860. DOI: $10.1080 / 00222930802114280$

- $\quad$ Achterberg Cv, Vikberg V (2014) Dinotrema brevis spec. nov. (Hymenoptera: Braconidae: Alysiinae), a new brachypterous species from Finland. Zoologische Mededelingen 88 (1): 1-7.

- $\quad$ Allen AA (1978) The discovery of Apanteles gades Nixon (Hymenoptera: Braconidae) in Britain. Entomologist's Gazette 29: 102.

- Baker EA (2013) Aphid parasitoids in Wales. British Journal of Entomology and Natural History 26: 219-239.

- $\quad$ Baker EA, Broad GR (2009) Five aphid parasitoids (Hymenoptera: Braconidae: Aphidiinae) new to Britain. British Journal of Entomology and Natural History 22: 255-263.

- Baker EA, Broad GR (2013) Eight aphid parasitoids (Hymenoptera: Braconidae: Aphidiinae) new to Britain. British Journal of Entomology and Natural History 26: 187-197.

- $\quad$ Belokobylskij SA (1986) [A review of the Palaearctic species of the genera Pambolus Hal. and Dimerus Ruthe (Hymenoptera, Braconidae).] (in Russian). Trudy Zoologicheskogo Instituta. Leningrad 159: 18-37.

- $\quad$ Belokobylskij SA (1992) [On the classification and phylogeny of braconid wasps subfamilies Doryctinae and Exothecinae (Hymenoptera, Braconidae). I. Classification. Entomological Review 72: 109-137.] (in Russian). Entomologicheskoe Obozrenie 71: 900-928.

- $\quad$ Belokobylskij SA (1995) Revision of the European species of the genus Clinocentrus (Hymenoptera, Braconidae). Journal of Natural History 29: 803-836. DOI: 10.1080/0022 2939500770261

- $\quad$ Belokobylskij SA (1998) 1. Rhyssalinae, 2. Doryctinae, 3. Histeromerinae, 4. Exothecinae, 7. Gnamptodontinae, 9. Alysiinae (Alysiini), 10. Helconinae, 11. Cenocoeliinae, 12. Brachistinae, 14. Meteorideinae, 16. Xiphozelinae, 17. Homolobinae, 18. Charmontinae, 19. Orgilinae, 20. Ecnomiinae, 21. Sigalphinae, 23. Ichneutinae, 25. Cardiochilinae, 27. Dirrhopinae, 28. Miracinae, 29. Adeliinae. In: Ler 
PA (Ed.) Key to the insects of Russian Far East. Vol. 4. Neuropteroidea, Mecoptera, Hymenoptera. Pt 3. Dal'nauka, Vladivostok, $121 \mathrm{pp}$.

- $\quad$ Belokobylskij SA (2000a) Braconidae. In: Ler PA (Ed.) [Key to the insects of Russian Far East. Vol. IV. Neuropteroidea, Mecoptera, Hymenoptera. Pt 4.] Opredelitel nasekomykh Dalnego Vostoka Rossii. T. IV. Dalnauka, Vladivostok, 651 pp. pp.

- Belokobylskij SA (2000b) On the Asian species of the genus Streblocera Westwood, 1833 (Hymenoptera: Braconidae, Euphorinae) with a key to the eastern Palaearctic species (continuation). Entomologische Zeitschrift 110: 290-297.

- Belokobylskij SA, Samartsev KG (2014) Palaearctic species of the Spathius exarator species group (Hymenoptera: Braconidae: Doryctinae) with entirely sculptured mesopleuron. Zootaxa 3900 (4): 483-504. DOI: 10.11646/zootaxa.3900.4.2

- Belokobylskij SA, Tobias VI (1986) Doryctinae. In: Medvedev GS (Ed.) Opredelitel Nasekomych Evrospeiskoi Tsasti SSSR 3, Peredpontdatokrylye 4. Opr. Faune SSSR. 145: 1-501. $51 \mathrm{pp}$.

- Belokobylskij SA, Zaldivar-Riverón A, Quicke DL (2004) Phylogeny of the genera of the parasitic wasps subfamily Doryctinae (Hymenoptera: Braconidae) based on morphological evidence. Zoological Journal of the Linnean Society 142 (3): 369-404. DOI: 10.1111/j.1096-3642.2004.00133.x

- $\quad$ Belokobylskij SA, Taeger A, van Achterberg C, Haeselbarth E, Riedel M (2003) Checklist of the Braconidae of Germany (Hymenoptera). Beiträge zur Entomologie 53 (2): 341-435.

- $\quad$ Belshaw R, Quicke DL (1997) A molecular phylogeny of the Aphidiinae (Hymenoptera: Braconidae). Molecular Phylogenetics and Evolution 7 (3): 281-293. DOI: 10.1006/ mpev.1996.0400

- Belshaw R, Quicke DL (2002) Robustness of ancestral state estimates: evolution of life history strategy in ichneumonoid parasitoids. Systematic Biology 51 (3): 450-477. DOI: 10.1080/10635150290069896

- Belshaw R, Dowton M, Quicke DL, Austin AD (2000) Estimating ancestral geographical distributions: a Gondwanan origin for aphid parasitoids? Proceedings of the Royal Society of London series B, Biology 267: 491-496.

- Belshaw R, Lopez-Vaamonde C, Degerli N, Quicke DL (2001) Paraphyletic taxa and taxonomic chaining: evaluating the classification of braconine wasps (Hymenoptera:

Braconidae) using 28S D2-3 rDNA sequences and morphological characters. Biological Journal of the Linnean Society 73 (4): 411-424. DOI: 10.1111/

j.1095-8312.2001.tb01370.x

- $\quad$ Broad GR (2009) Syntretusbreviradialis van Achterberg \& Haeselbarth (Hymenoptera: Braconidae), new to Britain. British Journal of Entomology and Natural History 22: 253.

- Broad GR (2014) Checklist of British and Irish Hymenoptera - Introduction. Biodiversity Data Journal 2: e1113. DOI: 10.3897/BDJ.2.e1113

- $\quad$ Broad GR, Livermore L (2014a) Checklist of British and Irish Hymenoptera - Evanioidea . Biodiversity Data Journal 2: e1116. DOI: 10.3897/BDJ.2.e1116

- $\quad$ Broad GR, Livermore L (2014b) Checklist of British and Irish Hymenoptera Ceraphronoidea . Biodiversity Data Journal 2: e1167. DOI: 10.3897/BDJ.2.e1167

- $\quad$ Capek M, Achterberg Cv (1992) A revision of the genus Microtypus Ratzeburg (Hymenoptera: Braconidae). Zoologische Mededelingen 66 (21): 323-338. 
- Chen X, Achterberg Cv (1997) Revision of the subfamily Euphorinae (excluding the tribe Meteorini Cresson) (Hymenoptera: Braconidae) from China. Zoologische Verhandeligen 313: 1-217.

- Curtis J (1837) A guide to an arrangement of British insects; being a catalogue of all the named species hitherto discovered in Great Britain and Ireland. Second edition, greatly enlarged. 2nd. J. Pigot, London, $294 \mathrm{pp}$.

- $\quad$ Disney RH, Munk T (2005) The scuttle fly host Megaselia giraudii (Egger) (Diptera: Phoridae) and the first British records of its parasitoid Dinotrema necrophilum (Hedqvist, 1972) (Hymenoptera: Braconidae). Entomologist's Gazette 56: 105-106.

- Dowton M, Belshaw R, Austin AD, Quicke DL (2002) Simultaneous molecular and morphological analysis of braconid relationships (Insecta: Hymenoptera: Braconidae) indicates independent mt-tRNA gene inversions within a single wasp family. Journal of Molecular Evolution 54: 210-226. DOI: 10.1007/s00239-001-0003-3

- $\quad$ Eady RD, Clark JA (1964) A revision of the genus Macrocentrus Curtis (Hym., Braconidae) in Europe, with descriptions of four new species. Entomologist's Gazette 15: 97-127.

- $\quad$ Elliot EA, Morley C (1911) On the hymenopterous parasites of Coleoptera. First Supplement. Transactions of the Entomological Societyof London 1911: 452-496.

- Enobakhare DA (2001) Description and a key to Pauesia spp. (Hymenoptera: Aphidiidae) attacking conifer associated lachnids (Homoptera: Aphidoidea: Lachnidae) in Britain. Applied Tropical Agriculture 6 (2): 98-106.

- Faure JC (1924) Observations biologiques sur Bracon glaphyrus Marsh. Revue de Pathologie Vegetale et d'Entomologie Agricole de France 11: 70-72.

- Fernández-Triana J (2015) A revision of the genus Protomicroplitis Ashmead (Hymenoptera, Braconidae, Microgastrinae), with the description of a new species. Zootaxa 4039: 529-542. DOI: http://dx.doi.org/10.11646/zootaxa.4039.4.3

- $\quad$ Fischer M (1958) Die europäischen Arten der Gattung Opius Wesm. Teil 1b (Hymenoptera, Braconidae). Annali del Museo Civico di Storia Naturale di Genova 70: 245-304.

- $\quad$ Fischer M (1960) Die europäischen Arten der Gattung Opius Wesmael, Teil IVa. Annales Zoologici. Warszawa 19: 33-112.

- $\quad$ Fischer M (1967) Über gezüchtete Opiinae aus Europa (Hymenoptera, Braconidae). Zeitschrift für Angewandte Entomologie 60: 318-350. DOI: 10.1111/ j.1439-0418.1967.tb02076.x

- $\quad$ Fischer M (1972) HymenopteraBraconidae (Opiinae I). (Paläarktische Region). Das Tierreich 91(1973): 1-620.

- $\quad$ Fischer M (1980) Fünf neue Raupenwespen (Hymenoptera, Braconidae). Frustula Entomologica 1[1978]: 147-160.

- $\quad$ Fischer M (1997) Die paläarktischen Opiinae (Madenwespen) der Zoologischen Staatssammlung München (Hymenoptera, Braconidae). Entomofauna 18 (14): 137-196.

- $\quad$ Fischer M (2005) Some new Opiinae (Insecta: Hymenoptera: Braconidae) in the Natural History Museum Vienna. Annalen des Naturhistorischen Museums in Wien 106B: 107-133.

- Fischer M (2006) New species of Opiinae (Insecta: Hymenoptera: Braconidae) in the collection of the National Museums of Scotland, Edinburgh. Annalen des Naturhistorischen Museums in Wien 107B: 131-144. 
- $\quad$ Fischer M, Koponen M (1999) A survey of Opiinae (Hymenoptera, Braconidae) of Finland, part 1. Entomologica Fennica 10: 65-93.

- Fischer M, Tormos J, Docavo I, Pardo X (2004) A new species of Antrusa and three new species of Chorebus (Hymenoptera: Braconidae) from the Iberian Peninsula. Florida Entomologist 87: 306-311. DOI: 10.1653/0015-4040(2004)087 [0306:ansoaa]2.0.co;2

- $\quad$ Fitton MG, Graham MWdV, Boucek ZR, Fergusson ND, Huddleston T, Quinlan J, Richards OW (1978) Kloet and Hincks. A check list of British insects. Part 4: Hymenoptera . Handbooks for the Identification of British Insects 11: ix + 159 pp.

- Fulmek L (1968) Parasitinsekten der Insektengallen Europas. Beiträge zur Entomologie 18: 719-952.

- Gärdenfors U (1986) Taxonomic and biological revision of Palearctic Ephedrus Haliday (Hymenoptera: Braconidae, Aphidiinae). Entomologica Scandinavica Supplement 27: 1-95.

- $\quad$ Gebiola M, Garonna AP, Bernardo U, Belokobylskij SA (2015) Molecular phylogenetic analyses and morphological variation point to taxonomic problems among four genera of parasitoid doryctine wasps (Hymenoptera : Braconidae). Invertebrate Systematics 29: 591-609. DOI: $10.1071 /$ is 14064

- Godfray HC (1984) Intraspecific variation in the leaf-miner parasite Exotela cyclogaster Forster (Hymenoptera: Braconidae). Proceedings and Transactions of the British Entomological and Natural History Society 17: 47-50.

- Godfray HC (1986) Four species of Opius (Hym., Braconidae) new to Britain. Entomologist's Monthly Magazine 122: 127.

- $\quad$ Godfray HC (1988) Biosteres spinaciae (Thomson) (Hym., Braconidae, Opiinae), new to Britain. Entomologist's Monthly Magazine 124: 251-252.

- $\quad$ Godfray HC, Achterberg Cv (2015) Seven species of parasitoid wasp (Hymenoptera: Braconidae, Alysiinae \& Opiinae) new to the British Isles. Entomologist's Monthly Magazine 151: 273-276.

- Godfray HC, Bland KP (2011) Three species of parasitoid wasp (Braconidae, Alysiinae) new to Britain. British Journal of Entomology and Natural History 24: 127-131.

- Godfray HC, McGavin GC (1985) Colastes pubicornis (Thomson) (Hym., Braconidae, Exothecini) new to Britain, with a first host record. Entomologist's Monthly Magazine 121: $109-110$.

- Griffiths GC (1964) The Alysiinae (Hym. Braconidae) parasites of the Agromyzidae (Diptera). I. General questions of taxonomy, biology and evolution. Beiträge zur Entomologie 14: 823-914.

- Griffiths GC (1967a) The Alysiinae (Hym. Braconidae) parasites of the Agromyzidae (Diptera) IV. The parasites of Hexomyza Enderlein, Melanagromyza Hendel, Ophiomyia Braschnikov and Napomyza Westwood. Beiträge zur Entomologie 17: 653-696.

- Griffiths GC (1967b) The Alysiinae (Hym. Braconidae) parasites of the Agromyzidae (Diptera) III. The parasites of Paraphytomyza Enderlein, Phytagromyza Hendel, and Phytomyza Fallén. Beiträge zur Entomologie 16(1966): 775-951.

- Griffiths GC (1967c) The Alysiinae (Hym. Braconidae) parasites of the Agromyzidae (Diptera) II. The parasites of Agromyza Fallén. Beiträge zur Entomologie 16(1966): 551-605. 
- Griffiths GC (1968a) The Alysiinae (Hym. Braconidae) parasites of the Agromyzidae (Diptera) V. The parasites of Liriomyza Mik and certain small genera of Phytomyzinae . Beiträge zur Entomologie 18: 5-62.

- $\quad$ Griffiths GC (1968b) The Alysiinae (Hym. Braconidae) parasites of the Agromyzidae (Diptera) VI. The parasites of Cerodontha Rondani s.I. Beiträge zur Entomologie 18: 63-152.

- Griffiths GC (1984) The Alysiinae (Hym. Braconidae) parasites of the Agromyzidae (Diptera). VII. Supplement. Beiträge zur Entomologie 34: 343-362.

- Haeselbarth E (1973) Die Blacus-Arten Europas und Zentral-Asiens. Veröffentlichungen der Zoologischen Staatssammlung München 16: 69-170.

- Haeselbarth E (1988) Zur Braconidengattung Townesilitus Haeselbarth \& Loan, 1983. Entomofauna 9: 429-460.

- Haeselbarth E (1996) Rilipertus gen. nov., eine neue Gattung der Euphorinae (Hymenoptera, Braconidae). Entomofauna 17: 397-412.

- Haeselbarth E (1999) Zur Braconiden-Gattung Perilitus Nees, 1818 2. Beitrag: Die Arten mit ausgebildetem ersten Cubitus-Abschnitt. Mitteilungen der Münchner Entomologischen Gesellschaft 89: 11-46.

- Haeselbarth E (2008) Zur Braconiden-Gattung Perilitus Nees 1818 3. Beitrag: Die Arten ohne ausgebildetem ersten Cubitus-Abschnitt (Hymenoptera, Braconidae) [On the braconid genus Perilitus Nees 1818 - 3rd contribution: The species with the first segment of the cubitus extinguished (Hymenoptera, Braconidae]. Linzer Biologische Beiträge 40 (2): 1013-1152.

- Huddleston T (1978) Braconidae and Aphidiidae . In: Fitton MG, Graham MWdV, Bouček ZR, Fergusson ND, Huddleston T, Quinlan J, Richards OW (Eds) Kloet and Hincks. A check list of British insects. Part 4: Hymenoptera. Handbooks for the Identification of British Insects 11: ix + 159 pp., 16 pp.

- Huddleston T (1980) A revision of the western Palaearctic species of the genus Meteorus (Hymenoptera: Braconidae). Bulletin of the British Museum (Natural History) (Entomology) 41 (1): 1-58.

- Huddleston T (1984) The Palearctic species of Ascogaster (Hymenoptera: Braconidae). Bulletin of the British Museum (Natural History), Entomology 49 (5): 341-392.

- Hussey NW (1952) A Braconid (Hym.) parasite of Ochina ptinoides Marsh. (Col., Anobiidae). Entomologist's Monthly Magazine 88: 201.

- $\quad$ ICZN (1988) Opinion 1510. Microgaster Latreille, 1804 (Insecta, Hymenoptera): Microgaster australis Thomson, 1895 designated as the type species. Bulletin of Zoological Nomenclature 45: 239-240.

- Jennings MT (2008) A record of Baryproctus barypus (Marshall) (Hym., Braconidae) as a parasitoid of Lipara rufitarsis Loew (Dipt., Chloropidae) from Kent, U.K. Entomologist's Monthly Magazine 144: 192.

- Jennings MT (2012) Bracon leptus Marshall, 1897 (Hymenoptera:Braconidae) reared in Britain. Entomologist's Monthly Magazine 148: 54.

- Kavallieratos NG, Tomanovic Z, Stary P, Athanassiou CG, Fasseas C, Petrovic O, Stanisavljevic LZ, Veroniki MA (2005) Praon Haliday (Hymenoptera: Braconidae: Aphidiinae) of southeastern Europe: key, host range and phylogenetic relationships. Zoologischer Anzeiger 243 (3): 181-209. DOI: 10.1016/j.jcz.2004.11.001

- Kerrich GJ (1932) Additions to the Ichneumonoid fauna of Wicken Fen. Natural History of Wicken Fen. 6. Cambridge, 6 pp. 
- Kloet GS, Hincks WD (1945) A check list of British insects. Stockport by the Authors, Stockport, $483 \mathrm{pp}$.

- König R (1972) Zur Systematik, Faunistik, Phänologie und Ökologie mitteleuropäischer Braconiden (Hymenoptera) (1). Faunistisch-Ökologische Mitteilungen 4: 85-106.

- Kotenko AG, Tobias VI (1986) Subfamily Microgastrinae . In: Tobias VI (Ed.) Keys to the Insects of the European part of the USSR 3. Hymenoptera. Part 4. Leningrad [In Russian. English translation 1995, pp. 605-816. Science Publishers.], 344-459 pp.

- Kula RR (2008) Taxonomic status and location of type specimens for species of Coelinidea Viereck and Sarops Nixon (Hymenoptera: Braconidae: Alysiinae) described by Garland T. Riegel. Journal of Hymenoptera Research 17: 138-156.

- Laing F (1928) A note on two parasites of Lyctus parallelopipedus Mel. Entomologist 61: 163-164.

- $\quad$ Liston AD, Knight GT, Sheppard DA, Broad GR, Livermore L (2014) Checklist of British and Irish Hymenoptera - Sawflies, 'Symphyta'. Biodiversity Data Journal 2: e1168. DOI: 10.3897/BDJ.2.e1168

- $\quad$ Li X-, Achterberg Cv, Tan J- (2013) Revision of the subfamily Opiinae (Hymenoptera, Braconidae) from Hunan (China), including thirty-six new species and two new genera. ZooKeys 268: 1-186. DOI: 10.3897/zookeys.326.5911

- Loan CC (1974) The European species of Leiophron Nees and Peristenus Foerster (Hymenoptera : Braconidae, Euphorinae). Transactions of the Royal Entomological Society of London 126 (2): 207-238. DOI: 10.1111/j.1365-2311.1974.tb00852.x

- Loan CC (1976) Peristenus malatus (Hymenoptera: Braconidae, Euphorinae) a new species, parasitic on Blepharidopterus angulatus (Fallén) (Heteroptera: Miridae) in Britain. Le Naturaliste Canadien 103: 437-440.

- $\quad$ Luff ML (1976a) Centistes nasutus (Wesm.) (Hym., Braconidae) parasitising Amara apricaria Payk. (Col., Carabidae). Entomologist's Monthly Magazine 112: 40.

- Luff ML (1976b) The biology of Microctonus caudatus (Thomson) a braconid parasite of the ground beetle Harpalus rufipes (Degeer). Ecological Entomology 1: 111-116. DOI: 1 0.1111/j.1365-2311.1976.tb01211.x

- Lyle GT (1933) A catalogue of the British Braconidae. Transactions of the Royal Entomological Society of London 81 (1): 67-74. DOI: 10.1111/ j.1365-2311.1933.tb00399.x

- Mackauer M (1961) Die Typen der Unterfamilie Aphidiinae des Britischen Museums London (Hymenoptera: Braconidae). Beiträge zur Entomologie 11: 96-154.

- Mancini D, Priore R, Battaglia D, Achterberg Cv (2003) Caenopachys hartigii (Ratzeburg) (Hymenoptera: Braconidae: Doryctinae) confirmed for Italy, with notes on the status of the genus Caenopachys Foerster. Zoologische Mededelingen 77 (26): 459-470.

- Marshall TA (1872) A catalogue of British Hymenoptera; Chrysididae, Ichneumonidae, Braconidae, and Evanidae. The Entomological Society of London, London, $136 \mathrm{pp}$.

- Marshall TA (1885) Monograph of British Braconidae. Part I. Transactions of the Entomological Society of London 1885: 1-280. DOI: $\underline{10.1111 /}$ j.1365-2311.1885.tb00886.x

- Marshall TA (1887) Monograph of British Braconidae. Part II. Transactions of the Entomological Society of London 1887: 51-131. DOI: $\underline{10.1111 /}$ j.1365-2311.1887.tb00920.x 
- $\quad$ Marshall TA (1896) Les Braconides. In: André E (Ed.) Species des Hymenopteres d'Europe et d'Algerie. Tome 5, 635 pp.. Gray 1891

- Marshall TA (1900) Les Braconides (Supplément). In: André E (Ed.) Species des Hymenopteres d'Europe et d'Algerie. Species des Hymenopteres d'Europe et d'Algerie. Paris.

- Mason WRM (1981) The polyphyletic nature of Apanteles Foerster (Hymenoptera, Braconidae) - a phylogeny and reclassification of Microgastrinae. Memoirs of the Entomological Society of Canada 115: 1-147. DOI: 10.4039/entm113115fv

- Morley C (1913) On Walker's Japanese Ichneumonidae . Entomologist 46: 131-135.

- Morley C, Rait-Smith W (1933) The Hymenopterous parasites of the British Lepidoptera . Transactions of the Royal Entomological Society of London 81: 133-183.

- Müller CB, Adriaanse IC, Belshaw R, Godfray HC (1999) The structure of an aphidparasitoid community. Journal of Animal Ecology 68: 346-370. DOI: 10.1046/ j.1365-2656.1999.00288.x

- $\quad$ Munk T, Peris-Felipo JF, Jiménez-Peydró R (2013) New western Palaearctic Dinotrema species with mesoscutal pit and only medially sculptured propodeum (Hymenoptera, Braconidae, Alysiinae). ZooKeys 260: 61-76. DOI: 10.3897/zookeys.260.4084

- Murray J (1939) Heterogamus dispar Curtis in Dumfriesshire. Entomologist 72: 170.

- New TR (1970) The life histories of two species of Leiophron Nees (Hymenoptera, Braconidae) parasitic on Psocoptera in southern England. Entomologist's Gazette 21: 39-48.

- Nixon GE (1937) British species of Dacnusa (Braconidae). Transactions of the Society for British Entomology 4: 1-88.

- Nixon GE (1943) A revision of the European Dacnusini (Hym., Braconidae, Dacnusinae). Entomologist's Monthly Magazine 79: 20-34.

- Nixon GE (1944) A revision of the European Dacnusini (Hym., Braconidae, Dacnusinae). Entomologist's Monthly Magazine 80: 88-108.

- Nixon GE (1945) A revision of the European Dacnusini (Hym., Braconidae, Dacnusinae). Entomologist's Monthly Magazine 81: 189-204.

- Nixon GE (1946) A revision of the European Dacnusini (Hym., Braconidae, Dacnusinae). Entomologist's Monthly Magazine 82: 279-300.

- Nixon GE (1948) A revision of the European Dacnusini (Hym., Braconidae, Dacnusinae). Entomologist's Monthly Magazine 84: 207-224.

- Nixon GE (1949) A revision of the European Dacnusini (Hym., Braconidae, Dacnusinae). Entomologist's Monthly Magazine 85: 289-298.

- Nixon GE (1954) A revision of the European Dacnusini (Hym., Braconidae, Dacnusinae). Entomologist's Monthly Magazine 90: 257-290.

- Nixon GE (1965) A reclassification of the tribe Microgasterini (Hymenoptera : Braconidae). Bulletin of the British Museum (Natural History) (Entomology) Supplement 2: 1-284.

- $\quad$ Nixon GE (1968) A revision of the genus Microgaster Latreille (Hymenoptera : Braconidae). Bulletin of the British Museum (Natural History), Entomology 22 (2): 31-72. DOI: $10.5962 /$ bhl.part.9950

- Nixon GE (1970) A revision of the N.W. European species of Microplitis Förster (Hymenoptera: Braconidae). Bulletin of the British Museum (Natural History), Entomology 25 (1): 1-30. 
- Nixon GE (1972) A revision of the north-western European species of the laevigatusgroup of Apanteles Förster (Hymenoptera, Braconidae). Bulletin of Entomological Research 61: 701-743. DOI: 10.1017/s0007485300047544

- Nixon GE (1973) A revision of the north-western European species of the vitripennis, pallipes, octonarius, triangulator, fraternus, formosus, parasitellae, metacarpalis and circumscriptus-groups of Apanteles Förster (Hymenoptera, Braconidae). Bulletin of Entomological Research 63: 169-228. DOI: 10.1017/s0007485300039006

- Nixon GE (1974) A revision of the north-western European species of the glomeratusgroup of Apanteles Förster (Hymenoptera, Braconidae). Bulletin of Entomological Research 64: 453-524. DOI: 10.1017/s0007485300031333

- Nixon GE (1976) A revision of the north-western European species of the merula, lacteus, vipio, ultor, ater, butalidis, popularis, carbonarius and validus-groups of Apanteles Förster (Hymenoptera, Braconidae). Bulletin of Entomological Research 65: 687-732. DOI: $10.1017 / \mathrm{s} 0007485300006386$

- Nixon GE (1986) A revision of the European Agathidinae (Hymenoptera: Braconidae). Bulletin of the British Museum (Natural History), Entomology 52 (3): 183-242.

- Notton DG (1991) Aspilota intermediana Fischer a fungicolous braconid (Hym.) new to Britain. Entomologist's Monthly Magazine 127: 159-160.

- $\quad$ Notton DG, Popovici OA, Achterberg Cv, De Rond J, Burn JT (2014) Parasitoid wasps new to Britain (Hymenoptera: Platygastridae, Eurytomidae, Braconidae \& Bethylidae). European Journal of Taxonomy 99: 1-20. DOI: 10.5852/ejt.2014.99

- O'Connor JP, Nash R, Achterberg Cv (1999) A catalogue of the Irish Braconidae (Hymenoptera: Ichneumonoidea). Occasional Publications of the Irish Biogeographical Society 4: 1-90.

- $\quad$ Papp J (1972) New Apanteles Först. species from Hungary (Hymenoptera, Braconidae: Microgasterinae), I. Annales Historico-naturales Musei Nationalis Hungarici 64: 335-345.

- $\quad$ Papp J (1974) Zur Kenntnis der Bracon-Arten Österreichs (Hymenoptera, Braconidae). Annales Historico-Naturales Musei Nationalis Hungarici 78: 415-435.

- $\quad$ Papp J (1976) Key to the European Microgaster Latr. Species, with a new species and taxonomical remarks (Hymenoptera: Braconidae, Microgasterinae). Acta Zoologica Academiae Scientiarum Hungaricae 22: 97-117.

- Papp J (1978) A survey of the European species of Apanteles Först. (Hymenoptera, Braconidae: Microgasterinae), II. The laevigatus-group, 1. Annales Historico-Naturales Musei Nationalis Hungarici 70: 265-301.

- Papp J (1981) A survey of the European species of Apanteles Först. (Hymenoptera, Braconidae: Microgastrinae), V. The lacteus-, longipalpis-, ultor-, butalidis- and vipiogroup. Annales Historico-Naturales Musei Nationalis Hungarici 73: 263-291.

- Papp J (1983) A survey of the European species of Apanteles Först. (Hymenoptera, Braconidae: Microgastrinae), VII. The carbonarius-, circumscriptus-, fraternus-, pallipes -, parasitellae-, vitripennis-, liparidis-, octonarius and thompsoni-group. Annales Historico-naturales Musei Nationalis Hungarici 75: 247-283.

- $\quad$ Papp J (1984) Palaearctic species of Microgaster Latreille (=Microplitis Förster) with description of seven new species (Hymenoptera, Braconidae, Microgastrinae). Entomologische Abhandlungen 47: 95-140.

- Papp J (1985) Contributions to the Braconid fauna of Hungary, VII. Rogadinae (Hymenoptera: Braconidae). Folia Entomologica Hungarica 46: 143-164. 
- $\quad$ Papp J (1987) A survey of the European species of Apanteles Först. (Hymenoptera, Braconidae: Microgastrinae), X. The glomeratus-group 2 and the cultellatus-group. Annales Historico-Naturales Musei Nationalis Hungarici 79: 207-258.

- Papp J (1988) A survey of the European species of Apanteles Först. (Hymenoptera, Braconidae: Microgastrinae) XI. "Homologization" of the species-groups of Apanteless. I. with Mason's generic taxa. Checklist of genera. Parasitoid / host list 1. Annales Historico-Naturales Musei Nationalis Hungarici 80: 145-175.

- $\quad$ Papp J (1992) Bracon strobilorum Ratzeburg and its related species (Hymenoptera: Braconidae, Braconinae). Folia Entomologica Hungarica 53: 129-134.

- Papp J (1993) New Braconid wasps (Hymenoptera, Braconidae) in the Hungarian Natural History Museum, 4. Annales Historico-Naturales Musei Nationalis Hungarici 84: 155-180.

- $\quad$ Papp J (1995a) Braconidae (Hymenoptera) from Korea, XVII. Annales HistoricoNaturales Musei Nationalis Hungarici 87: 123-127.

- $\quad$ Papp J (1995b) Revision of C. Wesmael's Chelonus species (HymenopteraBraconidaeCheloninae). Bulletin de l'Institut Royal des Sciences Naturelles de Belgique, Entomologie 65: 115-134.

- Papp J (1996a) The braconid wasps of (Hymenoptera, Braconidae) of the Bükk National Park (NE Hungary). The Fauna of the Bükk National Park 2: 453-476.

- $\quad$ Papp J (1996b) Taxonomic revision of C. Rondani's braconid species (HymenopteraBraconidae). Redia 78[1995] (2): 303-309.

- $\quad$ Papp J (1996c) Contribution to the braconid fauna of Hungary, XI. Cheloninae and Sigalphinae (Hymenoptera: Braconidae). Folia Entomologica Hungarica 57: 131-156.

- Papp J (1997) Taxonomic revision of seven European species of the genus Bracon Fabricius (Hymenoptera: Braconidae). Folia Entomologica Hungarica 58: 115-135.

- Papp J (1998) Contributions to the braconid fauna of Cyprus (Hymenoptera, Braconidae: Braconinae). Entomofauna 19: 241-251.

- Papp J (1999a) A revision of the Bracon species described by O. Schmiedeknecht (Insecta: Hymenoptera: Braconidae: Braconinae). Entomologische Abhandlungen 58: 289-308.

- $\quad$ Papp J (1999b) The braconid wasps (Hymenoptera: Braconidae) of the Aggtelek National Park (N.E. Hungary). In: Mahunka S (Ed.) The Fauna of the Aggtelek National Park. Vol. II. Hungarian Natural History Museum, Budapest, 25 pp.

- Papp J (1999c) Two new species of Bracon from Britain (Hym., Braconidae, Braconinae). Entomologist's Monthly Magazine 135: 145-152.

- Papp J (2000) First synopsis of the species of obscurator species-group, genus Bracon, subgenus Glabrobracon (Hymenoptera: Braconidae, Braconinae). Annales HistoricoNaturales Musei Nationalis Hungarici 92: 229-264.

- $\quad$ Papp J (2002) The Braconid wasps (Hymenoptera: Braconidae) of the Ferto-Hansag National Park (NW Hungary). The Fauna of the Fertö-Hansag National Park. 24 pp.

- $\quad$ Papp J (2003) Type specimens of the braconid species described by T. A. Marshall and deposited in the Hungarian Natural History Museum (Hymenoptera: Braconidae). Annales Historico-Naturales Musei Nationalis Hungarici 95: 135-146.

- Papp J (2004a) Type specimens of the braconid species by Gy. Szépligeti deposited in the Hungarian Natural History Museum (Hymenoptera: Braconidae). Annales HistoricoNaturales Musei Nationalis Hungarici 96: 153-223. 
- $\quad$ Papp J (2004b) A Bakony-hegység gyilkosfürkész faunájának alapvetése (Hymenoptera, Braconidae) V. Agathidinae, Alysiinae [A monograph of the braconid fauna of the Bakony Mountains (Hymenoptera, Braconidae) V. Agathidinae, Alysiinae]. Folia Musei Historico-Naturalis Bakonyiensis 21: 111-154.

- Papp J (2004c) A revision of Szépligeti's Microchelonus species described from Hungary (Hymenoptera: Braconidae: Cheloninae). Annales Historico-Naturales Musei Nationalis Hungarici 96: 225-259.

- $\quad$ Papp J (2005a) A revision of the Bracon (Lucobracon) species described by Szépligeti from the Western Palaearctic Region (Hymenoptera: Braconidae: Braconinae). Annales Historico-Naturales Musei Nationalis Hungarici 97: 197-224.

- $\quad$ Papp J (2005b) A checklist of the Braconidae of Hungary (Hymenoptera). Folia Entomologica Hungarica 66: 137-194.

- $\quad$ Papp J (2007) Braconidae (Hymenoptera) from Korea XXII. Subfamily Alysiinae . Acta Zoologica Academiae Scientiarum Hungaricae 53 (1): 1-38.

- Papp J (2008a) A revision of the Bracon (subgenera Bracon s.str., Cyanopterobracon, Glabrobracon, Lucobracon, Osculobracon subgen. n., Pigeria) species described by Szépligeti from the western Palaearctic Region (Hymenoptera: Braconidae, Braconinae). Linzer Biologische Beiträge 40 (1): 1741-1837.

- Papp J (2008b) Redescriptions of Habrobracon concolorans (Marshall) and Habrobracon crassicornis (Thomson) (Hymenoptera: Braconidae: Braconinae). Entomologisk Tidskrift 129 (3): 165-172.

- $\quad$ Papp J (2009a) Braconidae (Hymenoptera) from Mongolia, XVII. Eleven subfamilies. Acta Zoologica Academiae Scientiarum Hungaricae 55: 139-173.

- Papp J (2009b) Contribution to the braconid fauna of the former Yugoslavia, V. Ten subfamilies. Entomofauna 30 (1): 1-36.

- Papp J (2012) A revision of the Bracon Fabricius species in Wesmael's collection deposited in Brussels (Hymenoptera: Braconidae: Braconinae). European Journal of Taxonomy 21: 1-154. DOI: http://dx.doi.org/10.5852/ejt.2012.21

- Pennacchio F (1989) The Italian species of the genus Aphidius Nees (Hymenoptera, Braconidae, Aphidiinae). Bollettino del Laboratorio di Entomologia Agraria Filippo Silvestri 46: 75-106.

- Perepechayenko VL (2000) [Review of genera of the tribe Dacnusini (Hymenoptera: Braconidae: Alysiinae) of Palaearctic region] (in Russian). Izvestiya Kharkovskogo Entomologitchestogo Obshchestva 8: 57-79.

- Perkins JF (1953) Notes on the British Ichneumoninae with descriptions of new species (Hym., Ichneumonidae). Bulletin of the British Museum (Natural History) (Entomology) 3 (4): 9-176.

- Perkins JF, Nixon GE (1939) Insecta, Hymenoptera, Ichneumonoidea . In: Salzman F (Ed.) The Victoria history of the counties of England: A history of the county of Oxford. Vol. 1. Oxford University Press, London, 5 pp.

- $\quad$ Powell W (1982) The identification of hymenopterous parasitoids attacking cereal aphids in Britain. Systematic Entomology 7: 465-473. DOI: $10.1111 /$ j.1365-3113.1982.tb00457.x

- $\quad$ Pungerl NB (1986) Morphometric and electrophoretic study of Aphidius species (Hymenoptera: Aphidiidae) reared from a variety of aphid hosts. Systematic Entomology 11: 327-354. DOI: 10.1111/j.1365-3113.1986.tb00185.x 
- $\quad$ Quicke DL, Sharkey MJ (1989) A key to and notes on the genera of Braconinae (Hymenoptera: Braconidae) from America north of Mexico with descriptions of two new genera and three new species. Canadian Entomologist 121: 337-361. DOI: 10.4039/

\section{Ent121337-4}

- Rakhshani E, Tomanovic Z, Stary P, Kavallieratos NG, Ilic M, Stankovic SS, RajabiMazhar N (2011) Aphidiinae parasitoids (Hymenoptera: Braconidae) of Macrosiphoniella aphids (Hemiptera: Aphididae) in the western Palaearctic region. Journal of Natural History 45: 2559-2575. DOI: 10.1080/00222933.2011.597004

- Rakhshani E, Stary P, Hidalgo NP, Čkrić J, Moghaddam MG, Tomanovic S, Petrovic A, Tomanovic Z (2015) Revision of the world Monoctonia Starý, parasitoids of gall aphids: taxonomy, distribution, host range, and phylogeny (Hymenoptera, Braconidae: Aphidiinae). Zootaxa 3905 (4): 474-488. DOI: 10.11646/zootaxa.3905.4.2

- Revels R (2006) More on the rise and fall of the Holly Blue. British Wildlife 17 (6): 419-424.

- $\quad$ Richards OW (1960) On some British species of Perilitus Nees (Hymenoptera: Braconidae). Proceedings of the Royal Entomological Society of London (B) 29: 140-144. DOI: 10.1111/j.1365-3113.1960.tb01160.x

- $\quad$ Richards OW (1967) Some British species of Leiophron Nees (Hymenoptera: Braconidae), with the description of two new species. Transactions of the Royal Entomological Society of London 119 (2): 171-186. DOI: 10.1111/ j.1365-2311.1967.tb00508.x

- Roman A (1912) Die Ichneumonidentypen C.P. Thunbergs. Zoologiska Bidrag fran Uppsala 1: 230-293.

- Schwarz M, Shaw MR (2000) Western Palaearctic Cryptinae (Hymenoptera: Ichneumonidae) in the National Museums of Scotland, with nomenclatural changes, taxonomic notes, rearing records and special reference to the British check list. Part 3. Tribe Phygadeuontini, subtribes Chiroticina, Acrolytina, Hemitelina and Gelina (excluding Gelis), with descriptions of new species. Entomologist's Gazette 51: 147-186.

- $\quad$ Sharanowski BJ, Dowling AP, Sharkey MJ (2011) Molecular phylogenetics of Braconidae (Hymenoptera: Ichneumonoidea), based on multiple nuclear genes, and implications for classification. Systematic Entomology 36: 549-572. DOI: 10.1111/ j.1365-3113.2011.00580.x

- Sharkey MJ, Stoelb SA (2012) Revision of Therophilus s.s. (Hymenoptera, Braconidae, Agathidinae) from Thailand. Journal of Hymenoptera Research 27: 1-36. DOI: $10.3897 /$ jhr.27.2832

- $\quad$ Sharkey MJ, Laurenne NM, Sharanowski B, Quicke DL, Murray D (2006) Revision of the Agathidinae (Hymenoptera: Braconidae) with comparisons of static and dynamic alignments. Cladistics 22: 546-567. DOI: 10.1111/j.1096-0031.2006.00121.x

- Sharkey MJ, Yu DS, van Noort S, Seltmann K, Penev L (2009) Revision of the Oriental genera of Agathidinae (Hymenoptera, Braconidae) with an emphasis on Thailand including interactive keys to genera published in three different formats. ZooKeys 21 : 19-54. DOI: 10.3897/zookeys.21.271

- $\quad$ Shaw MR (1977) Aleiodes pallidator (Thunberg) (=unicolor Wesmael) (Hym., Braconidae) new to Britain. Entomologist's Monthly Magazine 113: 81.

- Shaw MR (1979) Rogas pulchripes (Wesmael) (Hymenoptera: Braconidae) and other parasites of arboreal Acronicta species (Lepidoptera: Noctuidae) at Chat Moss, Manchester. Entomologist's Gazette 30: 291-294. 
- $\quad$ Shaw MR (1981) Undue alarm over parasitism (Hym.) of Clostera anachoreta (D. \& S.). Entomologist's Record and Journal of Variation 93: 115.

- Shaw MR (1983) Aphaereta pallipes (Say) (Hym: Braconidae) new to Britain and Palaearctic region, with remarks on other parasites of Diptera. Entomologist's Monthly Magazine 119: 73-74.

- Shaw MR (1988a) Meteorus brevicauda Thomson (Hymenoptera: Braconidae) reared from larvae of Zeugophora subspinosa (Fabricius) (Coleoptera: Chrysomelidae). Entomologist's Gazette 39: 205-206.

- $\quad$ Shaw MR (1988b) Spathius curvicaudis Ratzeburg (Hym.: Braconidae) new to Britain and parasitising Agrilus pannonicus (Piller and Mitterpacher) (Col.: Buprestidae). Entomologist's Record 100: 215-216.

- $\quad$ Shaw MR (1989) Cosmophorus cembrae Ruschka new to Britain (Hymenoptera: Braconidae: Euphorinae). Entomologist's Gazette 40: 241-243.

- Shaw MR (1992a) Microtypus wesmaelii Ratzeburg (Hymenoptera: Braconidae, Microtypinae), a species and subfamily new to Britain. Entomologist's Gazette 43: 289-291.

- Shaw MR (1992b) A new species of Hygroplitis Thomson in England (Hymenoptera: Braconidae, Microgastrinae). Entomologist's Gazette 43: 283-288.

- $\quad$ Shaw MR (1993) An enigmatic rearing of Dolopsidea indagator (Haliday) (Hymenoptera: Braconidae). The Entomologist's Record and Journal of Variation 105: 31-36.

- Shaw MR (1994) Some recent British specimens of Baryproctus barypus (Marshall) (Hym., Braconidae, Braconinae). Entomologist's Monthly Magazine 130: 219-221.

- $\quad$ Shaw MR (1995) Observations on the adult behaviour and biology of Histeromerus mystacinus Wesmael (Hymenoptera: Braconidae). The Entomologist 114 (1): 1-13.

- $\quad$ Shaw MR (1996a) British records of two species of Blacometeorus Tobias (Hymenoptera: Braconidae, Blacinae). Entomologist's Gazette 47: 267-268.

- $\quad$ Shaw MR (1996b) Chrysopophthorus hungaricus (Zilahi-Kiss) (Hymenoptera: Braconidae, Euphorinae) new to Britain, a parasitoid of adult Chrysopidae (Neuroptera). Entomologist's Gazette 47: 185-187.

- Shaw MR (1997) The genus Heterospilus Haliday in Britain, with descriptions of a new species and remarks on related taxa (Hymenoptera: Braconidae: Doryctinae). Zoologische Mededelingen 71 (5): 33-41.

- Shaw MR (1998) Some genera and species of Doryctinae (Hymenoptera: Braconidae) new to Britain. Entomologist's Gazette 49: 191-194.

- $\quad$ Shaw MR (1999) Rearing records of two species of Cenocoelius Haliday from Britain (Hymenoptera: Braconidae, Cenocoeliinae). Entomologist's Gazette 50: 283-286.

- Shaw MR (2000a) Records of Aleiodes excavatus (Telenga) (Hym., Braconidae) from the British Isles. Entomologist's Monthly Magazine 136: 251.

- Shaw MR (2000b) Two species of Coeloides (Hym., Braconidae, Braconinae) new to Britain, with notes on congeners. Entomologist's Monthly Magazine 136: 137-140.

- Shaw MR (2003a) Adverse comment on the supposed British status of the webspinning sawfly Cephalcia arvensis Panzer (Hymenoptera: Pamphiliidae). British Journal of Entomology and Natural History 16: 2.

- Shaw MR (2003b) Revised synonymy in the genus Cotesia (Hymenoptera: Braconidae: Microgastrinae): the identity of Microgaster vestalis Haliday, 1834, as a senior synonym of Apanteles plutellae Kurdjumov, 1912. Entomologist's Gazette 54: 187-189. 
- Shaw MR (2004) Microgaster alebion Nixon and its 'var A': description of a new species and biological notes (Hymenoptera: Braconidae, Microgastrinae). Entomologist's Gazette 55: 217-224.

- Shaw MR (2005) Rediscovery of Earinustransversus Lyle (Hym.: Braconidae: Agathidinae), a parasitoid of Trichopteryx polycommata (D.\&S.) (Lep.: Geometridae: Larentiinae). Entomologist's Record 117: 85-87.

- Shaw MR (2007) The species of Cotesia Cameron (Hymenoptera: Braconidae: Microgastrinae) parasitizing Lycaenidae (Lepidoptera) in Britain. British Journal of Entomology and Natural History 20: 255-267.

- Shaw MR (2009) Occurrence of the genus Elasmosoma Ruthe (Hymenoptera: Braconidae, Euphorinae) in Britain. British Journal of Entomology and Natural History 22: 49-51.

- Shaw MR (2010) Palaearctic Homolobinae (Hymenoptera: Braconidae) in the National Museums of Scotland, with host and distribution records and a key to British species. Entomologist's Gazette 61: 43-51.

- $\quad$ Shaw MR (2012) Notes on some European Microgastrinae (Hymenoptera: Braconidae) in the National Museums of Scotland, with twenty species new to Britain, new host data, taxonomic changes and remarks, and descriptions of two new species of Microgaster Latreille. Entomologist's Gazette 63: 173-201.

- $\quad$ Shaw MR, Askew RR (1976) Ichneumonoidea (Hymenoptera) parasitic upon leafmining insects of the orders Lepidoptera, Hymenoptera and Coleoptera . Ecological Entomology 1: 127-133. DOI: 10.1111/j.1365-2311.1976.tb01213.x

- Shaw MR, Bailey M (1991) Parasitoids (Hymenoptera: Braconidae, Ichneumonidae, Pteromalidae) and notes on the biology of the fern-boring sawfly Heptamelus ochroleucus (Stephens) (Hymenoptera: Tenthredinidae) in the English Lake District. The Entomologist 110 (3): 103-109.

- $\quad$ Shaw MR, Huddleston T (1991) Classification and biology of braconid wasps (Hymenoptera: Braconidae). Handbooks for the Identification of British Insects 7 (11): 1-126.

- $\quad$ Shaw MR, Jennings MT (2008) Polemochartus melas (Giraud) (Hymenoptera: Braconidae, Alysiinae) new to Britain. Entomologist's Gazette 59: 114-116.

- Shaw MR, Quicke DL (1999) The British genera of Braconinae (Hym., Braconidae). Entomologist's Monthly Magazine 135: 95-101.

- Shaw MR, Sims I (2015) Notes on the biology, morphology, nomenclature and classification of Pseudavga flavicoxa Tobias, 1964 (Hymenoptera, Braconidae, Rhysipolinae), a genus and species new to Britain parasitizing Bucculatrix thoracella (Thunberg) (Lepidoptera, Bucculatricidae). Journal of Hymenoptera Research 42:

21-32. DOI: $10.3897 / J H R .42 .8935$

- Shaw MR, Skelton MJ (2008) Parasitism (Hymenoptera: Braconidae, Microgastrinae) in an apparently adventitious colony of Lymantria dispar (Linnaeus) (Lepidoptera: Lymantriidae) in southern England, with speculations on the biology of Glyptapanteles porthetriae (Muesebeck). Entomologist's Gazette 59: 109-113.

- Shenefelt RD (1973) Braconidae 5. Microgasterinae \& Ichneutinae . Hymenopterorum Catalogus (nova editio) 11: 669-812.

- $\quad$ Shenefelt RD (1974) Braconidae 7. Alysiinae . Hymenopterorum Catalogus (nova editio) 11: $937-1113$. 
- Shenefelt RD (1978) Braconidae 10. Braconinae, Gnathobraconinae, Mesostoinae, Pseudodicrogeniinae, Telengainae, Ypsistocerinae, plus Braconidae in general, major groups, unplaced genera and species. Hymenopterorum Catalogus (nova editio) 15: 1425-1872.

- $\quad$ Simbolotti G, Achterberg Cv (1992) Revision of the West Palaearctic species of the genus Bassus Fabricius (Hymenoptera: Braconidae). Zoologische Verhandeligen 281: $1-80$.

- $\quad$ Simbolotti G, Achterberg Cv (1999) Revision of the West Palaearctic species of the genus Agathis Latreille (Hymenoptera: Braconidae: Agathidinae). Zoologische Verhandeligen 325: 1-167.

- Smith F (1853) List of the specimens of British animals in the collection of the British Museum. Part XIII. Nomenclature of Hymenoptera . Taylor \& Francis, London, 73 pp.

- Starý P (1959) A revision of the genus Dyscritulus Hincks. (Hymenoptera, Braconidae, Aphidiinae). Acta Faunistica Entomologica Musei Nationalis Pragae 5: 69-74.

- $\quad$ Starý P (1973) A review of the Aphidius species (Hymenoptera, Aphidiidae) of Europe. Annotationes Zoologicae et Botanicae, Bratislava 84: 1-85.

- Starý P (1975) Aphidius colemani Viereck: its taxonomy, distribution and host range. Acta Entomologica Bohemoslovaca 72: 156-163.

- Starý P (1978) Parasitoid spectrum of the arboricolous callaphidid aphids in Europe (Hymenoptera, Aphidiidae; Homoptera, Aphidoidea, Callaphididae). Acta entomologica Bohemoslovaka 75: 164-177.

- Stelfox AW, Graham MW (1951a) Notes on the genus Aspilota (Hym., Braconidae, Alysiinae), with descriptions of five new species. The Entomologist's Monthly Magazine 87: 3-7.

- Stelfox AW, Graham MW (1951b) Descriptions of two new species of Aspilota (Hym., Braconidae) from England. Entomologist's Monthly Magazine 87: 222-223.

- Stevens NB, Austin AD, Jennings JT (2010) Synopsis of Australian agathidine wasps (Hymenoptera: Braconidae: Agathidinae). Zootaxa 2480: 1-26.

- Stevens NB, Austin AD, Jennings JT (2011) Diversity, distribution and taxonomy of the Australian agathidine genera Camptothlipsis Enderlein, Lytopylus Foerster and Therophilus Wesmael (Hymenoptera: Braconidae: Agathidinae). Zootaxa 2887: 1-49.

- $\quad$ Stigenberg J, Ronquist F (2011) Revision of the Western Palearctic Meteorini (Hymenoptera, Braconidae), with a molecular characterization of hidden Fennoscandian species diversity. Zootaxa 3084: 1-95.

- $\quad$ Stigenberg J, Shaw MR (2013) Western Palaearctic Meteorinae (Hymenoptera: Braconidae) in the National Museums of Scotland, with rearing, phenological and distributional data, including six species new to Britain, and a discussion of a potential route to speciation. Entomologist's Gazette 64: 251-268.

- $\quad$ Stigenberg J, Boring CA, Ronquist F (2015) Phylogeny of the parasitic wasp subfamily Euphorinae (Braconidae) and evolution of its host preferences. Systematic Entomology 40 (3): 570-591. DOI: 10.1111/syen.12122

- $\quad$ Szépligeti G (1904) Hymenoptera. Fam. Braconidae . Genera Insectorum 22: 1-253.

- Szépligeti G (1906) Braconiden aus der Sammlung des ungarischen NationalMuseums, 1. Annales Historico-Naturales Musei Nationalis Hungarici 4: 547-618.

- $\quad$ Taeger A (1989) Die Orgilus-Arten der Paläarktis (Hymenoptera, Braconidae). Akademie der Landwirtschaftswissenschaften der Deutschen Demokratischen Republik, Berlin, 260 pp. 
- Tobias V, Lozan A (2003) Central-European species of Microchelonus Szépligeti (Hymenoptera, Braconidae) with very big apical aperture of males. Linzer Biologische Beiträge 35 (1): 239-261.

- Tobias VI (1986) [Keys to the Insects of the European Part of the USSR. Volume III Hymenoptera. Part IV.]. Nauka Publisher, Leningrad [English translation published in in 1995, Science Publishers, Inc., New Hampshire, USA], 293-335 pp.

- Tobias VI (1998) Alysiinae (Dacnusini). In: Ler PA (Ed.) [Key to the insects of Russian Far East. Vol. 4. Neuropteroidea, Mecoptera, Hymenoptera. Pt 3.] (in Russian). Dal'nauka. Vladivostok, $112 \mathrm{pp}$.

- $\quad$ Tobias VI, Jakimavicius AB (1973) [Supplementary data about the braconid (Hymenoptera, Braconidae) fauna of Lithuania.] (in Russian with English summary). Acta Entomologica Lituanica 2: 23-28.

- Tobias VI, Shaw MR (2005) Rearing records of two species of Microchelonus Szépligeti from Britain, with the description of a new species (Hym., Braconidae: Cheloninae). Entomologist's Monthly Magazine 141: 15-19.

- Tomanovic Z, Kavallieratos NG, Stary P, Stanisavljevic L, Petrovic-Obradovic S, Tomanovic M, Milutinovic M (2006) Phylogenetic relationships among Praini (Hymenoptera: Braconidae: Aphidiinae) aphid parasitoids, with redescription of two species. Insect Systematics and Evolution 37: 213-226. DOI: 10.1163/18763120678883 1128

- $\quad$ Traugott M, Bell JR, Broad GR, Powell W, Van Veen FJ, Vollhardt IM, Symondson WO (2008) Endoparasitism in cereal aphids: molecular analysis of a whole parasitoid community. Molecular Ecology 17: 3928-3938. DOI: 10.1111/j.1365-294x.2008.03878.x

- $\quad$ Tremblay E, Eady RD (1978) Lysiphlebus confusus n.sp. per Lysiphlebus ambiguus sensu Auct. nec Haliday (1834) (Hymenopteralchneumonoidea). Bollettino del Laboratorio di Entomologia Agraria 'Filippo Silvestri' 35: 180-184.

- Van Veen FJ, Müller CB, Pell JK, Godfray HC (2008) Food web structure of three guilds of natural enemies: predators, parasitoids and pathogens of aphids. Journal of Animal Ecology 77: 191-200. DOI: 10.1111/j.1365-2656.2007.01325.x

- Walker AK, Wharton RA (2011) A review of New World Eurytenes s. str. (Hymenoptera, Braconidae, Opiinae). Journal of Hymenoptera Research 20: 23-46. DOI: $10.3897 /$ jhr.29.877

- Wharton RA (1980) Review of the Nearctic Alysiini (Hymenoptera, Braconidae). With discussion of generic relationships within the tribe. University of California Publications in Entomology 88: 1-112+xi.

- Wharton RA (1983) Variation in Opius hirtus Fischer and discussion of Desmiostoma Foerster (Hymenoptera: Braconidae). Proceedings of the Entomological Society of Washington 85: 327-330.

- Wharton RA (1985) Characterization of the genus Aspilota (Hymenoptera: Braconidae). Systematic Entomology 10: 227-237. DOI: 10.1111/j.1365-3113.1985.tb00528.x

- Wharton RA (1986) The braconid genus Alysia (Hymenoptera): a description of the subgenera and a revision of the subgenus Alysia . Systematic Entomology 11: 453-504. DOI: $10.1111 /$ i. 1365-3113.1986.tb00538.x

- Wharton RA (1988a) The braconid genus Alysia (Hymenoptera): a revision of the subgenus Anarcha . Contributions of the American Entomological Institute 25 (2): 1-69.

- Wharton RA (1988b) Classification of the braconid subfamily Opiinae (Hymenoptera). Canadian Entomologist 120: 333-360. DOI: 10.4039/Ent120333-4 
- Wharton RA (1993) Review of the Hormiini (Hymenoptera: Braconidae) with a description of new taxa. Journal of Natural History 27: 107-171. DOI: 10.1080/00222939 300770061

- Wharton RA (1994) New genera, species, and records of New World Alysiinae (Hymenoptera: Braconidae). Proceedings of the Entomological Society of Washington 96: 630-664.

- Wharton RA (1997) Alysiinae. In: Wharton RA, Marsh PM, Sharkey MJ (Eds) Manual of the New World genera of the family Braconidae (Hymenoptera). International Society of Hymenopterists, Special Publication No. 1, 33 pp.

- Wharton RA (2002) Revision of the Australian Alysiini (Hymenoptera : Braconidae). Invertebrate Systematics 16: 7-105. DOI: 10.1071/IT01012

- Wharton RA (2006) The species of Sternaulopius Fischer (Hymenoptera: Braconidae, Opiinae) and the braconid sternaulus. Journal of Hymenoptera Research 15 (2): 316-347.

- Wharton RA, Austin AD (1991) Revision of Australian Dacnusini (Hymenoptera; Braconidae: Alysiinae): Parasitoids of cyclorrhaphous Diptera . Journal of the Australian Entomological Society 30 (3): 193-206. DOI: 10.1111/j.1440-6055.1991.tb00411.x

- Wilkinson DS (1945) Description of Palaearctic species of Apanteles (Hymen., Braconidae). Transactions of the Entomological Society of London 95: 35-226. DOI: 10. 1111/j.1365-2311.1945.tb00436.x

- $\quad$ Yang Z, Gu Y, Song Y (2003) A new species in the genus Ropalophorus Curtis (Hymenoptera: Braconidae) from China, parasitizing adults of the bark beetle Ips subelongatus (Coleoptera: Scolytidae), with a key to world species of the genus. Zoologische Mededelingen 77 (36): 631-636.

- Yu DS, Achterberg Cv, Horstmann K (2012) Taxapad 2012, Ichneumonoidea 2011. Database on flash-drive. www.taxapad.com,

- Zaldívar-Riverón A, Mori M, Quicke DL (2006) Systematics of the cyclostome subfamilies of braconid parasitic wasps (Hymenoptera: Ichneumonoidea): A simultaneous molecular and morphological Bayesian approach. Molecular Phylogenetics and Evolution 38 (1): 130-145. DOI: 10.1016/j.ympev.2005.08.006

- Zaldívar-Riverón A, Areekul B, Shaw MR, Quicke DL (2004) Comparative morphology of the venom apparatus in the braconid wasp subfamily Rogadinae (Insecta, Hymenoptera, Braconidae) and related taxa. Zoologica Scripta 33 (3): 223-237. DOI: 1 0.1111/j.0300-3256.2004.00144.x

- Zaldívar-Riverón A, Belokobylskij SA, León-Regagnon V, Briceño-G. R, Quicke DL (2008a) Molecular phylogeny and historical biogeography of the cosmopolitan parasitic wasp subfamily Doryctinae (Hymenoptera:Braconidae). Invertebrate Systematics 22: 345-363.

- Zaldívar-Riverón A, Shaw MR, Sáez AG, Mori M, Belokobylskij SA, Shaw SR, Quicke DL (2008b) Evolution of the parasitic wasp subfamily Rogadinae (Braconidae): phylogeny and evolution of lepidopteran host ranges and mummy characteristics. BMC Evolutionary Biology 8 (239): 20 pp.. 


\section{Supplementary materials}

Suppl. material 1: Checklist of British and Irish Hymenoptera

Authors: Broad, G.R., Shaw, M.R. \& Godfray, H.C.J.

Data type: formatted text file

Brief description: Word document version of the checklist

Filename: Hym_checklist_7_Braconidae_March_2016_update.docx - Download file (369.71 kb)

\section{Suppl. material 2: Checklist of British and Irish Braconidae}

Authors: Broad, G.R., Shaw, MR. \& Godfray, H.C.J.

Data type: spreadsheet

Brief description: Excel spreadsheet version of the checklist

Filename: Braconidae checklist spreadsheet March 2016.xlsx - Download file (743.92 kb) 